\title{
Biomolecular engineering in the design of novel therapies to treat coagulation disorders and inflammatory diseases
}

Citation for published version (APA):

Wildhagen, K. C. A. A. (2014). Biomolecular engineering in the design of novel therapies to treat coagulation disorders and inflammatory diseases. [Doctoral Thesis, Maastricht University]. Uitgeverij BOXPress. https://doi.org/10.26481/dis.20141125kw

Document status and date:

Published: 01/01/2014

DOI:

10.26481/dis.20141125kw

Document Version:

Publisher's PDF, also known as Version of record

Please check the document version of this publication:

- A submitted manuscript is the version of the article upon submission and before peer-review. There can be important differences between the submitted version and the official published version of record.

People interested in the research are advised to contact the author for the final version of the publication, or visit the DOI to the publisher's website.

- The final author version and the galley proof are versions of the publication after peer review.

- The final published version features the final layout of the paper including the volume, issue and page numbers.

Link to publication

\footnotetext{
General rights rights.

- You may freely distribute the URL identifying the publication in the public portal. please follow below link for the End User Agreement:

www.umlib.nl/taverne-license

Take down policy

If you believe that this document breaches copyright please contact us at:

repository@maastrichtuniversity.nl

providing details and we will investigate your claim.
}

Copyright and moral rights for the publications made accessible in the public portal are retained by the authors and/or other copyright owners and it is a condition of accessing publications that users recognise and abide by the legal requirements associated with these

- Users may download and print one copy of any publication from the public portal for the purpose of private study or research.

- You may not further distribute the material or use it for any profit-making activity or commercial gain

If the publication is distributed under the terms of Article $25 \mathrm{fa}$ of the Dutch Copyright Act, indicated by the "Taverne" license above, 
Biomolecular engineering in the design of novel therapies to treat coagulation disorders and inflammatory diseases 


\section{Colofon}

(C) Karin Wildhagen, Maastricht 2014

Thesis Maastricht University

ISBN 978-90-8891-997-8

Cover design: Proefschriftmaken.nl || Uitgeverij BOXPress

Printed \& Lay Out by: Proefschriftmaken.nl || Uitgeverij BOXPress

Published by: Uitgeverij BOXPress, 's-Hertogenbosch

All rights reserved. No part of this thesis may be reproduced, stored in a retrieval system or transmitted in any form or by any means, without the permission in writing from the author, or, when appropriate, of the publishers of the publications. 


\title{
Biomolecular engineering in the design of novel therapies to treat coagulation disorders and inflammatory diseases
}

\author{
PROEFSCHRIFT
}

ter verkrijging van de graad van doctor aan de Universiteit Maastricht, op gezag van de Rector Magnificus, Prof. Dr. L.L.G. Soete volgens het besluit van het College van Decanen, in het openbaar te verdedigen op dinsdag 25 november 2014 om 14.00 uur

door

Karin Catharina Adriana Anna Wildhagen Geboren op 3 februari 1985 te Goirle 


\section{Promotores:}

Prof. dr. T. M. Hackeng

Prof. dr. C.P.M. Reutelingsperger

\section{Copromotor:}

Dr. G.A.F. Nicolaes

\section{Beoordelingscommissie:}

Prof. dr. E.A.L. Biessen (voorzitter)

Prof. dr. F.C.S. Ramaekers

Prof. dr. J.J. Voorberg (Sanquin Bloedvoorziening, Amsterdam)

Prof. dr. G. Vriend (Radboud Universiteit, Nijmegen)

Financial support by the Dutch Heart Foundation, Stichting Fonds voor het Hart and Stichting Hartsvrienden RESCAR for the publication of this thesis is gratefully acknowledged 


\section{Contents}

$\begin{array}{lll}\text { Chapter } 1 \quad \text { General Introduction } & 7\end{array}$

Chapter 2 The structure-function relationship of activated protein C: lessons from natural and engineered mutations

Chapter 3 Identification of novel small molecule inhibitors of activated protein $\mathrm{C}$

Chapter 4 Effects of exogenous recombinant APC in mouse models of ischemia reperfusion injury and of atherosclerosis

Chapter 5 Proteolysis of extracellular histones by activated protein C

Chapter 6 Extracellular histone H3 levels are associated with sepsis-induced organ failure and serve as predictive biomarkers of mortality in ICU sepsis patients

Chapter 7 Nonanticoagulant heparin prevents histone-mediated cytotoxicity in vitro and improves survival in sepsis

Chapter 8 Summary and general discussion

Nederlandse Samenvatting

Valorisation

Dankwoord

Curriculum Vitae

List of publications 



\section{Chapter 1}

General Introduction 
In this introductory chapter, first an overview of the coagulation pathways will be given, followed by a detailed description of the anticoagulant protein activated protein $\mathrm{C}$ (APC) and the glycosaminoglycan, heparin. These biomolecules do not only function as anticoagulants, but more interestingly possess several cytoprotective properties. Selective inhibition of the anticoagulant properties of APC or administration of exogenous APC or heparin can, in our opinion, be beneficial in several disease models that are outlined next. Finally, the design of engineered APC and heparin biomolecules with reduced anticoagulant properties is described. We hypothesize that these variants can potentially be used as novel therapies to treat coagulation disorders and inflammatory diseases.

\section{Coagulation}

In vivo coagulation is initiated by exposure of tissue factor (TF) to the blood. TF is expressed at extravascular sites such as adventitial fibroblasts and vascular smooth muscle cells. At sites of vascular injury where the endothelium is disrupted, TF is exposed and factor (F)VII is activated to FVIIa (figure 1). The extrinsic pathway of coagulation is initiated by formation of the extrinsic tenase complex by TF and factor FVIIa (1), that activates the inactive precursor (zymogen) serine protease FX. Alternatively, FX can be activated by the intrinsic tenase complex consisting of FIXa and its cofactor FVIIIa on a surface that contains negatively charged phospholipids. Subsequently FXa forms, together with its cofactor FVa, the prothrombinase complex on a surface of negatively charged phospholipids. This complex converts prothrombin to thrombin, the key-enzyme of the coagulation cascade that mediates conversion of soluble fibrinogen to an insoluble cross-linked fibrin clot.

Thrombin further enhances coagulation via a positive feedback mechanism by activation of the cofactors for the prothrombinase complex (FV) and intrinsic tenase complex (FVIII) and by FXI activation. FXIa on its turn can activate FIX, which is important for FX activation via the intrinsic tenase complex. Furthermore, thrombin facilitates clot stabilization by activation of FXIII, a transglutaminase that cross-links fibrin polymers and thereby supports formation of an insoluble fibrin clot (2-4).

An alternative mechanism by which the coagulation cascade can be initiated is the intrinsic pathway (figure 1). This pathway is initiated by the activation of FXII by binding to a negatively charged surface, such as glass, resulting in conversion of prekallikrein to kallikrein. High molecular weight kininogen (HMWK) is a cofactor for both the activation of FXII and kallikrein. The newly formed kallikrein accelerates conversion of FXII to FXIIa, that subsequently activates FXI $(5,6)$. 
Intrinsic pathway

Surface
Extrinsic pathway

\section{Trauma}

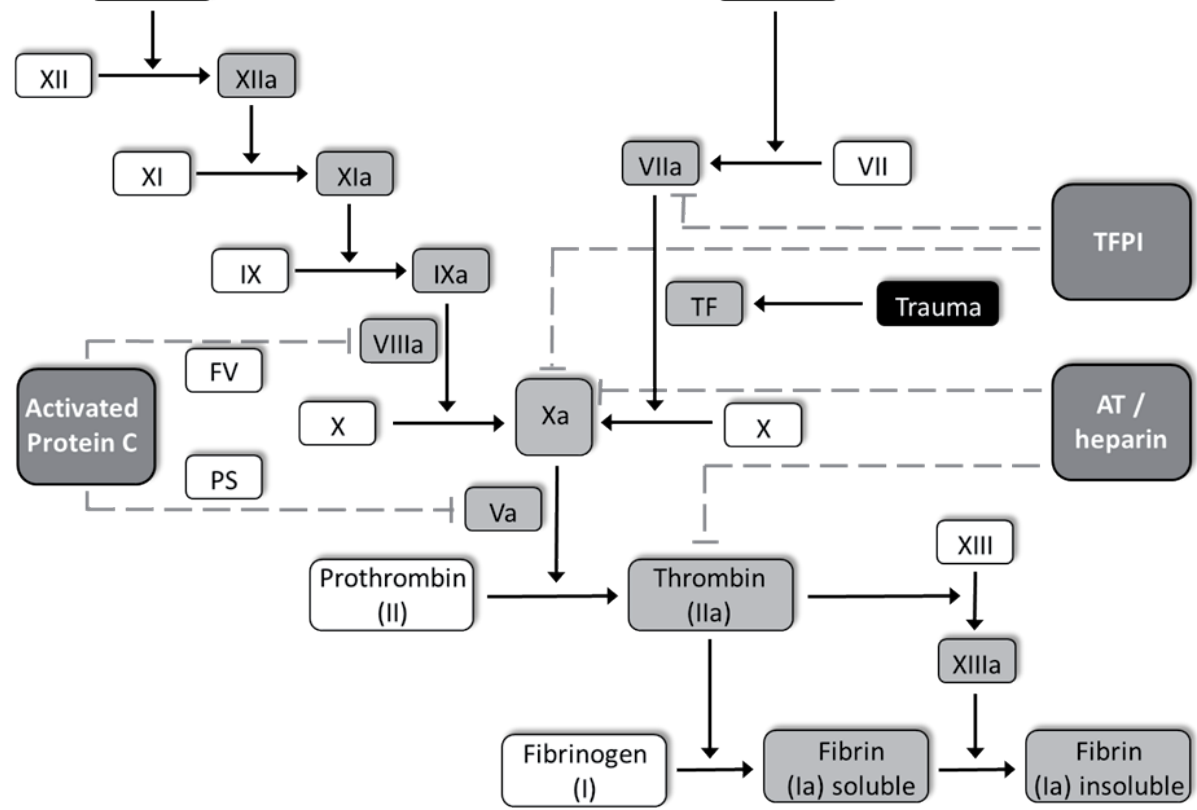

Figure 1: Overview of the coagulation cascade

Both the intrinsic and extrinsic pathway can initiate the common coagulation pathway, resulting in formation of thrombin (IIa) and finally in formation of an insoluble fibrin clot. Activated Protein C, antithrombin (AT) and Tissue factor pathway inhibitor (TFPI) are anticoagulant proteins that inactivate several coagulation proteins. The inactivation of FXa and thrombin by AT is more than 100-fold increased in the presence of heparin. The cofactor activities of protein S (PS) and factor V (FV) are essential for respectively inactivation of FVa and FVIIIa by APC.

\section{Activated protein C}

Protein C (PC) is a vitamin K-dependent glycoprotein of $62 \mathrm{kDa}$ that circulates as an inactive zymogen in human plasma at an average concentration of $65-70 \mathrm{nM}(7,8)$. Thrombin can activate the proteolytically inactive PC by cleavage at arginine (R) 169, resulting in removal of the activation peptide and generation of the serine protease activated PC (APC). In the absence of its cofactors thrombomodulin (TM) and endothelial protein $C$ receptor (EPCR), thrombin is a poor activator of PC (9). However, when thrombin is bound to the endothelium associated transmembrane receptor TM, the activation of PC is accelerated several orders of magnitude (10). Binding of the thrombin-TM complex to EPCR, another receptor that is present on the surface of vascular endothelial cells, enhances its catalytic activity 20-fold (11). The interactions of PC with both EPCR and the thrombin-TM complex are $\mathrm{Ca}^{2+}$-dependent (12).

APC is the central enzyme of the natural anticoagulant protein $C$ pathway that helps to regulate thrombin formation via proteolysis of active coagulation factors $\mathrm{V}(\mathrm{FVa})$ 
and VIII (FVIIIa) (figure 1). The cofactor activities of protein S (PS) and factor V (FV) are essential for respectively inactivation of FVa by proteolysis at R306, R506 and R679 and inactivation of FVIIIa by proteolysis at R336, R562 and R740 (13-15). The crucial role of the APC pathway for maintenance of the haemostatic balance is indicated by the increased risk for thromboembolic events observed in individuals with heterogeneous PC deficiency $(16,17)$ and the life threatening thrombotic complications that occur, even in the presence of aggressive anticoagulant therapy, in individuals with homozygous PC deficiency $(18,19)$.

Initially APC has been identified and characterized as an anticoagulant protein; however recent research (20-25) has shown that APC possesses neuroprotective and cytoprotective functions that likely explain the protective effects of APC in animal models of diseases like ischemic/embolic stroke, myocardial infarction, sepsis and lung injury. These cytoprotective effects of APC are protease activated receptor 1 (PAR-1) and EPCR-dependent and comprise of anti-inflammatory, anti-apoptotic and endothelial barrier stabilizing effects on different cell types $(26,27)$.

\section{Heparin}

Heparin is a highly sulfated anticoagulant polysaccharide belonging to the family of glycosaminoglycans (28). It has the highest negative charge density of all known biological molecules and is biosynthesized and stored in mast cells of particularly the liver, lung and intestinal mucosa (29). All heparin preparations contain polydisperse linear polymers with heterogeneous sequences, so the molecular weight (MW) cannot be uniquely described (30). For unfractionated heparin (UFH) isolated from porcine intestinal mucosa most polymers are in the range from 17 to $20 \mathrm{kDa}$. Low molecular weight heparin (LMWH) preparations have an average MW ranging from 3.6 to 6.5 $\mathrm{kDa}$ (30). Heparin has been used for years as a potent anticoagulant. Binding of heparin to antithrombin changes the conformation of antithrombin and thereby greatly accelerates its inhibitory activity towards mainly serine proteases thrombin and FXa, but also towards FIXa, FXIa and FXIIa (31). Only heparin molecules that contain a specific pentasaccharide motif, including the unusual 3,6 di-O-sulfated 2-N-sulfated glucosamine residue, and that besides this have sufficient chain length (minimum $5 \mathrm{kDa}$ ) to bind both antithrombin and thrombin, can potentiate inhibition of thrombin (30). Therefore, LMWH has drastically reduced anti-thrombin specific activity compared to UFH. 


\section{Disease models studied in this thesis}

\section{Hemophilia $A$}

Hemophilia A is an X chromosome-linked bleeding disorder caused by mutation of the FVIII gene. Usually, affected men have inherited the mutated gene from their mothers that are non-symptomatic carriers, although 30 percent of mutations arise spontaneously without any family history of hemophilia A. The incidence of hemophilia A is estimated at 1 in 5000 male life births (32). Hemophilia A patients can be divided into three categories depending on their FVIII levels. Patients with severe hemophilia A have $<1 \%$ FVIII levels and experience spontaneous bleedings into muscles, joints and soft tissue, predominantly in the absence of a haemostatic challenge like injury, trauma or surgery. Patients with a moderate form of hemophilia A (1-5\% FVIII level) usually show prolonged bleeding after minor trauma or surgery and occasionally pass through spontaneous bleedings. In patients with mild hemophilia A (>5\% FVIII level) spontaneous bleedings are rare, but severe bleeding after major trauma or surgery occurs regularly (33).

For hemophilia A treatment FVIII concentrates are used preferably. Based on the estimated half-life of infused FVIII protein (8-12 hours) and on the pharmacokinetics of FVIII in an individual patient, subsequent intravenous infusions with FVIII concentrates should be scheduled to keep FVIII levels in circulation above a threshold needed to protect patients from bleeding complications. An alternative is continuous infusion, that has the advantage that peaks and troughs in FVIII levels are avoided, but the disadvantage that patients should be monitored frequently for pump failure (33). In spite of optimal medical care and infusions with FVIII concentrates, individuals with severe hemophilia regularly face musculoskeletal complications. Another treatmentrelated complication is the development of neutralizing antibodies to infused FVIII concentrates. This occurs in $20-30 \%$ of patients with severe hemophilia A and $5-10 \%$ of patients with mild or moderate hemophilia $A$ and makes the infusions no longer useful for coagulation (33).

\section{Atherosclerosis}

Atherosclerosis is a progressive chronic inflammatory disease that is characterized by the formation of lesions in the vessel walls of large and medium-sized arteries. Already in children the earliest lesions, called fatty streaks, start to develop. These small lesions are localized just beneath the endothelial layer of the arterial wall and consist of lipidcontaining macrophage-derived foam cells and T lymphocytes (34).

Endothelial dysfunction probably plays an important role in the formation of atherosclerotic lesions. Dysfunctional endothelium secretes vasoactive molecules, cytokines and growth factors, has an increased permeability and upregulated expression 
of cellular adhesion molecules like vascular cell adhesion molecule-1 (VCAM-1), resulting in transmigration of monocytes and $\mathrm{T}$ lymphocytes into the vessel wall $(34,35)$. Risk factors for endothelial dysfunction and thereby the development of atherosclerosis are hypercholesterolemia, hypertension, free radicals, diabetes mellitus, hyperhomocysteinemia and infectious microorganisms such as herpesvirus and Chlamydia pneumonia (36). When these risk factors remain present, the inflammatory process and plaque formation will progress over time by migration and proliferation of smooth muscle cells into the lesion, resulting in intermediate lesions. Macrophages and lymphocytes keep multiplying, become activated and secrete cytokines, chemokines and enzymes that promote cell death and necrotic core formation. Gradually the lesion enlarges and restructures. A fibrous cap is formed over the core of lipid and necrotic tissue and an advanced, complicated lesion arises (36). The thin fibrous cap is prone to rupture, and when rupture occurs, this leads to formation of a thrombus that can occlude the vessel, resulting in ischemia of the heart, brain or extremities $(34,36)$. When these processes, that illustrate the close interplay between vascular wall changes, inflammatory processes and thrombus formation, occur in the heart or brain, they give rise to what is commonly described as cardiovascular infarction or ischemic stroke.

Thus, not only inflammation, but also coagulation is involved in the pathology of atherosclerosis. Extensive bidirectional cross-talk between both systems has been established in atherosclerosis (37). Platelets play an important role in pro-inflammatory conditions. They adhere to both dysfunctional endothelial cells and to circulating immune cells and secrete atherogenic mediators. Numerous coagulation proteins, such as FXa, thrombin and the TF-FVIIa complex are present in atherosclerotic lesions and induce pro-inflammatory signaling via a class of G-Protein coupled receptors called protease activated receptors (PARs). Furthermore, FXIII, fibrinogen and fibrin degradation products were found in progressed atherosclerotic lesions. Notably, heterozygous protein $\mathrm{C}$ deficiency in mice and low levels of APC in clinical studies are associated with a greater extent or severity of atherosclerosis (37).

\section{Myocardial ischemia/ reperfusion injury}

Coronary heart disease (CHD), characterized by plaque formation in coronary arteries, is the main cause of myocardial ischemia/reperfusion (I/R) injury. According to the World Health Organisation (WHO), CHD is the leading cause of death $(12.8 \%$ of all deaths) and disability worldwide (38). When a coronary artery is occluded, the part of the myocardium that is dependent on this artery for its blood supply will become ischemic. This results in several pathological changes in the ischemic part of the myocardium, including increased intracellular calcium and sodium concentrations, mitochondrial membrane depolarization, depletion of ATP, decrease of $\mathrm{pH}$ due to anaerobic respiration, loss of contractile function, production of reactive oxygen species, apoptosis, endothelial dysfunction, platelet aggregation and microembolization. 
The immune system becomes activated, resulting in neutrophil accumulation and cell mediated damage caused by infiltrated macrophages and $\mathrm{T}$ lymphocytes $(38,39)$. Although reperfusion itself also induces cardiomyocyte death, it is important that therapeutic interventions to restore blood flow, like thrombolytic therapy and primary percutaneous coronary intervention, will be started as soon as possible to reduce $\mathrm{I} / \mathrm{R}$ injury and limit the size of myocardial infarction (38).

\section{Sepsis}

Sepsis arises when the body responds to an infection, of either bacteria, fungi or viruses, and the response culminates into a systemic inflammatory response that inflicts damage to own tissues and organs (40). Based on extrapolation of the number of treated sepsis patients in the United States, the estimated worldwide incidence rate of sepsis is 19 million cases per year, (41). According to official criteria (42) sepsis is diagnosed by a proven or suspected clinical infection in combination with at least two systemic inflammatory response syndrome (SIRS) criteria: 1) a body temperature greater than $38^{\circ} \mathrm{C}$ or less than $36{ }^{\circ} \mathrm{C}$; 2) a heart rate greater than 90 beats/minute; 3) tachypnea, manifested by a respiratory rate greater than 20 breaths/minute or hyperventilation, as indicated by a $\mathrm{PaCO}_{2}$ of less than $32 \mathrm{mmHg}$; 4) an alteration in the white blood cell count, such as a count greater than $12.000 / \mathrm{mm}^{3}$, a count less than $4.000 / \mathrm{mm}^{3}$, or the presence of more than $10 \%$ immature neutrophils. Severe sepsis is diagnosed when patients meet the aforementioned criteria and have developed sepsis-induced organ dysfunction, hypoperfusion abnormality (lactic acidosis, oliguria, acute alteration of mental status) or hypotension. Patients are diagnosed with septic shock when they meet the severe sepsis criteria and have hypotension despite adequate fluid resuscitation. Although advances are made in the treatment of sepsis patients by the improved availability of potent antibiotics and the more advanced supportive therapy for failing organs, mortality rates are still $20-30 \%$ for sepsis patients and even higher for severe sepsis and septic shock patients (40).

Increased concentrations of histones and nucleosomes were found in circulation of patients with trauma, systemic inflammation and sepsis $(43,44,45)$. Histones are cationic nuclear proteins that package and order DNA into structural units called nucleosomes. Nucleosomes consist of a superhelix of 146 base pairs DNA wrapped around an octamer containing two copies of histones H2A, H2B, H3 and H4. These extracellular histones and nucleosomes could originate from either excessive apoptotic and necrotic cell death or neutrophil extracellular trap (NET) formation (46-48). NETs are intricate fibrous networks that contain DNA as their major structural component, as well as antimicrobial proteins such as histones and neutrophil elastase. Extracellular traps are not exclusively released by activated neutrophils. Recently it was discovered that other immune cells like macrophages, eosinophils and mast cells can also release extracellular traps to disarm and kill bacteria (48). Extracellular histones possess bactericidal activity 
(49), but unfortunately also exhibit cytotoxic activity towards host cells, including endothelial cells $(50,51)$. So, release of histones into circulation may trigger a positive feedback loop, resulting in more cell death and additional histone release (52).

The mechanism of histone-mediated cytotoxicity is not exactly known yet. One hypothesis is that histones induce channel formation in phospholipid membranes of cells and platelets, resulting in calcium influx, plasma-membrane depolarization and finally cell death (53-55). Another hypothesis is that extracellular histones act as pathogenassociated molecular patterns (PAMPs) through activation of Toll-like receptors (TLR) 2 and TLR4 in vivo, leading to production of cytokines. These cytokines cause severe sterile inflammation and tissue injury and can ultimately lead to development of organ failure and death in mice (56).

\section{Adjustment of the functional properties of APC and heparin to optimize their biological effectiveness}

\section{Activated protein C}

First, we aimed to selectively inhibit the anticoagulant properties of APC with small molecular inhibitors. We believe that this can be beneficial in individual with bleeding tendencies, like hemophilia A patients.

In the murine myocardial ischemia/reperfusion injury and atherosclerosis studies, we did not only investigate the in vivo effects of wild type (wt) APC administration, but we also tested the effect of the so-called S360A-APC variant, firstly described by Gale et al. (57). In this APC variant, the active site serine was mutated to alanine, yielding a protein that lost the ability to proteolyze FVa and FVIIIa, but still retained limited anticoagulant activity through competition with coagulation factors $\mathrm{Xa}$ and IXa for binding to respectively FVa and FVIIIa $(57,58)$. S360A-APC is not able to cleave PAR-1 and is therefore regarded as being devoid of cytoprotective activity (59). However, S360AAPC can still bind to EPCR via its $\gamma$-carboxyglutamic acid (Gla) domain and thereby achieve coupling of PAR-1 to a $\mathrm{G}(\mathrm{i} / \mathrm{o})$ family of G-proteins, resulting in initiation of protective signaling after cleavage of PAR-1 by either APC or thrombin (60).

For the in vitro studies on the proteolysis of histones by APC, we used a second APC variant. In this variant three lysine $(\mathrm{K})$ residues in the 191 loop and two arginine residues in the calcium binding loop of APC were mutated into alanine (KKK191-193AAA and RR229-230AA). Both loops are part of a positively charged exosite on the surface of APC that is important for FVa and FVIIIa binding and cleavage $(61,62)$. Mutation of these 5 residues yields a variant (5A-APC), firstly described by Mosnier et al. (59), with minimal anticoagulant activity that retains normal cytoprotective activity (63). 


\section{Antithrombin affinity depleted heparin}

Antithrombin affinity depleted heparin (AADH) consists of the subfraction of UFH that is not able to bind to a column to which antithrombin was covalently immobilized (64). AADH possesses greatly diminished anticoagulant activity, that is independent of antithrombin-mediated activities and can be attributed to heparin cofactor II stimulation, the plasma concentration of which does not allow complete inhibition of thrombin formation (64).

\section{Outline of the study}

In this study we investigate the diverse properties of the anticoagulant biomolecules APC and heparin both via in vitro and in vivo methods. For some in vivo purposes it is, in our opinion, beneficial to limit the anticoagulant functions of these biomolecules, while preserving their other (cyto)protective properties.

Chapter 2 starts with an extensive overview of the current knowledge on the structure of APC and its dual anticoagulant and cytoprotective functions. Next, the intricate structure-function relationship of APC is elaborately explored using data from various studies that have investigated the effects of different naturally occuring and engineered mutations in APC on the various APC functions, to dissect areas that are important for anticoagulant, cytoprotective and other properties of APC.

When normal thrombin formation is compromised and the haemostatic balance is tipped towards bleeding tendencies, such as in hemophilia A, it would be beneficial to limit the anticoagulant activity of APC. In chapter 3 we use a validated structurebased virtual screening approach to discover small drug-like molecules that inhibit the interaction between a predominant APC exosite and its substrate FVa. We hypothesize that specific inhibition of this substrate-binding exosite in circulating APC, results in promotion of thrombin formation without affecting the cytoprotective properties of APC. The small molecules identified by in silico screening where tested in vitro by functional screening and direct binding analysis.

Due to its anti-inflammatory properties, we hypothesized that APC has the potential to protect against the development of diseases wherein acute or chronic inflammation plays a major role in the pathogenesis. In chapter 4 we investigate the effects of exogenous wt- and S360A-(A)PC administration in murine models of acute myocardial $\mathrm{I} / \mathrm{R}$ injury and the chronic inflammatory disease atherosclerosis. We analyze effects of wt- and S360A-(A)PC on infarct size, atherosclerotic plaque area and phenotype, cytokine levels in circulation and on the innate and adaptive immune system.

In addition to its anticoagulant and cytoprotective properties, it was recently discovered that APC is able to proteolyze histones. In chapter $\mathbf{5}$ we investigate the proteolytic activity of wt-APC and 5A-APC towards histone $\mathrm{H} 3$ and the influence thereon of varying concentrations of calcium and phospholipids. Subsequently, we investigate the 
cytotoxicity of histone $\mathrm{H} 3$ cleavage products towards endothelial cells and identify the cleavage sites for APC in histone $\mathrm{H} 3$.

Increased concentrations of extracellular histones or nucleosomes were found in the circulation of patients with traumatic injury and sepsis. In chapter $\mathbf{6}$ we measured histone $\mathrm{H} 3$ levels in plasma samples of a group of 43 sepsis patients and investigated whether these correlate with disease severity, mortality and parameters reflecting coagulation and tissue homeostasis.

Histones are strongly positively charged proteins and therefore we hypothesize that heparin, a negatively charged polysaccharide, can bind to histones and block their cytotoxicity. The disadvantage of heparin is that it can potentially cause bleeding complications. In chapter 7 we describe the development of a non-coagulant antithrombin affinity depleted heparin (AADH) that may be used to neutralize the cytotoxic properties of histones, without increase of the bleeding tendency. In vitro, we investigated the binding properties of $\mathrm{AADH}$ to free histones and histones incorporated in NETs and the potency of AADH to protect endothelial cells against the histone-induced cytotoxicity. Lastly, we investigated the in vivo efficacy of AADH administration to oppose histone-mediated cytotoxicity and mortality in mouse models of sterile inflammation and sepsis. 


\section{References}

1. Van der Poll $\mathrm{T}$ (2008) Tissue factor as an initiator of coagulation and inflammation in the lung. Crit Care 12 S3.

2. Davie E, Ratnoff O (1964) Waterfall sequence for intrinsic blood clotting. Science 145: 1310-1312.

3. Dahlbäck B (2000) Blood coagulation. Lancet 355: 1627-1632.

4. Macfarlane R (1964) An enzyme cascade in the blood clotting mechanism, and its function as a biochemical amplifier. Nature 202: 498-499.

5. Dahlbäck B, Villoutreix BO (2005) Regulation of blood coagulation by the protein C anticoagulant pathway. Novel insights into structure-function relationships and molecular recognition. Vasc Biol 25: 1311-1320.

6. Riddel JP, Aouizerat BE, Miaskowski C, Lillicrap DP (2007) Theories of blood coagulation. J Pediatr Oncol Nurs 24: 123-131.

7. Gruber A, Griffin JH (1992) Direct detection of activated protein C in blood from human subjects. Blood 79: 2340-2348.

8. Miletich J, Sherman L, Broze G (1987) Absence of Thrombosis in Subjects with Heterozygous Protein C Deficiency. N Engl J Med 317: 991-996.

9. Friedrich U, Pötzsch B, Preissner K, Müller-Berghaus G, Ehrlich H (1994) Calcium-dependent activation of protein $\mathrm{C}$ by thrombin/thrombomudulin: role of negatively charged amino acids within the activation peptide of protein C. Thromb Haemost 72: 567-572.

10. Nicolaes G, Dahlbäck B (2003) Congenital and acquired activated protein C resistance. Semin Vasc Med 3: 33-46.

11. Stearns-Kurosawa D, Kurosawa S, Mollica J, Ferrell G, Esmon C (1996) The endothelial cell protein C receptor augments protein $\mathrm{C}$ activation by the thrombin-thrombomodulin complex. PNAS 96: 1021210216.

12. Fukudome K, Esmon C (1994) Identification, cloning, and regulation of a novel endothelial cell protein C/activated protein C receptor. J Biol Chem 269: 26486-26491.

13. Kalafatis M, Rand M, Mann K (1994) The mechanism of inactivation of human factor $V$ and human factor Va by activated protein C. J Biol Chem 269: 31869-31880.

14. Fay P, Smudzin T, Walker F (1991) Activated protein C-catalyzed inactivation of human factor VIII and factor VIIIa. Identification of cleavage sites and correlation of proteolysis with cofactor activity. J Biol Chem 266: 20139-20145.

15. Nicolaes G, Dahlbäck B (2002) Factor V and thrombotic disease: description of a janus-faced protein. Arterioscler Thromb Vasc Biol 22: 530-538.

16. Broekmans AW, Veltkamp JJ, Bertina RM (1983) Congenital protein C deficiency and venous thromboembolism. A study of three Dutch families. N Engl J Med 309: 340-344.

17. Griffin JH, Evatt B, Zimmerman TS, Kleiss AJ, Widerman C (1981) Deficiency of protein C in congenital thrombotic disease. J Clin Invest 68: 1370-1373.

18. Marlar RA, Montgomery RR, Broekmans AW (1989) Diagnosis and treatment of homozygous protein $\mathrm{C}$ deficiency. Report of the Working Party on Homozygous Protein C Deficiency of the Subcommittee on Protein C and Protein S, International Committee on Thrombosis and Haemostasis. J Pediatr 114: 528-534.

19. Seligsohn U, Berger A, Abend M, Rubin L, Attias D, et al. (1984) Homozygous protein C deficiency manifested by massive venous thrombosis in the newborn. N Engl J Med 310: 559-562. 
20. Murakami K, Okajima K, Uchiba M, Johno M, Nakagaki T, et al. (1996) Activated protein C attenuates endotoxin-induced pulmonary vascular injury by inhibiting activated leukocytes in rats. Blood 87: 642647.

21. Zlokovic B, Zhang C, Liu D, Fernandez J, Griffin J, et al. (2005) Functional recovery after embolic stroke in rodents by activated protein C. Ann Neurol 58: 474-477.

22. Loubele ST, Spek CA, Leenders P, van Oerle R, Aberson HL, et al. (2009) Activated protein C protects against myocardial ischemia/reperfusion injury via inhibition of apoptosis and inflammation. Arterioscler Thromb Vasc Biol 29: 1087-1092.

23. Fernandez JA, Xu X, Liu D, Zlokovic BV, Griffin JH (2003) Recombinant murine activated protein C is neuroprotective in a murine ischemic stroke model. Blood Cells Mol Diseases 30: 271-276.

24. Taylor Fj, Chang A, Esmon C, D’Angelo A, Vigano-D’Angelo S, et al. (1987) Protein C prevents the coagulopathic and lethal effects of Escherichia coli infusion in the baboon. J Clin Invest 79: 918-925.

25. Shibata M, Kumar SR, Amar A, Fernandez JA, Hofman F, et al. (2001) Anti-inflammatory, antithrombotic and neuroprotective effects of activated protein $\mathrm{C}$ in a murine model of focal ischemic stroke. Circulation 103: 1799-1805.

26. Bae J, Yang L, Rezaie A (2007) Receptors of the protein C activation and activated protein C signaling pathways are colocalized in lipid rafts of endothelial cells. Proc Natl Acad Sci USA 104: 2867-2872.

27. Mosnier LO, Zlokovic BV, Griffin JH (2007) The cytoprotective protein C pathway. Blood 109: 3161 3172.

28. Casu B (2005) Structure and Active Domains of Heparin. In: Garg H, Linhardt R, Hales C, editors. Chemistry and Biology of Heparin and Heparan Sulfate. 1 ed. Kidlington: Elsevier Ltd. pp. 1-28.

29. Cox M, Nelson D (2008) Lehninger, Principles of Biochemistry. W.H. Freeman \& Company. pp. 1158.

30. Gray E, Mulloy B, Barrowcliffe T (2008) Heparin and low-molecular-weight heparin. Thromb Haemost 99: 807-818.

31. Rosenberg R (1975) Actions and interactions of antithrombin and heparin. N Engl J Med 292: 146151.

32. Mannucci P, Tuddenham E (2001) The hemophilias--from royal genes to gene therapy. N Engl J Med 344: 1773-1779.

33. Srivastava A, Brewer A, Mauser-Bunschoten E, Key N, Kitchen S, et al. (2013) Guidelines for the management of hemophilia. Haemophilia 19: e1-e47.

34. Libby P (2002) Inflammation in atherosclerosis. Nature 420: 868-874.

35. Schram M, Stehouwer C (2005) Endothelial dysfunction, cellular adhesion molecules and the metabolic syndrome. Horm Metab Res 37 Suppl 1: 49-55.

36. Ross R (1999) Atherosclerosis--an inflammatory disease. N Engl J Med 340: 115-126.

37. Borissoff J, Spronk H, Ten Cate H (2011) The hemostatic system as a modulator of atherosclerosis. N Engl J Med 364: 1746-1760.

38. Hausenloy D, Yellon D (2013) Myocardial ischemia-reperfusion injury: a neglected therapeutic target. J Clin Invest 123: 92-100.

39. Turer A, Hill J (2010) Pathogenesis of myocardial ischemia-reperfusion injury and rationale for therapy. Am J Cardiol 106: 360-368.

40. Angus D, van der Poll T (2013) Severe sepsis and septic shockvere sepsis and septic shock. N Engl J Med 369: 840-851.

41. Adhikari N, Fowler R, Bhagwanjee S, Rubenfeld G (2010) Critical care and the global burden of critical illness in adults. Lancet 376: 1339-1346.

42. Bone R, Balk R, Cerra F, Dellinger R, Fein A, et al. (1992) Definitions for sepsis and organ failure and guidelines for the use of innovative therapies in sepsis. The ACCP/SCCM Consensus Conference 
Committee. American College of Chest Physicians/Society of Critical Care Medicine. Chest 101: 1644-1655.

43. Chen Q, Ye L, Jin Y, Zhang N, Lou T, et al. (2012) Circulating nucleosomes as a predictor of sepsis and organ dysfunction in critically ill patients. Int J Infect Dis 16: e558-564.

44. Zeerleder S, Stephan F, Emonts M, de Kleijn E, Esmon C, et al. (2012) Circulating nucleosomes and severity of illness in children suffering from meningococcal sepsis treated with protein C. Crit Care Med 40: 3224-3229.

45. Kutcher M, Xu J, Vilardi R, Ho C, Esmon C, et al. (2012) Extracellular histone release in response to traumatic injury: implications for a compensatory role of activated protein C. J Trauma Acute Care Surg 73: 1389-1394.

46. Luger K, Mäder A, Richmond R, Sargent D, Richmond T (1997) Crystal structure of the nucleosome core particle at 2.8 A resolution. Nature 389: 251-260.

47. Fuchs T, Brill A, Duerschmied D, Schatzberg D, Monestier M, et al. (2010) Extracellular DNA traps promote thrombosis. PNAS 107: 15880-15885.

48. Brinkmann V, Reichard U, Goosmann C, Fauler B, Uhlemann Y, et al. (2004) Neutrophil extracellular traps kill bacteria. Science 303: 1532-1535.

49. Hirsch J (1958) Bactericidal action of histone. J Exp Med 108: 925-944.

50. Xu J, Zhang X, Pelayo R, Monestier M, Ammollo C, et al. (2009) Extracellular histones are major mediators of death in sepsis. Nat Med 15: 1318-1321.

51. Saffarzadeh M, Juenemann C, Queisser M, Lochnit G, Barreto G, et al. (2012) Neutrophil extracellular traps directly induce epithelial and endothelial cell death: a predominant role of histones. PLoS One 7: e32366.

52. Chaput C, Zychlinsky A (2009) Sepsis: the dark side of histones. Nat Med 15: 1245-1246.

53. Kleine T, Lewis P, Lewis S (1997) Histone-induced damage of a mammalian epithelium: the role of protein and membrane structure. Am J Physiol 273: C1925-1936.

54. Fuchs T, Bhandari A, Wagner D (2011) Histones induce rapid and profound thrombocytopenia in mice. Blood 118: 3708-3714.

55. Gamberucci A, Fulceri R, Marcolongo P, Pralong W, Benedetti A (1998) Histones and basic polypeptides activate $\mathrm{Ca}^{2+}$ /cation influx in various cell types. Biochem J 331: 623-630.

56. Xu J, Zhang X, Monestier M, Esmon N, Esmon C (2011) Extracellular histones are mediators of death through TLR2 and TLR4 in mouse fatal liver injury. J Immunol 187: 2626-2631.

57. Gale AJ, Sun X, Heeb MJ, Griffin JH (1997) Nonenzymatic anticoagulant activity of the mutant serine protease Ser360Ala-activated protein C mediated by factor Va. Protein science 6: 132-140.

58. Nicolaes GAF, Bock PE, Segers K, Wildhagen KCAA, Dahlbäck B, et al. (2010) Inhibition of thrombin formation by active site mutated (S360A) activated protein C. J Biol Chem 285: 22890-22900.

59. Mosnier LO, Gale AJ, Yegneswaran S, Griffin JH (2004) Activated protein C variants with normal cytoprotective but reduced anticoagulant activity. Blood 104: 1740-1744.

60. Bae J, Yang L, Rezaie A (2008) Lipid raft localization regulates the cleavage specificity of protease activated receptor 1 in endothelial cells. J Thromb Haemost 6: 954-961.

61. Gale A, Tsavaler A, Griffin J (2002) Molecular characterization of an extended binding site for coagulation factor Va in the positive exosite of activated protein C. J Biol Chem 277: 28836-28840.

62. Manithody C, Fay P, Rezaie A (2003) Exosite-dependent regulation of factor VIIIa by activated protein C. Blood 101: 4802-4807.

63. Mosnier LO, Yang XV, Griffin JH (2007) Activated protein C mutant with minimal anticoagulant activity, normal cytoprotective activity, and preservation of thrombin activable fibrinolysis inhibitordependent cytoprotective functions. J Biol Chem 282: 33022-33033. 
64. Wildhagen K, García de Frutos P, Reutelingsperger C, Schrijver R, Aresté C, et al. (2014) Nonanticoagulant heparin prevents histone-mediated cytotoxicity in vitro and improves survival in sepsis. 2014 123: 1098-1101. 


\section{Chapter 2}

\section{The structure-function relationship of activated protein C: lessons from natural and engineered mutations}

Karin C.A.A. Wildhagen ${ }^{1}$, Esther Lutgens ${ }^{2,3}$, Sarah T.G.B. Loubele ${ }^{1}$, Hugo ten Cate $^{1}$ and Gerry A.F. Nicolaes ${ }^{1}$

1. Department of Biochemistry, Cardiovascular Research Institute Maastricht, Maastricht University, Maastricht, The Netherlands.

2. Department of Pathology, Cardiovascular Research Institute Maastricht, Maastricht University, Maastricht, The Netherlands.

3. Institute for Cardiovascular Prevention (IPEK), Ludwig-Maximilians University Munich, Munich, Germany. 


\section{Abstract}

Protein $\mathrm{C}$ is the central enzyme of the natural anticoagulant pathway and its activated form APC (activated protein C) is able to proteolyse non-active as well as active coagulation factors V and VIII. Proteolysis renders these cofactors inactive, resulting in an attenuation of thrombin formation and overall down-regulation of coagulation. Presences of the APC cofactor, protein S, thrombomodulin, endothelial protein C receptor and a phospholipid surface are important for the expression of anticoagulant APC activity. Notably, APC also has direct cytoprotective effects on cells: APC is able to protect the endothelial barrier function and expresses anti-inflammatory and antiapoptotic activities. Exact molecular mechanisms have thus far not been completely described but it has been shown that both the protease activated receptor 1 and EPCR are essential for the cytoprotective activity of APC. Recently it was shown that also other receptors like sphingosine 1 phosphate receptor 1, Cd11b/CD18 and tyrosine kinase with immunoglobulin-like and EGF-like domains 2 are likewise important for APC signalling. Mutagenesis studies are being performed to map the various APC functions and interactions onto its 3D structure and to dissect anticoagulant and cytoprotective properties. The results of these studies have provided a wealth of structure-function information. With this review we describe the state-of-the-art of the intricate structurefunction relationships of APC, a protein that harbours several important functions for the maintenance of both humoral and tissue homeostasis. 


\section{Introduction}

Protein C (PC) is a vitamin $\mathrm{K}$-dependent serine protease of $62 \mathrm{kDa}$, which is present in human plasma at an average concentration of $70 \mathrm{nM}$ (1). PC circulates in plasma as an inactive zymogen and is activated by thrombin bound to the endothelium associated transmembrane receptor thrombomodulin (TM). TM-bound thrombin removes the 158-169 activation peptide of PC via cleavage at R169, thus generating activated PC (APC). The catalytic activity of the thrombin-TM complex is 20-fold higher when PC is bound to the endothelial protein $\mathrm{C}$ receptor (EPCR) on the surface of vascular endothelial cells (2). Both the interactions of PC with the thrombin-TM complex and with EPCR are $\mathrm{Ca}^{2+}$-dependent. Once PC is activated, APC has anticoagulant as well as cytoprotective functions, which will be described in detail in the next paragraphs.

Two-dimensional (2D) and three-dimensional (3D) structural protein analyses have shown that PC has a mosaic domain structure containing a $\mathrm{N}$-terminal $\gamma$-carboxyglutamic acid (Gla)-domain (residues 1-45), including a helical aromatic segment (residues 3845), two epidermal growth factor (EGF)-like domains (residues 46-92 and 93-136), a linking peptide (residues 137-157), a small activation peptide (residues 158-169) and a trypsin-like serine protease domain (residues 170-419) which harbours the active site triad composed of the residues D257, H211 and S360 (1, 3, 4). Residue numbers for all proteins mentioned refer only to the mature human protein with the exception for the catalytic domain of PC, where chymotrypsin-based residue numbers are given in parentheses. During post-translational processing the single chain PC is cleaved between residues 157 and 158 in the Golgi apparatus, and next the amino acids 157 and 156 are removed by a carboxypeptidase B. The resulting fully processed PC circulates as a heterodimer consisting of a $41 \mathrm{kDa}$ heavy chain and a $21 \mathrm{kDa}$ light chain that are held together via a single disulphide bond (3). Both heavy and light chains carry $N$-linked carbohydrates at residues 97, 248 (93), 313 (150) and 329 (166) (5).

It has been demonstrated that small amounts of APC circulate in human plasma at a concentration of around $40 \mathrm{pM}$ (1). With the half-life of APC being approximately 20 minutes, this suggests that there is a continuous formation of minute quantities of APC. Down regulation of APC activity in plasma occurs via inhibition by $\alpha 2$-macroglobulin and by the serine protease inhibitors (serpins) $\alpha 1$-antitrypsin (AAT), protein $\mathrm{C}$ inhibitor (PCI) and $\alpha 2$-antiplasmin $(6,7)$. Recently it is shown that APC activity can also be inhibited by $\mathrm{Zn}^{2+}$ in purified systems (8).

Although identified and initially characterised as an anticoagulant $(9,10)$, recent interest in PC focuses more on the cellular functions of this protease. In the following sections both functions of PC, i.e. anticoagulant and cytoprotective, will be detailed. 


\section{Anticoagulant function of $A P C$}

PC is the central enzyme of the natural anticoagulant pathway that helps to control thrombin formation. APC generated by the thrombin-TM complex is able to proteolyse the active coagulation factors $\mathrm{V}(\mathrm{FVa}$ ) and VIII (FVIIIa) (Figure 1A). Important cofactors in the inactivation of FVa and FVIIIa are respectively protein $\mathrm{S}$ and factor $\mathrm{V}(\mathrm{FV})$. APC can inactivate FVa by proteolysis of three different peptide bonds at positions R306, R506 and R679 (11-13). The cleavage at R506 is kinetically favoured, protein S-independent and yields a FVa intermediate with decreased factor X (FX) a-cofactor activity (12). The slower cleavage at R306 is stimulated by protein $\mathrm{S}$ and completely inactivates FVa. The exact mechanism through which protein $\mathrm{S}$ appears to stimulate cleavage at R306 in FVa remains unclear. It has been proposed that upon binding of protein S to APC the protease domain of APC undergoes a rotational and/ or translational movement, thereby decreasing the distance of APC's active site to the membrane $(14,15)$. This hypothesis has been challenged, however, by the observation that both R306 and R506 appear to be present at $\sim 80 \AA$ from the membrane, whereas only R306 cleavage appears to be dependent upon protein S. Also, mutagenesis studies of FVa in a proposed APC binding site, for cleavage at R306, showed increased rates of APC catalysed FVa inactivation, that are apparently independent of the cleavage site:membrane distance (16), nor does protein S stimulate the binding of APC to FVa (17). The cleavage at R679 plays a minor role in the inactivation of FVa (12). APC targets both free FVa and the prothrombinase complex, with the latter inactivation being two orders of magnitude slower $(12,18)$. APC-catalysed inactivation of FVIIIa, at homologous positions in the FVIII A-domains at R336 and R562 and at a third cleavage site at R740, which can also be cleaved by thrombin $(19,20)$, is stimulated not only by protein $\mathrm{S}$ but also by FV, through an hitherto unknown mechanism (21). It was shown, however, that the presence of the FV B-domain and an R506 cleavage site are required for the cofactor activity of FV in the APC-catalysed inactivation of FVIIIa $(22,23)$. It should be noted that in contrast to FVa, FVIIIa in solution is subject to rapid decay caused by dissociation of the A2 domain from the rest of the molecule. As a result, the majority of FVIIIa activity $(70-80 \%)$ is lost spontaneously (24). Remaining FVIIIa activity is then lost by APC-catalysed cleavage at R336. Thus, the greater part of FVIIIa activity is not lost by proteolytic action of APC but rather by spontaneous FVIIIa subunit dissociation. This observation is likewise illustrated by the apparently several fold lower affinity of APC for FVIIIa, as compared to that of FVa (17).

Resistance to APC, as observed in $\mathrm{FV}_{\text {Leiden }}$, a FV gene with a single missense mutation of $\mathrm{R} 506$ to $\mathrm{Q}$, is the most common genetically determined hypercoagulability disorder in Caucasians $(25,26) . \mathrm{FV}_{\text {Leiden }}$ causes APC resistance through several mechanisms: (i) the absence of the cleavage site at position R506 results in slow inactivation of $\mathrm{FVa}_{\text {Leiden }}$ by cleavage at R306 only; (ii) the absence of a proper binding site for APC, which includes residue R506, blocks the ability of APC to directly inhibit thrombin 
formation by dissociating the $\mathrm{FXaFVa}_{\text {Leiden }}$ complex; and (iii) $\mathrm{FV}_{\text {Leiden }}$ does not enhance APC-catalysed FVIIIa inactivation (27).

The anticoagulant PC pathway is vital to normal haemostasis. Reduced levels of circulating APC are an independent risk factor for both venous and arterial thrombosis. Individuals with heterogeneous PC deficiency have a significantly increased risk to develop thrombosis (28-31). Individuals with homozygous protein $\mathrm{C}$ deficiency may suffer from catastrophical and fatal complications like neonatal purpura fulminans and venous thrombosis already as neonates (32). Even in the presence of aggressive anticoagulant therapy, homozygous PC deficiency can be life threatening (33-35).

\section{Cytoprotective functions of $A P C$}

The widely observed beneficial effects of APC in animal models of sepsis, pulmonary injury, stroke and wound healing (36-44) cannot be explained solely by APC's anticoagulant effects. APC administration, and not anticoagulants like antithrombin III and TFPI $(45,46)$, has been successfully used to reduce mortality in severe sepsis patients (Prowess trial [47]). There is, however, controversy as to the general use of APC in the treatment of severe sepsis and septic shock patients since a recent metaanalysis did not confirm the overall beneficial effects of APC on 28-day survival (48). Collectively these and other data have manifested the dual functions of APC in both anticoagulation and cytoprotection. Protease activated receptor 1 (PAR-1) and EPCR were shown to be required for APC-dependent cytoprotective effects. PAR-1, a transmembrane protein receptor, which is activated upon limited proteolysis, is present on many cell types, including endothelial cells, epithelial cells, myocytes, fibroblasts, astrocytes and neurons (49, 50). Most of these cell types also express EPCR (51-54). APC has cytoprotective effects in different cell types by anti-inflammatory, anti-apoptotic and endothelial barrier stabilising effects. These effects, rather than the anticoagulant activity of APC, likely explain the protective effect of APC administration in animal studies of sepsis, lung injury, myocardial infarction and ischaemic stroke (37-39, 41-44, 55-57). There are still uncertainties with regard to the exact roles that EPCR and PAR1 play in APC-induced cell signalling. Recently, the involvement of other cell-receptors such as Tie2, S1P1, CD11b/CD18, ApoER2 (LRP8) and epidermal growth factor receptor in APC signalling was shown, thereby providing yet additional insights into the molecular mechanism that underlies the cell-protective properties of APC (58-60). APC-induced cytoprotection can be attributed to any of or a combination of a number of cellular responses, including an altered inflammatory response, a modified anti-apoptotic response and/or an improved barrier function of the endothelium. The different cytoprotective effects and molecular mechanisms identified so far are described in detail below and are illustrated in Figure 1B-D. 


\section{Anti-inflammatory activities}

The effects of APC on endothelial cells and leukocytes are summarized in Figure 1B. APC modulates transcription of genes involved in the major inflammatory pathways. In both endothelial cells and leukocytes, APC reduces via PAR-1 and EPCR gene expression levels and functional activity of nuclear transcription factor $\mathrm{kB}(\mathrm{NF \kappa B})$ (61). In leukocytes, APC leads via PAR-1, sEPCR, proteinase-3 (PR3) and CD11b/ CD18 to increased concentrations of sphingosine kinase-1 (Sphk-1) and sphingosine 1 phosphate (S1P), resulting in suppression of pro-inflammatory activity $(55,59)$. Both inhibition of NFKB and upregulation of S1P result in inhibition of cytokine signalling, down-regulation of cell surface adhesion molecules and increased expression of antiinflammatory genes like interleukin 10 (IL-10) (27, 55, 61).

Ultimately this results in reduced leukocyte transmigration through the endothelium and less tissue damage in the underlying tissue (55). Recent results from Kerschen et al. have shown that APC targets EPCR-positive CD8+ dendritic cells in mice, which results in a reduction of mortality after induction of endotoxaemia (62).

\section{Anti-apoptotic activity}

Apoptosis is an intrinsic program of cell death that occurs in various physiological and pathological situations. It can be induced by both the death receptor (extrinsic) pathway and mitochondrial (intrinsic) pathway. Caspase-8 activation in the extrinsic pathway and release of cytochrome $\mathrm{c}$, apoptosis inducing factor (AIF) and pro-caspases in the intrinsic pathway finally lead to caspase-3 activation and apoptosis (55).

Figure 1C shows a summary of the different anti-apoptotic effects of APC on endothelial cells. APC can reduce apoptosis induced by hypoxia, t-PA and staurosporine in different types of endothelial cells $(61,63,64)$. In these experimental conditions, the presence of APC resulted in decreased caspase- 3 and -8 activation, reduced DNA degradation and p53 expression, as well as decreased nuclear translocation of AIF, decreased translocation of phosphatidyl serine to the outer cell membrane and normalisation of the Bax/Bcl-2 ratio $(27,55,65)$. Via PAR-1 cleavage, APC induces phosphorylation of extracellular signal regulated protein kinases $1 / 2$ and members of the mitogen-activated protein kinase family (66), resulting in restraint of the mitochondrial permeability transition pore, inhibition of cytochrome $\mathrm{C}$ release into the cytoplasm and prevention of apoptosis signalling (67).

\section{Protection of the endothelial barrier function}

In healthy individuals, the intact endothelial layer forms the first natural physical barrier that protects underlying tissues from blood-borne pathogens by controlling passage of molecules and migration of leukocytes into and from the tissues. Excessive or 


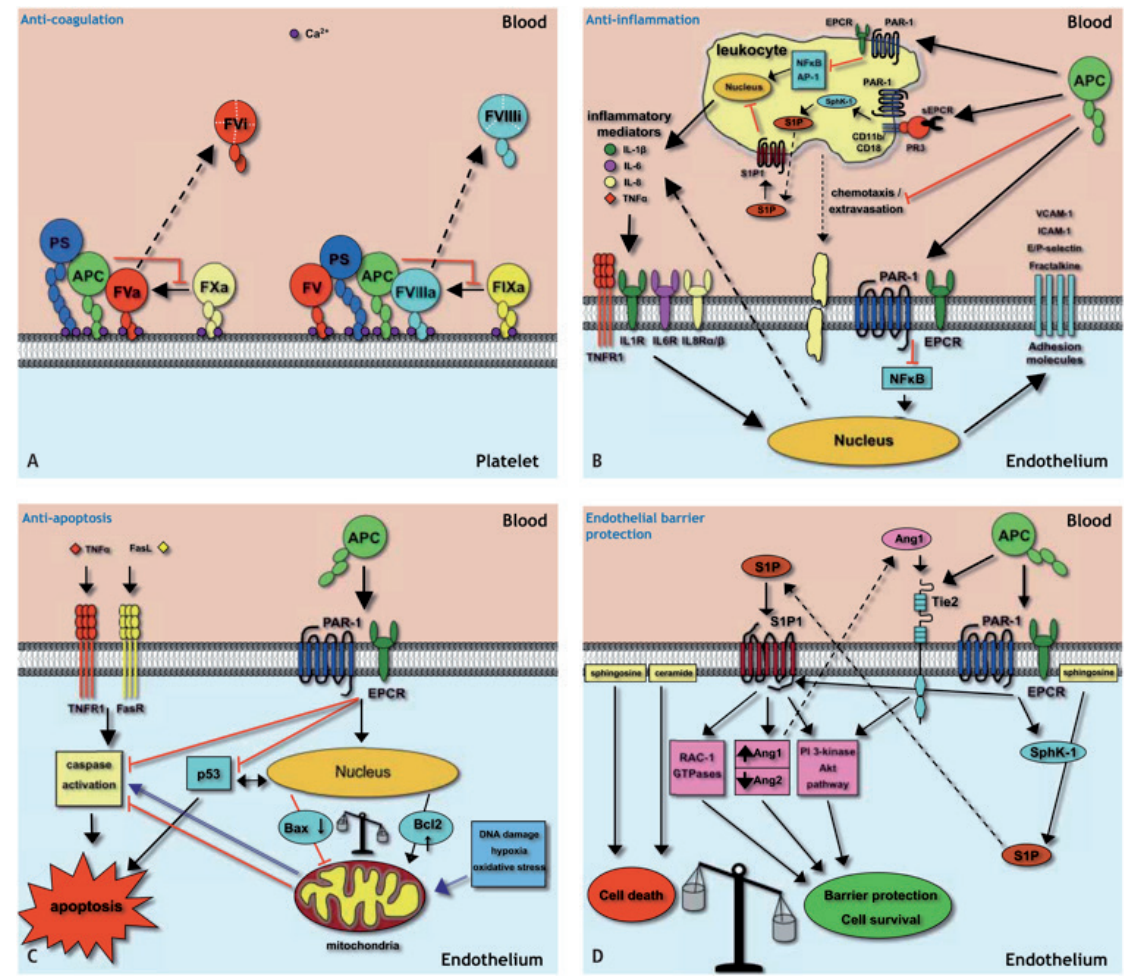

Figure 1: Humoral and cellular effects of APC

A) Anti-coagulation. Upon binding of APC to either FVa or FVIIIa, APC ablates cofactor activity through competition with the respective serine proteases (FXa or FIXa, respectively). Subsequent proteolysis of FVa and FVIIIa renders these cofactors inactive, resulting in irreversible attenuation of thrombin formation and overall downregulation of coagulation. Protein S and FV are important cofactors of APC in this process.

B) Anti-inflammation. PAR-1 and EPCR dependent signalling of APC in endothelial cells and leukocytes inhibits NFKB / AP-1 signalling, resulting in an inhibition of inflammatory mediator release and downregulated expression of adhesion molecules. Binding of APC to sEPCR, PR3 and CD11b/CD18 on leukocytes activates PAR-1, resulting in upregulation of Sphk-1 and S1P. Subsequently binding of S1P to its receptor S1P1 suppresses the pro-inflammatory activity of leukocytes. These effects of APC result in decreased leukocyte chemotaxis and lower infiltration of leukocytes in the endothelium.

C) Anti-apoptosis. Apoptosis can be initiated by binding of TNFa or FasL to death receptors (TNFR1/ FasR) on the cell membrane of endothelial cells (extrinsic pathway) or by DNA damage, hypoxia and oxidative stress that increase the permeability of the mitochondrial outer membrane (intrinsic pathway). Activation of both pathways results in activation of caspases APC inhibits caspase activation, suppresses the expression of pro-apoptotic genes ( $553+\mathrm{Bax}$ ) and upregulates the transcription of anti-apoptotic genes (Bcl2). The suppression of Bax and up-regulation of Bcl2 leads to a normalization of the $\mathrm{Bax} / \mathrm{Bcl}-2$ ratio on the mitochondrial membrane and inhibition of apoptosis.

D) Endothelial barrier protection. APC protects the endothelial barrier function by induction of SphK-1 and up-regulation of the bioactive lipid S1P. Binding of S1P to S1P1, leads to improved cell survival and barrier protection via activation of the PI 3-kinase/Akt pathway, upregulation of both RAC-1 GTPases and Ang1 and downregulation of Ang2. Ang1 leads via binding to Tie2 to further activation of the PI 3-kinase/Akt pathway and enhancement of endothelial barrier integrity. 


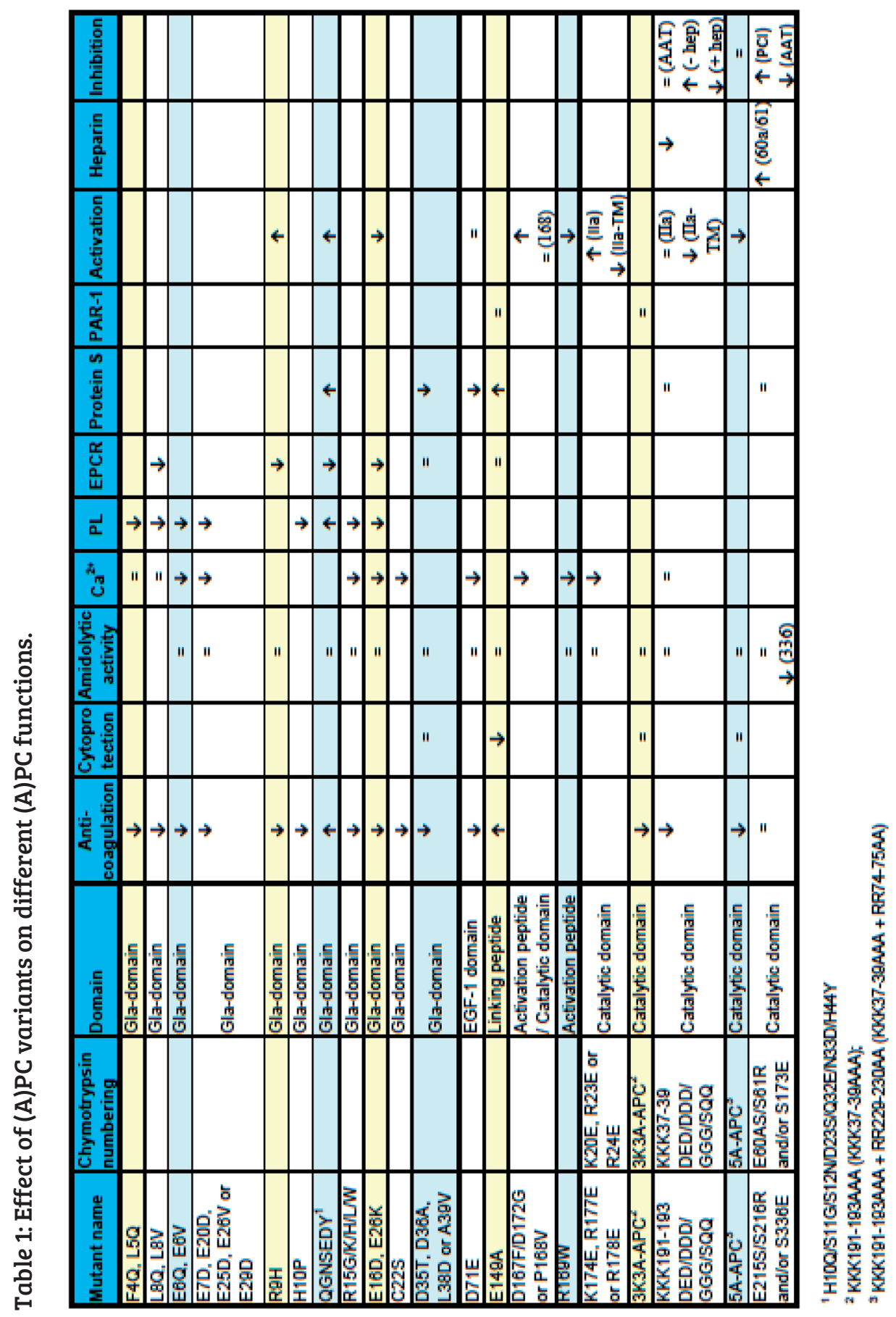



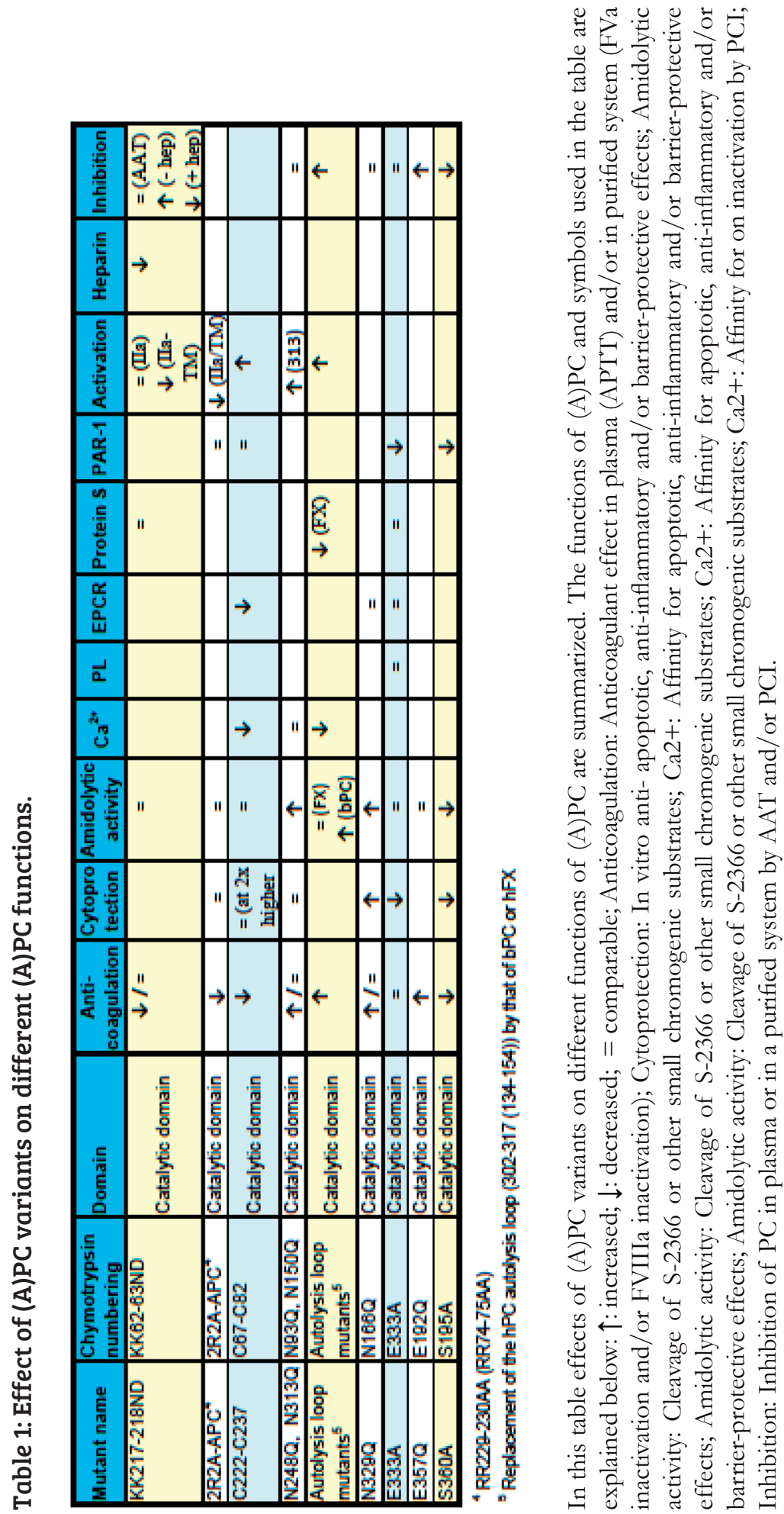
prolonged increases in permeability of the endothelial monolayer barrier, as seen in cases of (chronic) inflammation, may lead to tissue oedema/swelling (68). The effects of APC on endothelial barrier function are summarised in Figure 1D. APC improves the integrity of the endothelial barrier by upregulation of sphingosine kinase-1 (Sphk1), sphinghosine 1 phosphate (S1P), angiopoietin 1 (Ang1), tyrosine kinase with immunoglobulin-like and EGF-like domains 2 (Tie2) and tight junction protein zona occludens (69). APC also stimulates the migration of smooth muscle cells (SMCs) into the endothelium. These SMCs form a sheath around the endothelium that decreases the permeability of the membrane. For APC's barrier stabilising effects, the presence of the receptors EPCR, PAR-1, sphingosine 1 phosphate receptor 1 (S1P1) and Tie2 is required (69). The improved endothelial barrier limits the infiltration of inflammatory cells through the endothelium into the subendothelial space $(55,61,70)$.

\section{Effect of mutations on the function of APC}

The dissection of anticoagulant and cytoprotective properties of APC, as has been demonstrated through mutagenesis studies from several groups $(17,70-77)$, illustrates the different structure-function facets of APC. Several variants have been designed and tested for their functional properties and it appears that specialised surface areas convey different properties to the APC molecule. Meanwhile, numerous natural variants of protein $\mathrm{C}$ have been reported that cause functional defects of the protein. In an attempt to provide a 3D properties map of APC, we have collected available structure-function data of APC and have placed these in the perspective of the 3D structure of the protease, in order to better understand the interactions of APC with its many physiological binding partners. Since the naturally occurring type I deficiencies (decreased PC expression levels with normal specific activity) are of less interest from a structure-function point of view, we have restricted ourselves to type II deficiencies (normal PC expression levels with decreased specific activity). It is important to mention that many PC variants are tested for their respective expression levels, as well as for their amidolytic activities on chromogenic substrates and their activities in plasma or on macromolecular substrates. Measurement of expression levels allows detection of type I deficiencies and measurement of amidolytic activities probes only the consistency of the active site. Thus, even though the enzymatic capacity of APC as such is unaltered, changes in substrate recognition sites (exosites) or in cofactor or membrane binding sites may still result in an overall reduced biological activity of an APC variant. Effects of mutations in different regions of protein $\mathrm{C}$ are described below and summarised in Table 1). In Figure 2, the human PC sequence is shown with important regions and residues indicated to help the reader follow the discussion of different APC variants. Likewise, Figure 3 aids the reader in mapping each of the mentioned variations/ mutations onto a 3D representation of human APC. 


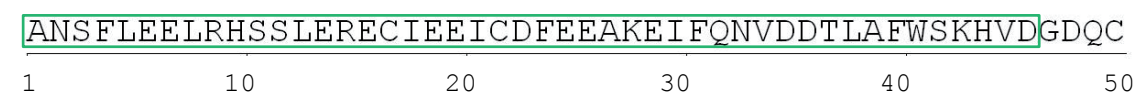

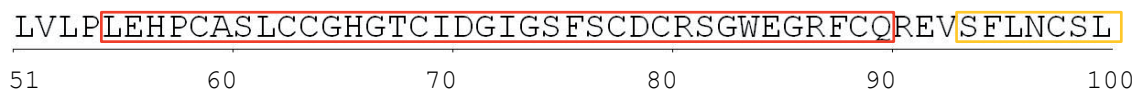

DNGGCTHYCLEEVGWRRCSCAPGYKLGDDLLOCHPAVKFPCGRPWKRMEK

$\begin{array}{lllll}101 & 110 & 120 & 130 & 140\end{array}$

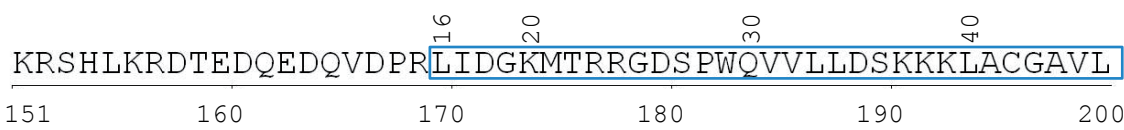

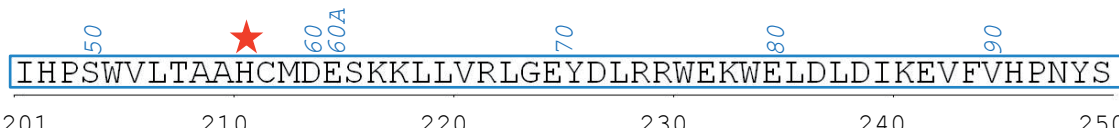

$201210 \quad 220 \quad 230 \quad 240 \quad 250$

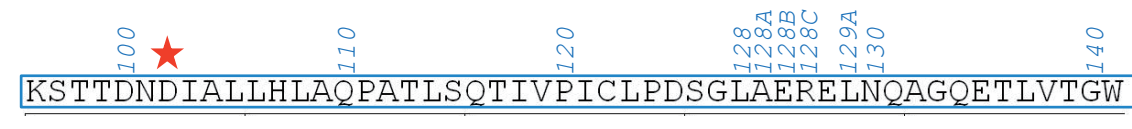

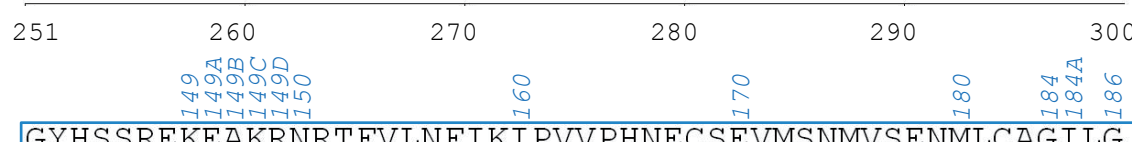

GYHSSREKEAKRNRTEVLNFIKIPVVPHNECSEVMSNMVSENMLCAGILG
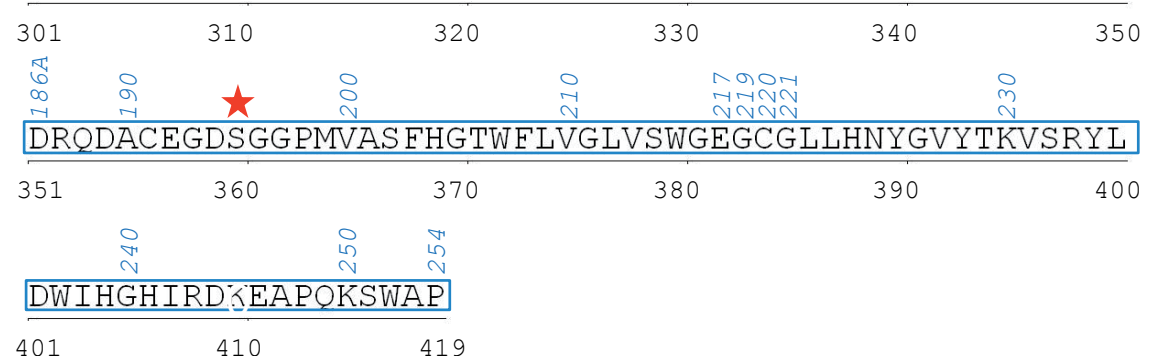

Figure 2: Human protein $\mathrm{C}$ amino acid sequence

Indicated is the amino acid sequence for hPC from the UniProt database (identifier ID: P04070,PROC_HUMAN, ref.4). Domain boundaries are as defined in the UniProt database, Numbering below the sequence represent hat for the mature protein, numbering above the sequence indicates numbering according to the alternative chymotrypsin nomenclature equivalents. Active site residues are indicated by red stars. Boxes indicate the domain boundaries, with color coding as used in Figure 6: green for the Gla-domain, red for EGF-1 domain, orange for EGF-2 domain and blue for the catalytic, serine protease, domain. 


\section{Light chain: Gla-domain mutations}

The N-terminal Gla-domain (residues 1-45) confers PC its membrane and EPCR binding capacity and contributes to the binding between APC and protein S. In general, Gla-domain containing proteins bind to phospholipid (PL) vesicles in at least two stages. First, two or three cation binding-sites in the Gla-domain are occupied, resulting in a conformational change of the Gla-domain. Second, a group of four or five divalent cation binding sites with a high specificity for $\mathrm{Ca}^{2+}$ become occupied, enabling binding of the protein to acidic PL vesicles. A cluster of hydrophobic side chains that extend away from the core of the protein (F4, L5, and L8, see also Figure 4) was shown to be part of a PL binding site (78-80). These side chains are flanked by H10, which was suggested to participate in the formation of a pore in the protein that may be important for the formation of a protein-PL ion pair in aqueous medium $(81,82)$.

In the presence of calcium, seven $\mathrm{Ca}^{2+}$-ions occupy a channel through the Gla-domain of APC. These $\mathrm{Ca}^{2+}$-ions are important for maintenance of the overall structure of the Gla-domain, for the anticoagulant function of APC and the interaction with EPCR (83) (Figure 4). A crystal structure of a complex between the Gla-domain of PC and sEPCR has been determined (84). In this structure, PC binds to sEPCR via hydrogen bonds at Gla-residues 7, 25, 29 and via hydrophobic interactions at residues F4, E7 and L8 to EPCR (84). Notheworthy, these interactions are highly specific for the interaction of the hAPC Gla domain and EPCR, as it was shown that hAPC FX- and prothrombin (PT) Gla-domain chimeras do not exhibit any cytoprotection (85).

Both natural (R9H) and engineered (F4Q, L5Q, L8Q, H10P) APC variants show reduced anticoagulant activities, although they have a normal $\mathrm{Ca}^{2+}$-dependent conformation of the Gla-domain $(78-82,86)$. It is likely that these mutants have either suboptimal PL binding or disorientated binding to the PL membrane which results in poor alignment of the active sites towards the complementary cleavage sites of FV/FVa and FVIII/ FVIIIa $(78,80)$. For variants L8Q and R9H, the reduced affinity for EPCR can contribute to the reduced anticoagulant activity $(78,80,86)$.

To investigate which Gla-residues are essential for the anticoagulant activity of APC, different mutants were expressed wherein the nine E residues of the Gla-domain of APC were substituted (83). Kinetic parameters for chromogenic substrate (S-2366) conversion were normal for all variants. Single mutations of Gla-residues at positions $6,7,16,20,26$ or 29 resulted in APC variants with less than $10 \%$ of the anticoagulant activity of wt-APC in an APTT based assay. E25D-APC expresses $\sim 20 \%$ of wt-APC anticoagulant activity and Gla-residues 14 and 19 do not contribute to anticoagulant function. Furthermore, E16D-PC and E26K-PC were not activated by the thrombinTM complex on endothelial cells and showed no binding to sEPCR (86). Collectively, $\mathrm{Ca}^{2+}$ interactions are essential for the anticoagulant functions of APC and are influenced by mutations in different regions of the Gla-domain $(83,87,88)$. 


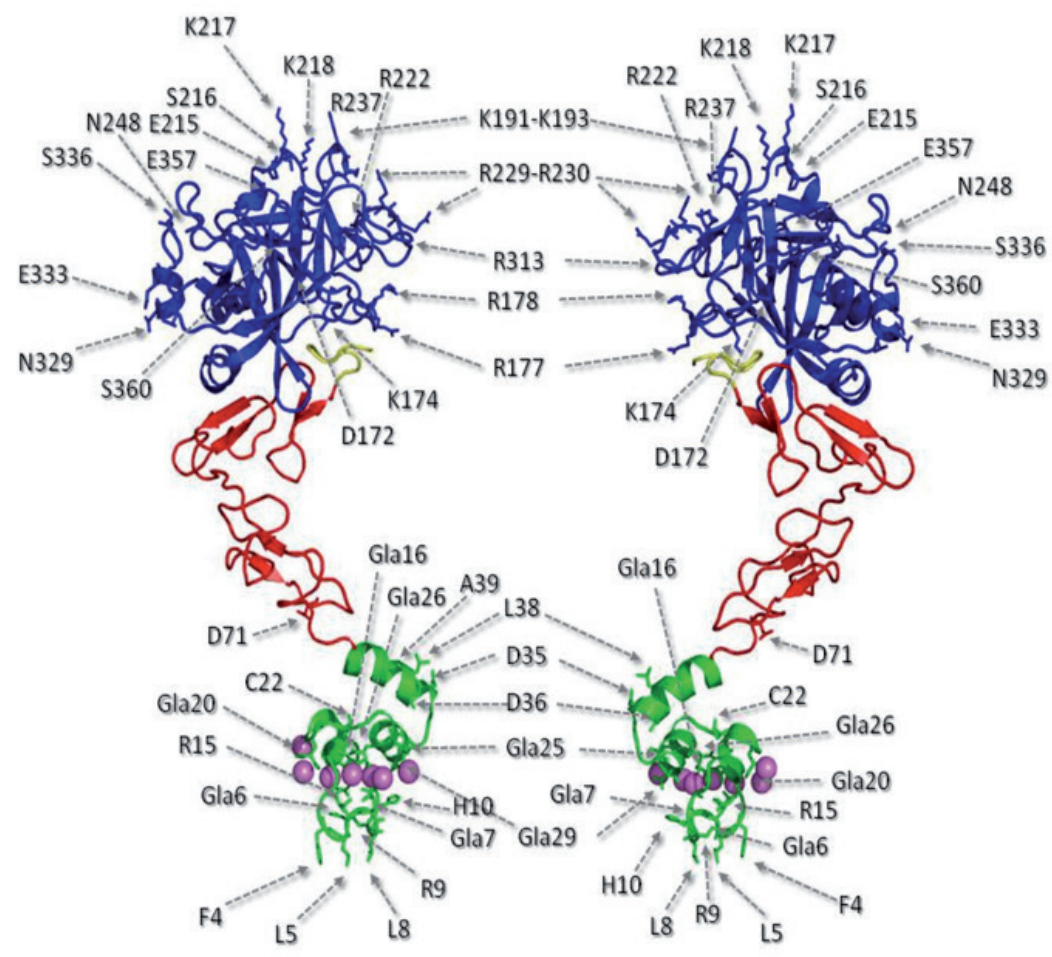

Figure 3: Mapping of APC mutations on its overall 3D structure

The mosaic protein architecture of APC is shown, with the left representation being 180 degrees rotated along a vertical Y-axis. The different domains are shown in their ribbon structure. From $\mathrm{N}$ terminus to C-terminus are shown: green, the Gladomain, residues 1-37, residue numbers refer to the mature protein) including a helical aromatic segment, residues 38-45; red, EGF-1 and EGF-2 domains, residues 46-92 and 93-136 respectively; yellow, a linking peptide (residues 137-157); blue, the trypsin-like serine protease domain, residues 170-419 (16-254). Side chains are shown for those residues that are mentioned in the text to indicate their approximate position in the protein.

These aforementioned results are in agreement with the analysis of PC that was isolated from type II PC deficient patients. Both the E7D $(89,90)$ and E20A/V34M (Protein C Vermont, [91]) are associated with thrombotic complications, despite the fact that carriers of these mutations have normal PC antigen levels and APC amidolytic activities. Site directed mutagenesis of R15 shows that this residue is critical for the $\mathrm{Ca}^{2+}$ dependent conformation of the Gla-domain required for PL binding and hence for APC's anticoagulant activity (92). In agreement with this is the fact that individuals who carry a mutation at R15 (R15W or R15G, with the latter also known as PC Yonago) have an increased thrombotic tendency $(93,94)$. Mutation of hexapeptide disulfide loop residue $\mathrm{C} 22$ to serine, results in a complete loss of anticoagulant activity in an APTT based clotting assay and less than $5 \%$ of the activity of wt-APC toward inactivation of 
FVIIIa (87, 88). Residues R15, C22 and Gla-residues 6, 7, 16, 20, 25, 26 and 29 are thus absolutely required for APC's anticoagulant function.

The C-terminus of the Gla-domain (residues 25-45) and particularly D35, D36, L38 and A39 were shown to interact with protein S (79). Each of these four residues was individually replaced by the corresponding PT amino acid residue to investigate their functional importance (95). The amidolytic activities and anticoagulant properties of these mutants were not severely affected in the presence of protein S, except for the L38D mutant which exhibited 20-fold impaired anticoagulant activity as compared to wt-APC in plasma or in protein S dependent assays using purified proteins. Notably, in the absence of protein S, L38D-APC was indistinguishable from wt-APC. L38D-APC binds EPCR with equal binding affinity as wt-APC. All mutants tested in this study (95) inhibited apoptosis to a similar degree and had similar barrier-protective capacities as wt-APC in the absence of protein $\mathrm{S}$.

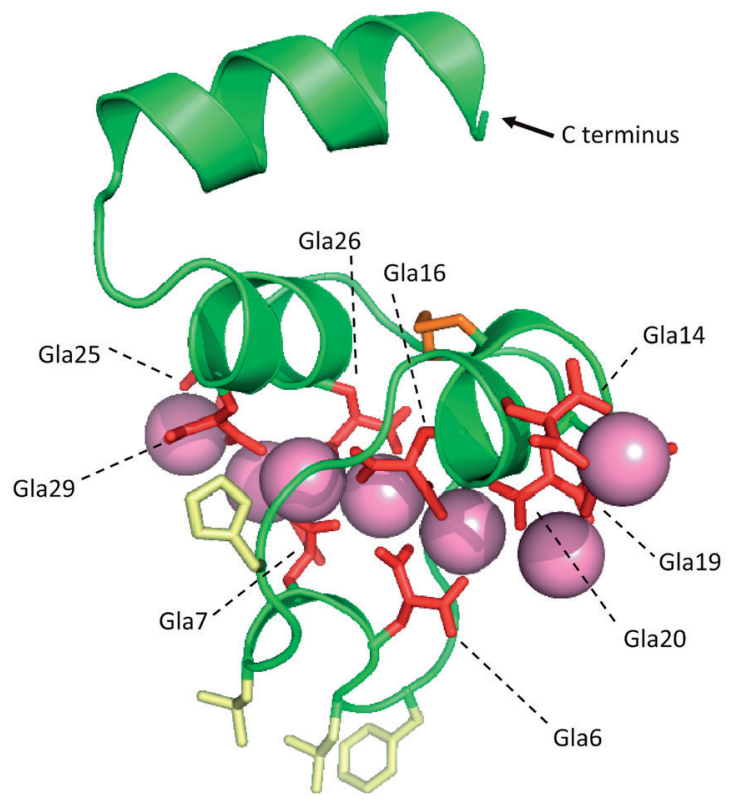

Figure 4: 3D structure of the APC-Gla-domain

3D structural model of the human APC-Gla-domain (residues 1-45), represented as a ribbon diagram with helix and loop regions visible of the published crystal structure for the APC Gla-domain (residues 1-33) (84) onto which the helical aromatic segment at the $C$ terminus of the domain is manually grafted (coordinates for residues 34-45 were kindly provided by Dr. Villoutreix, University of Paris 7, France) according to the overall orientation from the crystal structure for the bovine prothrombin Gla-domain (123). The side chains of Gla-residues are shown in red with calcium ions represented as pink spheres. The amino acid side chains of residues F4, L5, L8 and H10, involved in formation of a PL binding site are shown in yellow. In orange the hexapeptide disulphide loop is shown which connects residues C17 and C22. The C-terminus of this fragment, which connects to the helical segment and EGF-1 domain, is indicated. For orientation, Gla residue numbers are given. 


\section{Light chain: Mutations in EGF domains and linking peptide}

The EGF domains of APC have been described to be involved in the interaction of APC with $\mathrm{Ca}^{2+}$ and protein S (96-98). In D71E-APC, in which the $\beta$-hydroxyaspartic acid was replaced by glutamate, a $\mathrm{Ca}^{2+}$-dependent epitope for the interaction with protein $\mathrm{S}$ in the EGF-1 domain was lost, resulting in only $10 \%$ of the anticoagulant activity of wt-APC (97).

The so-called "linking peptide" (residues 137-157) precedes the activation peptide, which is excised upon PC activation. A special position here is taken by E149. Mutagenesis of this residue into alanine resulted in a three-fold increased anticoagulant activity in an APTT-based clotting assay (75). In an amidolytic assay, probing the conversion of a small chromogenic substrate, and in the absence of protein $\mathrm{S}$, no difference between E149A-APC and wt-APC was observed. In thrombin generation and FVa inactivation studies, E149A-APC required lower concentrations of protein $\mathrm{S}$ than wt-APC to achieve half-maximal effects. Taken together, E149A-APC expresses increased anticoagulant activity in the presence of protein S, likely through enhanced affinity for protein S. The improved anticoagulant effect was verified in an in vivo murine thrombosis model (75). Remarkably, the cytoprotective effects of E149A-APC were severely diminished, despite a normal cleavage of PAR-1 and normal binding to EPCR (75). E149A-APC expressed only $6 \%$ of the anti-apoptotic activity of wt-APC in a staurosporine-induced apoptosis model in endothelial cells and was unable to down-regulate IL-6 release in lipopolysaccharide (LPS) treated U937 monocytes (75). The molecular mechanism which reconciles the loss of cytoprotective effects, while amidolytic activities and PAR1 and EPCR-binding are normal, is at present not available.

\section{Heavy chain: Mutations in the activation peptide and $C$ terminus of the catalytic domain}

Since the activation peptide (residues 158-169) is excised during PC activation, mutagenesis in this region is expected to affect $\mathrm{PC}$ activation. Three variants have been studied: D167F/D172G-PC, P168V-PC and R169W-PC (96, 99, 100). At saturating $\mathrm{Ca}^{2+}$ concentrations, the activation rates of D167F/D172G and P168V mutants and wt-PC by the thrombin-TM complex were comparable, but the mutants required fourfold higher $\mathrm{Ca}^{2+}$ concentrations than wt-APC to achieve half-maximal activation rates. When only thrombin was present, $\mathrm{Ca}^{2+}$ was not able to influence the activation of the D167F/D172G and P168V mutants, though $\mathrm{Ca}^{2+}$ effectively inhibits activation of wt-PC by thrombin (96). R169W-PC can not be activated by thrombin, but can be efficiently activated by chymotrypsin (100). Residues in close proximity of R169 appear to influence $\mathrm{Ca}^{2+}$ affinity and/or the $\mathrm{Ca}^{2+}$-dependent conformation of PC through either direct or indirect mechanisms.

Probing this same region, residues K174, R177 and R178 were mutated to glutamic acid. The activation rates of the mutants by thrombin were 12 -fold faster than that 
observed for wt-PC in the presence of $\mathrm{Ca}^{2+}$, and unchanged in the absence of $\mathrm{Ca}^{2+}$. Addition of TM did not stimulate activation of the PC variants, suggesting that residues 174, 177 and 178 are involved in PC activation by the thrombin-TM complex (101).

\section{Heavy chain: catalytic domain mutations}

The well-studied catalytic domain of APC, residues 170-419 (16-254: chymotrypsin numbering is given in parentheses from hereon), contains the catalytic triad, which is responsible for the proteolysis of APC-substrates. Moreover this domain contains substrate binding regions, to which APC substrates must bind prior to their cleavage. Mutations in this domain are therefore likely to directly influence the catalytic efficiency and the binding of substrates to the protease. The 3D structure of the catalytic domain and its various loop regions is shown in Figure 5.

\section{Mutations in 191-loop (37-loop), Cat -binding loop and autolysis loop}

For the anticoagulant activity of APC, especially the cleavage at R506 in FVa is required $(73,102)$. Protein-protein docking- and mutagenesis studies have revealed that multiple electrostatic contacts between APC and the R506 cleavage site in FVa mediate the efficient contact between FVa and APC $(16,102)$. The positively charged area in the catalytic domain of APC includes the 191-loop, alternatively described as 37-loop (residues 190-193 (36-39)), the $\mathrm{Ca}^{2+}$-binding loop (residues 225-235 (70-80)) and the autolysis loop (residues 302-317 (143-154)) (3, 103). The importance of the electrostatic interactions made by these surface loops was shown by several mutagenesis studies.

Three 191-loop (37-loop) and/or $\mathrm{Ca}^{2+}$-binding loop mutants were constructed: 2R2AAPC (RR229/230AA (RR74-75AA)), 3K3A-APC (KKK191-193AAA (KKK3739AAA)) and 5A-APC (combination of both mutants) (73, 74, 102, 104-106). In an APTT-based assay the anticoagulant activities of 2R2A-APC, 3K3A-APC and 5A-APC were $14 \%, 5 \%$ and $<3 \%$, respectively, of that of wt-APC, with amidolytic activities being unaffected. These effects can largely be explained by reduced cleavage at R506 in FVa $(73,74,102)$, while these residues are also important for FVIIIa inactivation at both R336 and R562 in FVIIIa (107). In vivo, 5A-APC lacked antithrombotic activity in an acute arterial thrombosis model (75). Thus these engineered APC variants, and in particular the 5A-mutant, show reduced anticoagulant activity while the in vitro and in vivo cytoprotective effects were undistinguishable from wt-APC, with normal affinity for EPCR and PAR-1 (72-75).

The 191-loop (37-loop) is furthermore established as a heparin-binding loop (104, 105). Mutation of residues KKK191-193 (KKK37-39) results in reduced heparin stimulated inhibition by PCI. Activation and $\mathrm{Ca}^{2+}$-dependent inhibition of 191-loop (37-loop) variants by thrombin was identical to wt-PC. However activation of these PC 
variants by the thrombin-TM-complex was severely impaired (105). This indicates that the positively charged residues KKK191-193 (KKK37-39) contribute to PC activation by the thrombin-TM complex, to the anticoagulant function of APC and are part of a heparin-binding site. In line with this, a K193Q (K39Q) mutation has been described in a type II deficient patient with a history of thrombo-embolic disease (108).

Mutation of R229 to glutamine (R74Q) has been described in a family with type II PC deficiency, associated with thrombosis. The affected individuals have normal PC antigen and amidolytic activity levels, but $\sim 50 \%$ decreased anticoagulant activity. These data, together with those from structure-function studies of recombinant R229Q (R74Q) PC, indicate the importance of R229 (R74) for anticoagulation and activation of PC by the thrombin-TM complex $(89,90,109-111)$.

The PC autolysis loop (residues 302-317 (143-154)) is involved in stabilisation of the activation peptide in its $\mathrm{Ca}^{2+}$-dependent inhibitory conformation, leading to enhanced activation by the thrombin-TM-complex and decreased activation by thrombin (112, 113). The hPC autolysis loop is five residues longer than the autolysis loops of other vitamin $\mathrm{K}$-dependent proteases and four residues longer than the homologous loop in bPC. Replacement of the autolysis loop in hAPC by the shorter bovine form resulted in a four-fold increased catalytic activity. Replacement of the hAPC autolysis loop by that of $\mathrm{hFXa}$ resulted in higher $\mathrm{Ca}^{2+}$-affinity and increased PC activation by thrombin both in the absence (5-fold) and presence (2.5-fold) of TM. Furthermore, from the reduced half-life of these PC variants in plasma it was concluded that the autolysis loop is involved in inhibition by serpins. In both chimeras, substitution of the autolysis loop results in increased inactivation rates of FVa and FVIIIa, while conflicting results were reported for interaction with protein $\mathrm{S}(112,113)$. Studies wherein individual residues of the autolysis loop were mutated $(114,115)$ showed that APC residues R306 (R147), K308 (K149), K311 (K149c), R312 (R149d) and R314 (R151) are important for FVa cleavage at R506 and residues R306 (R147), K311 (K149c) and R314 (R151) are essential for FVIIIa inactivation by APC.

\section{Mutations in 214-loop (60-loop)}

bAPC is, in contrast to its human counterpart, nearly completely resistant to inactivation by AAT. Homology modelling and site- directed mutagenesis studies of the serine protease domains of bAPC and hAPC identified the 214-loop (residues 214-220) (60loop (residues 60-65)) as being probably involved in interaction with AAT, heparin and PCI (116). Three PC mutants, E215S/S216R (E60aS/S61R), S336E (S173E) and K217N/K218D (K62N-K63D) were generated (104, 116, 117). Mutant APC amidolytic activities were unaffected, except for S336E (S173E) which had a reduced Km, which suggests that a glutamate residue at position 336 (173) improves the fit of the substrate in the catalytic cleft $(104,116,117)$. K217N/K218D (K62N-K63D)-APC had slightly lower anticoagulant activity compared to wt-APC and showed undetectable binding 


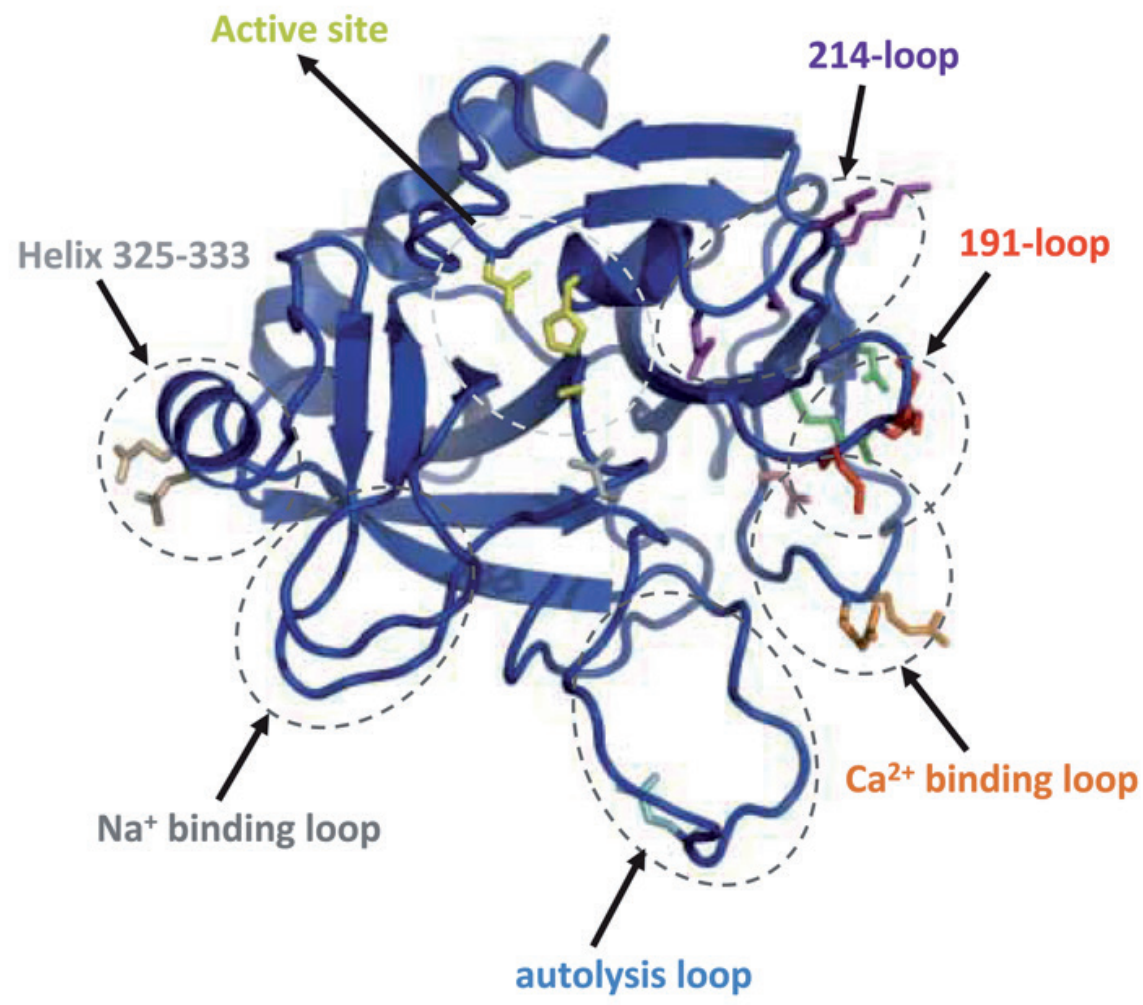

Figure 5: 3D structure of the APC serine protease domain

The serine protease domain is represented as a ribbon diagram with helix and loop regions visible in dark blue. Relevant loops and residue side chains have been indicated. Yellow: H211 (H57), D257 (D102), S360 (S195) active site residues; Red: K191 (K37), K192 (K38), K193 (K39) in the 191-loop (37-loop); Orange: R229 (R74), R230 (R75) in the $\mathrm{Ca}^{2+}$-binding loop; Light blue, E309 (E149A) in the autolysis loop; Wheat: E330 (E167) and E333 (E170) in helix 325-333 (162-170); Purple: E215 (E60a), S216 (S61), K217 (K62), K218 (K63) in the 214-loop (60-loop); Green: R222 (R67) and D237 (D82), both residues after mutation to $\mathrm{C}$ are involved in the formation of an engineered disulphide bridge; Gray: E357 (E192). The position of the $\mathrm{Na}^{+}$-binding loop, at V221-E225 (V66-E70), is indicated.

to heparin $(104,117)$. Both E215S/S216R (E60aS/S61R)-APC and S336E (S173E)APC are resistant to AAT (116). E215S/S216R (E60aS/S61R)-APC was 2-3 times more efficiently inhibited by PCI than wt-APC in the absence as well as the presence of heparin (116). Inhibition of K217N/K218D (K62N-K63D)-APC by PCI was 3-5 times more efficient in the absence of heparin and two times less efficient in the presence of heparin. These results indicate that residues E215 (E60a), S216 (S61) and S336 (S173) influence the interaction of APC with heparin and/or AAT and residues K217 (K62) and K218 (K63) are important for heparin stimulation of APC inhibition by PCI $(104,116,117)$. 


\section{Active site mutants}

A much studied APC variant $(17,71,73,118)$ is S360A (S195A)-APC, wherein the active site serine S360 (S195) is mutated to alanine. Consequently, this APC is unable to proteolyse substrates (71). Remarkably, in plasma-based assays, S360A (S195A)-APC still has $15-25 \%$ of the anticoagulant activity of wt-APC. S360A (S195A)-APC had a dose-dependent effect on the activity of the purified prothrombinase complex (17, 119), in particular at low prothrombin and FXa concentrations, since the Kd's for the interactions between FVaFXa and FVaS360A (S195A)-APC are similar (17). Inhibition of FVa by S360A (S195A)-APC is independent of protein S. S360A (S195A)-APC is able to inhibit FVIIIa, but 1-2 orders of magnitude higher concentrations were required compared to FVa inactivation, which illustrates that APC may be of lesser importance for the inactivation of FVIII activity. Remarkably, S360A (S195A)-APC did not show any anticoagulant activity in a $\mathrm{FV}_{\text {Leiden }}$ plasma $(17,71)$. It was shown that the affinity of S360A (S195A)-APC for $\mathrm{FV}_{\text {Leiden }}$ is 150-fold lower than the affinity for wt-FV and that the availability of an R506 residue in FVa is critical for the non-enzymatic binding interaction of FVa and S360A (S195A)-APC (17, 71). S360A (S195A)-APC is devoid of cytoprotective effects $(70,73)$ and can not cleave PAR-1 (118). S360A (S195A)-APC does not form complexes with serpins and therefore has a longer half-life in plasma compared to wt-APC (71). Collectively these data indicate that for the expression of anticoagulant activity, binding of APC, under certain conditions, is sufficient, however a functional active site is required for proteolysis of APC substrates such as coagulation cofactors, inhibitors and PAR-receptors.

\section{Other catalytic domain mutants}

An interesting engineered APC variant is C222-C237 (C67-C82)-APC in which a disulfide bond was introduced between two antiparallel $\beta$-structures consisting of residues that form the $\mathrm{Ca}^{2+}$-binding loop of $\operatorname{APC}(70,120)$. Binding of $\mathrm{Ca}^{2+}$ to this loop results in overall stabilisation of the catalytic domain. As a result, PC changes to a thrombin-(hyper) activatable conformation in which recognition by the thrombinTM complex and the subsequent activation of PC are enhanced $(70,120)$. Due to the engineered disulfide bond, C222-C237 (C67-C82)-PC is trapped in the thrombin(hyper) activatable conformation, even in the absence of $\mathrm{Ca}^{2+}$, resulting in a 80-fold increased activation rate in the absence of $\mathrm{Ca}^{2+}$ and TM. The amidolytic activity of the mutant and its inactivation by PCI were unaffected (120). C222-C237 (C67-C82)-APC has strongly reduced anticoagulant activity in both purified and plasma-based clotting assays (70). Two-fold higher concentrations of C222-C237 (C67-C82)-APC are required to reach cytoprotection that is comparable to wt-PC, likely due to lower EPCR affinity. These results show that occupation of the $\mathrm{Ca}^{2+}$-binding loop is not necessary for the expression of cellular activities. It is not known why the introduction of a disulfide 
bond abolishes the anticoagulant activity of APC, but a changed conformation of the APC catalytic domain may likely impair the interaction of C222-C237 (C67-C82)-APC with FVa and FVIIIa (70).

Two glutamic acid residues at position 330 (167) and 333 (170), located in helix 325333 (helix 162-170) are not conserved in homologous regions of other vitamin-Kdependent proteins. Replacement of these residues by alanine yielded variants that were indistinguishable from wt-APC except for their cytoprotective properties (118). E330A (E167A)-APC and E333A (E170A)-APC show normal interactions with EPCR, but display dramatically impaired cleavage of PAR-1. Thus, both E330 (E167) and E333 (E170) are required for the interaction of APC with PAR-1 and mutation of these residues results in a loss of cytoprotective activity (118).

To investigate the role of N-linked glycosylation on APC functions, four recombinant PC variants in which the asparagines at 97, 248 (93), 313 (150) and 329 (166) were mutated to glutamine, were generated $(5,121)$. Elimination of the latter three glycosylation sites increased activation by the thrombin-TM complex and improved anticoagulant activity in both a modified APTT assay and in a thrombin generation assay $(5,121)$. Notably, the N329Q (N166Q) mutation influenced the cytoprotective function of APC. In an endothelial barrier permeability assay, N329Q (N166Q)-APC was up to six-fold more efficient than wt-APC and in staurosporine-induced endothelial cell apoptosis, N329Q (N166Q)-APC completely inhibited apoptosis at a 30-fold lower concentration than wt APC (121). Possibly the absence of a N-linked glycan chain at N329 enhances the access of residues E330 and E333 to PAR-1 and EPCR-dependent PAR-1 activation by APC is up-regulated, resulting in increased cytoprotection (121).

E357 (E192) is involved in binding of macromolecular substrates. Engineered E357Q (E192Q)-APC showed two- to three-fold improved FVa inactivation, but slightly reduced anticoagulant activity in plasma compared to wt-APC, likely due to an observed 280-fold faster inhibition by AAT (122). In contrast to wt-APC, this variant is effectively inactivated by pancreatic trypsin inhibitor, TFPI and AAT in both the presence and absence of heparin. These findings hint at an evolved function of E357 (E192) in order to prevent fast inhibition by serpins, at the cost of a reduced FVa inactivation (122). 


\section{Conclusion}

APC is a protease with both anticoagulant and cytoprotective functions. Several areas on the surface of (A)PC are involved in the many interactions between (A)PC and its ligands (Figure 6). Mutagenesis studies proved the importance of a number of residues in the Gla-domain for the anticoagulant function of PC. The correct $\mathrm{Ca}^{2+}$-dependent conformation of this domain is essential for its binding to PL membranes and EPCR. Several studies have been performed to study the mechanism of PL binding for Gladomains in general and for PC in particular. Detailed structure-function data have provided a rational basis for protein engineering of $\mathrm{PC}$, which has resulted in variants of PC with improved membrane binding, which may prove effective in the pharmacological use of PC $(76,77,86)$. Residues in 191-loop (37-loop) the $\mathrm{Ca}^{2+}$-binding loop and the autolysis loop of the protease domain are important for electrostatic interactions between APC and FVa, the main physiological APC substrate in coagulation. Residues interacting with protein $\mathrm{S}$ are situated in the helical aromatic segment (residue 38), EGF domain (residue 71), linking peptide (residue 149) and autolysis loop. For the PC activation by thrombin and the thrombin-TM-complex, residues in close proximity to residue 169 are important, as well as residues in the Gla- domain (residue 16 and 26), 191-loop (37-loop), 214-loop (60-loop), the $\mathrm{Ca}^{2+}$-binding loop and the autolysis loop. The interaction of PC with heparin, PCI and AAT is mediated by residues in 191-loop (37-loop), 214-loop (60-loop) and residue 336 (173). The autolysis loop and residues 357 (192) and 360 (195) are important for the interaction of PC with serpins. Mutagenesis studies have indicated that the more recently discovered cytoprotective functions of APC can be linked to residues 149, 329 (166), 330 (167), 333 (170) and 360 (195), which are essential for cytoprotection. The effects of a number of other APC variants, which have been characterised for their anticoagulant properties, have not been investigated so far in the context of their cytoprotective properties and this research is currently ongoing. Considering the interest from both the academia and the pharmaceutical industry in PC, it is desirable and likely that a completed structure- function description of the cytoprotective properties of APC will become available in the next years. This will aid in the engineering of a yet safer variant of APC devoid of increased bleeding risks, for use in cell-protection in a number of relevant clinical conditions of which severe sepsis appears the most prominent.

\section{Acknowledgements}

This work was supported by a Special Project Grant from the Bayer Haemophilia Awards Program (to G. A. F. N.) and by the Cardiovascular Research Institute Maastricht (to G. A. F. N.) 


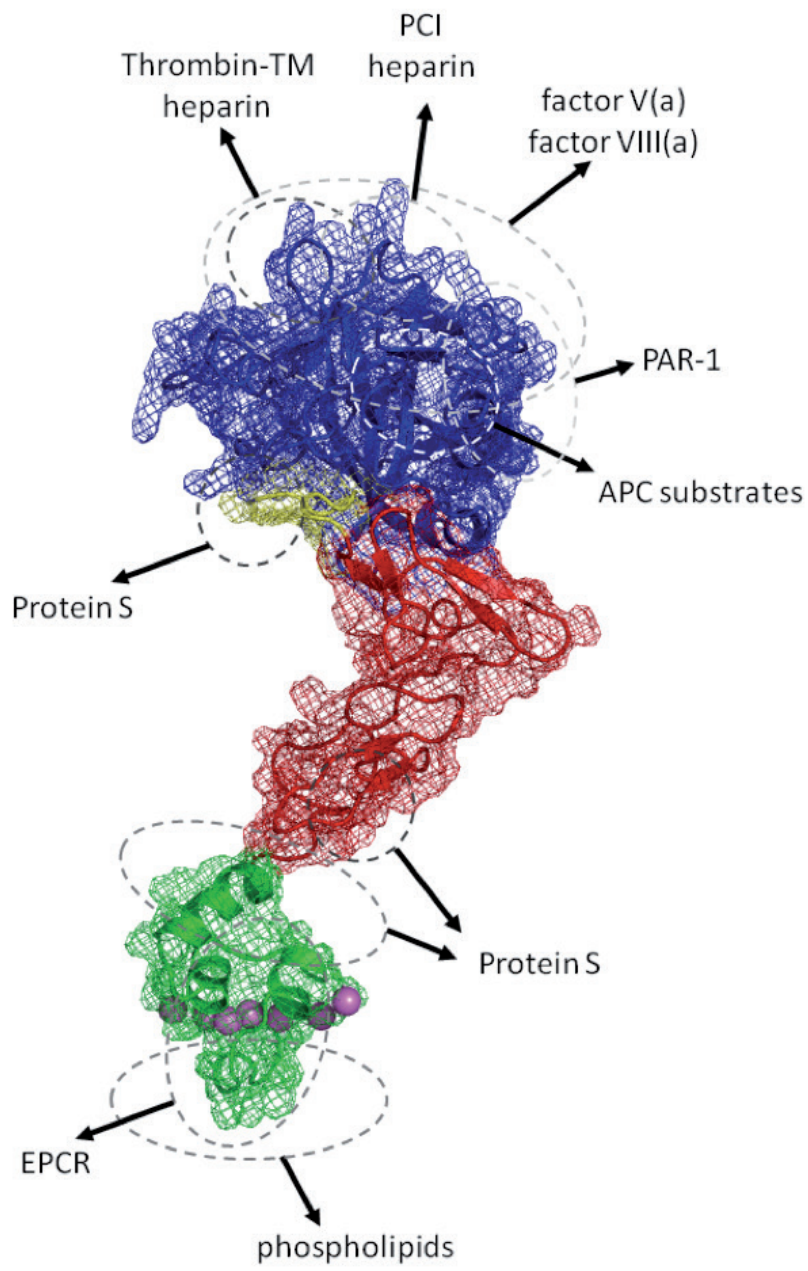

\section{Figure 6: Mapping of APC interactions on its overall 3D structure}

The mosaic protein architecture of APC is shown. The different domains are shown in their ribbon structure with the Vanderwaals protein surface overlaid as a mesh representation to better illustrate the volume and shape of the protease. From N-terminus to C-terminus are shown: green, the Gla-domain, residues $1-37$, residue numbers refer to the mature protein) including a helical aromatic segment, residues 38-45; red, EGF-1 and EGF-2 domains, residues 46-92 and 93-136 respectively; yellow, a linking peptide (residues 137-157); blue, the trypsin-like serine protease domain, residues 170-419 (16-254). Indicated are interactions in the exosites and active site. Exosite regions have been described that facilitate the interactions between APC and FV(a)/ FVIII(a), thrombin-TM complex, PCI, heparin, PAR-1, protein S, EPCR and PL. Likewise, the interaction of the APC active site with APC substrates is pharmacological pharmacological use of PC $(76,77,86)$. Residues in 191-loop (37-loop) the $\mathrm{Ca}^{2+}$-binding loop necessary for proteolysis. The activation peptide, residues 158-169, is not visible after its removal due to activation of PC, interactions to this region (as with thrombin or the thrombin-TM complex) are not shown. 


\section{References}

1. Griffin JH, Fernandez JA, Gale AJ, et al. Activated protein C. J Thromb Haemost 2007; 5: 73-80.

2. Stearns-Kurosawa D, Kurosawa S, Mollica J, et al. The endothelial cell protein C receptor augments protein C activation by the thrombin-thrombomodulin complex. PNAS 1996; 96: 10212-10216.

3. Mather T, Oganessyan V, Hof P, et al. The $2.8 \AA$ crystal structure of Gla-domainless activated protein C. EMBO J 1996; 15: 6822-6831.

4. UniProtKB/Swiss-Prot, PROC_HUMAN, accession number P04070. 2011.

5. Grinnell B, Walls J, Gerlitz B. Glycosylation of human protein C affects its secretion, processing, functional activities, and activation by thrombin. J Biol Chem 1991; 266: 9778-9785.

6. Scully $\mathrm{M}$, Toh $\mathrm{C}$, Hoogendoorn $\mathrm{H}$, et al. Activation of protein $\mathrm{C}$ and its distribution between its inhibitors, protein $\mathrm{C}$ inhibitor, alpha-1-antitrypsin and alpha-2-macroglobulin in patients with disseminated intravascular coagulation Thromb Haemost 1993; 69: 448-453.

7. Heeb M, Gruber A, Griffin J. Identification of divalent metal ion-dependent inhibition of activated protein $\mathrm{C}$ by alpha-2-macroglobulin and alpha-2-antiplasmin in blood and comparisons to inhibition of factor Xa, thrombin and plasmin. J Biol Chem 1991; 266: 17606-17612.

8. Zhu T, Ubhayasekera W, Nickolaus N, et al. Zinc ions bind to and inhibit activated protein C. Thromb Haemost 2010; 104: 544-553.

9. Mammen EF, Thomas WR, Seegers WH. Activation of purified prothrombin to autoprothrombin I or autoprothrombin II (platelet cofactor II or autoprothrombin II-A). Throm Diath Haemorrh 1976; 5: 218-249.

10. Stenflo J. A new vitamin K-dependent protein. J Biol Chem 1976; 251: 355-363.

11. Kalafatis M, Rand M, Mann K. The mechanism of inactivation of human factor $V$ and human factor Va by activated protein C. J Biol Chem 1994; 269: 31869-31880.

12. Nicolaes G, Tans G, Thomassen M, et al. Peptide bond cleavages and loss of functional activity during inactivation of factor Va and factor VaR506Q by activated protein C. J Biol Chem 1995; 270: 21158-21166.

13. Rosing J, Hoekema L, Nicolaes G, et al. Effects of protein S and factor Xa on peptide bond cleavages during inactivation of factor Va and factor VaR506Q by activated protein C. J Biol Chem 1995; 270: 27852-27858.

14. Yegneswaran S, Wood G, Esmon C, et al. Protein S alters the active site location of activated protein C above the membrane surface. A fluorescence resonance energy transfer study. J Biol Chem 1997; 272: 25013-25021.

15. Yegneswaran S, Smirnov M, Safa O, et al. Relocating the active site of activated protein C eliminates the need for its protein S cofactor. A fluorescence resonance energy transfer study. J Biol Chem 1999; 274: 5462-5468.

16. Segers K, Dahlbäck B, Rosing J, et al. Identification of surface epitopes of human coagulation factor Va that are important for interaction with activated protein C and heparin. J Biol Chem 2008; 283: $22573-22581$.

17. Nicolaes GAF, Bock PE, Segers K, et al. Inhibition of thrombin formation by active site mutated (S360A) activated protein C. J Biol Chem 2010; 285: 22890-22900.

18. Kim P, Nesheim M. Down regulation of prothrombinase by activated protein $\mathrm{C}$ during prothrombin activation. Thromb Haemost 2010; 104: 61-70. 
19. Fay P, Smudzin T, Walker F. Activated protein C-catalyzed inactivation of human factor VIII and factor VIIIa. Identification of cleavage sites and correlation of proteolysis with cofactor activity. J Biol Chem 1991; 266: 20139-20145.

20. Amano K, Michnick D, Moussalli M, et al. Mutation at either Arg336 or Arg562 in factor VIII is insufficient for complete resistance to activated protein C (APC)-mediated inactivation: implications for the APC resistance test. Thromb Haemost 1998; 79: 557-563.

21. Nyberg P, Dahlbäck B, Garcia de Frutos P. The SHBG-like region of protein S is crucial for factor V-dependent APC-cofactor function. FEBS Lett 1998; 433:28-32.

22. Thorelli E, Kaufman RJ, Dahlbäck B. Cleavage of factor V at Arg 506 by activated protein C and the expression of anticoagulant activity of factor V. Blood 1999; 93:2552-2558.

23. Thorelli E, Kaufman R, Dahlbäck B. The C-terminal region of the factor V B-domain is crucial for the anticoagulant activity of factor V. J Biol Chem 1998; 273:16140-16145.

24. Lollar P, Parker C. pH-dependent denaturation of thrombin-activated porcine factor III. J Biol Chem 1990; 265: 1688-1692.

25. Nicolaes G, Dahlbäck B. Activated protein C resistance (FV(Leiden)) and thrombosis:factor V mutations causing hypercoagulable states. Hematol Oncol Clin North Am 2003; 17: 37-61.

26. Nicolaes GAF, Dahlbäck B. Congenital and acquired activated protein C resistance. Semin Vasc Med 2003; 3: 33-46.

27. Dahlbäck B, Villoutreix BO. Regulation of blood coagulation by the protein C anticoagulant pathway. Novel insights into structure-function relationships and molecular recognition. Vasc Biol 2005; 25: 1311-1320.

28. Broekmans AW, Veltkamp JJ, Bertina RM. Congenital protein C deficiency and venous thromboembolism. A study of three Dutch families. N Engl J Med 1983; 309:340-344.

29. Griffin JH, Evatt B, Zimmerman TS, et al. Deficiency of protein C in congenital thrombotic disease. J Clin Invest 1981; 68: 1370-1373.

30. Zorio E, Navarro S, Medina P, et al. Circulating activated protein $C$ is reduced in young survivors of myocardial infarction and inversely correlates with the severity of coronary lesions. J Thromb Haemost 2006; 4: 1530-1536.

31. España F, Vayá A, Mira Y, et al. Low level of circulating activated protein C is a risk factor for venous thromboembolism. Thromb Haemost 2001; 86: 1368-1373.

32. Marlar R, Neumann A. Neonatal purpura fulminans due to homozygous protein $\mathrm{C}$ or protein $\mathrm{S}$ deficiencies. Semin Thromb Hemost 1990; 16: 299-309.

33. Marlar RA, Montgomery RR, Broekmans AW. Diagnosis and treatment of homozygous protein C deficiency. Report of the Working Party on Homozygous Protein C Deficiency of the Subcommittee on Protein C and Protein S, International Committee on Thrombosis and Haemostasis. J Pediatr 1989; 114:528-534.

34. Seligsohn U, Berger A, Abend M, et al. Homozygous protein C deficiency manifested by massive venous thrombosis in the newborn. N Engl J Med 1984; 310:559-562.

35. Branson HE, Katz J, Marble R, et al. Inherited protein C deficiency in a neonate. Lancet 1983; 2: $1165-1168$.

36. Jackson C, Xue M, Thompson P, et al. Activated protein C prevents inflammation yet stimulates angiogenesis to promote cutaneous wound healing. Wound Repair Regen 2005; 13: 284-294.

37. Isobe $\mathrm{H}$, Okajima $\mathrm{K}$, Uchiba $\mathrm{M}$, et al. Activated protein $\mathrm{C}$ prevents endotoxin-induced hypotension in rats by inhibiting excessive production of nitric oxide. Circulation 2001; 104: 1171-1175.

38. Taylor $\mathrm{Fj}$, Chang A, Esmon $\mathrm{C}$, et al. Protein $\mathrm{C}$ prevents the coagulopathic and lethal effects of Escherichia coli infusion in the baboon. J Clin Invest 1987; 79: 918-925. 
39. Iba T, Kidokoro A, Fukunaga M, et al. Activated protein $C$ improves the visceral microcirculation by attenuating the leukocyte-endothelial interaction in a rat lipopolysaccharide model. Crit Care Med 2005; 33: 368-372.

40. Xue M, Thompson P, Sambrook P, et al. Activated protein C stimulates expression of angiogenic factors in human skin cells, angiogenesis in the chick embryo and cutaneous wound healing in rodents. Clin Hemorheol Microcirc 2006; 34:153-161.

41. Zlokovic B, Zhang C, Liu D, et al. Functional recovery after embolic stroke in rodents by activated protein C. Ann Neurol 2005; 58: 474-477.

42. Liu D, Cheng T, Guo H, et al. Tissue plasminogen activator neurovascular toxicity is controlled by activated protein C. Nat Med 2004; 10: 1479-1483.

43. Cheng T, Petraglia A, Li Z, et al. Activated protein C inhibits tissue plasminogen activator-induced brain hemorrhage. Nat Med 2006; 12: 1278-1285.

44. Murakami K, Okajima K, Uchiba M, et al. Activated protein C attenuates endotoxin-induced pulmonary vascular injury by inhibiting activated leukocytes in rats. Blood 1996; 87: 642-647.

45. Abraham E, Reinhart K, Opal S, et al. Efficacy and safety of tifacogin (recombinant tissue factor pathway inhibitor) in severe sepsis: a randomized controlled trial. J Am Med Assoc 2003; 290: 238247.

46. Warren BL, Eid A, Singer P, et al. Caring for the critically ill patient. High-dose antithrombin III in severe sepsis: a randomized controlled trial. J Am Med Assoc 2001; 286: 1869-1878.

47. Bernard GR, Vincent JL, Laterre PF, et al. Efficacy and safety of recombinant human activated protein C for severe sepsis. N Engl J Med 2001; 344: 699-709.

48. Martí-Carvajal A, Solà I, Lathyris D, et al. Human recombinant activated protein C for severe sepsis. Cochrane Database Syst Rev 2011; CD004388.

49. Coughlin S. Thrombin signalling and protease-activated receptors. Nature 2000;407: 258-264.

50. Ossovskaya VS, Bunnet NW. Protease-activated receptors: contribution to physiology and disease. Physiol Rev 2004; 84: 579-621.

51. Nakamura M, Gabazza E, Imoto I, et al. Anti-inflammatory effect of activated protein C in gastric epithelial cells. J Thromb Haemost 2005; 3: 2721-2729.

52. Bae J, Yang L, Rezaie A. Receptors of the protein $\mathrm{C}$ activation and activated protein $\mathrm{C}$ signaling pathways are colocalized in lipid rafts of endothelial cells. Proc Natl Acad Sci USA 2007; 104: 28672872.

53. Gorbacheva L, Davidova O, Sokolova E, et al. Endothelial protein C receptor is expressed in rat cortical and hippocampal neurons and is necessary for protective effect of activated protein $\mathrm{C}$ at glutamate excitotoxicity. J Neurochem 2009; 111:967-975.

54. Gillibert-Duplantier J, Rullier A, Neaud V, et al. Liver myofibroblasts activate protein C and respond to activated protein C. World J Gastroenterol 2010; 16:210-216.

55. Mosnier LO, Zlokovic BV, Griffin JH. The cytoprotective protein C pathway.Blood 2007; 109: 31613172.

56. Loubele ST, Spek CA, Leenders P, et al. Activated protein C protects against myocardial ischemia/ reperfusion injury via inhibition of apoptosis and inflammation. Arterioscler Thromb Vasc Biol 2009; 29: 1087-1092.

57. Fernandez JA, Xu X, Liu D, et al. Recombinant murine activated protein C is neuroprotective in a murine ischemic stroke model. Blood Cells Mol Dis 2003; 30:271-276.

58. Yang X, Banerjee Y, Fernandez J, et al. Activated protein C ligation of ApoER2 (LRP8) causes Dab1dependent signaling in U937 cells. PNAS 2009; 106:274-279. 
59. Cao C, Gao Y, Li Y, et al. The efficacy of activated protein $\mathrm{C}$ in murine endotoxemia is dependent on integrin CD11b. J Clin Invest 2010; 120: 1971-1980.

60. Xue M, Chow S, Dervish S, et al. Activated protein C enhances human keratinocyte barrier integrity via sequential activation of epidermal growth factor receptor and Tie2. J Biol Chem 2011; 286: 67426750.

61. Joyce DE, Gelbert L, Ciaccia A, et al. Gene expression profile of antithrombotic protein C defines new mechanisms modulating inflammation and apoptosis. J Biol Chem 2001; 276: 11199-11203.

62. Kerschen E, Hernandez I, Zogg M, et al. Activated protein C targets CD8+ dendritic cells to reduce the mortality of endotoxemia in mice. J Clin Invest 2010;120:3167-3178.

63. Mosnier L, Griffin J. Inhibition of staurosporine-induced apoptosis of endothelial cells by activated protein $\mathrm{C}$ requires protease-activated receptor-1 and endothelial cell protein $\mathrm{C}$ receptor. Biochem $\mathrm{J}$ 2003; 373: 65-70.

64. Cheng T, Dong L, Griffin J, et al. Activated protein C blocks p53-mediated apoptosis in ischemic human brain endothelium and is neuroprotective. Nat Med 2003; 9: 338-342.

65. Guo H, Liu D, Gelbard H, et al. Activated protein $\mathrm{C}$ prevents neuronal apoptosis via protease activated receptors 1 and 3. Neuron 2004; 41: 563-572.

66. Riewald M, Petrovan R, Donner A, et al. Activation of endothelial cell protease activated receptor 1 by the protein C pathway. Science 2002; 296: 1880-1882.

67. Gateau-Roesch O, Argaud L, Ovize M. Mitochondrial permeability transition pore and postconditioning. Cardiovasc Res 2006; 70: 264-273.

68. Rodrigues S, Granger D. Role of blood cells in ischaemia-reperfusion induced endothelial barrier failure. Cardiovasc Res 2010; 87: 291-299.

69. Minhas N, Xue M, Fukudome K, et al. Activated protein C utilizes the angiopoietin/Tie2 axis to promote endothelial barrier function. FASEB J 2009; 24: 873-881.

70. Bae JS, Yang L, Manithody C, et al. Engineering a disulfide bond to stabilize the calcium-binding loop of activated protein $\mathrm{C}$ eliminates its anticoagulant but not its protective signaling properties. J Biol Chem 2007; 282: 9251-9259.

71. Gale AJ, Sun X, Heeb MJ, et al. Nonenzymatic anticoagulant activity of the mutant serine protease Ser360Ala-activated protein C mediated by factor Va. Protein science 1997; 6: 132-140.

72. Kerschen EJ, Fernandez JA, Cooley BC, et al. Endotoxemia and sepsis mortality reduction by nonanticoagulant - activated protein C. J Exp Med 2007; 204:2439-2448.

73. Mosnier LO, Gale AJ, Yegneswaran S, et al. Activated protein C variants with normal cytoprotective but reduced anticoagulant activity. Blood 2004; 104:1740-1744.

74. Mosnier LO, Yang XV, Griffin JH. Activated protein C mutant with minimal anticoagulant activity, normal cytoprotective activity, and preservation of thrombin activable fibrinolysis inhibitor-dependent cytoprotective functions. J Biol Chem 2007; 282: 33022-33033.

75. Mosnier LO, Zampolli A, Kerschen EJ, et al. Hyperantithrombotic, noncytoprotective Glu149Alaactivated protein C mutant. Blood 2009; 113: 5970-5978.

76. Sun Y, Tran S, Norstrøm E, et al. Enhanced rate of cleavage at Arg-306 and Arg-506 in coagulation factor Va by gla domain-mutated human-activated protein C. J Biol Chem 2004; 279: 47528-47535.

77. Sun YH, Shen L, Dahlbäck B. Gla domain-mutated human protein C exhibiting enhanced anticoagulant activity and increased phospholipid binding. Blood 2003; 101: 2277-2284.

78. Zhang L, Castellino F. The binding energy of human coagulation protein $\mathrm{C}$ to acidic phospholipid vesicles contains a major contribution from leucine 5 in the y-carboxyglutamic acid domain. J Biol Chem 1994; 269: 3590-3595. 
79. Preston R, Ajzner E, Razzari C, et al. Multifunctional specificity of the protein C/activated protein C Gla domain. J Biol Chem 2006; 281: 28850-2887.

80. Christiansen W, Jalbert L, Robertson R, et al. Hydrophobic amino acid residues of human anticoagulation protein $\mathrm{C}$ that contribute to its functional binding to phospholipid Vesicles. Biochemistry 1995; 34 : 10376-10382.

81. McDonald J, Shah A, Schwalbe R, et al. Comparison of naturally occurring vitamin K-dependent proteins: correlation of amino acid sequences and membrane binding properties suggests a membrane contact site. Biochemistry 1997; 36:5120-5127.

82. Shen L, Shah A, Dahlbäck B, et al. Enhancing the activity of protein C by mutagenesis to improve the membrane-binding site: studies related to Proline-10. Biochemistry 1997; 36: 16025-16031.

83. Zhang L, Jhingan A, Castellino F. Role of individual ?-carboxyglutamic acid residues of activated human protein $C$ in defining its in vitro anticoagulant activity. Blood 1992; 80: 942-952.

84. Oganesyan V, Oganesyan N, Terzyan S, et al. The crystal structure of the endothelial protein C receptor and a bound phospholipid. J Biol Chem 2002; 277:24851-24854.

85. Qureshi S, Yang L, Manithody C, et al. Functional properties and active-site topographies of factor X Gla- and prothrombin Gla-domain chimeras of activated protein C. Biochim Biophys Acta 2008; 1780: 1080-1086.

86. Preston R, Villegas-Mendez A, Sun Y, et al. Selective modulation of protein C affinity for EPCR and phospholipids by Gla domain mutation. FEBS J 2005; 272:97-108.

87. Zhang L, Castellino F. A y-Carboxyglutamic Acid (y) Variant (y6D, y7D) of Human Activated Protein C Displays Greatly Reduced Activity as an Anticoagulant.Biochemistry 1990; 29: 10828-10834.

88. Zhang L, Castellino F. Role of the Hexapeptide Disulfide Loop Present in the y-Carboxyglutamic Acid Domain of Human Protein C in Its Activation Properties and in the in Vitro Anticoagulant Activity of Activated Protein C. Biochemistry 1991; 30: 6696-6704.

89. Gaussem P, Gandrille S, Duchemin J, et al. Influence of six mutations of the protein C gene on the Gla domain conformation and calcium affinity. Thromb Haemost 1994; 71: 748-754.

90. Gandrille S, Alhenc-Gelas M, Gaussem P, et al. Five novel mutations located in exons III and IX of the protein $\mathrm{C}$ gene in patients presenting with defective protein C anticoagulant activity. Blood 1993; 82: $159-168$.

91. Bovill E, Tomczak J, Grant B, et al. Protein CVermont: symptomatic type II protein C deficiency associated with two GLA domain mutations. Blood 1992; 79:1456-1465.

92. Thariath A, Castellino F. Highly conserved residue arginine-15 is required for the $\mathrm{Ca}^{2+}$-dependent properties of the gamma-carboxyglutamic acid domain of human anticoagulation protein $\mathrm{C}$ and activated protein C. Biochem J 1997; 322: 309-315.

93. Iijima K, Fukuda C, Nakamura K, et al. A new hereditary abnormal protein C (protein C Yonago) with a dysfunctional Gla-domain. Thromb Res 1991; 63:249-257.

94. Mimuro J, Muramatsu S, Kaneko M, et al. An abnormal protein C (protein C Yonago) with an amino acid substitution of Gly for Arg-15 caused by a single base mutation of $\mathrm{C}$ to $\mathrm{G}$ in codon 57 (CGG-$>$ GGG). Deteriorated calcium-dependent conformation of the gamma-carboxyglutamic acid domain relevant to a thrombotic tendency. Int J Hematol 1993; 57: 9-14.

95. Harmon S, Preston RJS, Ainle FN, et al. Dissociation of activated protein C functions by elimination of protein S cofactor enhancement. J Biol Chem 2008; 283:30531-30539.

96. Rezaie A, Esmon C. The function of calcium in protein $\mathrm{C}$ activation by thrombin and the thrombinthrombomodulin complex can be distinguished by mutational analysis of protein $\mathrm{C}$ derivatives. J Biol Chem 1992; 267: 26104-26109. 
97. Ohlin A, Landes G, Bourdon P, et al. Beta-hydroxyaspartic acid in the first epidermal growth factorlike domain of protein C. Its role in $\mathrm{Ca}^{2+}$ binding and biological activity. J Biol Chem 1988; 263 : 19240-19248.

98. Ohlin A, Björk I, Stenflo J. Proteolytic formation and properties of a fragment of protein C containing the gamma-carboxyglutamic acid rich domain and the EGF-like region. Biochemistry 1990; 29: 644 651.

99. Rezaie A, Esmon C. Proline at the P2 position in protein C is important for calcium-mediated regulation of protein C activation and secretion. Blood 1994; 83:2526-2531.

100. Yang L, Prasad S, Di Cera E, et al. The conformation of the activation peptide of protein C is influenced by $\mathrm{Ca}^{2+}$ and $\mathrm{Na}+$ binding. J Biol Chem 2004; 279:38519-38524.

101. Grinnell B, Gerlitz B, Berg D. Identification of a region in protein C involved in thrombomodulinstimulated activation by thrombin: potential repulsion at anion-binding site I in thrombin. Biochem J 1994; 303: 929-933.

102. Friedrich U, Nicolaes G, Villoutreix B, et al. Secondary substrate-binding exosite in the serine protease domain of activated protein C important for cleavage at Arg-506 but not at Arg-306 in factor Va. J Biol Chem 2001; 276: 2305-2308.

103. Gale A, Tsavaler A, Griffin J. Molecular characterization of an extended binding site for coagulation factor Va in the positive exosite of activated protein C. J Biol Chem 2002; 277: 28836-28840.

104. Friedrich U, Blom A, Dahlbäck B, et al. Structural and energetic characteristics of the heparin-binding site in antithrombotic protein C. J Biol Chem 2001; 276:24122-24128.

105. Gerlitz B, Grinnell B. Mutation of protease domain residues Lys37-39 in human protein C inhibits activation by the thrombomodulin-thrombin complex without affecting activation by free thrombin. J Biol Chem 1996; 271: 22285-22288.

106. Glasscock L, Gerlitz B, Coopera S, et al. Basic residues in the 37-loop of activated protein C modulate inhibition by protein C inhibitor but not by a1-antitrypsin. Biochim Biophys Acta 2003; 1649: 106117.

107. Manithody C, Fay P, Rezaie A. Exosite-dependent regulation of factor VIIIa by activated protein C. Blood 2003; 101: 4802-4807.

108. Alhenc-Gelas M, Gandrille S, Aubry M, et al. Thirty-three novel mutations in the protein C gene. French INSERM network on molecular abnormalities responsible for protein $\mathrm{C}$ and protein $\mathrm{S}$. Thromb Haemost 2000; 83: 86-92.

109. Vincenot A, Gaussem P, Pittet J, et al. Amino acids 225-235** of the protein C serine-protease domain are important for the interaction with the thrombinthrombomodulin complex. FEBS lett 1995; 367: 153-157.

110. Fuentes-Prior P, Iwanaga Y, Huber R, et al. Structural basis for the anticoagulant activity of the thrombin-thrombomodulin complex. Nature 2000; 404: 518-525.

111. Fisher C, Greengard J, Griffin J. Models of the serine protease domain of the human antithrombotic plasma factor activated protein C and its zymogen. Protein Sci 1994; 3: 588-599.

112. Shen L, Villoutreix B, Dahlbäck B. Interspecies loop grafting in the protease domain of human protein C yielding enhanced catalytic and anticoagulant activity. Thromb Haemost 1999; 82: 1078-1087.

113. Yang L, Manithody C, Rezaie A. The functional significance of the autolysis loop in protein C and activated protein C. Thromb Haemost 2005; 94: 60-68. 114. Cramer T, Gale A. Function of the activated protein C (APC) autolysis loop in activated FVIII inactivation. Br J Haematol 2011; 153 : 644-654.

115. Gale A, Heeb M, Griffin J. The autolysis loop of activated protein C interacts with factor Va and differentiates between the Arg506 and Arg306 cleavage sites. Blood 2000; 96: 585-593. 
116. Shen L, Dahlbäck B, Villoutreix B. Tracking structural features leading to resistance of activated protein C to a1-antitrypsin. Biochemistry 2000; 39:2853-2860.

117. Shen L, Villoutreix B, Dahlbäck B. Involvement of Lys 62[217] and Lys 63[218] of Human Anticoagulant Protein C in Heparin Stimulation of Inhibition by the Protein C Inhibitor. Thromb Haemost 1999; 82: 72-79.

118. Yang L, Bae JS, Manithody C, et al. Identification of a Specific Exosite on Activated Protein C for Interaction with Protease-activated Receptor 1. J Biol Chem 2007; 282: 25493-25500.

119. Yegneswaran S, Kojima Y, Nguyen P, et al. Factor Va residues 311-325 represent an activated protein C binding region. J Biol Chem 2007; 282: 28353-28361.

120. Yang L, Manithody C, Rezaie A. Activation of protein C by the thrombin-thrombomodulin complex: Cooperative roles of Arg-35 of thrombin and Arg-67 of protein C. PNAS 2006; 103: 879-884.

121. Ní Ainle F, O’Donnell J, Johnson J, et al. Activated protein C N-linked glycans modulate cytoprotective signaling function on endothelial cells. J Biol Chem 2011; 286: 1323-1330.

122. Rezaie A, Esmon C. Conversion of glutamic acid 192 to glutamine in activated protein $C$ changes the substrate specificity and increases reactivity toward macromolecular inhibitors. J Biol Chem 1993; 268: 19943-19948.

123. Soriano-Garcia M, Padmanabhan $\mathrm{K}$, de Vos A, et al. The $\mathrm{Ca}^{2+}$ ion and membrane binding structure of the Gla domain of Ca-prothrombin fragment 1. Biochemistry 1992; 31: 2554-2566. 



\section{Chapter 3}

\section{Identification of novel small molecule inhibitors of activated protein $\mathrm{C}$}

Olivier Sperandio ${ }^{1 *}$, Karin C.A.A. Wildhagen ${ }^{2 *}$, Roy Schrijver ${ }^{2}$, Simone Wielders ${ }^{2}$, Bruno O. Villoutreix ${ }^{1 *}$, Gerry A.F. Nicolaes ${ }^{2 *}$

*These authors contributed equally to this work.

1. INSERM U973, University Paris Diderot, F-75205 Paris, France

2. Department of Biochemistry, Cardiovascular Research Institute Maastricht, Maastricht University, The Netherlands 


\section{Abstract}

Introduction: Activated protein C (APC) is the central enzyme of the anticoagulant protein $\mathrm{C}$ pathway. Low concentrations of APC circulate in plasma and are believed to contribute to the maintenance of anormal haemostatic balance.

Materials and Methods: We have used a structure-based virtual screening approach to discover small drug-like molecules that inhibit the interaction between APC and its substrate FVa through inhibition of a predominant APC exosite,known to be involved in FVa substrate binding. We have combined in silico selection with functional screening and direct binding analysis to identify novel molecules and to ascertain and characterize the inhibition of the interaction between APC and FVa.

Results: We have identified a number of novel molecules that bind to APC and protein C with $\mathrm{Kd}$ values in the range of $10^{-3}-10^{-5} \mathrm{M}$. Inhibition by these molecules is incomplete, which most likely reflects the extended surface that is involved in the interaction between APC and its substrates. Direct binding of hit molecules to variant APC molecules that were mutated in the targeted binding site revealed that several of the molecules presented a 100-500 fold lower affinity for the variant molecule, suggesting that these molecules indeed bind the exosite of APC.

Conclusions: The protein-protein interaction inhibitors discovered here, could function as starting molecules for further development of small molecules with anti-APC properties. Such molecules may be of clinical interest, in particular in individuals where thrombin formation is compromised and the haemostatic balance is tipped towards bleeding tendencies, such as in haemophilia A. 


\section{Introduction}

Activated protein $\mathrm{C}$ (APC) is the central protease of the anticoagulant protein $\mathrm{C}$ pathway and APC, together with its cofactor, protein $\mathrm{S}$, is of vital importance to the regulation of the procoagulant activities of the activated cofactor proteins factor VIIIa and factor $\mathrm{Va}$ (1). Failure to express the anticoagulant functions of APC, such as in protein $\mathrm{C}$ deficiency, protein $\mathrm{S}$ deficiency or in carriership of the $\mathrm{FV}_{\text {Leiden }}$ mutation is associated with a life-long enhancement of the risk of thromboembolic disease $(2,3)$. The severity of such conditions is most dramatically exemplified in patients who are homozygously deficient of protein C. In these individuals life-threatening thromboses of the microvasculature occur already in the neonatal phase, a clinical disease state named 'purpura fulminans'.

APC is the activated form of protein $\mathrm{C}$, a serine protease zymogen that circulates in human blood at an average concentration of $70 \mathrm{nM}$ (4) and it is estimated that picomolar amounts of APC circulate in plasma, in levels proportional to the protein C levels (5). Protein $C$ is a vitamin- $K$ dependent protein which has a mosaic multi-domain structure in which a $\mathrm{N}$-terminal $\boldsymbol{\gamma}$-carboxyglutamic acid (Gla)-domain, is followed by two epidermal growth factor (EGF)-like domains, a linking peptide, a small activation peptide and a trypsin-like serine protease domain in which the active site triad (residues D257, H211 and S360, protein sequence numbering system according to (6)) are present $(4,6)$. Protein $\mathrm{C}$ is activated by the removal of a 12 -amino acid activation peptide, upon which the active serine protease APC is formed. APC then is able to inactivate the coagulation cofactors FVIIIa and FVa through limited proteolysis of these cofactors, which results in a loss of cofactor activity $(7,8)$, a process which is essential for the maintenance of a normal haemostatic balance. More recently, it was shown that APC has important cellular functions and acts as a cytoprotective protein $(9,10)$.

The most common, genetically determined risk factor for increased in vivo generation of thrombin and consequently thrombosis, is APC resistance, which is caused in 95\% of cases by a mutation in the FV gene, giving rise to $\mathrm{FV}_{\text {Leiden, }}$, which harbors a $\mathrm{R} 506 \mathrm{Q}$ mutation at a predominant APC cleavage site in FV. Due to this mutation, FVaLeiden is down-regulated less efficiently than normal FVa $(11,12)$. Moreover, $\mathrm{FV}_{\text {Leiden }}$ has a reduced APC cofactor activity in the proteolytic down-regulation of FVIIIa $(13,14)$. Remarkably, in the case of coinheritance of FVIII-, FIX- or FVII-deficiency and APC resistance, the phenotype of doubly affected carriers is considerably milder than that of factor deficient patients who are not APC resistant. In vitro experiments have verified that in case of e.g. combined FVIII deficiency and $\mathrm{FV}_{\text {Leiden }}$, an increased generation of thrombin is observed in the presence of APC. Thus, the increased procoagulant tendency, due to reduced bioactivity of APC results in an overall beneficial effect in these patients (15-20). These in vitro and in vivo observations suggest that a reduced function/ activity of the protein $\mathrm{C}$ pathway is associated with a relative shift in the haemostatic balance towards a more procoagulant state. In this respect, APC is an attractive target 
for inhibition to provide support of in vivo thrombin generation, as has been put forward by Butenas and colleagues (16) and which was further elaborated on by De Nanteuil and coworkers (21). The latter identified an APC inhibitor with an IC50 of $820 \mathrm{nM}$, which showed reasonably good specificity for APC. The latter inhibitor however contained a hydrophilic benzamidine moiety which may raise hepatotoxicity issues. Both these studies $(16,21)$, used peptidic or peptide-like compounds for inhibition of APC and were aimed at inhibition of the proteolytic activity of APC.

A relatively extensive amount of structure function information is available for FV, FVIII and APC, and amino acid residues have been identified which are of importance to the interaction between APC and its substrates in the anticoagulant response (for a recent review see (1)). Given the importance of APC for cell viability, we decided to specifically target the exosites important for the anticoagulant functions of APC, and in particular those that govern the formation of a productive complex between APC and the cleavage sites at Arg506 and Arg306 in FVa, which are known to be of lesser importance for the cellular APC functions, so as not to interfere with the cytoprotective properties of APC. Thus, the current work was undertaken toinvestigate in model systems the possibility to pharmacologically induce acquired APC resistance in patients who are at risk for bleeding with small molecules. To provide such a proof-of-concept, we aimed at the discovery of inhibitors of protein-protein interaction (iPPI) through a structure-based virtual ligand screening approach. Our hypothesis is that specific inhibition of the substrate-binding exosites in circulating APC, will result in the relative enhancement of circulating levels of cofactor proteins FV and FVIII without affecting the cytoprotective properties of APC. We hypothesize that these iPPI's could, after having been optimized, (partly) relieve the burden of the existing deficiency and/or reduce the intensity of protein-replacement therapy needed to maintain a haemostatic balance.

\section{Materials and Methods}

\section{Proteins}

Human FV from plasma of a normal individual was prepared as described (7). FXa, a-thrombin, and prothrombin were purchased from Kordia Lab Supplies, The Netherlands. All coagulation factors were of human origin, unless otherwise stated. The anti-human protein C antibodies 0370 and P0374 were from Dako (Denmark) and the APC inhibitory polyclonal antibody used was from Nordic Immunology (Tilburg, The Netherlands). Phospholipid vesicles were prepared as described (22). Synthetic lipids, 1,2 dioleoyl-sn-glycero-3-phosphoserine (DOPS), 1,2 dioleoyl-sn-glycero-3phosphocholine (DOPC) and 1,2 dioleoyl-sn-glycero-3-phosphoethanolamine (DOPE) were obtained from Avanti Polar Lipids (Alabaster, Alabama, USA). Phospholipid 
vesicles were prepared as described previously (23). The chromogenic substrates H-DPhe-Pip-Arg-p-nitroanilide (S-2238) and pyroGlu-Pro-Arg- p-nitroanilide (S2366) were obtained from Chromogenix (Milan, Italy). Ovalbumin was purchased from Sigma (St Louis, USA). Small chemical compounds were purchased from Chembridge (La Jolla, USA). DMSO ( $99.9 \%$ pure) was from Sigma, USA. Corn trypsin inhibitor (CTI) was from Hematologic Technologies, Essex Junction VT, USA and tissue factor (TF) was from Dade-Behring.

\section{Expression and Purification of Recombinant Human Protein $C$}

Two recombinant human protein $\mathrm{C}$ mutants, Y302A/F316A (YFAA variant) and K191A/K192A/K193A/R229A/R230A (5A variant) were produced via PCR-based site-directed mutagenesis of the eukaryotic expression vector pRC/RSV (Invitrogen), expressed in HEK293 cells (CRL-1573ATCC), purified and characterized essentially as described earlier $(24,25)$. In order to produce the $5 \mathrm{~A}$ variant, we first prepared a K191A/ K192A/K193A (3A variant) and a R229A/R230A (2A variant) and recombined the mutated DNA fragments into 1 expression vector containing all five desired missense mutations. We intended to similarly express a fourth mutant, L194A/L228A, however we did not succeed in the expression of this variant since it could be shown that it was not secreted from the HEK293 cells and was degraded intracellularly (not shown). The purity and integrity of the protein C preps were evaluated by SDS-PAGE. Protein C concentrations were quantified by measurement of absorbance at $280 \mathrm{~nm}$ using an absorption coefficient of $14.5(280 \mathrm{~nm}, 1 \%, 1 \mathrm{~cm})$. Protein C was further quantified using a protein C ELISA, employing the anti-human protein C polyclonal antibody Dako 0370 (Dako, Denmark) as a catcher antibody and the horseradish peroxidaseconjugated anti-human-protein C polyclonal DAKO P0374, as a detecting antibody. Dilutions of normal pooled plasma were used to calibrate the assay, assuming that plasma contains $64.5 \mathrm{nM}$ protein C (26).

\section{Activation of Protein C and Catalytic Activity against Small Substrates}

Protein $\mathrm{C}$ variants were activated with bovine thrombin, and the thrombin was removed by ion exchange chromatography, as previously described (27). Complete removal of thrombin was ascertained by the measured absence of a difference in amidolytic activity between measurement with S2238 of the APC preparation, in the presence and absence of hirudin. APC concentrations were determined from the absorbance at $280 \mathrm{~nm}$ and by the above-described protein C ELISA. 


\section{Druggable Pocket Definition}

The detection and the druggabilityofthe binding pockets at the surface of human APC were assessed using the web server DoGSiteScorer (28),FTSite (29) and MetaPocket (30). The APC structure used for the assessment has as PDB code 1AUT.pdb.

\section{Virtual Screening}

The 50,000 compounds of the ChemBridge ExpressPick Diversity set (Oct2007) were prepared as 3D single conformations using Frog (31) and then docked in the contiguous pockets present on the P'side of the catalytic site of APC, toward the area involved in FVa/FVIIIa contact sites using Surflex 2.1 (32). The logprocess command of Surflex 2.1 was then used to rescore the docked poses to weigh the polar and crash contributions in the final docking scores using respectively a threshold of 1 for polar interaction and -2 for crash tolerances. A ranking was made based on this score, and the top 2000 compounds were then manually inspected. Among these 2000 compounds a list of 624 compounds was selected for experimental testing.

\section{Inbibition of APC activity in Plasma}

To analyze that in its plasma environment APC inhibition contributes to overall FVa stability in plasma, and that this inhibition can enhance the lifetime of FVa and hence of thrombin formation, we performed an in vitro thrombin generation experiment in which we measured generation of $\mathrm{FVa}$ and of thrombin in plasma simultaneously. To this end, normal pooled plasma was triggered with $12 \mathrm{pM}$ of relipidated tissue factor (TF) and thrombin generation and FVa generation were followed in time by sampling from the plasma mixture into $20 \mathrm{mM}$ Tris pH7.9, $150 \mathrm{mM} \mathrm{NaCl}, 20 \mathrm{mM}$ EDTA (stopbuffer). Diluted samples in stopbuffer were next assayed for the presence of thrombin using the chromogenic substrate S2238 (Chromogenix) as described earlier (23). Simultaneously, samples were assayed for the presence of FVa activity as described before (7). Triggering of coagulation in plasma was performed in the presence or absence of an APC inhibitory polyclonal antibody (goat-anti-human-APC, GAHu/pC, Nordic Immunology, Tilburg, The Netherlands).

\section{Functional Screening of Small Molecules}

Activity of compounds was initially screened in mixes of 4 different compounds at a concentration of $125 \mu \mathrm{M}$ each (final concentration). Upon positive test outcome the mixture was deconvoluted and the individual compounds were tested. The assay was carried out generally as described before (33): FV from plasma was diluted 1800fold in $25 \mathrm{mM}$ Hepes $\mathrm{pH} 7.5,150 \mathrm{mM} \mathrm{NaCl}$ and $5 \mathrm{mg} / \mathrm{ml} \mathrm{BSA}$ (Buffer I) and was 
activated with thrombin for 15 minutes at $37{ }^{\circ} \mathrm{C}$ in the presence of 10/90 DOPS/ DOPC phospholipid vesicles and calciumchloride. Next, DMSO only or hAPC was added after this had been preincubated with compounds in DMSO diluted in Buffer $\mathrm{I}$, and the inactivation reaction was allowed to proceed for 10 minutes at $37^{\circ} \mathrm{C}$, with the final concentration DMSO during the inactivation being 5,5\% for all test samples. Finally, FVa inactivation was stopped by the addition of prothrombin and FXa, to initiate prothrombin conversion by the prothrombinase complex for 150 seconds, after which the reaction was stopped by the addition of EDTA. Final concentrations of $\mathrm{FXa}$ and prothrombin in the prothrombin activation mixture were $0,3 \mathrm{nM}$ and $0,5 \mu \mathrm{M}$ respectively. The amount of thrombin formed in the presence or absence of APC was quantitated with the chromogenic substrate S-2238 (Chromogenix, Milan, Italy) and from this the percentage of remaining $\mathrm{FVa}$ was calculated and normalized against a similar percentage measured in the absence of compound. The APC concentration (0.6 $\mathrm{nM}$ ) was chosen such that after the $10 \mathrm{~min}$. inactivation, approximately $15 \%$ remaining FVa activity was present in the sample to which APC was added. In the initial screen, $30 \%$ remaining FVa activity was arbitrarily regarded as a positive test outcome and this was encountered in 72 compound mixtures of the 156 tested $(46 \%)$. Next the deconvoluted 288 single compounds were tested individually and normalized ratios for remaining FVa were calculated. During every series of measurements, several blank values (no compound present) as well as positive controls (assay performed in the presence of the inhibitory goat-anti-protein $\mathrm{C}$ antibody) were included to ensure assay performance. All determinations were performed in triplicate.

\section{Factor Va Assay}

FVa activity was determined from the rate of FXa-catalyzed prothrombin activation, as described previously (34). Briefly, prothrombin activation in a reaction mixture containing $0.5 \mu \mathrm{M}$ prothrombin, a limiting concentration of $\mathrm{FVa}(\leq 20 \mathrm{pM}), 5 \mathrm{nM} F X a$, $40 \mu \mathrm{M}$ phospholipid vesicles (10:90, DOPS:DOPC, mol:mol), $0.5 \mathrm{mg} / \mathrm{ml}$ ovalbumin and $3 \mathrm{mM} \mathrm{CaCl}_{2}$, was incubated for $2 \mathrm{~min}$ at $37^{\circ} \mathrm{C}$, after which thrombin was measured from the rate of S-2238 hydrolysis. We verified that the FVa activity assay was not influenced by percentages of DMSO of up to $5,5 \%$.

\section{APC-catalyzed Inactivation of FVa}

FVa inactivation by APC was determined by following the loss of FXa-cofactor activity of FVa in the prothrombinase complex as a function of time as described earlier (34). Since it has been described (35-37) that the targeted exosite in APC is particularly important for cleavage at the predominant Arg506 cleavage site in FVa, analysis of full time courses of inactivation reveal possible cleavage-specific effects of compounds that are more difficult to extract from single time point measurements as our screening 
assay. The full time course analysis was performed using both plasma derived FVa as described for the screening assay above and two different recombinant variants of FVa: FV R506Q/R679Q (abbreviated as RQQ, in which the Arg506 and Arg679 APC cleavage sites were mutated) or FV R306Q/R679Q (abbreviated as QRQ in which Arg306 and Arg679 were mutated to Gln) were prepared and analysed as described (34). These model molecules can only be cleaved by APC at a single cleavage site, at Arg306 and Arg 506 respectively. In short, 20 pM of the activated FV variants was incubated with $25 \mu \mathrm{M}$ phospholipid vesicles (10\% DOPS $/ 90 \% \mathrm{DOPC}$; mol $/ \mathrm{mol}$ ) at 37 ${ }^{\circ} \mathrm{C}$ in $25 \mathrm{mM}$ Hepes (pH7.5),150mM NaCl and $3 \mathrm{mM} \mathrm{CaCl}_{2}$. Next, three aliquots of 90 $\mu \mathrm{l}$ were taken from the reaction mixture to determine the FVa activity in the absence of APC in triplicate. Then, APC was added to the reaction mixture and the mixture was subsequently separated into several $90 \mu \mathrm{l}$ aliquots, one for each time point measured. For the QRQ and RQQ variants, final APC concentrations of $0.1 \mathrm{nM}$ and $5.0 \mathrm{nM}$ were used, respectively. At different time points after the addition of APC, the residual FVa activity was measured in a prothrombinase assay, in which limiting concentrations of FVa $(\leq 20 \mathrm{pM})$, were analyzed. Prewarmed FXa $(0.3 \mathrm{nM}$ in the prothrombinase mixture) and prothrombin $(0.5 \mu \mathrm{M})$ were added to the FVa aliquots and thrombin generation was allowed to continue during 2,5 min after which the reaction was stopped by $>40$ fold dilution in ice-cold EDTA buffer ( $50 \mathrm{mM}$ Tris, $175 \mathrm{mM} \mathrm{NaCl}, 40 \mathrm{mM}$ EDTA, 0.5 $\mathrm{mg} / \mathrm{ml}$ ovalbumin, $\mathrm{pH}$ 7.9). Thrombin was quantified using the chromogenic substrate S2238 (23). Data analysis was performed as detailed earlier (7). The concentration of DMSO was $0,9 \%$ in the prothrombinase mixture for all samples tested.

\section{Surface Plasmon Resonance}

Direct binding analysis was performed on a Biacore T200 surface plasmon resonance apparatus (GE healthcare, USA). To this end we used two different chips, CM5 and CM7 (GE Healthcare, USA), to which we immobilized the different APC or protein C variants by amine coupling as instructed by the manufacturer. The CM5 chip was coated with wild type APC, APC-5A and APC-YFAA such that final coverage of the chip was $\sim 4800$ resonance units per flow cell. Flow cell 1 was not coated and was used as a reference cell. Via a similar method a CM7 chip was coated with APC and PC, at final immobilization levels of 18,000 resonance units. For binding analysis with the CM5 chip, the Biacore was run at $100 \mu \mathrm{l} / \mathrm{min}$, with $60 \mathrm{~s}$ contact time and $140 \mathrm{~s}$ dissociation time in $0.02 \mathrm{M}$ Tris, $\mathrm{pH} 7.4,0.15 \mathrm{M} \mathrm{NaCl}, 2 \mathrm{mM} \mathrm{CaCl}, 1 \% \mathrm{v} / \mathrm{v}$ DMSO, $0.005 \% \mathrm{v} / \mathrm{v}$ Tween 20 (Biacore running buffer). Varying concentrations of compound, as indicated in the results section, were injected. For regeneration we used $0.02 \mathrm{M}$ Tris $\mathrm{pH} 7.4,0.15 \mathrm{M}$ $\mathrm{NaCl}, 2 \mathrm{mM} \mathrm{CaCl}_{2}, 20 \% \mathrm{v} / \mathrm{v}$ DMSO, $0.1 \% \mathrm{v} / \mathrm{v}$ Tween 20 (Biacore regeneration buffer) and raw data were processed by application of a solvent correction as instructed by the Biacore company for DMSO containing samples. Prior to every run, the apparatus was primed 5 times. Binding analyses with the CM7 chip were performed identical to those 
with the CM5 chip, with the exception of the flow rate, which was set to $50 \mu \mathrm{l} / \mathrm{min}$ and the regeneration buffer used was $0.02 \mathrm{M}$ Tris $\mathrm{pH} 7.4,0.15 \mathrm{M} \mathrm{NaCl}, 2 \mathrm{mM} \mathrm{CaCl}, 20 \%$ v/v DMSO, 0.005\% v/v Tween 20. Sensorgrams were analysed with the Bia-evaluation software (version 1.1.1, Biacore company AB, Sweden) using a 1:1 binding model for the interaction between small compounds and APC or PC. Kinetic parameters were obtained from SPR experiments performed with a compound concentration varying from $3.125 \mu \mathrm{M}$ to $200 \mu \mathrm{M}$ and each series was repeated at least two times. Sensorgrams for which the Bia-evaluation software indicated that the kinetic constants could not be uniquely determined were not included in the calculation of $\mathrm{Kd}$ values.

\section{Results}

\section{Druggability Assessment of the Exosite on APC}

The webservers DoGSiteScorer, FTSite and MetaPocket were used to detect and investigate the binding pockets on the surface of APC using the energy minimized $\mathrm{X}$-ray structure of APC (PDB file: 1AUT.pdb after removal of all non-protein atoms). The procedure has highlighted some critical druggable pockets as shown in Figure 1. The first relevant pocket area concerns regions that include the S1 pocket close to the catalytic triad (H211, D257, and S360), and the second major area involves binding cavities on the S'side of the catalytic site including the S4' area and an exosite known to be important for FVa binding (34-40). These complementary subpockets are contiguous and together possess druggable properties with a cumulated volume of around $500 \AA^{3}$ and a surface area of around $900 \AA^{2}$.

\section{Docking Procedure into the APC Exosite and S4' Pocket Area.}

A docking procedure was designed to specifically identify inhibitors of the APC/FVa interaction, applying structure-based virtual screening methodology onto the exosite region mentioned above. This has permitted the in silico selection of a list of 624 compounds. The selection rationale comprised the identification of compounds that should be able to bind to the S4' pocket and/or the exosite as discussed above. The 624 selected compounds were next purchased and subjected to in vitro testing. 

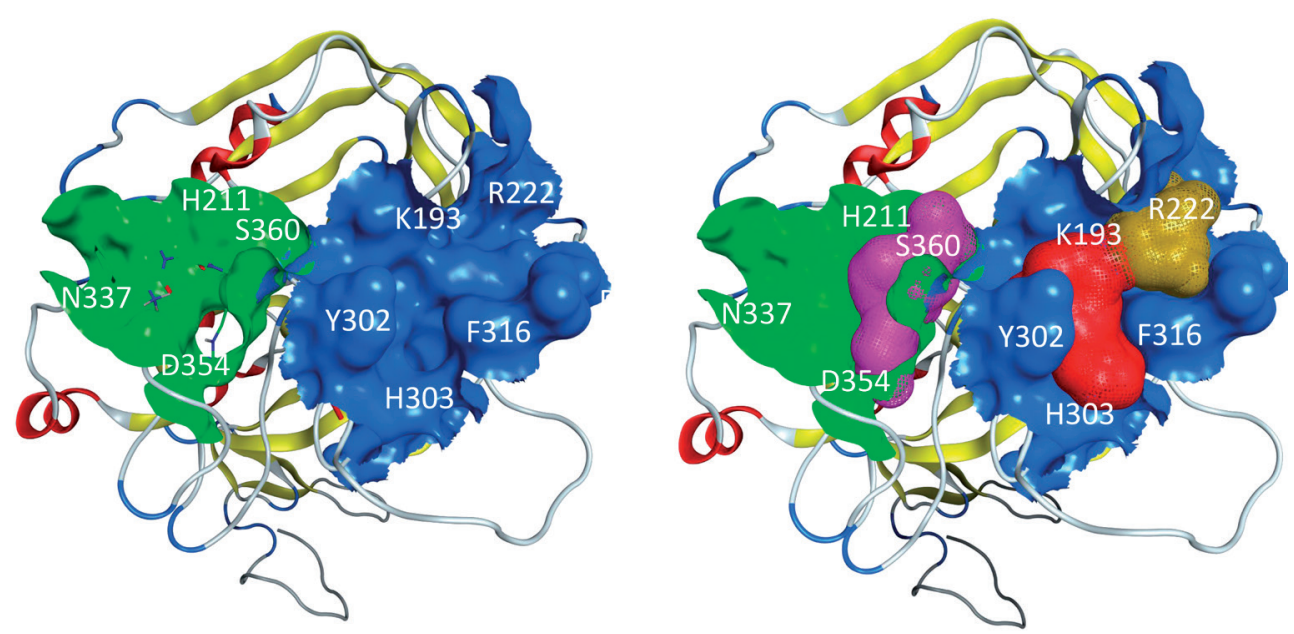

Figure 1. Structure of human APC co-crystallized with a peptide-like inhibitor in the catalytic site and analysis of predicted druggable pockets

Left: The structure of APC is represented as ribbons and molecular surfaces with a view down the active site. The green patch represents the catalytic site occupied by a co-crystallized bound peptide-like inhibitor plunging deep into the S1 pocket with an arginine residue contacting Asp354 at the bottom of the S1 pocket. The blue patch represents the S4' pocket and the exosite identified by binding pocket prediction analysis. Right: predicted druggable pockets at the surface of APC shown as filled mesh in magenta, red and brown. Two major druggable pocket areas were identified with DoGSiteScorer, FTSite and MetaPocket. One region is the well-known catalytic site (shown as a magenta mesh surface) and the other seems to overlap with a known APC binding exosite for factor $\mathrm{V}$ (shown here as a red and brown mesh surface in the area of Y302 and R222). This overall exosite appears to have the required features to bindmacromolecules and low molecular weight molecules. Numbering is according to protein $\mathrm{C}$ nomenclature as presented in (6).

\section{Inbibition of APC Activity in Plasma}

As a model system for in vivo thrombin formation we performed a thrombin generation experiment in a human normal pooled plasma sample in which we measured simultaneously the generation of thrombin and FVa (Figure 2). In the absence of the inhibitory anti-APC antibody, thrombin formation presents with the commonly seen pattern that is characterized by a rapid rise in thrombin formation, which is followed by an equally rapid decline in thrombin activity. These phases coincide with the formation and activity of the prothrombinase complex, which is dependent on the availability of sufficient activated FV (FVa) which is followed by the second phase mainly caused by the inactivation of free thrombin by the circulating plasma inhibitors antithrombin and $\alpha$-2-macroglobulin (41). Since in the initiation phase of thrombin formation, the reaction is dependent upon the availability of FVa, the corresponding FVa generation curve follows that of the formation of thrombin. After the peak in thrombin formation however, although sufficient FVa is available, no further thrombin is formed. 
Importantly, FVa activity in plasma declines over time, a process which is at least partly dependent upon the presence of APC since in the presence of the APC antibody, FVa remains stable for the duration of the experiment. Consequently, in the presence of the antibody, a modest rise in overall thrombin formation is observed (Figure 2), the limited increase in thrombin generation is presumably caused by consumption of prothrombin since we observed that addition of prothrombin, after its consumption from the reaction sample, results in renewed formation of thrombin (data not shown).

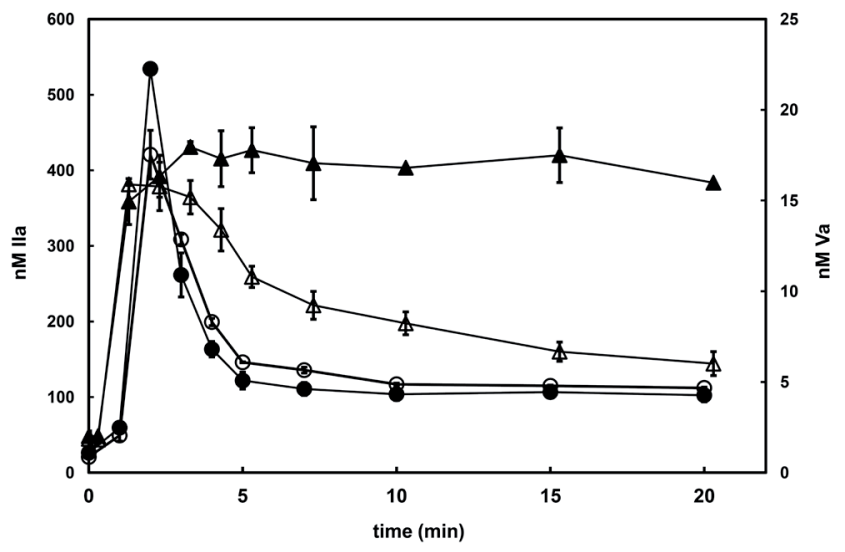

Figure 2. The effect of APC inhibition on thrombin formation and FVa stability in plasma

Normal pooled plasma was extrinsically triggered using $12 \mathrm{pM}$ of relipidated TF and thrombin generation (circles) was followed by sampling at individual time points into an assay specific for thrombin. Simultaneously, the presence of FVawasmonitored (triangles) by sampling into a FVa specific assay (see ref 5). In addition, to the blood plasma was added compensation buffer (open symbols) or an equal volume containing an APC inhibitory antibody (closed symbols).

\section{Expression and Purification of Recombinant Protein C Variants}

Protein $C$ variants were expressed under serum-free conditions at levels of $\sim 5 \mathrm{mg} / \mathrm{l}$ and purified with overall recoveries of $\sim 40 \%$. On SDS-PAGE, all protein $\mathrm{C}$ variants, including the wild type protein $\mathrm{C}$, migrated with a molecular weight of approximately $60 \mathrm{kDa}$. After reduction by addition of $\beta$-mercapto ethanol, the light chain as well as the $\beta$, and $\gamma$ isoforms of the heavy chain were seen (data not shown). Upon activation by thrombin, the heavy-chain isoforms for each of the variants tested shifted to slightly lower migration positions, consistent with full activation (>95\%) as judged by SDS-PAGE. To assess the correct function of the proteins, we further determined activity:antigen ratios of the different APC preps. For wildtype APC, 5A-APC and YFAA-APC these were very comparable, being 1.0; 0.7 and 1.6 respectively. 


\section{Compound Screening}

During the initial screen, mixtures of 4 compounds (at $125 \mu \mathrm{M}$ ) were selected for their ability to inhibit the inactivation of FVa by APC, as determined from the residual FVa activities present after a fixed time of inactivation by APC. Reaction conditions were set such that in the absence of compound, approximately 15\% remaining FVa activity was present in the test sample. Next, for reactions in the presence of compounds, $30 \%$ remaining FVa activity was arbitrarily regarded as a positive test outcome and this was encountered in 72 compound mixtures of the 156 tested $(46 \%)$. Next, positive mixtures were deconvoluted and the 288 resulting single compounds were tested individually in triplicate with the same assay as described for the mixes. From this we selected the top 60 compounds for further screening and these compounds were tested for their ability to inhibit FVa inactivation, as observed from a time course of FVa inactivation (see Figure 3 for a representative example of time courses of $\mathrm{FVa}$ inactivation in the presence of compounds with inhibitory activity towards APC). The analyses of time courses were used to further narrow our selection and the best 20 compounds proceeded to further direct binding analysis by surface plasmon resonance (SPR).

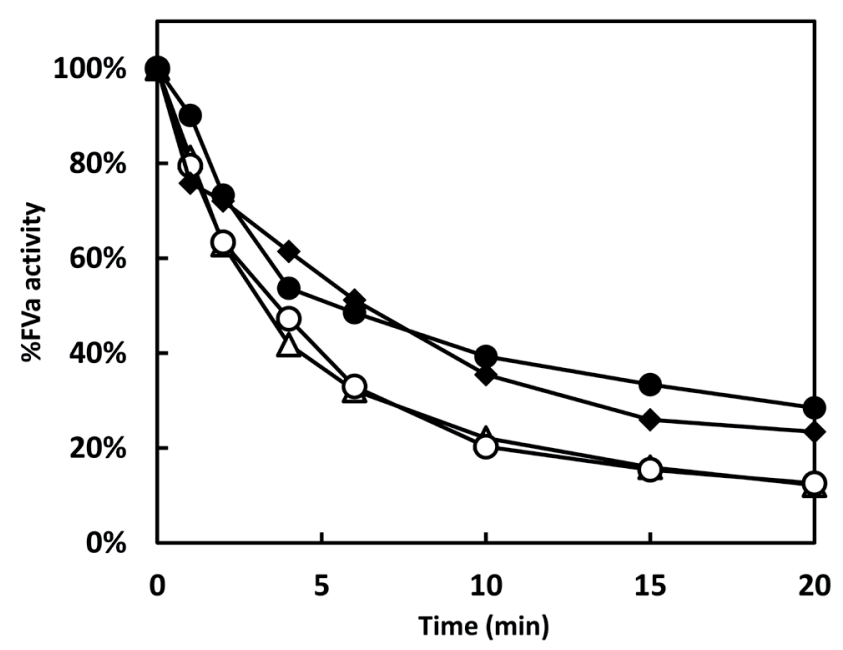

Figure 3. Time course of FVa inactivation, as determined for the 60 best compounds from initial screening

Shown are representative curves obtained for the time courses of inactivation obtained in the absence $(\mathrm{O}, \Delta)$, or presence of $100 \mu \mathrm{M}$ compound G040021 $(\bullet)$ or A070015 ( ). FVa activitywas determinedwith the $\mathrm{FVa}$ assay as described in the methods section, with final concentrations used being: $2.5 \mathrm{nM}$ thrombin, $20 \mathrm{pM}$ FVa, $25 \mu \mathrm{M}$ PL (10/90 DOPS/DOPC), $3 \mathrm{mM}$ calcium ions, $30 \mathrm{pM}$ APC, $0.3 \mathrm{nM}$ FXa, $0.5 \mu \mathrm{M}$ PT. 
Furthermore, we analyzed the effects of compounds on the inactivation of FVa by APC and performed a series of titrations for the top 3 compounds after the screening assay for individual compounds to ascertain a dose-response relationship between the amount of compound present and the extent to which APC is functionally inhibited, thus verifying the methods that were chosen for hit selection. Figure 4 illustrates the outcome of this analysis, which establishes that upon increase of the concentration of compound present, APC is enhancingly inhibited. We observed that the FVa assay used was inhibited to a variable extent at the highest concentrations of compound tested (200 $\mu \mathrm{M}$ and $500 \mu \mathrm{M})$. At $200 \mu \mathrm{M}$ inhibitions of the assay were $34 \%, 7 \%$ and $8 \%$ and at 500 $\mu \mathrm{M}$ inhibition was 85\%,14 and 63\% for A070015, G040021 and G060016 respectively, while the DMSO concentration was constant over the complete range of compound concentrations tested. For all hit molecules identified, we found that the compounds did not change the amidolytic activity of APC towards the small chromogenic substrate S-2366, which implies that binding of the small molecule did not alter the activity of the catalytic site per se (as measured at $200 \mu \mathrm{M} \mathrm{S}-2366$ and $5 \mathrm{nM}$ APC in $50 \mathrm{mM}$ Tris $\mathrm{pH}$ 7.9, $150 \mathrm{mM} \mathrm{NaCl}, 20 \mathrm{mM}$ EDTA, data not shown).

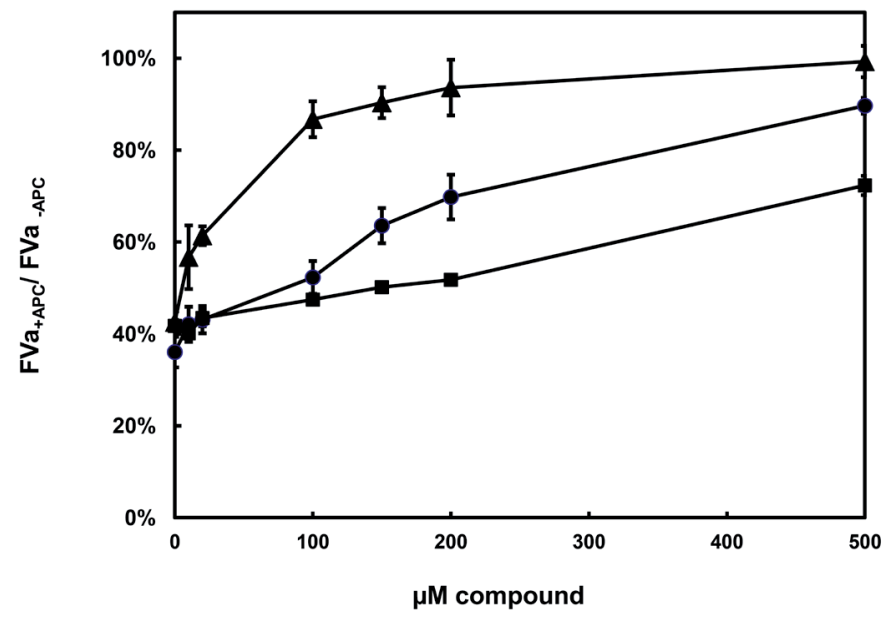

Figure 4. Dose-response curves of top 3 compounds after initial screening of single compounds Increasing amounts of compound are added to the FVa inactivation system as described under Materials and Methods. The ratio of FVa activity present after inactivation by APC and that measured in the absence of APC, while both having the same concentration of compound present, is indicated as a function of the concentration of compound present, with the $\% \mathrm{DMSO}$ being constant over the complete range of compound concentrations tested. Indicated are means \pm SD for three different measurements. Compounds: A070015 (•), G040021 (ム) and G060016 


\section{Direct binding Analysis by SPR}

The 20 best compounds selected after measurement of time courses of FVa inactivation were tested for their ability to bind to APC by surface plasmon resonance with a Biacore T200 apparatus using a CM7 chip. The analysis for the best binding compound, C060018, is shown in Figure 5A and data calculated from similar plots like given in Figure 5 are represented in Table 1. Given that the targeted exosites do not overlap with the activation peptide region in protein $\mathrm{C}$, we also tested the binding of compounds to immobilized PC (Figure 5B). Data shown are the result of a hyperbolic fit to the final levels of binding reached at the end of the binding phase for compound concentrations between 3.125 and $200 \mu \mathrm{M}$.

To verify that compounds were directed at the targeted exosites on the surface of the SP domain of APC, we performed a SPR analysis in which the differential binding of varying concentrations of compounds (between 1 and $100 \mu \mathrm{M}$ ) was studied to wild type APC, 5A-APC and YFAA-APC simultaneously (Figure 6). To allow comparison between compounds, the Kd calculated for W'T-APC binding (see Table 1) was set equal to 1 and the $\mathrm{Kd}$ values calculated for the other APC-variants were expressed relative to that of WT-APC. For a number of compounds (viz. G040021, G050016, G040020, G030014 and B110016) we observed that upon mutation of the targeted binding site, the affinity as determined by SPR lowered by up to $\sim 100-500$ fold. Likewise, over 10fold increases in affinity were also observed (compounds A070015, C060018, E050019 and D050021). While we interpret the change in measured $\mathrm{Kd}$ to reflect an alteration in binding between the immobilised protein and the small compound, it strongly supports the notion that the residues mutated in these particular APC variants are involved in the binding of the small molecules. For three conditions we were unable to uniquely determine quantitative kinetic constants: G040018 binding to 5A-APC and for C020018 binding to $5 \mathrm{~A}-\mathrm{APC}$ and to YFAA-APC.

\section{Cleavage Site Specificity of Compounds}

The different cleavages in FVa have been shown to be mediated via different contacts between the overall large contact area between FVa and APC, in particular three positively charged surface loops of APC, that surround the targeted area are involved in the interaction with the R506 cleavage site in FVa. To further analyse the possible specific effect of our best inhibitory compounds, we used recombinant FVa molecules that can only be cleaved at a single cleavage site (Table 2) to study potential preferred effects by APC-catalyzed cleavage at either Arg506 or Arg306 cleavage in FVa. Table 2 presents the difference between the individual rates of cleavage by APC of mutants RQQ and QRQ, as measured in the same experimental setup as described in the Methods section, in the presence and absence of $100 \mu \mathrm{M}$ compound. It can be observed that the majority of compounds (12 out of 20) influence both the Arg306 and 
a
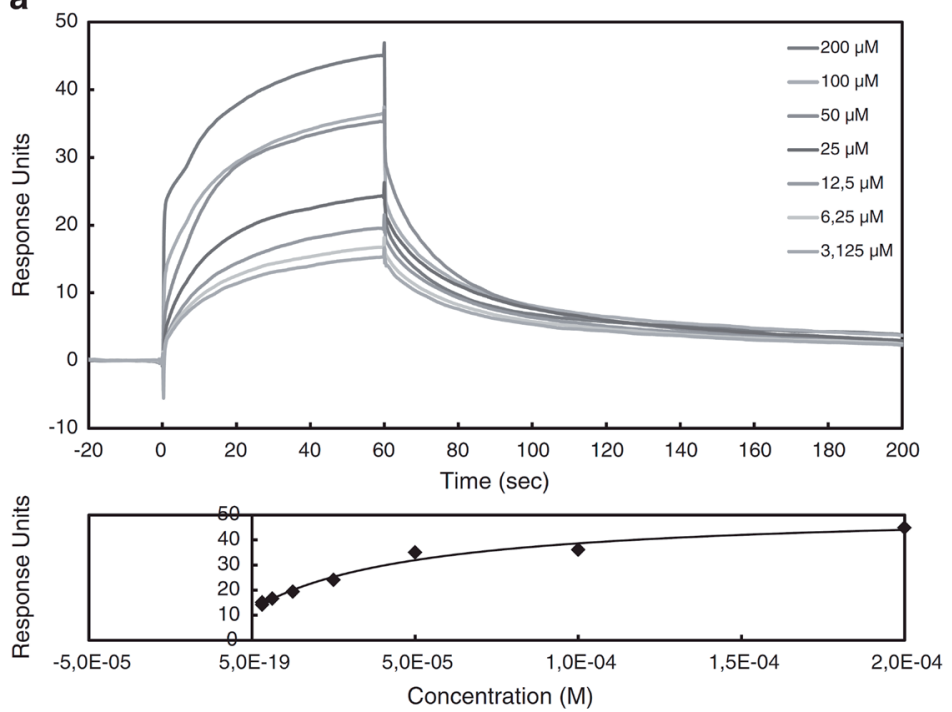

b
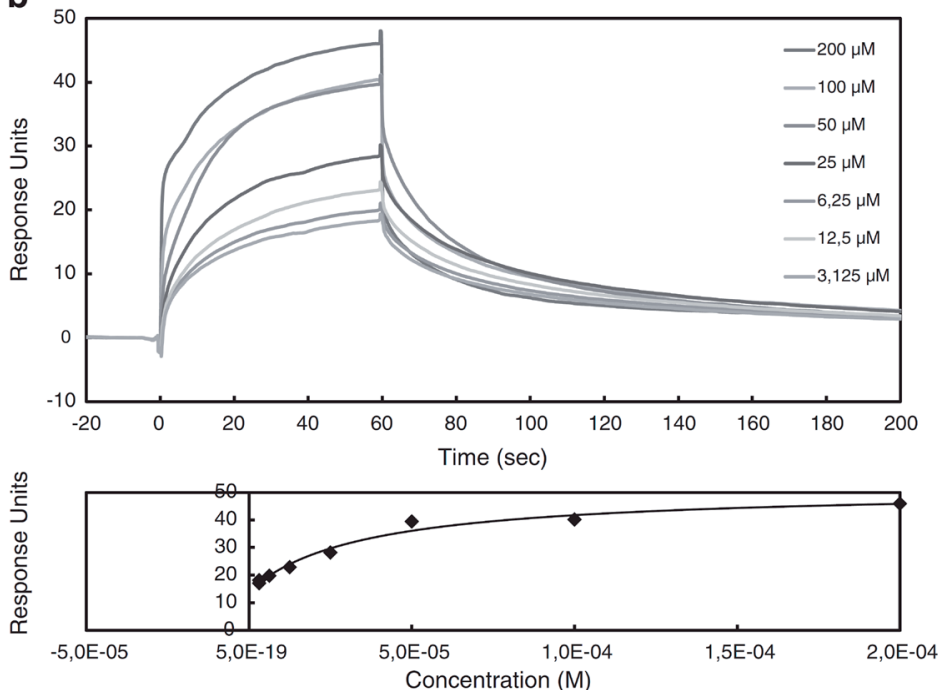

Figure 5.

A) SPR analysis of compound binding to wildtype human APC. (Top) Sensorgrams representing the concentration-dependent binding of compound C060018 to wild type human APC immobilized on a CM7 sensor chip. The association time was 60 seconds and the dissociation time was 140 seconds. Sensorgrams were corrected for binding to the reference cell and for buffer effects. (Bottom) Non-linear affinity analysis of the binding of the compound with human wild type APC. The compound concentration range was 3.125-200 $\mu \mathrm{M}$. B) SPR analysis of compound binding towildtype human PC. (Top) Sensorgrams representing the concentration-dependent binding of compound C060018 towild type human PC immobilized on a CM7 sensor chip. The association time was 60 seconds and the dissociation time was 140 seconds. Sensorgrams were corrected for binding to the reference cell and for buffer effects. (Bottom) Non-linear affinity analysis of the binding of the compound with human wildtype PC. The compound concentration range was $3.125-200 \mu \mathrm{M}$. 


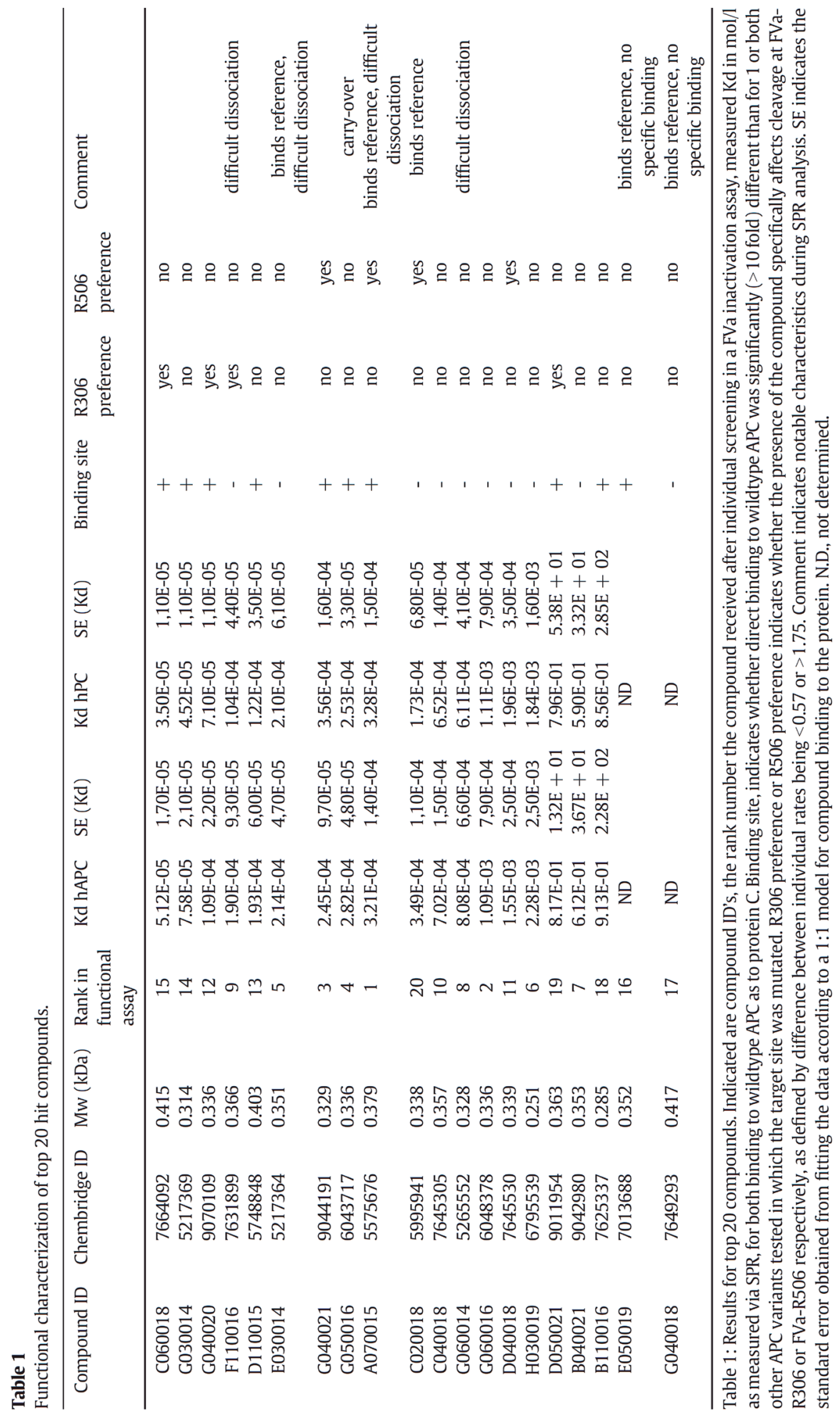


Arg506 cleavage. However, we have detected 4 compounds that preferably influence the cleavage at Arg306 and 4 compounds that appear to preferably influence Arg506 cleavage. Remarkably, the average rank from the activity screening for these compounds was $13,75 \pm 2.14$ and $8,75 \pm 4.33$ respectively (mean \pm SEM), suggesting that the compounds that have a R506 specificity have higher activities than those that preferably inhibit R306 cleavage. Under the conditions chosen here, the most specific compounds presented with a modest 3-5 fold difference in inactivation between the two cleavage sites studied.

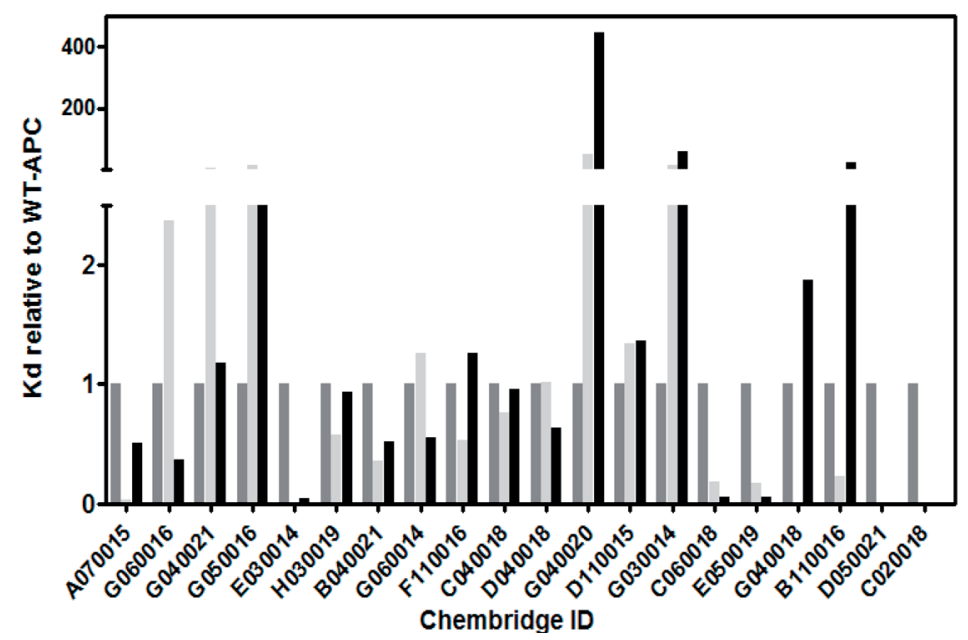

Figure 6. Binding of compounds as quantitated via SPR

The 20 best compounds after first screening were assayed for their affinity to bind to immobilised wild type human APC (WT, dark gray) as well as to 2 APC variants, APC-Y302A/F316A (YFAA, black) and APCK191A/K192A/K193A/R229A/R230A (5A, light gray). For reasons of comparison, Kd values found are expressed relative to those for wild type APC binding. Values indicated are averages of at least 3 analyses. 


\section{Discussion}

In order for APC to proteolyse FVa it needs to form a productive complex with FVa, in the absence of which proteolysis is not possible. In fact, APC has extensive contacts with FVa which by themselves are responsible for the (reversible) loss of FVa cofactor activity prior to cleavage, even in the absence of FVa proteolysis as was observed for an active-site mutated APC (27). The strategy used in the present study is to reduce the inactivation of FVa by prevention of its protein-protein interaction with APC. The goal was to identify small drug-like molecules that bind to APC and inhibit its anticoagulant properties through targeting of the interaction patch on the surface of APC that binds $\mathrm{FVa}$, without targeting the catalytic site. Via this approach one could specifically block the anti-coagulant function of APC but leave intact the cytoprotective properties of APC that depend on the availability of a functional catalytic site. To this end we used various in silico tools (structural bioinformatics and virtual screening) to rationally discover molecules that bind to areas known to be involved in anticoagulant functions. In the first part of the project we therefore scrutinized the regions of APC that should interact with FVa by searching for druggable binding pockets in this area of APC that could overlap with FVa binding sites. To this end, the use of DoGSiteScorer, FTSite and MetaPocket helped us to characterize likely binding zones on the P'side of the catalytic site (see Figure 1). We were able to evaluate the druggability of pockets and subpockets identified in the region of the S4' pocket and of an exosite contiguous to the S4' pocket.

Table 2 Fold-difference between the rates of inactivation at R306 and R506, as influenced by the presence of $100 \mu \mathrm{M}$ compounds.

\begin{tabular}{lllll}
\hline$\#$ & compound ID & 306 cleavage & 506 cleavage & $506 / 306$ ratio \\
\hline 1 & H030019 & 2,01 & 2,12 & 1,05 \\
2 & G040020 & 2,97 & 1,33 & $\mathbf{0 , 4 5}$ \\
3 & D110016 & 2,76 & 2,66 & 0,96 \\
4 & B040021 & 3,01 & 2,87 & 0,95 \\
5 & D110015 & 1,97 & 2,27 & 1,15 \\
6 & G060014 & 2,25 & 1,43 & 0,63 \\
7 & G060016 & 5,04 & 4,88 & 0,97 \\
8 & G050016 & 4,64 & 2,37 & 0,51 \\
9 & G040021 & 1,18 & 2,09 & $\mathbf{1 , 7 7}$ \\
10 & E030014 & 3,22 & 2,05 & 0,64 \\
11 & C040018 & 1,34 & 1,10 & 0,82 \\
12 & F110016 & 5,39 & 2,27 & $\mathbf{0 , 4 2}$ \\
13 & C060018 & 2,03 & 0,96 & $\mathbf{0 , 4 7}$ \\
14 & G030014 & 1,80 & 1,29 & 0,71 \\
15 & A070015 & 1,72 & 3,18 & $\mathbf{1 , 8 5}$ \\
16 & C020018 & 1,01 & 3,00 & $\mathbf{2 , 9 8}$ \\
17 & D040018 & 0,89 & 1,92 & $\mathbf{2 , 1 7}$ \\
18 & D050021 & 1,96 & 0,61 & $\mathbf{0 , 3 1}$ \\
19 & B110016 & 1,66 & 1,19 & 0,71 \\
20 & E050019 & 1,95 & 1,30 & 0,67 \\
\hline
\end{tabular}

The average rates of inactivation at FVa-R306 and FVa-R506, to which these differences are compared were determined as: 4,41 $\pm 0.77 * 10^{6} \mathrm{M}-1 \mathrm{~s}-1$ for FVa-R306 and 5,89 $\pm 1.14 * 10^{7} \mathrm{M}-1 \mathrm{~s}-1$ for FVa-R506 (mean \pm SD, $n=20$ ). Used were the FVa variants RQQ and QRQ. A cleavage value N1 implies that the inactivation is inhibited, which implies that the proteolytic inactivation of FVa is slower than normal. 
These combined contiguous pockets possess druggable properties, meaning that they are accessible and large enough to accommodate the binding of a small molecule, and were therefore the subject of our docking procedure with the goal of identifying low molecular weight and non peptidic modulators of the APC/FVa interaction. Several other studies have highlighted that such targetable exosites are indeed present in blood coagulation serine protease $\mathrm{s}$, as was for instance shown for thrombin $(42,43)$, for FXa (44) or for FVIIa $(45,46)$. However, thus far it was not known if a non-peptidic druglike molecule could bind to the selected APC exosites and influence APC function.

With the intention to specifically identify compounds with predicted binding modes that target the S4' pocket and exosite, we selected 624 compounds after in silico screening through small-molecule docking and tested them for their ability to inhibit the inactivation of FVa by APC. Several molecules demonstrated an effect in the inactivation of FVa. We illustrate the predicted position for two molecules, as they either show a good inactivation profile across different experimental tests and/or because they were sensitive to our site directed mutant APC molecules. Compounds A070015 and G040020 are expected to bind into the S4' pocket and the exosite (see Figure 7). The data presented in Figure 2 illustrate the potential effect of APC inhibition on in vitro thrombin formation and more importantly show that FVa in human plasma is stable, provided that its proteolytic downregulator APC is inhibited. In the absence of an inhibitory antibody against APC, FVa activity readily diminished after full activation of $\mathrm{FV}$, due to the in situ formed APC. In the presence of the inhibitory antibody however, increased formation of thrombin was observed and FVa activity remained stable after plasma activation. These observations open the possibility for potential therapy based on inhibition of APC as the prolonged lifetime of FVa may give rise to increased thrombin generation. These findings are in agreement with earlier attempts to design APC inhibitors that increase the thrombin potential in human plasma $(16,21,47)$ and in particular in plasma from haemophiliacs.

During the initial steps of compound screening we identified a fairly large number of positive mixtures containing anti-APC activity (46\% of the 156 mixtures were regarded as positive). This high percentage has been influenced by several factors: first, we choose a threshold for selection which was twice as high as the assay outcome level measured in the absence of any compound, this is a rather mild criterion which was instigated by our expectation that it would be difficult to detect good protein-protein interaction inhibitors that target the large interaction area between FVa and APC (here computed to be at least $1500 \AA^{2}$ on the serine protease domain of APC). When compared to the surface that can be covered by a small molecule (typically 300-500 $\AA^{2}$ ) the challenge taken up here by us becomes more conceivable. Second, given that molecules may act in synergy, it is possible that the coincidental mixture of more than one weaker inhibitor of APC has caused the overall inhibition to be overestimated. Infact, this has been tested in an experiment where we found that the amount of inhibition by a mixture 


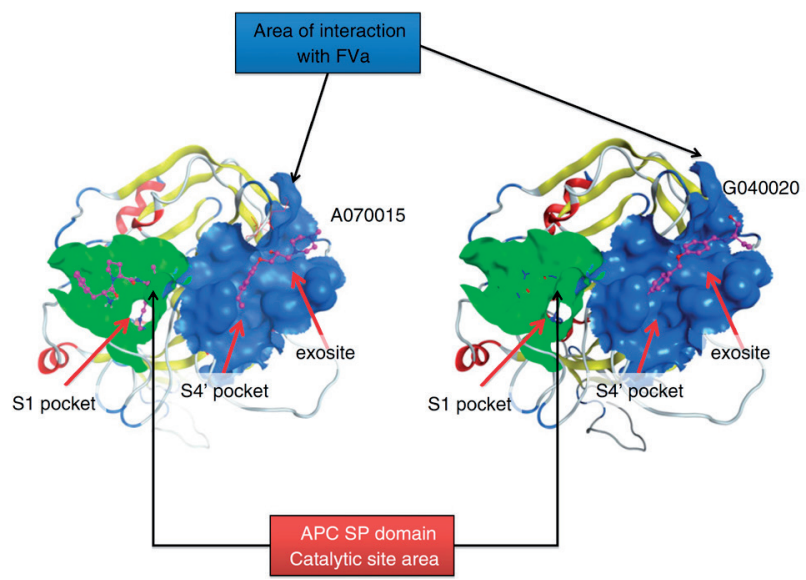

Figure 7. Predicted pose for molecules A070015 and G040020

The overall orientation of APC is as in Figure 1 with a view down the active site. Left, the predicted pose for molecule A070015 is shown, the binding of this molecule is improved in our mutant, suggesting that our pose is most likely acceptable. Indeed, this compound should have a positive charge and the binding could be disfavored by the presence of positive charges in APC, possibly at position R222. Right: The molecule G040020 is shown, several hydrophobic, aromatic and hydrogen bonds are expected to be damagedwhen binding to ourmutant APCmolecules, confirming that thismolecule should fit in this overall area of APC. More detailed explanations about the positioning of the molecules will require NMR and/ or X-ray experiments.

of $50 \mu \mathrm{M}$ each of compounds G060016 and A070015 equaled that by $100 \mu \mathrm{M}$ of either of these compounds (data not shown). Third, the concentration chosen for initial screening $(125 \mu \mathrm{M})$ may be considered as high, which will lead to the detection of moderately active hit molecules.

By means of individual measurements (both functional and direct binding) we selected only the best molecules to be included for further analysis. Moreover, as seen in Figure 4 , we verified that the top 3 compounds after functional screening owed their activity to dose-specific effects rather than to a-specific inhibition of APC, thereby also verifying the validity of our screening assay. While we noticed an influence on the FVa assay, when using high concentrations of compounds, such influence was negligible at the lower concentrations where it was $(14 \%, 4 \%$ and $0 \%$ for A070015, G040021 and G060016 respectively when the assay was performed at $100 \mu \mathrm{M}$ compound). To exclude the possibility of APC inhibition through binding to the active site, we checked that the top 20 compounds did not interfere with proteolysis of the small amidolytic substrate for APC, S2366.

The top 20 molecules after functional screening were analyzed for their ability to bind to APC and its zymogen protein C (see Table 1). The latter is not functional as an anticoagulant but should be accessible to a small compound in the exosites which has been targeted in our virtual screening approach. In agreement with this hypothesis, we 
observed no major differences between the Kd values obtained for binding to APC or to PC. Of interest is the fact that there is little correlation between the observed rank after functional screening and the rank after direct binding analysis for the top 20 molecules (Table1). This may imply that a larger binding surface is available for interaction between the compounds and APC but that a tight binding per se is not sufficient to disturb the interaction between APC and its substrates. This observation is significant since it exemplifies the non-trivial fact that the use of a screening assay that probes the function of a protein by calculation of a ratio of signal measured in the absence- or presence of effector in a multi-component environment, as was the case here, is necessary when the goal of screening experiments is the interference with the function of a protein. In this respect it is worthwhile to mention that use of a functional assay yields not only information on the desired activity of a compound on the intended target protein, but also, the measurements in the absence of APC (and in the presence of compound) in our case yielded information on the potential influence of compounds on the activity of the homologous serine proteases FXa and thrombin, thus hinting at the specificity of the compounds studied. Therefore, we believe that potential binding to off-targets, which are commonly seen in drug discovery campaigns (and which are also seen in many marketed drugs) cannot be excluded but will be noticed and is minimized if a ratio of two functional measurement is determined: one with- and one without the target. In fact several compounds were excluded from further study because they interfered with the assay, in the absence of added APC.

We have sought to gain information on the binding area by use of mutant APC molecules, which contain mutations in the targeted interaction site. Although clearly not for all, we have verified through this approach for 10 out of the top 20 compounds that binding of the compounds is indeed significantly altered when the compound is interacting with the mutant molecule (for 2 out of the top 20 we were not able to determine direct binding parameters due to binding of the molecules to the reference cell). We have observed for 6 compounds a $\sim 100-500$-fold loss in affinity for a given mutated APC, as was most clear for compounds G040020 and G030014 (Figure 7). From the 3D structure of the docked compounds into the wild-type APC structure we can explain this loss of affinity results from reduced or lost hydrophobic/aromatic and hydrogen bond interactions between the compound and the mutated binding site. On the other hand, increase in affinity, as was observed for 4 different compounds could be explained by an enlargement of the binding site and reduced positive charge repulsion through the mutation which facilitates the binding of compounds such as A070015, C060018, E050019 and D050021 (Figure 7). The targeted area has been described to be involved particularly in the cleavage of FVa at position Arg506. Therefore, we measured the individual rates of FVa inactivation at either Arg306 or Arg506, the two predominant cleavage sites in FVa, by use of recombinant FVa molecules that can only be cleaved at a single position. We observed a predominant effect for cleavage at 
Arg506 in FVa for 4/20 compounds tested. Moreover, we observed 4/20 molecules to affect preferably Arg306 as well. From this we conclude that apparently, regions on the surface of APC, that are obscured by the binding of these compounds can likewise be involved in binding at Arg306 as they can be in binding at Arg506. This is in line with earlier observations by us and others who have concluded that a large interaction area is involved in the interaction between APC and FVa and that the epitopes important for cleavage at Arg306 or Arg506 do not fully overlap (27, 34-38, 48). The affinities of the molecules found can be described as of rather modest affinity and are lower than those found for peptide-like compounds (21) or for APC RNA aptamers (49) or ssDNA aptamers (47) who found affinities of $800 \mathrm{nM}, 100 \mathrm{nM}$ and $0.43 \mathrm{nM}$ respectively. Yet, the biological function of a protein-protein interaction can be efficiently modulated even if small molecule binders are not of high affinity and there are many advantages to work with low molecular weight compounds as in general their bioavailability is much higher than for peptides and aptamers.

Taken together we show here, for the first time, that it is possible to discover small proteinprotein interaction inhibitors through a structure-based virtual screening approach of the interaction between coagulation factor Va and APC. The molecules discovered have weak to moderate binding affinities but are able to inhibit the inactivation of the natural substrate FVa, in a system that contains FVa and APC but also albumin. This work could provide a basis on which these molecules may be further developed into orally available small molecules that could be used to increase in vivo thrombin generation by specific inhibition of the anticoagulant properties of APC.

\section{Acknowledgments}

The financial support by an unrestricted Bayer Haemophilia project grant, The Netherlands Organization for Scientific Research (NWO, grants no. 91107006 and 91112016) and the Cardiovascular Research Institute Maastricht (CARIM) are greatly acknowledged. Supports from Inserm and the University Paris Diderot are also appreciated. 


\section{Appendix}

Oligonucleotides used in the preparation of protein $\mathrm{C}$ mutants.

\begin{tabular}{lll}
\hline Mutant & Oligo name & Sequence \\
\hline $2 \mathrm{~A}$ & hPC_RR229/230AA $(+)$ & GAGTATGACCTGGCGGCCTGGGAGAAGTG \\
& hPC_RR229/230AA $(-)$ & CACTTCTCCCAGGCCGCCAGGTCATACTC \\
$3 \mathrm{~A}$ & hPC_KKKK74/75/76AAA $(+)$ & CTGGACTCCGCGGCGGCGCTGGCCTGC \\
& hPC_KKKK74/75/76AAA $(-)$ & GCAGGCCAGCGCCGCCGCGGAGTCCAG \\
YFAA & hPC_F316 $(+)$ & ACCGCACCGCCGTCCTCAACTTCATC \\
& hPC_F316 $(-)$ & GATGAAGTTGAGGACGGCGGTGCGGT \\
& hPC_Y302A $(+)$ & GGGCTGGGGCGCACACAGCAGCCG \\
& hPC_Y302A $(-)$ & CGGCTGCTGTGTGCGCCCCAGCCC \\
\hline
\end{tabular}




\section{References}

1 Wildhagen KC, Lutgens E, Loubele ST, ten Cate H, Nicolaes GA. The structure-function relationship of activated protein C. Lessons from natural and engineered mutations. Thromb Haemost 2011;106:1034-45.

2 Marlar RA, Neumann A. Neonatal purpura fulminans due to homozygous protein $\mathrm{C}$ or protein $\mathrm{S}$ deficiencies. Semin Thromb Hemost 1990;16:299-309.

3 Nicolaes GAF, Dahlback B. Congenital and acquired activated protein C resistance. Semin Vasc Med 2003;3:33-46.

4 Griffin JH, Fernandez JA, Gale AJ, Mosnier LO. Activated protein C. J Thromb Haemost 2007;5(Suppl. 1):73-80.

5 Espana F, Zuazu I,Vicente V,Estelles A, Marco P, Aznar J. Quantification of circulating activated protein $\mathrm{C}$ in human plasma by immunoassays-enzyme levels are proportional to total protein $\mathrm{C}$ levels. Thromb Haemost 1996;75:56-61.

6 Mather T, Oganessyan V, Hof P, Huber R, Foundling S, Esmon C, et al. The 2.8 A crystal structure of Gla-domainless activated protein C. EMBO J 1996;15:6822-31.

7 Nicolaes GAF, Tans G, Thomassen MC, Hemker HC, Pabinger I, Varadi K, et al. Peptide bond cleavages and loss of functional activity during inactivation of factor Va and factor VaR506Q by activated protein C. J Biol Chem 1995;270:21158-66.

8 Lu D, Kalafatis M, Mann KG, Long GL. Comparison of activated protein C/protein S-mediated inactivation of human factor VIII and factor V. Blood 1996;87:4708-17.

9 Mosnier LO, Zlokovic BV, Griffin JH. The cytoprotective protein C pathway. Blood 2007;109:316172.

10 Griffin JH, Zlokovic BV, Mosnier LO. Protein C anticoagulant and cytoprotective pathways. Int J Hematol 2012;95:333-45.

11 Dahlback B, Carlsson M, Svensson PJ. Familial thrombophilia due to a previously unrecognized mechanism characterized by poor anticoagulant response to activated protein $\mathrm{C}$ : prediction of a cofactor to activated protein C. Proc Natl Acad Sci U S A 1993;90:1004-8.

12 Bertina RM, Koeleman BP, Koster T, Rosendaal FR, Dirven RJ, de Ronde H, et al. Mutation in blood coagulation factor $V$ associated with resistance to activated protein C. Nature 1994;369:64-7.

13 Dahlback B, Hildebrand B. Inherited resistance to activated protein C is corrected by anticoagulant cofactor activity found to be a property of factor V. Proc Natl Acad Sci U S A 1994;91:1396-400.

14 Shen L, Dahlback B. Factor V and protein $S$ as synergistic cofactors to activated protein $\mathrm{C}$ in degradation of factor VIIIa. J Biol Chem 1994;269:18735-8.

15 Bos MH, Meijerman DW, van der Zwaan C, Mertens K. Does activated protein C-resistant factor V contribute to thrombin generation in hemophilic plasma? J Thromb Haemost 2005;3:522-30.

16 Butenas S, Orfeo T, Kalafatis M, Mann KG. Peptidomimetic inhibitors for activated protein C: implications for hemophilia management. J Thromb Haemost 2006;4:2411-6.

17 Lee DH, Walker IR, Teitel J, Poon MC, Ritchie B, Akabutu J, et al. Effect of the factor V Leiden mutation on the clinical expression of severe hemophilia A.Thromb Haemost 2000;83:387-91.

18 Nichols WC, Amano K, Cacheris PM, Figueiredo MS, Michaelides K, Schwaab R, et al. Moderation of hemophilia A phenotype by the factor V R506Q mutation. Blood 1996;88:1183-7.

19 SchlachtermanA,Schuettrumpf J,LiuJH, Furlan FreguiaC,TosoR,PonczM, et al. Factor V Leiden improves in vivo hemostasis in murine hemophilia models. J Thromb Haemost 2005;3:2730-7. 
20 van 't Veer C, Golden NJ, Kalafatis M, Simioni P, Bertina RM, Mann KG. An in vitro analysis of the combination of hemophilia A and factor V(LEIDEN). Blood 1997;90:3067-72.

21 De Nanteuil G, Gloanec P, Beguin S, Giesen PL, Hemker HC, Mennecier P, et al. Low molecular weight activated protein $\mathrm{C}$ inhibitors as a potential treatment for hemophilic disorders. J Med Chem 2006;49:5047-50.

22 Nicolaes GAF, Villoutreix BO, Dahlback B. Partial glycosylation of Asn2181 in human factor V as a cause of molecular and functional heterogeneity. Modulation of glycosylation efficiency by mutagenesis of the consensus sequence for N-linked glycosylation. Biochemistry 1999;38:13584-91.

23 Rosing J, Tans G, Govers-Riemslag JW, Zwaal RF, Hemker HC. The role of phospholipids and factor Va in the prothrombinase complex. J Biol Chem 1980;255:274-83.

24 Friedrich U, Potzsch B, Preissner KT, Muller-Berghaus G, Ehrlich H. Calcium-dependent activation of protein $\mathrm{C}$ by thrombin/thrombomudulin: role of negatively charged amino acids within the activation peptide of protein C. Thromb Haemost 1994;72:567-72.

25 Sun YH, Shen L, Dahlback B. Gla domain-mutated human protein C exhibiting enhanced anticoagulant activity and increased phospholipid binding. Blood 2003;101:2277-84.

26 Griffin JH, Evatt B, Zimmerman TS, Kleiss AJ, Wideman C. Deficiency of protein C in congenital thrombotic disease. J Clin Invest 1981;68:1370-3.

27 Nicolaes GAF, Bock PE, Segers K, Wildhagen KC, Dahlback B, Rosing J. Inhibition of thrombin formation by active site mutated (S360A) activated protein C. J Biol Chem 2010;285:22890-900.

28 Volkamer A, Kuhn D, Rippmann F, Rarey M. DoGSiteScorer: a web server for automatic binding site prediction, analysis and druggability assessment. Bioinformatics 2012;28:2074-5.

29 Ngan CH, Hall DR, Zerbe B, Grove LE, Kozakov D, Vajda S. FTSite: high accuracy detection of ligand binding sites on unbound protein structures. Bioinformatics 2012;28:286-7.

30 Huang B. MetaPocket: a meta approach to improve protein ligand binding site prediction. OMICS 2009;13:325-30.

31 Leite TB, Gomes D, Miteva MA, Chomilier J, Villoutreix BO, Tuffery P. Frog: a FRee Online druG 3D conformation generator. Nucleic Acids Res 2007;35:W568-72.

32 Jain AN. Surflex-Dock 2.1: robust performance from ligand energetic modeling, ring flexibility, and knowledge-based search. J Comput Aided Mol Des 2007;21:281-306.

33 Nicolaes GAF, Thomassen MC, van Oerle R, Hamulyak K, Hemker HC, Tans G, et al. A prothrombinase-based assay for detection of resistance to activated protein C. Thromb Haemost 1996;76:404-10.

34 Segers K, Dahlback B, Rosing J, Nicolaes GAF. Identification of surface epitopes of human coagulation factor $\mathrm{Va}$ that are important for interaction with activated protein $\mathrm{C}$ and heparin. J Biol Chem 2008;283:22573-81.

35 Gale AJ, Heeb MJ, Griffin JH. The autolysis loop of activated protein C interacts with factor Va and differentiates between the Arg506 and Arg306 cleavage sites. Blood 2000;96:585-93.

36 Friedrich U, Nicolaes GAF, Villoutreix BO, Dahlback B. Secondary substrate-binding exosite in the serine protease domain of activated protein C important for cleavage at Arg-506 but not at Arg-306 in factor Va. J Biol Chem 2001;276:23105-8.

37 Gale AJ, Tsavaler A, GriffinJH. Molecular characterization of an extended binding site for coagulation factor Va in the positive exosite of activated protein C. J Biol Chem 2002;277:28836-40.

38 Nicolaes GAF, Sorensen KW, Friedrich U, Tans G, Rosing J, Autin L, et al. Altered inactivation pathway of factor Va by activated protein C in the presence of heparin. Eur J Biochem 2004;271:2724-36.

39 Takeyama M,Wintermute JM, ManithodyC, Rezaie AR, Fay PJ. Variable contributions of basic residues forming an APC exosite in the binding and inactivation of factor VIIIa. Biochemistry 2013;52:222835. 
40 Cramer TJ, Gale AJ. Function of the activated protein C (APC) autolysis loop in activated FVIII inactivation. Br J Haematol 2011;153:644-54.

41 Hemker HC, AlDieri R, De Smedt E, Beguin S. Thrombin generation, a function test of the haemostatic-thrombotic system. Thromb Haemost 2006;96:553-61.

42 Padmanabhan K, Padmanabhan KP, Ferrara JD, Sadler JE, Tulinsky A. The structure of alphathrombin inhibited by a 15-mer single-stranded DNA aptamer. J Biol Chem 1993;268:17651-4.

43 Holland CA, Henry AT, Whinna HC, Church FC. Effect of oligodeoxynucleotide thrombin aptamer on thrombin inhibition by heparin cofactor II and antithrombin. FEBS Lett 2000;484:87-91.

44 Murakami MT, Rios-Steiner J, Weaver SE, Tulinsky A, Geiger JH, Arni RK. Intermolecular interactionsand characterization of the novel factor Xa exosite involved inmacromolecular recognition and inhibition: crystal structure of human Gla-domainless factor Xa complexed with the anticoagulant protein NAPc2 from the hematophagous nematode Ancylostoma caninum. J Mol Biol 2007;366:60210.

45 Dennis MS, Eigenbrot C, Skelton NJ, Ultsch MH, Santell L, Dwyer MA, et al. Peptide exosite inhibitors of factor VIIa as anticoagulants. Nature 2000;404:465-70.

46 Carlsson K, Persson E, Carlsson U, Svensson M. Inhibitors of factor VIIa affect the interface between the protease domain and tissue factor. Biochem Biophys Res Commun 2006;349:1111-6.

47 Muller J, Isermann B, Ducker C, Salehi M, Meyer M, Friedrich M,et al. An exosite-specific ssDNA aptamer inhibits the anticoagulant functions of activated protein $\mathrm{C}$ and enhances inhibition by protein C inhibitor. Chem Biol 2009;16:442-51.

48 Yang L, Manithody C, Rezaie AR. Contribution of basic residues of the 70-80-loop to heparin binding and anticoagulant function of activated protein C. Biochemistry 2002;41:6149-57.

49 Gal SW, Amontov S, Urvil PT, Vishnuvardhan D, Nishikawa F, Kumar PK, et al. Selection of a RNA aptamer that binds to human activated protein $\mathrm{C}$ and inhibits its protease function. Eur $\mathrm{J}$ Biochem 1998;252:553-62. 


\section{Chapter 4}

\section{Effects of exogenous recombinant APC in mouse models of ischemia reperfusion injury and of atherosclerosis}

Karin C.A.A. Wildhagen ${ }^{1}$, Roy Schrijver ${ }^{1}$, Linda Beckers ${ }^{2}$, Hugo ten Cate ${ }^{1,3}$, Chris P.M. Reutelingsperger ${ }^{1}$, Esther Lutgens ${ }^{2}$, Gerry A.F. Nicolaes ${ }^{1}$

1. Department of Biochemistry, Cardiovascular Research Institute Maastricht, Maastricht University, The Netherlands

2. Department of Medical Biochemistry, Academic Medical Center, Amsterdam, The Netherlands

3. Department of Internal Medicine, Laboratory for Clinical Thrombosis and Haemostasis, Cardiovascular Research Institute Maastricht, Maastricht University, The Netherlands

PLoS One. 2014;9(7):e101446. 


\section{Abstract}

Activated protein C (APC) is a serine protease that has both anticoagulant and cytoprotective properties. The cytoprotective effects are protease activated receptor 1 (PAR-1) and endothelial protein C receptor (EPCR) dependent and likely underlie protective effects of APC in animal models of sepsis, myocardial infarction and ischemic stroke. S360A-(A)PC, a variant (A)PC that has no catalytic activity, binds EPCR and shifts pro-inflammatory signaling of the thrombin-PAR-1 complex to antiinflammatory signaling.

In this study we investigated effects of human (h)wt-PC, hS360A-PC, hwt-APC and hS360A-APC in acute (mouse model of acute myocardial ischemia/reperfusion (I/R) injury) and chronic inflammation (apoE $\mathrm{E}^{-/-}$mouse model of atherosclerosis).

All $h(A) P C$ variants significantly reduced myocardial infarct area $(\mathrm{p}<0.05)$ following I/R injury. IL-6 levels in heart homogenates did not differ significantly between sham, placebo and treatment groups in I/R injury. None of the h(A)PC variants decreased number and size of atherosclerotic plaques in apo: $\mathrm{E}^{-/-}$mice. Only hS360A-APC slightly affected phenotype of plaques. IL-6 levels in plasma were significantly $(\mathrm{p}<0.001)$ decreased in hwt-APC and hS360A-PC treated mice. In the last group levels of monocyte chemotactic protein 1 (MCP-1) were significantly increased $(\mathrm{p}<0.05)$.

In this study we show that both hwt and hS360A-(A)PC protect against acute myocardial I/R injury, which implies that protection from I/R injury is independent of the proteolytic activity of APC. However, in the chronic atherosclerosis model hwt and hS360-(A)PC had only minor effects. When the dose, species and mode of (A)PC administration will be adjusted, we believe that (A)PC will have potential to influence development of chronic inflammation as occurring during atherosclerosis as well. 


\section{Introduction}

Protein $\mathrm{C}(\mathrm{PC})$ circulates as an inactive zymogen that can be activated by thrombin bound to the endothelium associated transmembrane receptor thrombomodulin. When PC is bound to the endothelial protein $C$ receptor (EPCR), its activation is enhanced by 20 -fold (1). Activated protein C (APC) is a serine protease that, together with its cofactors protein $\mathrm{S}$ and factor $\mathrm{V}(\mathrm{FV})$, regulates thrombin formation by proteolysis of the active coagulation factors $\mathrm{V}$ (FVa) and VIII (FVIIIa) (2-5). The anticoagulant PC pathway is vital to normal haemostasis, as indicated by the increased risk for thromboembolic events in heterogeneous PC deficiency $(6,7)$ and the life threatening thrombotic complications observed, even in the presence of aggressive anticoagulant therapy, in homozygous PC deficiency $(8,9)$.

APC has initially been identified and characterized as an anticoagulant protein; however recent interest has focused more on the cytoprotective functions of APC. APC has anti-inflammatory, anti-apoptotic and endothelial barrier stabilizing effects in different cell types, that are protease activated receptor 1 (PAR-1) and EPCR-dependent $(10,11)$. These cellular effects, rather than the anticoagulant activity of APC, likely explain the protective effects of APC observed in animal studies of sepsis, lung injury, myocardial infarction and ischemic stroke (12-16).

The active site of APC contains the conserved catalytic triad: residues H211, D257 and S360. Mutation of the active site serine to alanine (S360A) yields a protein that is not able to cleave substrates, but still retains some anticoagulant activity through its ability to compete with activated coagulation factors X and IX for binding to FVa and FVIIIa, respectively $(17,18)$. The cell surface receptor PAR-1 is involved in mediation of the cell-protective properties of APC. S360A-APC, however fails to cleave PAR-1 and is therefore regarded as being devoid of cytoprotective effects (19). Thrombin is also able to cleave PAR-1 and in contrast to APC cleaved PAR-1, thrombin cleaved PAR-1 causes permeability-enhancing and pro-inflammatory effects via $G_{q}$ and $G_{12 / 13}$ mediated signaling (20). Interestingly, it has been shown that binding of APC, PC as well as S360A-PC to EPCR, is associated with PAR-1 coupling to a G(i/o) family of G-proteins, resulting in initiation of protective signaling after cleavage of PAR-1 by either APC or thrombin (21). Thus, this implies that protective PAR-1 signaling can also be initiated by PC and by proteolytically inactive S360A-(A)PC. Therefore we hypothesize that not only human wild type (hwt)-APC, but also hwt-PC, hS360APC and hS360A-APC may initiate cytoprotective signaling in vivo via EPCR-dependent modulation of PAR-1 signaling.

In the present study we have investigated the cytoprotective effects of human hwtPC, hS360A-PC, hwt-APC and hS360A-APC in two different cardiovascular relevant mouse models: myocardial ischemia/reperfusion (I/R) injury and atherosclerosis. hAPC is very similar to murine (m) APC (69\% sequence identity) (22). It is known that $\mathrm{mAPC}$ is significantly more potent than hAPC as an anticoagulant in mice (15), but we 
hypothesize that anticoagulation is not necessary for protection in our models. Previous studies have shown that hAPC has positive effects on lesion volume, inflammation, vascular permeability and neurological function in murine models studying $\mathrm{I} / \mathrm{R}$ injury and stroke (23-27).

Inflammation plays a key role in the pathogenesis of both myocardial I/R injury and atherosclerosis models in the current study, although the sequence of immune cell infiltration and the characteristics of the immune cell infiltrate are markedly different. After acute myocardial I/R, neutrophils migrate into the infarct zone within 24 hours, where they release granular enzymes and reactive oxygen species and cause vascular plugging (28). In atherosclerosis, mainly T-cells and monocytes are recruited into the plaque, where monocytes mature into macrophages and finally foam cells. These immune cells release cytokines within the plaque and promote the expression of matrix metalloproteinases that break down collagen and thereby make plaques more vulnerable (29). We hypothesize that hwt-(A)PC and hS360A-(A)PC will protect against acute inflammation in the myocardial I/R injury model and chronic inflammation in the atherosclerosis model.

\section{Materials \& Methods}

\section{Expression and purification of recombinant human (S360A)-PC}

Recombinant hS360A-PC was obtained by PCR-based site-directed mutagenesis of hwt-PC cDNA. The cDNA of hwt-PC and hS360A-PC were inserted into the eukaryotic expression vector pRc/CMV (Invitrogen), transfected into HEK293 cells (CRL-1573 ATCC) and recombinant $\mathrm{h}(\mathrm{S} 360 \mathrm{~A})-\mathrm{PC}$ was purified and characterized as described earlier $(30,31)$. The purity and integrity of $\mathrm{h}(\mathrm{S} 360 \mathrm{~A})-\mathrm{PC}$ was evaluated by SDS-PAGE (Figure 1). h(S360A)-PC concentrations were quantified by measurement of absorbance at $280 \mathrm{~nm}$ using an absorption coefficient of $14.5(280 \mathrm{~nm}, 1 \%, 1 \mathrm{~cm})$ and by ELISA, employing the horse anti-human PC polyclonal antibody PAHPC-H (HTI), as a catcher antibody and the horseradish peroxidase-conjugated anti-human PC polyclonal Dako P0374 as a detecting antibody (18).

\section{Activation of $h(S 360 A)-P C$ and catalytic activity against small substrates}

hwt-PC and hS360A-PC were activated with a $1 \mathrm{ml}$ HiTrap NHS-Activated HP column (GE Healthcare) to which 50 Units of carrier-free Protac (Pentapharm) had prior been conjugated. Up to $0.5 \mathrm{mg}$ of $\mathrm{h}(\mathrm{S} 360 \mathrm{~A})-\mathrm{PC}$ was incubated for 2 hours on the column at room temperature, and was next eluted and concentrated with a $10 \mathrm{~K}$ Macrosep Advance Centrifugal Device (Pall Life Sciences). Complete activation of the hwt-PC was ascertained by measuring the amidolytic activity of APC with S-2366 (Chromogenix) 
directly and after 60 minutes incubation in the presence of additionally added (0.05 units $/ \mathrm{ml}$ final concentration) Protac. We confirmed the absence of Protac from the APC preps generated. For both hwt- and hS360A-PC, completion of activation was verified by SDS PAGE analysis (Figure 1). APC concentrations were determined from the above-described PC ELISA.

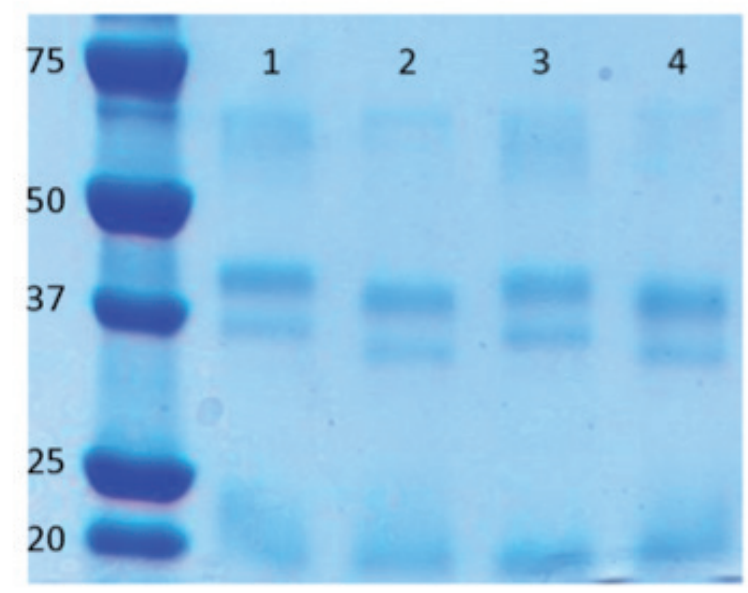

Figure 1. SDS page gel of human wt- and S360A-(A)PC

Reduced samples of human wt-PC (lane 1), wt-APC (lane 2), S360A-PC (lane 3) and S360A-APC (lane 4) were loaded on a $10 \%$ SDS PAGE gel and afterwards stained with Coomassie Brilliant Blue.

Mice

All animal experiments were approved by the Animal Ethics Committee of Maastricht University. All mice were housed and bred according to institutional guidelines and were fed a normal chow diet. For the myocardial I/R experiment male C57BL/6 mice (Charles River Laboratories) of 8 weeks old and $25 \mathrm{~g}$ were used ( $\mathrm{n}=13$ per group). For the atherosclerosis experiments, 21-week old male $\mathrm{ApoE}^{-/}$mice were used (Charles River Laboratories/in house breeding facility Maastricht University). Treatment, twice weekly, with intraperitoneal (i.p.) injections of saline ( $\mathrm{n}=20)$ or $0.2 \mathrm{mg} / \mathrm{kg}$ of hwt-PC ( $\mathrm{n}=13)$, hS360A-PC $(\mathrm{n}=12)$, hwt-APC $(\mathrm{n}=16)$ or hS360A-APC $(\mathrm{n}=16)$ started at week 21 and was continued for 8 weeks. The $0.2 \mathrm{mg} / \mathrm{kg}$ dose has been proven protective in models of myocardial I/R injury (23), hepatic I/R injury (25) and stroke $(15,32,33)$.

\section{Procedure myocardial I/R}

Myocardial I/ $R$ was performed as described earlier (34). In short, mice were anaesthetized with isoflurane and were ventilated during the ischemic period. Ischemia was induced by ligation of the left anterior descending (LAD) artery for 60 minutes followed by 2 hours of reperfusion. hwt-PC, hS360A-PC, hwt-APC or hS360A-APC $(0.4 \mathrm{mg} / \mathrm{kg})$ or 
placebo (saline) were administered intravenously (i.v.) both 15 minutes after induction of ischemia and 5 minutes after induction of reperfusion. After reperfusion, the animals were anaesthetized as described earlier. Heart tissue was collected for determination of infarct size ( $\mathrm{n}=6$ per group) or interleukin (IL)-6 levels ( $\mathrm{n}=7$ per group).

\section{Myocardial I/R: determination of infarct size}

Infarct size was determined by Evans Blue/triphenyl tetrazolium chloride (TTC) staining (35). In brief, after reperfusion, the LAD was re-ligated and Evans Blue dye was injected i.v. and was allowed to circulate for 2 min to identify the area at risk (AAR). After excision of the heart, it was cut into 0.5-mm-thick slices and TTC was added to mark the viable cells within the AAR red. The non-viable cells remained pale and defined the area of infarction (AOI). Pictures of the slices were taken and were analyzed by Adobe Photoshop CS2 to determine infarct sizes (AOI/AAR ratios).

\section{Myocardial I/R: determination of IL-6 levels}

Hearts of mice were excised, lyophilized and the resulting dry heart powder was dissolved in glucopyranoside. After determination of protein levels with DC Protein Assay Kit II (Bio-Rad), tissue homogenates were diluted to $5 \mathrm{mg} / \mathrm{ml}$ protein and IL-6 levels per mg heart homogenate were determined with mouse IL-6 ELISA Ready-setgo (eBiosciences).

\section{Atherosclerosis: tissue processing, histology, and morphometry}

At the end of the experimental period, at 29 weeks of age, mice were anaesthetized with pentobarbital (100 mg/kg, i.p.). Blood was taken in EDTA via cardiac puncture, followed by perfusion of the arterial tree under physiological pressure with PBS containing sodium nitroprusside (Sigma-Aldrich) and subsequently by $1 \%$ paraformaldehyde. The aortic arch including its main branch points was removed, fixed overnight in $1 \%$ paraformaldehyde, longitudinally embedded in paraffin, and sectioned. Twenty consecutive sections $(4 \mu \mathrm{m})$ representing the central area of the aortic arch with an intact morphology of the arch and branch points were selected. For histological analysis of atherosclerosis, 4 sections (20 $\mu \mathrm{m}$ apart) were stained with hematoxylin and eosin (H\&E) and the atherosclerotic lesions were classified as "initial" or "advanced" according to the Virmani classification (36). Plaque area was determined using a Leica DM3000 light microscope and a 10/0.3 NA on 20/0.5 objective (Leica Microsystems) coupled to a computerized morphometry system (Qwin 3.5; Leica, Wetzlar, Germany). Images were captured using a Leica DFC 425c camera. Morphometric parameters were determined as described previously (37). 


\section{Atherosclerosis: flow cytometry}

Blood and spleen were collected, processed and fluorescently stained with either antibody mix 1 (CD3-FITC, CD4-PerCp, CD8-eFLUO450, CD25-APC, Foxp3$\mathrm{PE}$ and B220-PE-Cy7) or antibody mix 2 (CD11b-PE-Cy7, Ly6C-APC, Ly6G-PE, CD3-FITC, B220-APC-eFLUO780 and NK1.1-PerCp-Cy5.5) or isotype control IgG (all BD Biosciences or eBioscience). Cells were analysed using a FACS-Canto II (BD Biosciences) flowcytometer.

\section{Atherosclerosis: (immuno) histochemistry}

Aortic arch sections were immunolabeled with anti-Mac3 monoclonal antibody (mAb) (1:30; BD Biosciences PharMingen) to detect macrophages, anti-CD3 (1:200; Dako) to detect $\mathrm{T}$ lymphocytes and anti-CD45 $\mathrm{mAb}$ (1:5000; $\mathrm{BD}$ Biosciences) to detect leukocytes. Antibody staining was visualized by diaminobenzidine or Vectastain red. Sirius red staining was performed for analysis of collagen content.

\section{Atherosclerosis: analysis of cytokine and cholesterol levels}

Enzyme-linked immunosorbent assays (ELISAs) were used to quantify concentrations of cytokines IL-6, IL-10 and MCP-1 (all R\&D systems) in plasma. Cholesterol levels in plasma were measured using the Cholesterol CHOD-PAP method (Roche).

\section{Statistical Analysis}

Data analysis was performed with GraphPad Prism 5. Data were presented as mean +/SEM. Differences between groups were tested using One-way ANOVA and Dunnett's multiple comparison post hoc test. $\mathrm{P}<0.05$ was considered statistically significant. 


\section{Results}

Expression and purification of recombinant $h(S 360 A)-P C$

hwt-PC and hS360A-PC were expressed under serum-free conditions at levels of 5 $\mathrm{mg} /$ liter and purified with overall recoveries of $\sim 40 \%$. SDS-PAGE gel analysis under reducing conditions, revealed the presence of the light chain as well as the $\alpha, \beta$ and $\gamma$ isoforms of the heavy chain (Figure 1). Upon activation by Protac, a fast-acting PC activator isolated from the venom of the copperhead snake Agkistrodon contortrix, the heavy chain isoforms shifted to a slightly lower migration position, consistent with full activation (95\%) as judged by SDS-PAGE (Figure 1). The purified hS360AAPC preparation contained no measurable $(<0.03 \%)$ amidolytic activity toward the chromogenic substrate S-2366.

\section{bwt- and hS360A-(A)PC reduce infarct area in a myocardial $I / R$ mouse model}

To examine the effects of APC treatment on myocardial I/R injury, mice were subjected to 1 hour ischemia by ligation of a suture around the LAD artery, followed by 2 hours of reperfusion. Mice received a first dose $(0.4 \mathrm{mg} / \mathrm{kg})$ of hwt-PC, hS360A-PC, hwtAPC, hS360A-APC or placebo (saline) 15 minutes after induction of ischemia and a second dose of the same treatment 5 minutes after induction of reperfusion. Next, infarct areas in the different groups were determined by Evans Blue/TTC staining (Figure 2A). Figure 2B shows that the infarct area in the placebo-treated mice was 26.1\%. Remarkably, hwt-PC, hS360A-PC, hwt-APC and hS360A-APC treatment all significantly reduced myocardial infarct area $(\mathrm{P}<0.05)$ to values of $11.1 \%$ to $14.3 \%$.

bwt- and hS360A-(A)PC have no effect on IL-6 levels in a myocardial I/ $\mathrm{R}$ mouse model

Interleukin 6 (IL-6) is a cytokine with pro-inflammatory and pro-coagulant properties that stimulates hepatocytes to produce several acute-phase proteins, like $\mathrm{C}$ reactive protein. Increased levels of IL-6 were found in coronary heart disease patients and were associated with prognosis (38). We measured IL-6 levels in tissue homogenates of heart tissue from mice that underwent myocardial I/R. We observed no significant difference in IL-6 levels between mice that received placebo and mice in the different $\mathrm{h}(\mathrm{A}) \mathrm{PC}$ treatment groups (Figure 2C). 
A

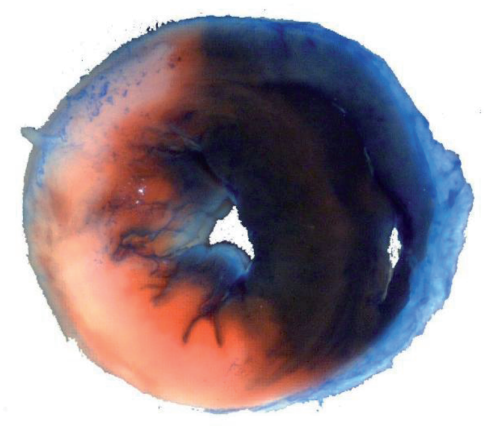

B
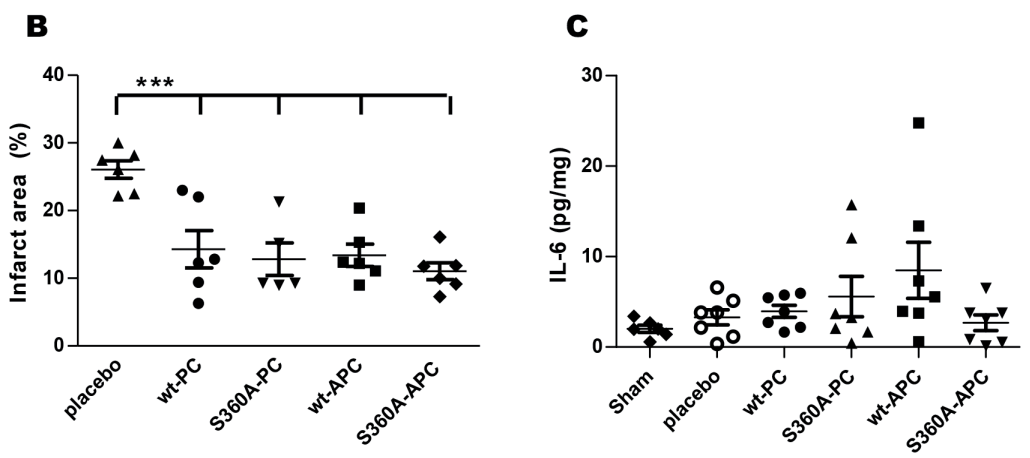

\section{Figure 2. Treatment with wt- or S360A-(A)PC decreases infarct area}

Mice were subjected to $1 \mathrm{~h}$ ischemia and $2 \mathrm{~h}$ reperfusion. Treatment $(0.4 \mathrm{mg} / \mathrm{kg}$ ) or placebo (saline) were administered i.v. 15 min after induction of ischemia and a second time 5 minutes after induction of reperfusion. A) Representative picture of the Evans Blue/TTC staining after $2 \mathrm{~h}$ reperfusion. The normal tissue (non infarcted area) is stained dark blue, whereas the ischemic area (area at risk (AAR)) is stained brick red and the non-viable infarct area (area of infarction (AOI)) did not stain and remained pale. B) Infarct area (percentage AOI of AAR) as determined by Evans Blue/TTC staining. C) IL-6 concentrations were determined in heart tissue homogenates $(5 \mathrm{mg} / \mathrm{ml}$ protein content) by ELISA and IL-6 per mg heart homogenate was calculated. All graphs show mean +/- SEM, statistical significance was tested using oneway analysis of variance with Dunnett post hoc test, ${ }^{* * *} \mathrm{P}<0.001$.

\section{bwt- and hS360A-(A)PC do not influence plaque area in Apo $\mathrm{E}^{-/-}$mice}

Having observed that all hPC and hAPC forms conferred cytoprotection in the acute inflammatory myocardial I/R model, we subsequently investigated the effect of hAPC administration on plaque development in a chronic inflammatory mouse model of atherosclerosis. ApoE ${ }^{-/-}$mice were treated twice weekly with placebo, or hwt-PC, hS360A-PC, hwt-APC or hS360A-APC (0.2 mg/kg) for 8 weeks, after which atherosclerotic plaque development in the aortic arch was determined. Representative images of hematoxylin and eosin ( $\mathrm{H} \& \mathrm{E})$ stained initial and advanced plaques are shown in Figure 3A-B. On average, we detected 1 initial plaque and 3 advanced plaques in 
the mice from all groups analyzed (Figure 3C). We did observe a (non-significant) reduction in the total number of plaques for the hS360A-PC group. The number of advanced plaques was greatest in the placebo group and slightly decreased in the groups receiving treatment. Surprisingly, in the four treatment groups, total plaque area was not significantly different from the placebo group (Figure 3D). The areas of the individual initial plaques were comparable between groups (Figure 3E), with the placebo, hS360APC and hS360A-APC groups presenting with the lowest inter-individual variation.
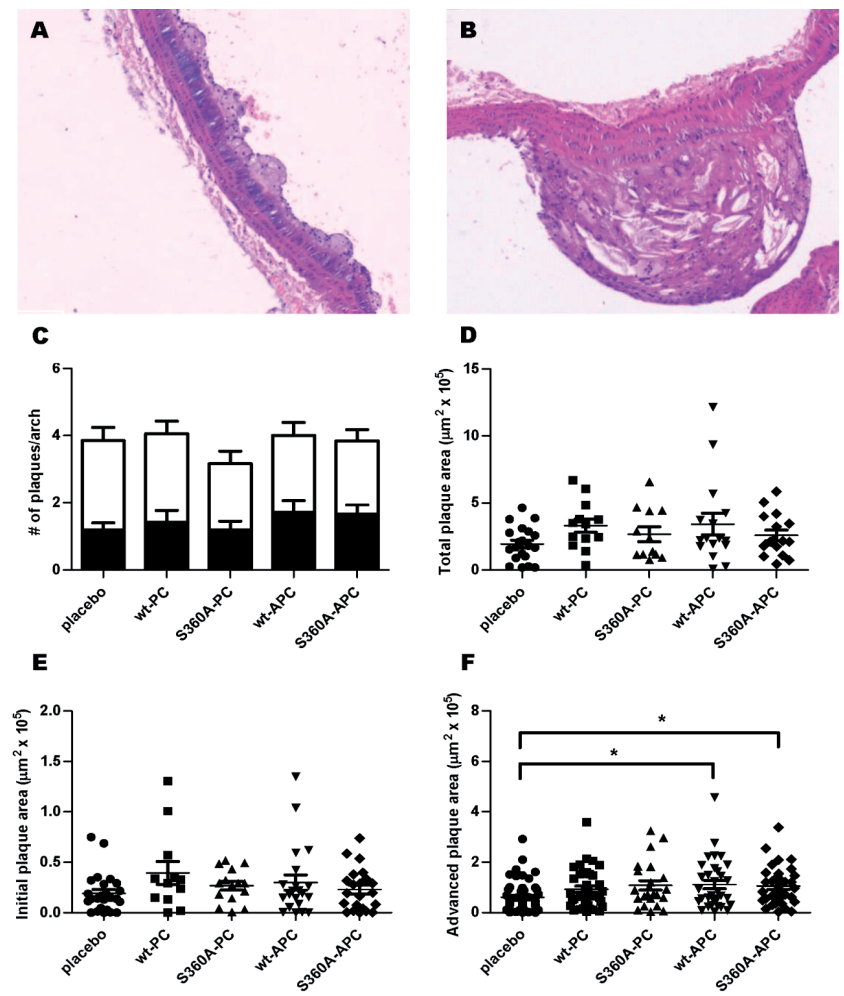

Figure 3. Treatment with wt- or S360A-(A)PC does not alter plaque area or \# of plaques

$\mathrm{ApoE}^{-/}$mice were injected with saline or $0.2 \mathrm{mg} / \mathrm{kg}$ of wt-PC, S360A-PC, wt-APC or S360A-APC. Images of an initial (A) and an advanced (B) plaque after hematoxylin and eosin (H\&E) staining. C) Atherosclerotic lesions were classified as initial (black bars) or advanced (white bars) and numbers of both type of plaques per mouse were determined. D) Total plaque area in the aortic arch as determined by H\&E staining. E) Surface area of individual initial plaques. F) Surface area of individual advanced plaques. All graphs show mean + /- SEM, statistical significance was tested using one-way analysis of variance with Dunnett post hoc test, ${ }^{*} \mathrm{P}<0.05$.

Surprisingly, the areas of individual advanced plaques were significantly increased in the hwt-APC and hS360A-APC groups compared to the placebo group (Figure 3F), although the data points of plaque sizes in the five different groups show considerable overlap. So, overall no clear effect of hwt- or hS360A-(A)PC on plaque development was observed. 


\section{Effect of $h(A) P C$ variants on plaque phenotype and the immune system}

Subsequently the plaque phenotype was further characterized. Collagen content (Figure $4 \mathrm{~A} / \mathrm{E}$ ) and infiltration of macrophages (Figure 4B/F), leukocytes (Figure 4C/G) and $\mathrm{T}$ cells (Figure $4 \mathrm{D} / \mathrm{H}$ ) were determined in all plaques. hS360A-APC treated mice had, compared to placebo treated mice, significantly lower collagen content in their plaques $(\mathrm{P}<0.05)$, while they had increased leukocyte $(\mathrm{P}<0.01)$ and $\mathrm{T}$ cell $(\mathrm{P}<0.01)$ infiltration. Interestingly, also the hwt-APC treated group presented with significantly lower collagen content in their plaques $(\mathrm{P}<0.05)$. In the hwt-PC and hwt-APC groups, we noted a trend towards increased $\mathrm{T}$ cell and leukocyte counts. Infiltration of macrophages in plaques had a low variation and was not different between the placebo and different treatment groups. So, the plaque phenotype was slightly changed by hS360A-APC treatment and not by other treatments.
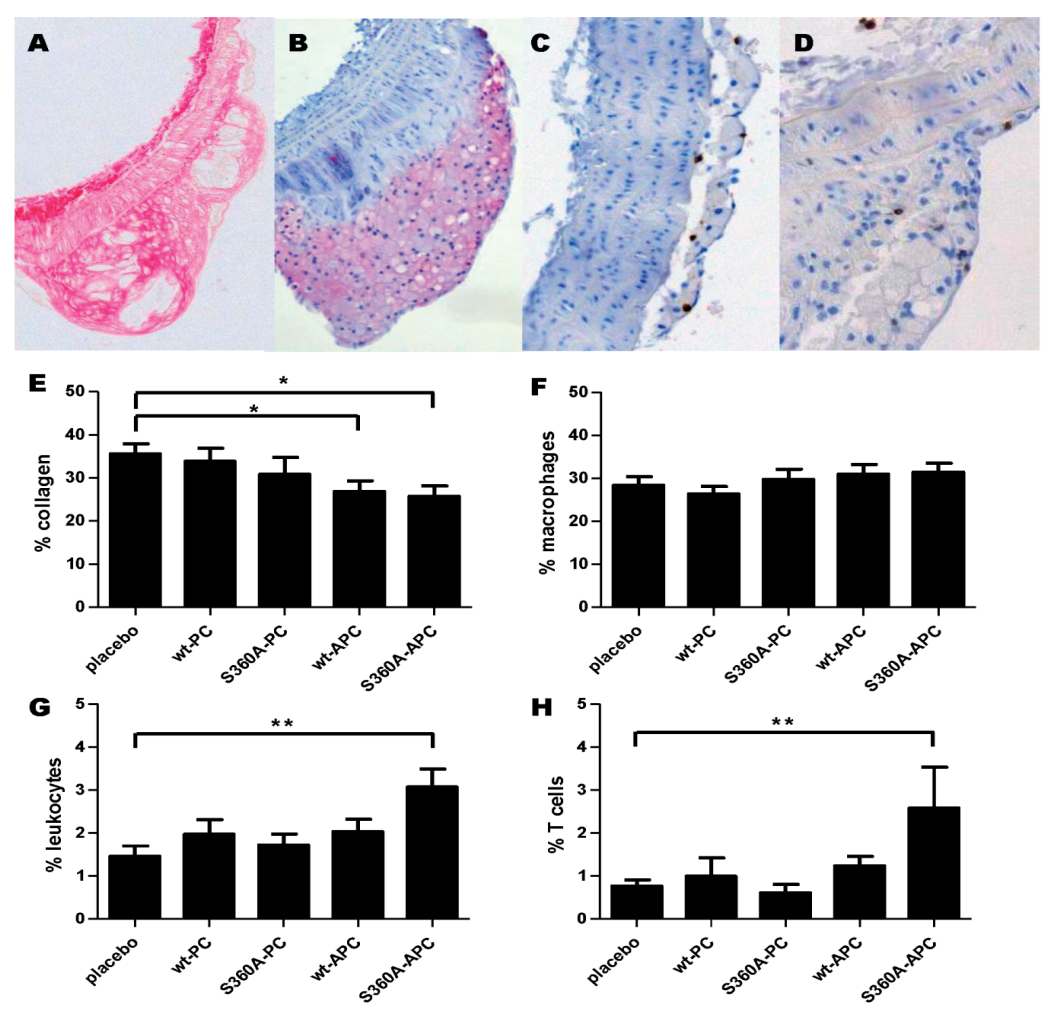

Figure 4. Only S360A-APC treatment influences plaque phenotype

Images of plaques stained for collagen (A), macrophages (B), leukocytes (C), and T cells (D). E) After sirius red staining, the percentage of collagen was determined by dividing the area of collagen by the total plaque area. F) After Mac-3 staining, the percentage of macrophages as a percentage of the total number of cells per plaque was calculated. G) After CD45 staining, the percentage of leukocytes as a percentage of the total number of cells per plaque was calculated. $\mathbf{H}$ ) After $\mathrm{CD} 3$ staining, the percentage of $\mathrm{T}$ cells of the total number of cells per plaque was calculated. All graphs show mean $+/$ - SEM, statistical significance was tested using one-way analysis of variance with Dunnett post hoc test, ${ }^{*} \mathrm{P}<0.05$, ${ }^{* *} \mathrm{P}<0.01$. 
The effects of hAPC treatment on the innate and adaptive immune system were assessed by flow cytometric analysis of immune cells isolated from both blood and spleen. No significant differences were observed between quantities of B cells, granulocytes, NK cells, NKT cells and different types of monocytes $\left(\mathrm{Ly} 6 \mathrm{C}^{\text {high/low/ } /}\right)$ and $\mathrm{T}$ cells $\left(\mathrm{CD}^{+}\right.$, $\mathrm{CD} 8^{+}, \mathrm{CD} 25^{+}$and Foxp3 $3^{+}$) (Figure 5-7).

A
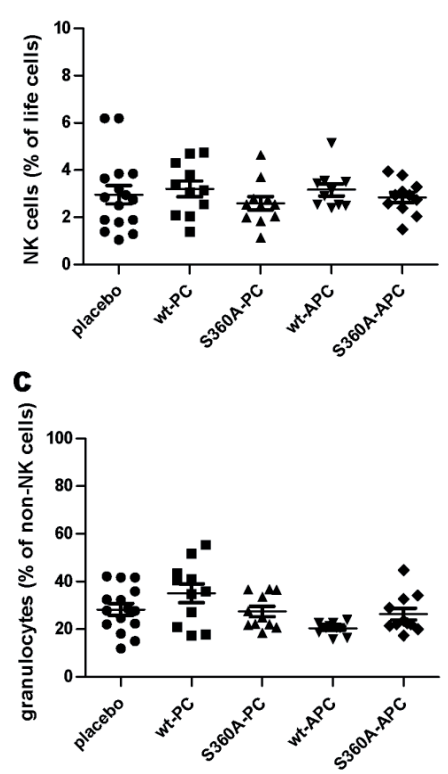

E

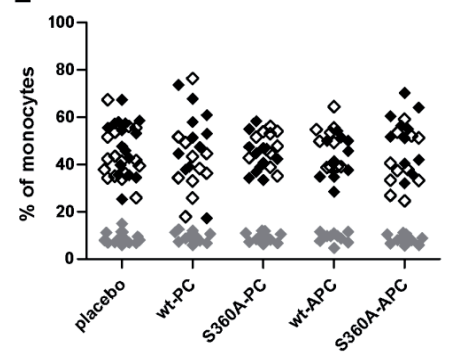

B

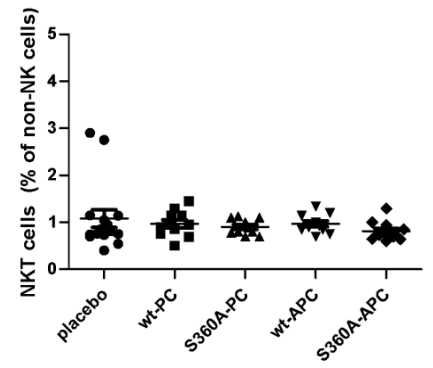

D

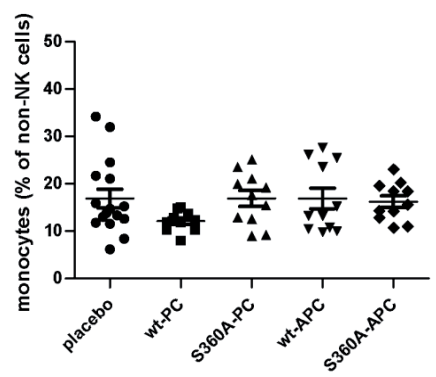

Figure 5. Effect of wt- and S360A-(A)PC treatment on immune cells in blood

Percentages of A) NK cells (NK1-1+, CD3-), B) NKT cells (NK1-1+, CD3+), C) Granulocytes (CD11b+, Ly6G+) and D) monocytes (CD11b+, Ly6G-) were determined by flow cytometric analysis of mice blood. E) Monocytes were further characterized as Ly6C high $(\checkmark)$, Ly6C low $(\downarrow)$ or Ly6C- $(\diamond)$. Graphs A-D show mean +/- SEM. 
A

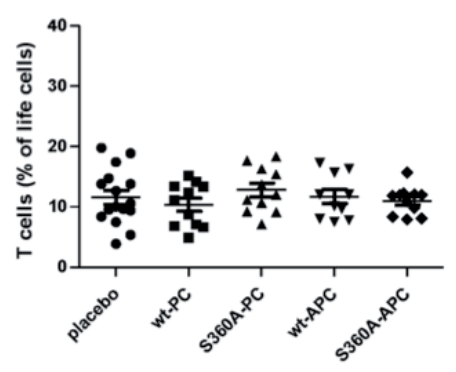

C

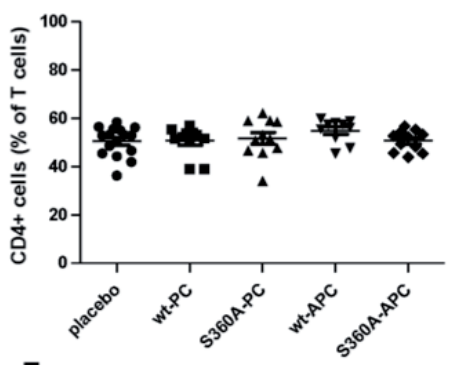

E

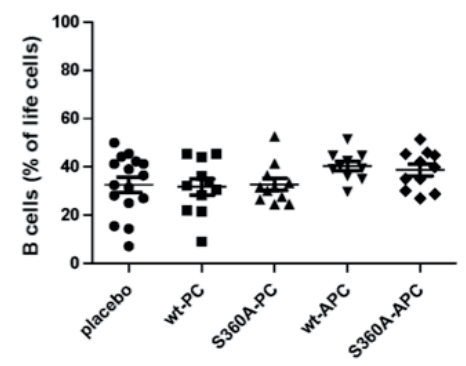

B

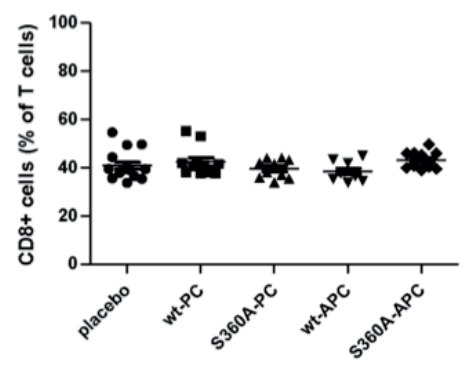

D

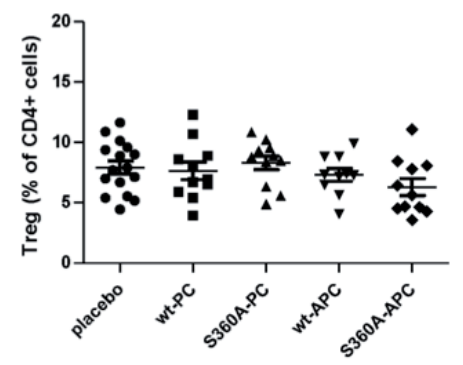

Figure 6. Effect of wt- and S360A-(A)PC treatment on immune cells in blood

Percentages of A) total T cells (CD3+), B) CD8+ T cells, C) CD4+ T cells, D) regulatory T cells (FOXP3+, $\mathrm{CD} 25+)$ and E) B cells (B220+) were determined by flow cytometric analysis of mice blood. All graphs show mean + /- SEM. 
A

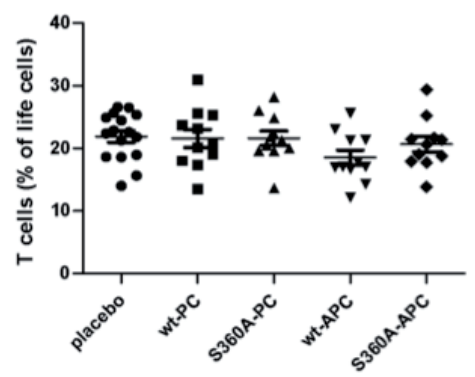

C

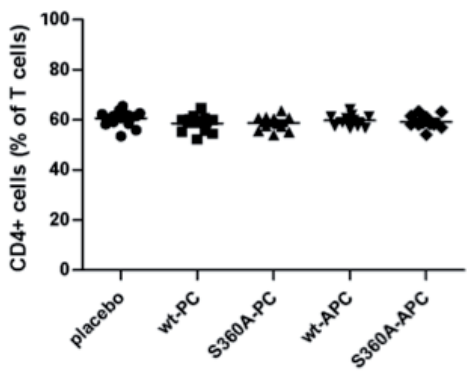

$\mathbf{E}$

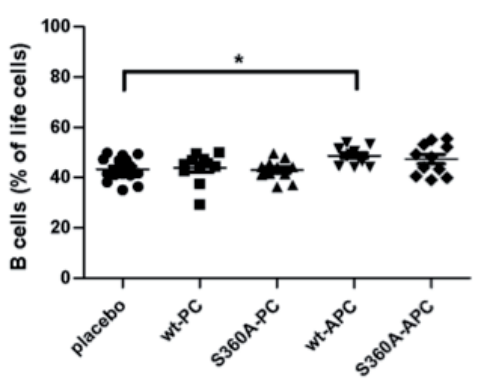

B

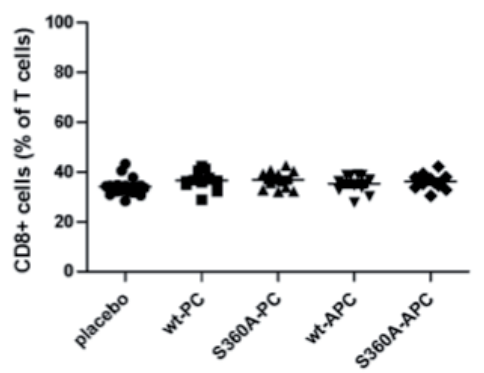

D

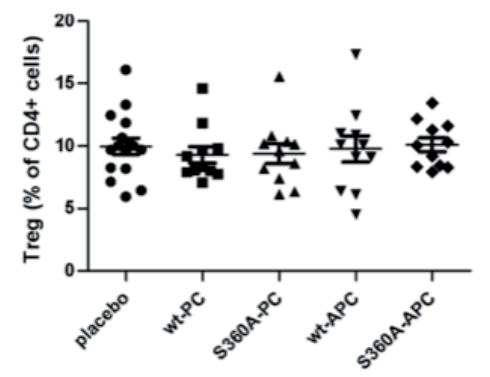

Figure 7. Effect of wt- and S360A-(A)PC treatment on immune cells isolated from spleen

Percentages of $\mathbf{A}$ ) total T cells (CD3+), B) CD8+ T cells, C) CD4+ T cells, D) regulatory T cells (FOXP3+, CD25+) and E) B cells (B220+) were determined by flow cytometric analysis of cells isolated from mice spleen tissue. All graphs show mean +/- SEM, statistical significance was tested using one-way analysis of variance with Dunnett post hoc test, $* \mathrm{P}<0.05$.

Effect of $h(A) P C$ variants on plasma levels of cytokines IL-6, IL-10 and MCP-1

We next determined the levels of cytokines IL-6, IL-10 and MCP-1 and of cholesterol in the plasma from the animals included in this study and to this end prepared plasma pools by mixing equal volumes of plasma for each of the animals studied per group. Mice treated with hS360A- PC had significantly lower IL-6 levels $(\mathrm{P}<0.001)$ and increased MCP-1 levels $(\mathrm{P}<0.05)$, see Figure 8A/C. In the hwt-APC group, the IL-6 levels were also lower than in the placebo group $(\mathrm{P}<0.01)$. The latter group was highest 
of all groups determined here, for both the IL- 6 and the IL-10 measurements. A nonsignificant trend was observed for the lowering effect of hS360A-PC on IL-10 levels (Figure 8B). Cholesterol levels were slightly but significantly higher in mice treated with hwt-PC, hS360A-PC and hS360A-APC compared to placebo treated mice (Figure 8D).

A

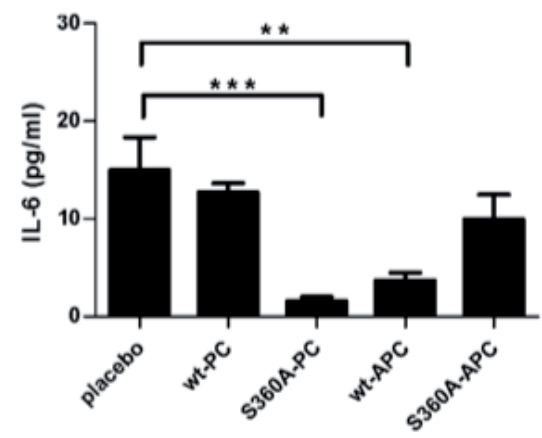

C

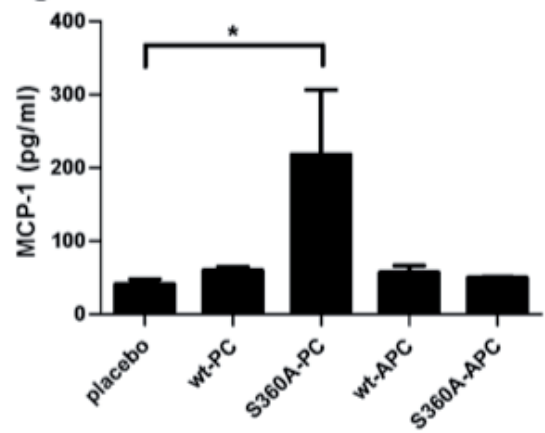

B

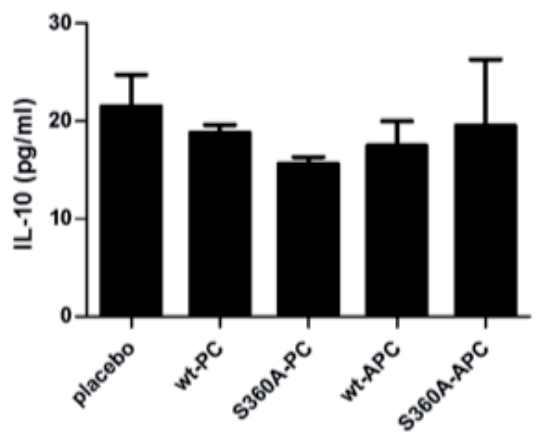

D

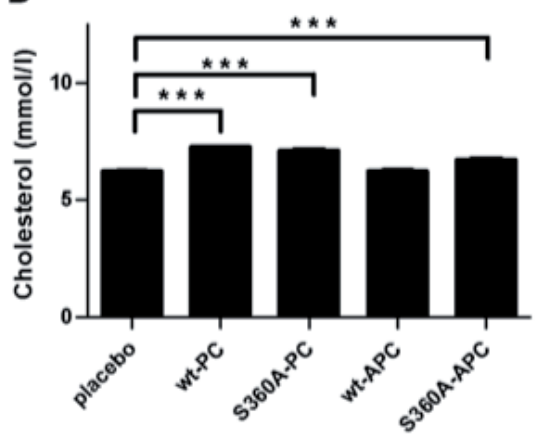

Figure 8. Effects of wt- and S360A-(A)PC treatment on cytokine and cholesterol levels

A) Interleukin (IL) 6 levels measured with ELISA in mouse plasma pools. B) IL-10 levels measured with ELISA in mouse plasma pools. C) Monocyte chemotactic protein-1 (MCP-1) levels measured with ELISA in mouse plasma pools. D) Cholesterol levels in mouse plasma pools measured with cholesterol CHODPAP method. All graphs show mean +/- SEM, statistical significance was tested using one-way analysis of variance with Dunnett post hoc test, $* \mathrm{P}<0.05$, ** $\mathrm{P}<0.01$, *** $\mathrm{P}<0.001$. 


\section{Discussion}

Several elegant studies have previously provided experimental evidence that wt-APC protects against acute myocardial I/R injury via both anti-inflammatory and antiapoptotic effects (Table 1). In this study we show that not only hwt-APC, but also hwtPC, hS360A-PC and hS360A-APC significantly reduce infarct area in a myocardial I/R model. This finding, which is a phenomenological observation at this point, deserves further mechanistic explanation. It has been shown $(39,40,41)$ that EPCR and PAR1 are associated with caveolin-1 within endothelial lipid rafts. When EPCR is bound to the gamma-carboxyglutamic (Gla)-domain of either wt-(A)PC or S360A-(A)PC, it dissociates from caveolin-1 and alters the G-protein mediated signaling of PAR-1 from pro-inflammatory $\left(G_{\mathrm{q}}\right.$ and/or $\left.G_{12 / 13}\right)$ to anti-inflammatory signaling (pertussis toxin-sensitive $G_{i}$-protein). In this caveolin-unbound state, cleavage of PAR-1 by thrombin or APC and resulting $G_{i}$ activation, initiates a barrier protective and antiinflammatory response, which includes activation of Rac1 and inhibition of RhoA and NFKB signaling cascades. We speculate that these mechanisms likely contributed to the protective effects observed for the hPC and hAPC forms tested by us, including the proteolytically inactive hS360A-(A)PC variant. Despite the mutation of the active site, S360A-(A)PC is still able to bind EPCR via its Gla-domain $(42,43)$ and can thereby influence PAR-1 signaling pathways.

Additionally it was shown that both PC and APC significantly decrease LPS-induced reactive oxygen species (ROS) generation by macrophages. Remarkably these effects were EPCR-independent and only in APC-treated cells accompanied by inhibited NFKB activation (44). Alternatively, these antioxidant effects of (A)PC may be related to inhibition of NADPH oxidase or by regulation of transcription factor Sp1 (45). PC and APC reduced copper-induced LDL peroxidation via interaction of their Gla-domains with phospholipids on the surface of LDL particles, and protected unsaturated fatty acids against oxidation (44). Both increased ROS generation and lipid peroxidation are observed during $\mathrm{I} / \mathrm{R}$ injury and have been described to play an important role in the pathophysiology of myocardial injury $(46,47)$. The observation that also zymogen PC provides in vitro antioxidant activity implies that the antioxidant properties are at least partly independent of the catalytic side and in line with our finding that both hwt- and hS360A-(A)PC have protective properties in acute myocardial I/ $\mathrm{R}$ injury. There are several possible explanations for the observation that zymogen PC induced a similar reduction in infarct size as APC, while in fact its antioxidant capacities are those of APC (44). Firstly, activation of PC on endothelial cells generates APC with 4-fold higher cytoprotective barrier protective properties than exogenous APC (42). This discrepancy cannot be explained by differences in the local APC concentrations and possibly originates because protective signaling by APC is mechanistically linked to PC activation (42). Additionally, both zymogen PC and S360A-(A)PC have the advantage that these are not inhibited by circulating serpins and therefore have a longer half life 


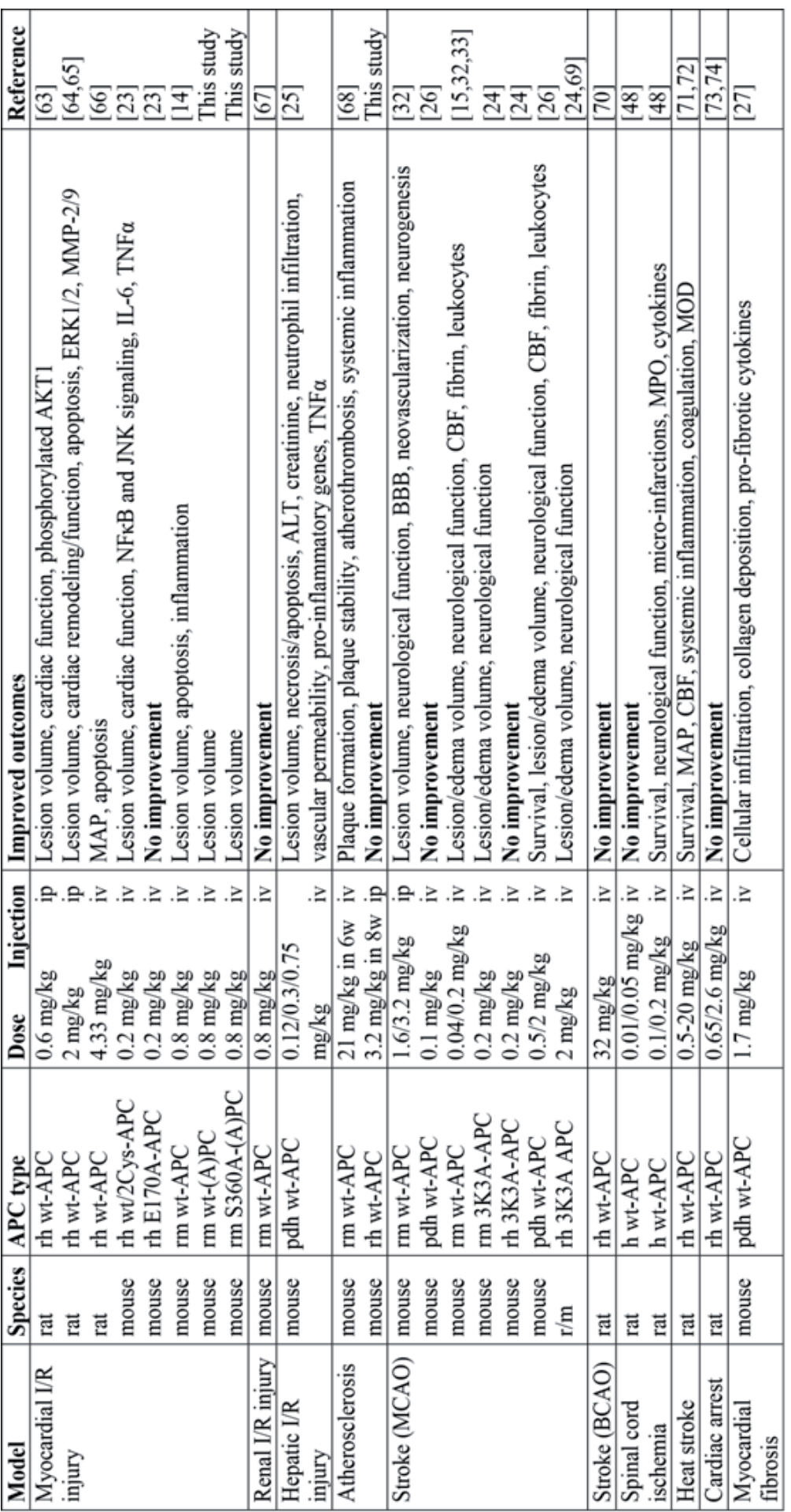

苛

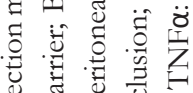

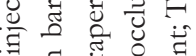

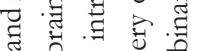

司 䒕

ए $0 . \hat{1}$ 可

苛苛

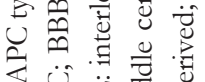

《ن் 吾

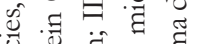

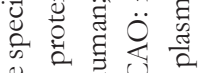

छ च छ

它谣的

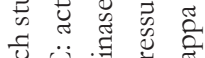

प्ठ

tे $<$. ज्ञ

I

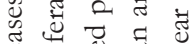

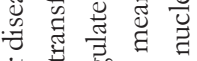

壱

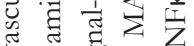

๗்

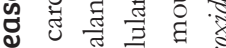

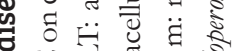

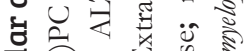

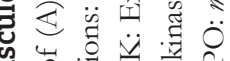

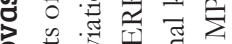

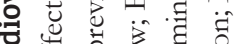

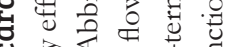

I

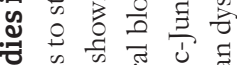

岁苛苛苛

U ¿

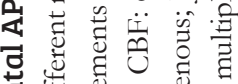

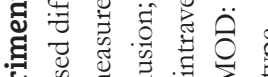

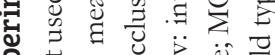

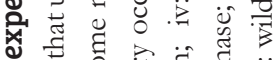

उ के ठैं

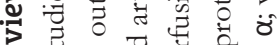

峁

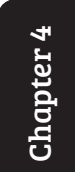

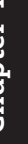

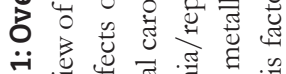

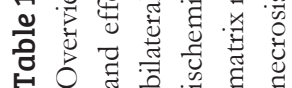


in vivo (17), which would make these proteins better suited for potential application in a clinical therapeutic context, like for organ/tissue protection. Additional beneficial properties can be attributed to S360A-PC, since this molecule, unlike its wild-type homolog, is unlikely to result in bleeding side effects after in vivo thrombomodulinmediated activation by thrombin.

Although S360A-(A)PC still has residual anticoagulant activity because of its ability to limit thrombin formation through competition with FXa and FIXa for resp. FVa and FVIIIa, it is unlikely that these anticoagulant properties of S360A-(A)PC have contributed to the reduced myocardial infarct area observed in S360A-(A)PC treated mice. It has been shown before $(14,48)$ that the use of in vivo thrombin inhibition by heparin administration or the selective thrombin generation inhibitor dansyl glutamylglycyl-arginyl chloromethyl ketone-treated activated factor X (DEGR-FXa), failed to reduce myocardial I/R injury and I/R-induced spinal cord injury. Likewise, another study by Wang and coworkers (49) compared the effects of the cytoprotective-selective 5A-APC variant $(<10 \%$ anticoagulant but normal cytoprotective activity) and the anticoagulant-selective E149A-APC variant ( $>3$-fold increased anticoagulant activity but defective cytoprotective activities) in a murine focal ischemic stroke model. Despite its reduced anticoagulant activity, 5A-APC significantly decreased infarct- and edema volume and improved neurological outcome, while E149A-APC administration resulted in significantly worsened neurological outcome and increased infarct- and edema volume. Additionally, E149A-APC treatment was associated with an increased risk of bleeding as indicated by 5 -fold increased hemoglobin levels in the ischemic brain.

In contrast to the study of Loubele (14) and coworkers we did not find a significant effect of h(A)PC treatment on IL-6 levels in heart homogenates after acute myocardial $\mathrm{I} / \mathrm{R}$ injury. One possible explanation for the difference is the fact that IL-6 levels in placebo treated mice were 5 -fold lower in the present study, leaving less room for a further decrease by h(A)PC treatment. The discrepancy may also arise from the fact that we used $\mathrm{h}(\mathrm{A}) \mathrm{PC}$ and Loubele and co-workers mAPC. Previous research has shown that hAPC was significantly less potent in murine stroke models as compared to mAPC (24). The observed difference cannot be explained by a difference in proteolytic activity, but the lower affinity of hAPC for mPAR-1 than for the human isoform of this receptor probably plays a role (50). Differences in sequence $(69 \%$ sequence identity between hAPC and mAPC (22)) or post-translational modifications can possibly explain these different affinities.

While we hypothesized that administration of $\mathrm{h}(\mathrm{A}) \mathrm{PC}$ would also influence plaque development in the long term chronic atherosclerosis mouse model, we found that none of the h(A)PC variants significantly protected against the development of atherosclerosis in this mouse model of chronic inflammation. hwt-APC and hS360AAPC even slightly increased plaque area of advanced plaques, but the differences between these groups and the other groups were relatively small. Probably the lack 
of effect of h(A)PC on reducing plaque development can be explained by the in vivo bioavailability after injection. Previously it was shown that i.p. administration of 0.1 or $0.8 \mathrm{mg} / \mathrm{kg} \mathrm{mAPC}$ produces a transient rise in APC levels in circulation for 3 hours, with a plateau after around $20 \mathrm{~min}(32,51)$. This transient rise in (A)PC concentration in circulation, that was achieved twice a week in our model, was apparently not enough to initiate protective signaling that lasted long enough to provide long term protection against atherosclerosis development during the time span of our study.

We have however observed a clear effect of hS360A-PC and hwt-APC administration on IL-6 levels in plasma. The decrease in concentration of the pro-inflammatory cytokine IL- 6 after hS360A-PC and hwt-APC treatment can possibly be explained by inhibited IL-6 release by neutrophils as a result of h(A)PC treatment (52). Conflicting reports on the role of IL-6 in the development of murine atherosclerosis have appeared; some studies show that IL-6 promotes atherosclerosis while other studies show that IL- $-6^{--}$mice are more atherogenic than control mice, so it is not clear what the exact contribution of IL-6 to atherogenesis is (53-58). In hS360A-PC treated mice we found an increased level of MCP-1. Remarkably, this was not accompanied by increased percentages of leukocytes and macrophages in plaques and therefore likely had no effects on plaque development. In hS360A-APC treated mice the phenotype of the atherosclerotic plaques was slightly changed. Plaques contained significantly less collagen, while the infiltration of both leukocytes and T-cells was increased. Both an increase in activated immune cells and a decrease of collagen in plaques are associated with a more unstable plaque phenotype (59, 60).

Analysis of other cardiovascular studies which have studied the in vivo effects of (A) PC in a cardiovascular context (Table 1) reveals that administration of APC has mainly been studied in models of acute diseases like I/R injury and stroke. In most of these experimental models administration of APC improved primary and secondary outcome variables, like survival, lesion/edema volume, cardiac/neurological function and lowered the concentration of inflammation markers. For comparison between experiments it is important to realize that administration of $0.2 \mathrm{mg} / \mathrm{kg}$ APC i.v. and $0.8 \mathrm{mg} / \mathrm{kg}$ APC i.p. result in similar APC plasma pharmacokinetic profiles (32). Wang et al. (23) show that an APC mutant that is not able to cleave PAR-1 (E170A-PC) does not have any protective effect, while an APC mutant with greatly impaired anticoagulant function (3K3A-APC) was as effective as wt-APC in decreasing lesion volume and improving neurological function. This result in itself suggests that mainly the cytoprotective PAR-1-dependent effects of APC, rather than the anticoagulant functions of APC, are important for protection in cardiovascular disease. However, the current study showed that hS360AAPC was as effective as hwt-APC in decreasing lesion volume in myocardial I/R injury, although this variant had strongly decreased anti-coagulant activity and was not able to cleave PAR-1. We hypothesize that hS360A-APC was protective because it can bind through its Gla-domain to EPCR and thereby promotes protective PAR-1 signaling 
via endogenous mAPC and thrombin (21). Both these serine proteases are known to be formed during I/R damage $(61,62)$. Alternatively, hS360A APC may reduce ROS formation and bind through its Gla-domain to phospholipids on LDL particles, resulting in reduced lipid peroxidation as discussed above for PC and APC. Both effects can contribute to decreased lesion volume in acute myocardial I/R injury.

We have studied the effects of 4 different $\mathrm{h}(\mathrm{A}) \mathrm{PC}$ variants in mouse models for cardiovascular disease: in an acute I/R injury model and in a chronic atherosclerosis model. We have shown that treatment with both hwt and hS360A-(A)PC decreases infarct area in our acute murine myocardial I/R injury model. However, no significant effects were observed for the development of atherosclerotic plaques, a chronic slow process. Trends were observed that indicate that $\mathrm{h}(\mathrm{A}) \mathrm{PC}$ may be able to reduce in particular IL-6 and collagen levels, but under the conditions used in our experiments these did not lead to significant differences in total plaque area or plaque phenotype. Comparison of our data with those in literature learns that the (A)PC dose, mode of administration and species of administered (A)PC varied among different studies. We therefore propose that for future experiments in chronic disease models using mice, it will be preferable to include at least $\mathrm{mAPC}$, which is administered preferably intravenously and more frequently than in the current study. If possible a slow-release device would be a preferred method, but this will require that the (A)PC preparations are stable for extended periods of time at body temperature. Nonetheless, while recognizing that such controlled study has not been provided yet, we regard our current study as valuable information that will enable the needed further study. In conclusion, this study showed that both hwt and hS360A-(A)PC protect in an acute murine myocardial I/R injury model and have the potential to influence development of chronic inflammation as occurring during atherosclerosis as well.

\section{Acknowledgements}

We want to thank Sarah T.B.G. Loubele and Peter Leenders (Cardiovascular Research Institute Maastricht, Maastricht University, The Netherlands) for assistance with the acute myocardial I/R experiments and Björn Dahlbäck (Lund University, Malmö, Sweden) for providing proteins. This work was supported by Grant 125/2008/OPI from the Netherlands Thrombosis Foundation (to H.t.C) and by the Cardiovascular Research Institute Maastricht (to G. A. F. N.) 


\section{References}

1. Stearns-Kurosawa D, Kurosawa S, Mollica J, Ferrell G, Esmon C (1996) The endothelial cell protein C receptor augments protein $\mathrm{C}$ activation by the thrombin-thrombomodulin complex. PNAS 96: 1021210216.

2. Kalafatis M, Rand M, Mann K (1994) The mechanism of inactivation of human factor V and human factor Va by activated protein C. J Biol Chem 269: 31869-31880.

3. Fay P, Smudzin T, Walker F (1991) Activated protein C-catalyzed inactivation of human factor VIII and factor VIIIa. Identification of cleavage sites and correlation of proteolysis with cofactor activity. J Biol Chem 266: 20139-20145.

4. Nicolaes G, Dahlbäck B (2002) Factor V and thrombotic disease: description of a janus-faced protein. Arterioscler Thromb Vasc Biol 22: 530-538.

5. Nicolaes G, Dahlbäck B (2003) Congenital and acquired activated protein C resistance. Semin Vasc Med 3: 33-46.

6. Broekmans AW, Veltkamp JJ, Bertina RM (1983) Congenital protein C deficiency and venous thromboembolism. A study of three Dutch families. N Engl J Med 309: 340-344.

7. Griffin JH, Evatt B, Zimmerman TS, Kleiss AJ, Widerman C (1981) Deficiency of protein C in congenital thrombotic disease. J Clin Invest 68: 1370-1373.

8. Marlar RA, Montgomery RR, Broekmans AW (1989) Diagnosis and treatment of homozygous protein C deficiency. Report of the Working Party on Homozygous Protein C Deficiency of the Subcommittee on Protein C and Protein S, International Committee on Thrombosis and Haemostasis. J Pediatr 114: 528-534.

9. Seligsohn U, Berger A, Abend M, Rubin L, Attias D, et al. (1984) Homozygous protein C deficiency manifested by massive venous thrombosis in the newborn. N Engl J Med 310: 559-562.

10. Bae J, Yang L, Rezaie A (2007) Receptors of the protein C activation and activated protein C signaling pathways are colocalized in lipid rafts of endothelial cells. Proc Natl Acad Sci USA 104: 2867-2872.

11. Mosnier LO, Zlokovic BV, Griffin JH (2007) The cytoprotective protein C pathway. Blood 109: 31613172.

12. Murakami K, Okajima K, Uchiba M, Johno M, Nakagaki T, et al. (1996) Activated protein C attenuates endotoxin-induced pulmonary vascular injury by inhibiting activated leukocytes in rats. Blood 87: 642647.

13. Zlokovic B, Zhang C, Liu D, Fernandez J, Griffin J, et al. (2005) Functional recovery after embolic stroke in rodents by activated protein C. Ann Neurol 58: 474-477.

14. Loubele ST, Spek CA, Leenders P, van Oerle R, Aberson HL, et al. (2009) Activated protein C protects against myocardial ischemia/reperfusion injury via inhibition of apoptosis and inflammation. Arterioscler Thromb Vasc Biol 29: 1087-1092.

15. Fernandez JA, Xu X, Liu D, Zlokovic BV, Griffin JH (2003) Recombinant murine activated protein C is neuroprotective in a murine ischemic stroke model. Blood Cells Mol Diseases 30: 271-276.

16. Taylor Fj, Chang A, Esmon C, D'Angelo A, Vigano-D'Angelo S, et al. (1987) Protein C prevents the coagulopathic and lethal effects of Escherichia coli infusion in the baboon. J Clin Invest 79: 918-925.

17. Gale AJ, Sun X, Heeb MJ, Griffin JH (1997) Nonenzymatic anticoagulant activity of the mutant serine protease Ser360Ala-activated protein C mediated by factor Va. Protein science 6: 132-140.

18. Nicolaes GAF, Bock PE, Segers K, Wildhagen KCAA, Dahlbäck B, et al. (2010) Inhibition of thrombin formation by active site mutated (S360A) activated protein C. J Biol Chem 285: 22890-22900. 
19. Mosnier LO, Gale AJ, Yegneswaran S, Griffin JH (2004) Activated protein C variants with normal cytoprotective but reduced anticoagulant activity. Blood 104: 1740-1744.

20. Ludeman M, Kataoka H, Srinivasan Y, Esmon N, Esmon C, et al. (2005) PAR1 cleavage and signaling in response to activated protein C and thrombin. J Biol Chem 280: 13122-13128.

21. Bae J, Yang L, Rezaie A (2008) Lipid raft localization regulates the cleavage specificity of protease activated receptor 1 in endothelial cells. J Thromb Haemost 6: 954-961.

22. Krisinger M, Guo LJ, Salvagno G, Guidi GC, Lippi G, et al. (2009) Mouse recombinant protein C variants with enhanced membrane affinity and hyper-anticoagulant activity in mouse plasma. FEBS J 276: 6586-6602.

23. Wang J, Yang L, Rezaie A, Li J (2011) Activated protein C protects against myocardial ischemic/ reperfusion injury through AMP-activated protein kinase signaling. J Thromb Haemost 9: 1308-1317.

24. Guo H, Wang Y, Singh I, Liu D, Fernandez JA, et al. (2009) Species-dependent neuroprotection by activated protein C mutants with reduced anticoagulant activity. J Neurochem 109: 116-124.

25. Park SW, Chen SW, Kim M, D'Agati VD, Lee HT (2009) Human activated protein C attenuates both hepatic and renal injury caused by hepatic ischemia and reperfusion injury in mice. Kidney Int 76: 739750.

26. Shibata M, Kumar SR, Amar A, Fernandez JA, Hofman F, et al. (2001) Anti-inflammatory, antithrombotic and neuroprotective effects of activated protein $\mathrm{C}$ in a murine model of focal ischemic stroke. Circulation 103: 1799-1805.

27. Sopel M, Rosin N, Falkenham A, Bezuhly M, Esmon C, et al. (2012) Treatment with activated protein $\mathrm{C}(\mathrm{aPC})$ is protective during the development of myocardial fibrosis: an angiotensin II infusion model in mice. PLoS One 7: e45663.

28. Yellon D, Hausenloy D (2007) Myocardial Reperfusion Injury. N Engl J Med 257: 1121-1135.

29. Libby P (2010) Inflammation in atherosclerosis. Arterioscler Thromb Vasc Biol 32: 2045-2051.

30. Sun YH, Shen L, Dahlbäck B (2003) Gla domain-mutated human protein C exhibiting enhanced anticoagulant activity and increased phospholipid binding. Blood 101: 2277-2284.

31. Friedrich U, Pötzsch B, Preissner K, Müller-Berghaus G, Ehrlich H (1994) Calcium-dependent activation of protein $\mathrm{C}$ by thrombin/thrombomudulin: role of negatively charged amino acids within the activation peptide of protein C. Thromb Haemost 72: 567-572.

32. Thiyagarajan M, Fernández J, Lane S, Griffin J, Zlokovic B (2008) Activated protein C promotes neovascularization and neurogenesis in postischemic brain via protease-activated receptor 1 . J Neurosci 28: 12788-12797.

33. Cheng T, Dong L, Griffin J, Fernandez J, Castellino F, et al. (2003) Activated protein C blocks p53mediated apoptosis in ischemic human brain endothelium and is neuroprotective. Nat Med 9: 338-342.

34. Jong W, Reitsma P, ten Cate H, de Winter R (2003) Modified two-step model for studying the inflammatory response during myocardial ischemia and reperfusion in mice. Comp Med 53: 522-526.

35. Erlich J, Boyle E, Labriola J, Kovacich J, Santucci R, et al. (2000) Inhibition of the tissue factorthrombin pathway limits infarct size after myocardial ischemia-reperfusion injury by reducing inflammation. Am J Pathol 157: 1849-1862.

36. Virmani R, Kolodgie F, Burke A, Farb A, Schwartz S (2000) Lessons from sudden coronary death: a comprehensive morphological classification scheme for atherosclerotic lesions. Arterioscler Thromb Vasc Biol 20: 1262-1275.

37. Donners M, Beckers L, Lievens D, Munnix I, Heemskerk J, et al. (2008) The CD40-TRAF6 axis is the key regulator of the CD40/CD40L system in neointima formation and arterial remodeling. Blood 111: 4596-4604.

38. Biasucci L, Vitelli A, Liuzzo G, Alltamura S, Caligiuri G, et al. (1996) Elevated Levels of Interleukin-6 in Unstable Angina. Circulation 94: 874-877. 
39. Bae JS, Rezaie AR (2009) Thrombin inhibits nuclear factor $\mathrm{kB}$ and RhoA pathways in cytokinestimulated vascular endothelial cells when EPCR is occupied by protein C. Thromb Haemost 101: 513-520.

40. Bae J, Rezaie A (2008) Protease activated receptor 1 (PAR-1) activation by thrombin is protective in human pulmonary artery endothelial cells if endothelial protein $C$ receptor is occupied by its natural ligand. Thromb Haemost 100: 101-109.

41. Bae J, Yang L, Manithody C (2007) The ligand occupancy of endothelial protein C receptor switches the PAR-1-dependent signaling specificity of thrombin from a permeability-enhancing to a barrierprotective response in endothelial cells. Blood 110: 3909-3916.

42. Feistritzer C, Schuepbach R, Mosnier L, Bush L, Di Cera E, et al. (2006) Protective signaling by activated protein $\mathrm{C}$ is mechanistically linked to protein $\mathrm{C}$ activation on endothelial cells. J Biol Chem 281: 20077-20084.

43. Preston R, Villegas-Mendez A, Sun Y, Hermida J, Simioni P, et al. (2005) Selective modulation of protein C affinity for EPCR and phospholipids by Gla domain mutation. FEBS J 272: 97-108.

44. Yamaji K, Wang Y, Liu Y, Abeyama K, Hashiguchi T, et al. (2005) Activated protein C, a natural anticoagulant protein, has antioxidant properties and inhibits lipid peroxidation and advanced glycation end products formation. Thromb Res 115: 319-325.

45. Bock F, Shahzad K, Vergnolle N, Isermann B (2014) Activated protein C based therapeutic strategies in chronic diseases. Thromb Haemost 111: 610-617.

46. Raedschelders K, Ansley D, Chen D (2012) The cellular and molecular origin of reactive oxygen species generation during myocardial ischemia and reperfusion. Pharmacol Ther 133: 230-255.

47. Iqbal K, Rauoof M, Mir M, Tramboo N, Malik J, et al. (1987) Lipid peroxidation during acute coronary syndromes and its intensification at the time of myocardial ischemia reperfusion. Am J Cardiol 89: 334-337.

48. Hirose K, Okajima K, Taoka Y, Uchiba M, Tagami H, et al. (2000) Activated protein C reduces the ischemia/reperfusion-induced spinal cord injury in rats by inhibiting neutrophil activation. Ann Surg 232: $272-280$.

49. Wang Y, Sinha R, Mosnier L, Griffin J, Zlokovic B (2013) Neurotoxicity of the anticoagulant-selective E149A-activated protein C variant after focal ischemic stroke in mice. Blood Cells Mol Dis 51: 104108.

50. Guo H, Liu D, Gelbard H, Cheng T, Insalaco R, et al. (2004) Activated protein C prevents neuronal apoptosis via protease activated receptors 1 and 3. Neuron 41: 563-572.

51. Zhong Z, Ilieva H, Hallagan L, Bell R, Singh I, et al. (2009) Activated protein C therapy slows ALS-like disease in mice by transcriptionally inhibiting SOD1 in motor neurons and microglia cells. J Clin Invest 119: 3437-3449.

52. Galley H, El Sakka N, Webster N, Lowes D, Cuthbertson B (2008) Activated protein C inhibits chemotaxis and interleukin-6 release by human neutrophils without affecting other neutrophil functions. Br J Anaesth 100: 815-819.

53. van Lenten B, Wagner A, Navab M, Fogelman A (2001) Oxidized phospholipids induce changes in hepatic paraoxonase and ApoJ but not monocyte chemoattractant protein -1 via interleukin-6. J Biol Chem 276: 1923-1929.

54. Song L, Schindler C (2004) IL-6 and the acute phase response in murine atherosclerosis. Atherosclerosis 177: 43-51.

55. Keidar s, Heinrich R, Kaplan M, Heayek T, Aviram M (2001) Angiotensin II administration to atherosclerotic mice increases macrophage uptake of oxidized LDL: a possible role for interleukin-6. Thromb Vasc Biol 21: 1464-1469. 
56. Huber S, Sakkinen P, Conze D, Hardin N, Tracy R (1999) Interleukin-6 exacerbates early atherosclerosis in mice. Arterioscler Thromb Vasc Biol 19: 2364-2367.

57. Nakashima Y, Plump A, Raines E, Breslow J, Ross R (1994) ApoE-deficient mice develop lesions in all phases of atherosclerosis throughout the arterial tree. Ateroscler Thromb 14: 133-140.

58. Elhage R, Clamens S, Besnard S, Mallat Z, Tedgui A, et al. (2001) Involvement of interleukin-6 in atherosclerosis but not in the prevention of fatty streak formation by 17 beta-estradiol in apolipoprotein E-deficient mice. Atherosclerosis 156: 315-320.

59. Spagnoli L, Bonanno E, Sangiorgi G, Mauriello A (2007) Role of Inflammation in Atherosclerosis. J Nucl Med 48: 1800-1815.

60. Hansson G (2001) Immune Mechanisms in Atherosclerosis. Arterioscler Thromb Vasc Biol 21: 18761890.

61. Petäjä J, Pesonen E, Fernández J, Vento A, Rämö O, et al. (1999) Cardiopulmonary bypass and activation of antithrombotic plasma protein C. J Thorac Cardiovasc Surg 118: 422-429.

62. Boisclair M, Lane D, Philippou H, Sheikh S, Hunt B (1993) Thrombin production, inactivation and expression during open heart surgery measured by assays for activation fragments including a new ELISA for prothrombin fragment F1 + 2. Thromb Haemost 70: 253-258.

63. Maehata Y, Miyagawa S, Sawa Y (2012) Activated protein C has a protective effect against myocardial I/R injury by improvement of endothelial function and activation of AKT1. PLoS One 7: e38738.

64. Lay W, Rayner B, Sabaretnam T, Figtree G, Jackson C, et al. (2012) Activated protein C improves left ventricular remodelling after ischemia-reperfusion injury in rats. Int J Cardiol 159: 246-248.

65. Ding J, Tong X, Yang J, Liu Z, Zhang Y, et al. (2010) Activated protein C protects myocardium via activation of anti-apoptotic pathways of survival in ischemia-reperfused rat heart. J Korean Med Sci 25: 1609-1615.

66. Pirat B, Muderrisoglu H, Unal M, Ozdemir H, Yildirir A, et al. (2006) Recombinant human-activated protein $\mathrm{C}$ inhibits cardiomyocyte apoptosis in a rat model of myocardial ischemia-reperfusion. Coron Artery Dis 18: 61-66.

67. Loubele S, Spek C, Leenders P, Hamulyák K, Matthijsen R, et al. (2009) The anti-coagulants ASIS and APC do not protect against renal I/R injury. Inhibition of ischemic organ damage with anti-coagulant agents. Maastricht: Thesis Maastricht University. pp. 91-111.

68. Borissoff J, Otten J, Heeneman S, Leenders P, van Oerle R, et al. (2013) Genetic and pharmacological modifications of thrombin formation in apolipoprotein e-deficient mice determine atherosclerosis severity and atherothrombosis onset in a neutrophil-dependent manner. PLoS One 8: e55784.

69. Wang Y, Zhang Z, Chow N, Davis T, Griffin J, et al. (2012) An activated protein C analog with reduced anticoagulant activity extends the therapeutic window of tissue plasminogen activator for ischemic stroke in rodents. Stroke 43: 2444-2449.

70. Brückner M, Lasarzik I, Jahn-Eimermacher A, Peetz D, Werner C, et al. (2013) High dose infusion of activated protein $\mathrm{C}$ (rhAPC) fails to improve neuronal damage and cognitive deficit after global cerebral ischemia in rats. Neurosci Lett 551: 28-33.

71. Lin X, Li Y, Mei G, Zou F, He D, et al. (2009) Activated protein C can be used as a prophylactic as well as a therapeutic agent for heat stroke in rodents. Shock 32: 524-529.

72. Chen C, Hou C, Cheng K, Tian R, Chang C, et al. (2006) Activated protein C therapy in a rat heat stroke model. Crit Care Med 34: 1960-1966.

73. Teschendorf P, Albertsmeier M, Vogel P, Padosch S, Spöhr F, et al. (2008) Neurological outcome and inflammation after cardiac arrest--effects of protein $C$ in rats. Resuscitation 79: 316-324.

74. Teschendorf P, Padosch S, Del Valle Y, Fuentes D, Peter C, et al. (2009) Effects of activated protein C on post cardiac arrest microcirculation: an in vivo microscopy study. Resuscitation 80: 940-945. 


\section{Chapter 5}

\section{Proteolysis of extracellular histones by activated protein C}

Karin C.A.A. Wildhagen ${ }^{1}$, Jiangfeng $\mathrm{Du}^{1}$, Roy Schrijver ${ }^{1}$, Chris P.M. Reutelingsperger ${ }^{1}$, Gerry A.F. Nicolaes ${ }^{1}$

1. Department of Biochemistry, Cardiovascular Research Institute Maastricht (CARIM), Maastricht University, Maastricht, The Netherlands 


\section{Abstract}

Histones are cationic nuclear proteins that package and order DNA into structural units called nucleosomes. Extracellular histones are cytotoxic and major mediators of death in murine sepsis. Circulating nucleosomes and histones are elevated in patients with inflammation, sepsis and traumatic injury. Activated protein C (APC), an anticoagulant protein that can cleave histones in vitro and in vivo, reduces histone cytotoxicity.

We investigated in vitro cleavage of histone $\mathrm{H} 3$ by human wildtype (wt)-APC and by the human APC variant 5A-APC (1), which has minimal anticoagulant activity. We determined effects of $\mathrm{Ca}^{2+}$ and phospholipids on $\mathrm{H} 3$ cleavage and cytotoxicity. Furthermore, we identified APC cleavage sites in H3.

H3 cleavage by APC is most efficient in the presence of physiological $\mathrm{Ca}^{2+}$ concentrations and phospholipid vesicles containing both phosphatidylethanolamine and phosphatidylserine. Surprisingly, 5A-APC cleaved H3 significantly better than wtAPC. Both wt-APC and 5A-APC cleaved histone $\mathrm{H} 3$ at R26 and R52, resulting in two $\mathrm{H} 3$ fragments of $12.49 \mathrm{kDa}$ (K27-A135) and $9.66 \mathrm{kDa}$ (R53-A135). Both fragments lack in vitro cytotoxic properties towards endothelial cells.

5A-APC therefore can potentially be used for treatment of patients with acute and chronic pathologic conditions characterized by increased histone levels in circulation. The advantages of this variant over wt-APC are that 5A-APC cleaves histones more efficiently and that higher concentrations can be safely administered without risk for bleeding complications. 


\section{Introduction}

Histones are cationic nuclear proteins that package and order DNA into structural units called nucleosomes. Nucleosomes consist of a core octamer containing two copies each of histones H2A, H2B, H3 and H4 surrounded by a superhelix of 146 bp DNA (2). Both the nucleosome core particle and the DNA are bound by linker protein histone H1 to form a higher-order chromatin structure, called chromatosome, which stabilizes the nucleosome (3).

In murine models it was shown that extracellular histones are major mediators of death in sepsis and that mainly histone $\mathrm{H} 3$ and histone $\mathrm{H} 4$ are cytotoxic (4). Increased concentrations of extracellular histones and nucleosomes were found in circulation of patients with systemic inflammation, sepsis and traumatic injury (5-7) and were shown to correlate with disease severity and mortality (8). Extracellular histones and nucleosomes can originate from apoptotic and necrotic cells (6) and from neutrophil extracellular traps (NETs) that are formed after neutrophil activation $(9,10)$. NETs are composed of granular and nuclear constituents and are produced to trap, disarm and kill bacteria extracellularly (10). It was already known for a long time that histones possess bactericidal activity (11). Extracellular histones not only kill bacteria but also host cells such as endothelial cells and thereby contribute to endothelial dysfunction, organ failure and death during sepsis $(4,12)$. The exact molecular mechanism of histone-mediated cytotoxicity is not clear yet. Histones are able to interact directly with membrane phospholipids and may form channels in both plasma and nuclear membranes, causing loss of membrane barrier function (13-15). The tertiary structure of histones and the degree of positive charge play important roles in this activity, which is enhanced in the presence of anionic phospholipids (13). Another line of evidence shows that histones can activate Toll-like receptor (TLR) 2 and TLR4, leading to cytokine production that results in sterile inflammation, tissue injury and death in mouse models (16).

In critically injured trauma patients, the association between increased histone levels and mortality was abrogated by concomitant elevation of activated protein $\mathrm{C}$ (APC) levels (8). Xu et al. (4) showed that APC can target and cleave histones both in vitro and in vivo and thereby reduce their cytotoxicity. APC is best described as an anticoagulant serine protease that, together with its cofactors protein $\mathrm{S}$ and factor $\mathrm{V}(\mathrm{FV})$, regulates thrombin formation by proteolysis of the active coagulation factors $\mathrm{V}(\mathrm{FVa})$ and VIII (FVIIIa) $(17,18)$. It is known that three loops of a positively charged exosite on the surface of APC are important for FVa and FVIIIa binding and cleavage. These loops are the 191 loop (residues 190-193), the calcium binding loop (residues 225-235) and the autolysis loop (residues 302-317) (19, 20). Mutation of three residues in the 191 loop and two residues in the calcium binding loop (KKK191-193AAA and RR229230AA) yields a mutant (5A-APC), firstly described by Mosnier et al. (1), that was found to possess minimal anticoagulant activity but that retains normal cytoprotective activity. 
In the present study we describe the proteolytic cleavage of histone $\mathrm{H} 3$ by wt-APC and 5A-APC and the effect of such cleavage on histone-mediated cytotoxicity to endothelial cells. We furthermore describe the effect of calcium and phospholipid vesicles on the efficiency of histone $\mathrm{H} 3$ cleavage by APC and identify the cleavage sites of APC in histone $\mathrm{H} 3$.

\section{Materials \& Methods}

\section{Expression and purification of recombinant human PC}

Recombinant human 5A-PC (containing alanine substitutions of residues K191, K192, K193, R229 and R230) was obtained by PCR-based site-directed mutagenesis of wt-PC cDNA. The cDNA of wt-PC and 5A-PC were inserted into the eukaryotic expression vector pRc/CMV (Invitrogen), transfected into HEK293 cells (CRL-1573 ATCC) and recombinant 5A-PC was purified and characterized as described earlier $(21,22)$.

The purity and integrity of wt-PC and 5A-PC preparations was evaluated by SDSPAGE. Wt-PC and 5A-PC concentrations were quantified by measurement of absorbance at $280 \mathrm{~nm}$ using an absorption coefficient of $14.5(280 \mathrm{~nm}, 1 \%, 1 \mathrm{~cm})$ and by ELISA, employing the horse anti-human PC polyclonal antibody PAHPC-H (HTI, VT, USA) as a catcher antibody and the horseradish peroxidase-conjugated anti-human PC polyclonal (Dako P0374, Glostrup, Denmark) as a detecting antibody (23).

\section{Activation of $P C$ and catalytic activity against small substrates}

Wt-PC and 5A-PC were activated with a $1 \mathrm{ml}$ HiTrap NHS-Activated HP column (GE Health care, Little Chalfont, United Kingdom) to which 50 Units of carrier-free Protac (Pentapharm, Basel, Switzerland) were conjugated. Up to $0.5 \mathrm{mg}$ of (5A) PC was incubated for 2 hours on the column at RT, and was next eluted and concentrated with 10K Macrosep Advance Centrifugal Devices (Pall Corporation, Port Washington, $\mathrm{NY)}$. Complete activation was ascertained by measuring the amidolytic activity of APC with S-2366 (Chromogenix, Milan, Italy) directly and after 60 minutes incubation in the presence of additionally added ( 0.05 units $/ \mathrm{ml}$ final concentration) Protac. For both wt-PC and 5A-PC, completion of activation was verified by SDS PAGE analysis. APC concentrations were determined from the absorbance at $280 \mathrm{~nm}$ and by the abovedescribed PC ELISA.

\section{Preparation of phospholipid vesicles}

Small unilamellar phospholipid vesicles were prepared as described (24). Synthetic lipids 1,2 dioleoyl-sn-glycero-3-phosphoserine (DOPS), 1,2 dioleoyl-sn-glycero-3- 
phosphocholine (DOPC) and 1,2 dioleoyl-sn-glycero-3-phosphoethanolamine (DOPE) were obtained from Avanti Polar Lipids (Alabaster, Alabama).

\section{Cell culture}

The human endothelial cell line EA.hy926, kindly provided by Dr. Dariusz Leszczynski (STUK - Radiation and Nuclear Safety Authority, Helsinki, Finland), was maintained in Dulbecco's modified eagle's medium (Lonza, Basel, Switzerland) supplemented with penicillin, streptomycin, L-glutamine, 10\% fetal bovine serum and HAT supplement (all Life Technologies, Carlsbad, CA) in a humidified atmosphere at $37^{\circ} \mathrm{C}$ and $5 \% \mathrm{CO}_{2}$.

\section{Histone $\mathrm{H} 3$ cleavage by $A P C$}

$40 \mu \mathrm{g} / \mathrm{ml}$ Purified calf thymus histone H3 (Roche, Basel, Switzerland) was incubated for 30 minutes at $37^{\circ} \mathrm{C}$ in buffer ( $25 \mathrm{mM}$ Hepes, $150 \mathrm{mM} \mathrm{NaCl}$, pH: 7.7) containing 0-1000 nM wt-APC, 0-500 nM 5A-APC, 0-5 $\mathrm{mM} \mathrm{CaCl}_{2}$ and 0-200 $\mu \mathrm{M}$ phospholipid vesicles (DOPC, 20:80 DOPS:DOPC (mol:mol) or 20:60:20 DOPS:DOPC:DOPE (mol:mol:mol)). Next, cell cytotoxicity and histone H3 cleavage were analyzed with respectively flow cytometry and SDS-PAGE followed by Western blotting (see method histone $\mathrm{H} 3$ determination).

\section{Flow cytometry}

EA.hy 926 cells were cultured in 24 well plates $\left(1 \times 10^{5}\right.$ cells/well $)$ to produce confluent monolayers, washed with PBS and incubated for exactly 30 minutes with (cleaved) histone $\mathrm{H} 3$ (prepared as described before) at $37^{\circ} \mathrm{C}$. Afterwards buffer containing detached dead cells was collected in a fresh tube and the remaining cells were detached with $0.05 \%$ trypsin/EDTA during exactly 5 minutes and transferred to the same tube. Cells were pelleted, diluted to $1 \times 10^{6}$ cells $/ \mathrm{ml}$ and incubated for $10 \mathrm{~min}$ on ice in buffer $(10 \mathrm{mM}$ Hepes, $150 \mathrm{mM} \mathrm{NaCl}, 5 \mathrm{mM} \mathrm{KCl}, 1 \mathrm{mM} \mathrm{MgCl}, 2.5 \mathrm{mM} \mathrm{CaCl}_{2}, \mathrm{pH}$ : 7.4) containing $2.5 \mu \mathrm{g} / \mathrm{ml}$ propidium iodide (PI) (Life Technologies, Carlsbad, CA). Percentages of PI positive cells and mean fluorescence of PI were determined with a flow cytometer (BD Accuri ${ }^{\mathrm{TM}}$ C6, Franklin Lakes, New Jersey) and BD CFlow plus software.

\section{Western blotting}

Ten times diluted samples of (cleaved) histone H3 (prepared as described above) were analysed via SDS-PAGE gel electrophoresis (6-15\%) and transferred to PVDF membranes (Pall Corporation, Port Washington, NY) by a semi-dry blotting technique. After blocking, the membranes were overnight incubated at $4{ }^{\circ} \mathrm{C}$ with a rabbit polyclonal histone H3 antibody mapping an epitope at the C-terminus (1:1000, sc-8654-R, Santa 
Cruz Biotechnology, Heidelberg, Germany), followed by incubation with a biotinylated goat anti-rabbit IgG antibody (1:1000, Vector Laboratories, Burlingame, CA) for 30 minutes at RT and finally with StreptABComplex/AP solution (1:3000, Dako, Glostrup, Denmark) for 30 minutes at RT. Protein bands were detected by BCIP/NBT (SigmaAldrich, St. Louis, Missouri) and density of histone H3 bands was quantified by ImageJ software (x86, Wayne Rasband) and percentages of the total intensity of all bands per lane were calculated.

\section{Prediction of cleavage sites and histone $\mathrm{H} 3$ fragments}

The bovine histone H3.1 sequence was retrieved from the uniprot database (ID: P68432). The sequence contained 135 amino acids (after the initiator methionine being removed). The molecular weight (MW) of fragments after APC-catalyzed cleavage was estimated from Western blot analysis. We defined an algorhythm to predict the sequence of detectable histone $\mathrm{H} 3$ fragments based on the following assumptions: 1) APC is able to cleave C-terminal of lysine $(\mathrm{K})$ or arginine $(\mathrm{R})$ when consecutive $\mathrm{P} 1$ to P1' amino acids are: KK, KS, RE, RG, RH, RK, RM, RN or RR (25-27); 2) the fragments must contain the known epitope of the histone $\mathrm{H} 3$ antibody used for Western blotting (at least 10 amino acids from D106 to A135); 3) there are no post-translational modifications in the histone structure. Potential cleavage sites in the bovine histone H3.1 were identified. The molecular weights of potential histone $\mathrm{H} 3$ fragments were calculated and compared to the estimated MW of fragments obtained via Western blot analysis.

\section{$N$ terminal sequencing by Edman degradation}

A sample containing different histone $\mathrm{H} 3$ cleavage products was transferred from a SDS-PAGE gel to a PVDF membrane, stained with Coomassie Blue and two bands were cut out for $\mathrm{N}$ terminal sequencing analysis. The analysis was performed by Alphalyse (Odense, Denmark) on an ABI Procise 494 sequencer. The cyclic procedure was performed in 3 steps. In step 1 PITC reagent was coupled to the N-terminal amino group under alkaline conditions. In step 2 the $\mathrm{N}$-terminal residue was cleaved in acidic media. In step 3, the PITC coupled residue was transferred to a flask, converted to a PTH-residue and identified by HPLC chromatography. The next cycle was then started for identification of the next $\mathrm{N}$-terminal residue. If the sample contained more than one protein or $\mathrm{N}$-terminal variants of the same protein then residues will be shown as $\mathrm{X} / \mathrm{Y} / \mathrm{Z}$. Due to differences in the chemical reaction for certain amino acids the identification of some residues may be uncertain and was shown in parentheses $(\mathrm{X} / \mathrm{Y})$. 


\section{Statistical analyses}

Analysis of data was performed in GraphPad Prism 5. Results in Figure 2 are expressed as mean \pm SEM and independent samples $T$ tests were used to analyze differences between groups. For analysis of correlation in Figure 3 Coefficients of determination $\left(\mathrm{R}^{2}\right)$, Pearson correlation coefficients ( $\mathrm{r}$ ) and two tailed P-values were calculated. In all cases $\mathrm{P}$-values $<0.05$ were regarded as statistically significant.

\section{Results}

Histone $H 3$ cleavage is more efficient by $5 A-A P C$ than by wt-APC and influenced by calcium

Histone H3 cleavage by wt-APC was followed over time by Western blotting (Figure 1A). Uncleaved, full length histone appears as a $\sim 15 \mathrm{kDa}$ band $(\mathrm{t}=0)$, corresponding to its calculated weight of $15.25 \mathrm{kDa}$. Within 1 minute of incubation, $94 \%$ of full-length histone $\mathrm{H} 3$ was cleaved and within 3 minutes all full-length histone $\mathrm{H} 3$ disappeared and different cleavage products appeared. Finally, after 1 hour of incubation two histone H3 cleavage products with estimated MW of $12.32 \mathrm{kDa}$ and $9.70 \mathrm{kDa}$ remained and can be considered as end products.

Cleavage of histone $\mathrm{H} 3$ by wt-APC was compared with cleavage by the 5A-APC mutant, which has minimal anticoagulant activity but normal cytoprotective capacity. Figure 1B shows that 5A-APC cleaves histone H3 more efficiently compared to wt-APC. At concentrations of 50 and $200 \mathrm{nM}$, after a fixed time interval of 30 minutes incubation, wt-APC has cleaved approximately $50 \%$ of full-length histone $\mathrm{H} 3$, while $5 \mathrm{~A}-\mathrm{APC}$ has cleaved all full-length histone $\mathrm{H} 3$. When higher concentrations $(500 \mathrm{nM})$ of wt-APC are used, histone $\mathrm{H} 3$ was cleaved completely within 30 minutes of incubation and the Western blot of final reaction products was comparable to that of 5A-APC.

Next we investigated the effect of calcium on histone H3 cleavage by APC. Calciumions are important for procoagulant and anticoagulant processes in general and for the maintenance of the $\mathrm{Ca}^{2+}$-dependent conformation of APC, for phospholipid binding of APC and hence for APCs anticoagulant activity in particular (28). We found that $\mathrm{CaCl}_{2}$ concentrations of $1 \mathrm{mM}$ and $2.5 \mathrm{mM}$, which are in a range of what is considered as "physiological", are optimal for histone H3 cleavage by wt-APC (Figure 1C) and $5 \mathrm{~A}-\mathrm{APC}$ (not shown). In either the absence of $\mathrm{CaCl}_{2}$ or in the presence of higher $\mathrm{CaCl}_{2}$ concentrations $(\geq 5 \mathrm{mM})$ APC cleaved histone $\mathrm{H} 3$ less efficient. 


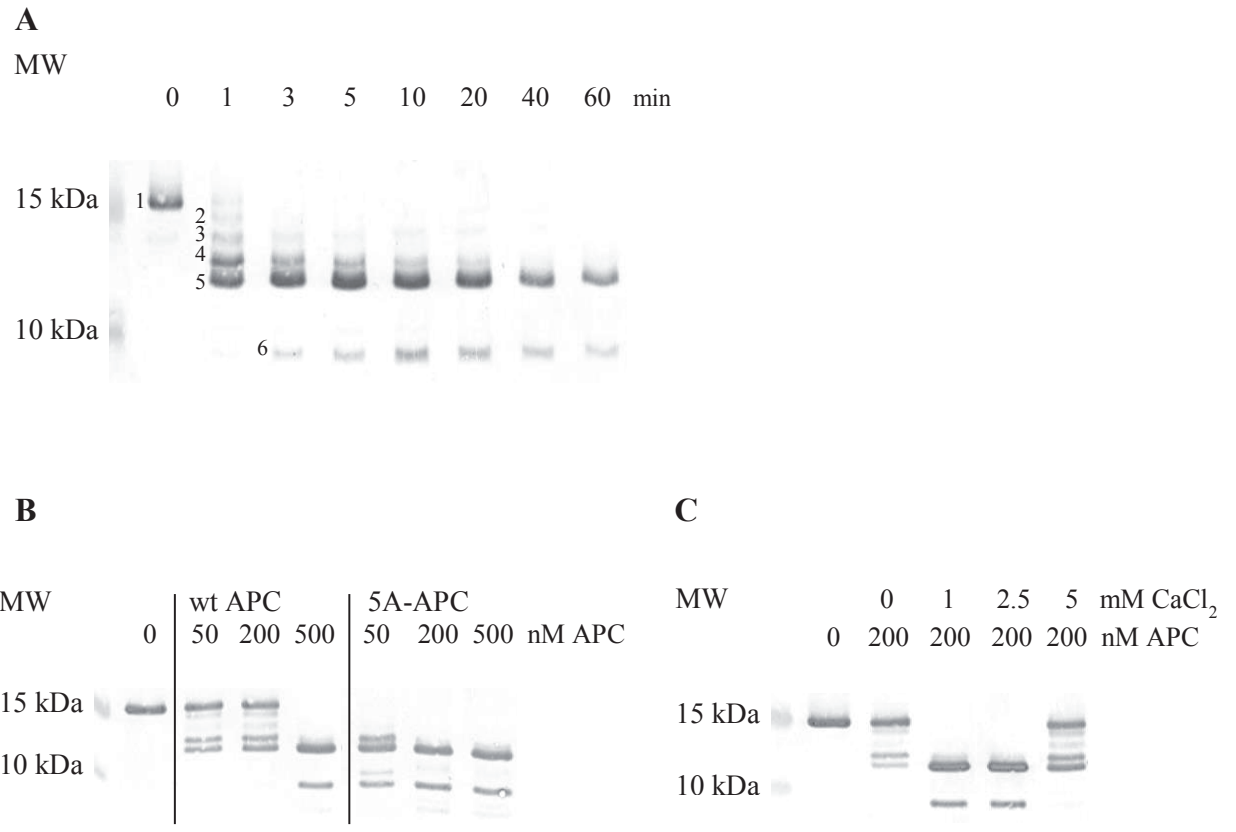

\section{Figure 1. Histone $\mathrm{H} 3$ cleavage by wt and 5A-APC}

A) $5 \mu \mathrm{g} / \mathrm{ml}$ histone $\mathrm{H} 3$ was incubated for $0-60 \mathrm{~min}$ at $37^{\circ} \mathrm{C}$ with $85 \mathrm{nM}$ wt APC and $5 \mathrm{mM} \mathrm{CaCl} 2$. The estimated MW of bands: 1) $15.25 \mathrm{kD}$, 2) $14.55 \mathrm{kDa}$, 3) $13.77 \mathrm{kDa}$, 4) $13.00 \mathrm{kDa}$, 5) $12.32 \mathrm{kDa}$ and 6) 9.70 $\mathrm{kDa}$ B) $40 \mu \mathrm{g} / \mathrm{ml}$ histone $\mathrm{H} 3$ was incubated for $30 \mathrm{~min}$ at $37^{\circ} \mathrm{C}$ with $50-500 \mathrm{nM}$ wt APC or $50-500 \mathrm{nM}$ 5A-APC. C) $40 \mu \mathrm{g} / \mathrm{ml}$ histone $\mathrm{H} 3$ was incubated for $30 \mathrm{~min}$ at $37^{\circ} \mathrm{C}$ with $200 \mathrm{nM}$ wt APC and $0-5 \mathrm{mM}$ $\mathrm{CaCl}_{2}$. All samples were analyzed with SDS-PAGE followed by Western blot using a rabbit polyclonal histone $\mathrm{H} 3$ antibody mapping an epitope at the $\mathrm{C}$-terminus. Western blots are representative images of at least 3 experiments.

\section{Cleavage of histone $\mathrm{H} 3$ decreases its cytotoxicity to endothelial cells}

To investigate the effect of histone $\mathrm{H} 3$ cleavage on its cytotoxicity, histone $\mathrm{H} 3$ cleavage products were incubated for 30 minutes with endothelial cells, followed by determination of cell viability by flow cytometry. In the absence of histone H3, APC did not influence cell viability of endothelial cells in any of the experimental conditions used (not shown). Figure $2 \mathrm{~A}$ shows that $30 \mathrm{~min}$ pre-incubation of histone $\mathrm{H} 3$ with different concentrations of either wt-APC or 5A-APC dose-dependently decreased cytotoxicity of histone $\mathrm{H} 3$ and resulted in increased remaining cell viability of endothelial cells. In agreement with the increased cleavage of histone $\mathrm{H} 3$ by $5 \mathrm{~A}-\mathrm{APC}$ observed by Western blotting, we observed that 5A-APC destroys $\mathrm{H} 3$ cytotoxicity more efficiently than wtAPC (Figure 2A).

Figure $2 \mathrm{~B}$ shows that pre-incubation of histone $\mathrm{H} 3$ with $\mathrm{APC}$ in the presence of $1 \mathrm{mM}$ and $2.5 \mathrm{mM} \mathrm{CaCl}_{2}$ reduces histone-mediated cytotoxicity. The percentage of viable cells increased significantly from $44.73 \%$ in the absence of calcium to $73.74 \%$ in the 
presence of $1 \mathrm{mM} \mathrm{CaCl}_{2}(\mathrm{P}=0.0270)$ and $68.15 \%$ in the presence of $2.5 \mathrm{mM} \mathrm{CaCl}_{2}$ $(\mathrm{P}=0.0199)$. This is in agreement with the increased cleavage of histone $\mathrm{H} 3$ observed in the presence of these $\mathrm{CaCl}_{2}$ concentrations. In the absence of APC addition of $\mathrm{CaCl}_{2}$ does not have any effect on cell viability.
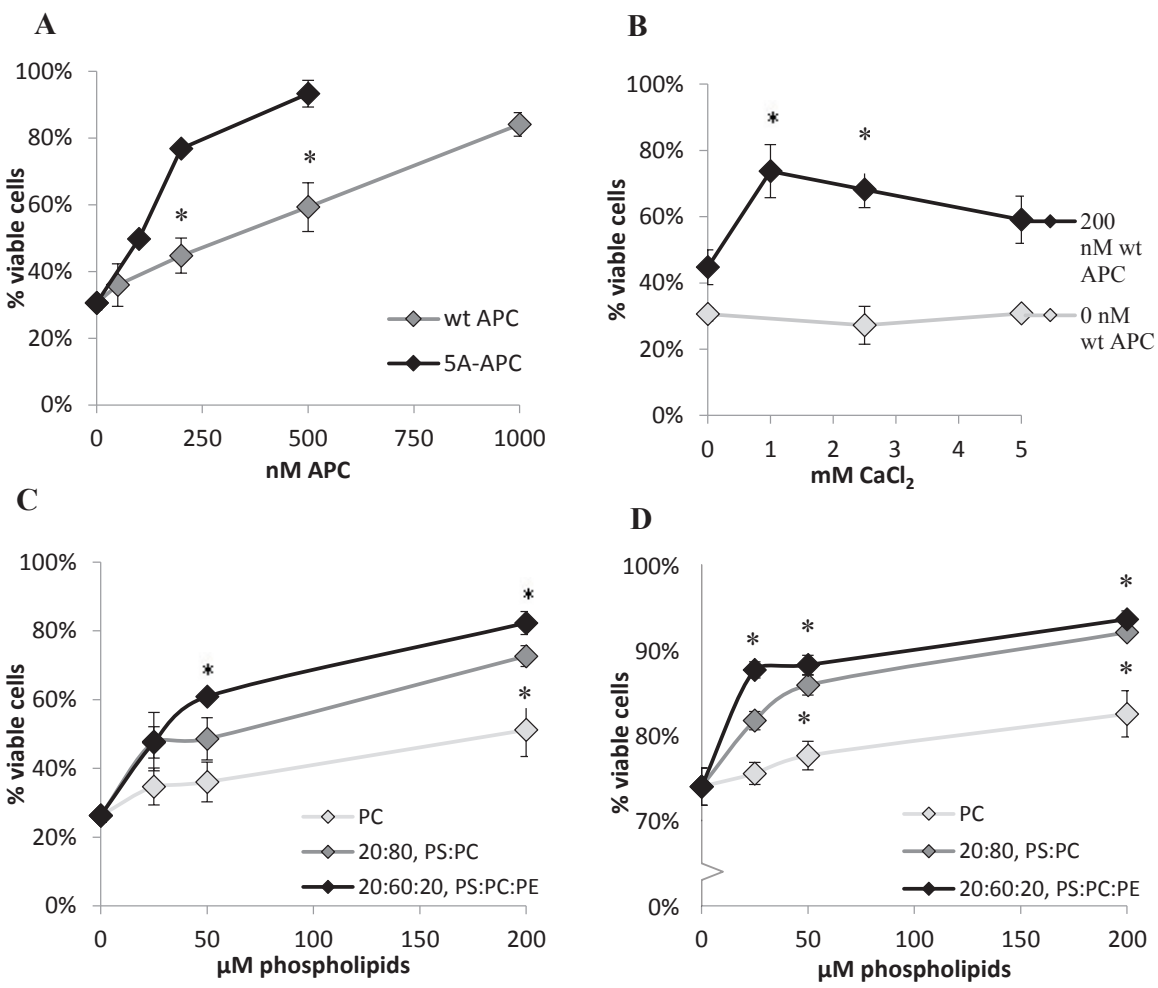

Figure 2. Effect of $\mathrm{APC}, \mathrm{CaCl}_{2}$ and phospholipids on histone $\mathrm{H} 3$ cytotoxicity to endothelial cells In all conditions $40 \mu \mathrm{g} / \mathrm{ml}$ histone $\mathrm{H} 3$ was pre-incubated for $30 \mathrm{~min}$ at $37^{\circ} \mathrm{C}$ with different concentrations of APC, $\mathrm{CaCl}_{2}$ and phospholipids. Samples were afterwards incubated for $30 \mathrm{~min}$ with EA.hy926 cells, followed by flow cytometric analysis of cell viability with propidium iodide (PI). A) Pre-incubation of histone H3 with $0-1000 \mathrm{nM}$ wt APC or $0-500 \mathrm{nM} 5 \mathrm{~A}-\mathrm{APC} . * \mathrm{P}<0.05$ for comparison of wt APC and 5A-APC. $\mathrm{n}=2-9$ per condition. B) Pre-incubation of histone H3 with 0 or $200 \mathrm{nM}$ wt APC and $0-5 \mathrm{mM}$ $\mathrm{CaCl}_{2} * \mathrm{P}<0.05$ compared to $0 \mathrm{mM} \mathrm{CaCl}_{2} . \mathrm{n}=2-7$ per condition. C) Pre-incubation of histone $\mathrm{H} 3$ with 2.5 $\mathrm{mM} \mathrm{CaCl}_{2}$ and 0-200 $\mu \mathrm{M}$ phospholipids PC, 20:80 PS:PC or 20:60:20 PS:PC:PE in the absence of APC. $* \mathrm{P}<0.05$ compared to $0 \mu \mathrm{M}$ phospholipids. $\mathrm{n}=3$ per condition. D) Pre-incubation of histone H3 with 2.5 $\mathrm{mM} \mathrm{CaCl} 2$ and 0-200 $\mu \mathrm{M}$ phospholipids PC, 20:80 PS:PC or 20:60:20 PS:PC:PE in the presence of $200 \mathrm{nM}$ wt APC. $* \mathrm{P}<0.05$ compared to $0 \mu \mathrm{M}$ phospholipids. $\mathrm{n}=3$ per condition. 


\section{Effect of phospholipid vesicle composition on histone $\mathrm{H} 3$ cleavage by APC}

It is known that histones interact with membrane phospholipids $(13,29)$ and Xu et al. (4) have shown that APC cleaves histone H3 and histone H4 better in the presence of liposomes containing phosphatidylethanolamine (PE). We here investigated the effect of phospholipid vesicle composition on the cytotoxicity of histone $\mathrm{H} 3$ towards endothelial cells in the presence and absence of APC. Histone H3 was pre-incubated with different concentrations of phospholipid vesicles containing only PC, 20:80 (mol:mol) phosphatidylserine (PS): phospatidylcholine (PC) or 20:60:20 (mol:mol:mol) PS:PC:PE in the presence or absence of $200 \mathrm{nM}$ wt-APC. In the absence of APC, none of the phospholipid vesicle compositions induced any histone $\mathrm{H} 3$ cleavage (not shown, as judged by Western blot analysis). Nevertheless, all phospholipid compositions dosedependently protected endothelial cells against histone $\mathrm{H} 3$ cytotoxicity. Figure 2C shows that PC has only a minor, non-significant, effect on H3 cytotoxicity, 20:80 PS:PC has an intermediate effect that was significant at a concentration of $200 \mu \mathrm{M}(\mathrm{P}=0.003)$, and 20:60:20 PS:PC:PE phospholipids were most effective in reducing H3 cytotoxicity with effects reaching significance at both $50 \mu \mathrm{M}(\mathrm{P}=0.0012)$ and $200 \mu \mathrm{M}(\mathrm{P}=0.0022)$. Thus, the presence of phospholipid vesicles, even in the absence of APC, can protect endothelial cells against the cytotoxic effects of histone $\mathrm{H} 3$.

The residual cell viability in the absence of added lipid vesicles increased from $\sim 25 \%$ to $\sim 75 \%$ by addition of $200 \mathrm{nM}$ wt-APC. Addition of 20:80 PS:PC or 20:60:20 PS:PC:PE phospholipid vesicles resulted in increased histone H3 cleavage by APC (not shown) and an additional stimulation of cell protection (Figure 2D). Phospholipids that contain only PC do not significantly enhance the effect of APC on cell viability (at $200 \mu \mathrm{M}$ PC, $\mathrm{P}=0.1156$ ), while 20:80 PS:PC phospholipids enhance the effect of APC at 50 $\mu \mathrm{M}(\mathrm{P}=0.0171)$ and $200 \mu \mathrm{M}(\mathrm{P}=0.0027)$. Phospholipid vesicles that contain both PS and $\mathrm{PE}$ significantly increase cell viability at concentrations of $25 \mu \mathrm{M}(\mathrm{P}=0.0093)$, $50 \mu \mathrm{M}(\mathrm{P}=0.0032)$ and $200 \mu \mathrm{M}(\mathrm{P}=0.0025)$. So, both in the absence and presence of APC 20:60:20 PS:PC:PE phospholipid vesicles protect endothelial cells best against the cytotoxic effects of histone $\mathrm{H} 3$, while overall protection in the presence of APC exceeds that seen in its absence.

\section{Histone $\mathrm{H} 3$ cleavage products with estimated $M W$ of $12.32 \mathrm{kDa}$ and $9.70 \mathrm{kDa}$ are not cytotoxic to endothelial cells}

The effect of histone $\mathrm{H} 3$ cleavage on its cytotoxicity was further analysed as is represented in Figure 3. We performed semi-quantitative analysis of Western blots by comparison of band intensities of full-length histone $\mathrm{H} 3$ (Figure $3 \mathrm{~A}$ ) and the cleavage end products of $12.32 \mathrm{kDa}$ (Figure 3B) and $9.70 \mathrm{kDa}$ (Figure 3C), for all available different conditions tested in different experiments that included wt-APC in either the presence or absence of $\mathrm{CaCl}_{2}$. Next, we correlated the Western blot band intensities to 
cell viability of endothelial cells in culture determined by flow cytometry 30 minutes after addition of fragments. In flow cytometry measurements in the absence of histone H3, APC did not influence cell viability (not shown). Figure $3 \mathrm{~A}$ shows that there is a significant negative correlation between the amount of full-length histone $\mathrm{H} 3$ present and cell viability of endothelial cells ( $\mathrm{P}>0.0001$, Pearson $\mathrm{r}=-0.8076, \mathrm{R}^{2}=0.6523$ ). The decrease of the full-length histone $\mathrm{H} 3$ band, as is observed during APC-catalyzed proteolysis of $\mathrm{H} 3$, is accompanied by an increase in both the $12.32 \mathrm{kDa}$ and $9.70 \mathrm{kDa}$ histone $\mathrm{H} 3$ bands. The band intensity of both of these fragments on Western blot showed a significant positive correlation with cell viability of endothelial cells $(12.32 \mathrm{kDa}$ fragment: $\mathrm{P}>0.0001$, Pearson $\mathrm{r}=0.8435, \mathrm{R}^{2}=0.7114 ; 9.70 \mathrm{kDa}$ fragment: $\mathrm{P}>0.0001$, Pearson $\left.r=0.8235, R^{2}=0.6782\right)$. From this we conclude that both histone $\mathrm{H} 3$ cleavage products do not possess cytotoxicity towards endothelial cells.
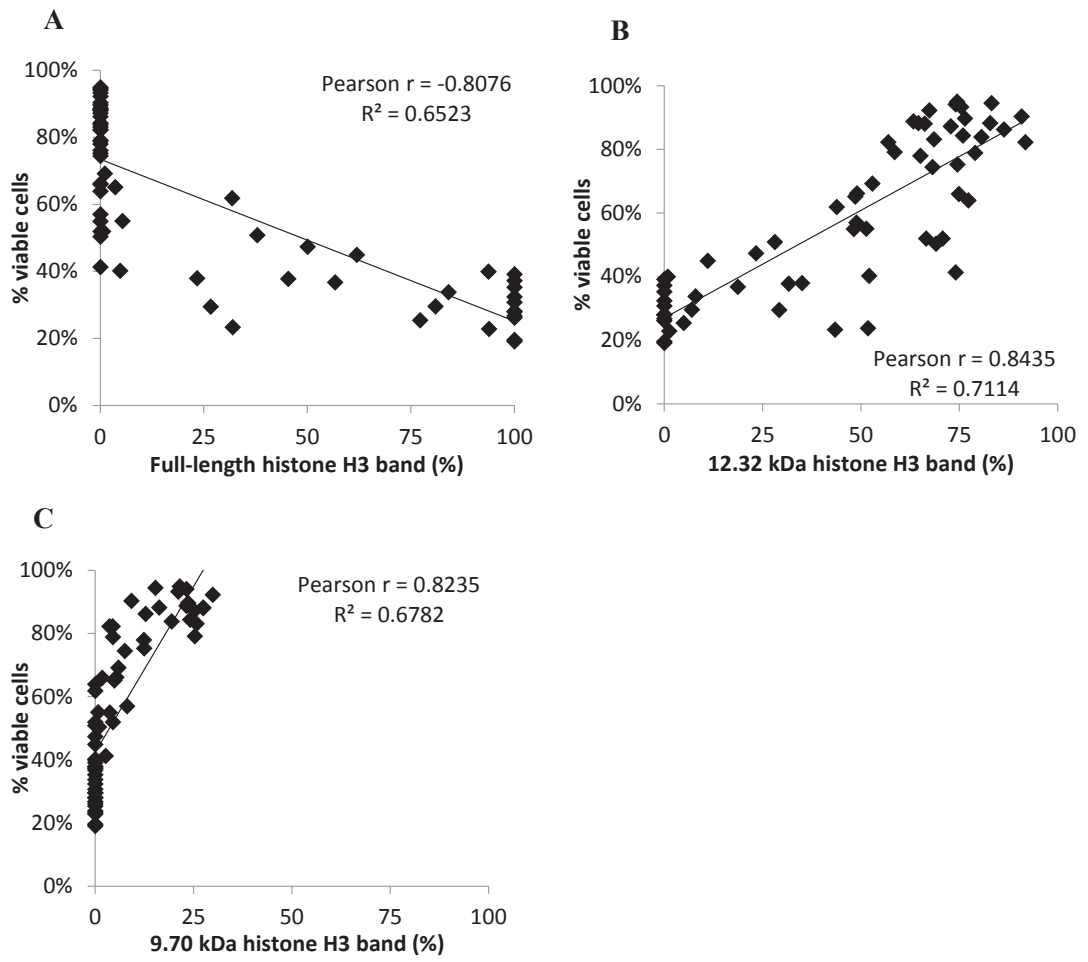

\section{Figure 3. Cleavage of histone $\mathrm{H} 3$ correlates with its cytotoxicity to endothelial cells}

$40 \mu \mathrm{g} / \mathrm{ml}$ histone $\mathrm{H} 3$ was incubated for $30 \mathrm{~min}$ at $37{ }^{\circ} \mathrm{C}$ with different concentrations of wt APC and $\mathrm{CaCl}_{2}$. Incubated $\mathrm{H} 3$ samples were analyzed i) on cleaved products using SDS-PAGE and Western blot analysis and ii) on cytotoxicity by 30 min incubation with EA.hy926 cells followed by flow cytometric analysis of cell viability. ImageJ software was used to quantify the density of three different histone $\mathrm{H} 3$ bands (see arrows figure 1A): full-length histone $\mathrm{H} 3$ band (A), $12.32 \mathrm{kDa}$ histone $\mathrm{H} 3$ band (B) and 9.70 $\mathrm{kDa}$ histone $\mathrm{H} 3$ band (C). Coefficients of determination (R2) and Pearson correlation coefficients ( $\mathrm{r}$ ) were calculated in GraphPad Prism 5. 


\section{Prediction of $A P C$ cleavage sites in bistone $\mathrm{H} 3$}

Based on our assumptions, the developed algorhythm identified 13 potential cleavage sites in histone H3 (R8, K9, R17, R26, K27, K36, R49, R52, K56, R63, R72, R128 and R131) and 38 potentially detectable histone $\mathrm{H} 3$ fragments. The MW of these fragments (Table 1) was compared with the estimated MW of fragments that were obtained after Western blot analysis (Figure 1: 14.55, 13.77, 13.00, 12.32 and $9.70 \mathrm{kD}$ ). The fragments that matched the predicted MW of the fragments best were marked in dark grey and other possible fragments that had a maximum MW deviation of $0.2 \mathrm{kDa}$ were marked in light grey in table 1.

Table 1: Molecular weights of predicted histone $\mathrm{H} 3$ fragments based on our algorhythm.

\begin{tabular}{|l|c|l|l|l|c|}
\hline Fragment & $\begin{array}{c}\text { Molecular } \\
\text { Weight (kDa) }\end{array}$ & Fragment & $\begin{array}{c}\text { Molecular } \\
\text { Weight (kDa) }\end{array}$ & Fragment & $\begin{array}{c}\text { Molecular } \\
\text { Weight (kDa) }\end{array}$ \\
\hline G132-A135 & 0.41 & E50-R128 & 9.22 & K27-A135 & $\mathbf{1 2 . 4 9}$ \\
\hline R129-R131 & 0.43 & R53-R131 & 9.25 & K18-R128 & 12.62 \\
\hline R129-A135 & 0.84 & E50-R131 & $\mathbf{9 . 6 4}$ & K18-R131 & $\mathbf{1 3 . 0 4}$ \\
\hline E73-R128 & 6.29 & R53-A135 & $\mathbf{9 . 6 6}$ & S10-R128 & 13.37 \\
\hline E73-R131 & 6.72 & E50-A135 & 10.06 & K18-A135 & 13.46 \\
\hline E73-A135 & 7.13 & K37-R128 & 10.75 & K9-R128 & 13.50 \\
\hline K64-R128 & 7.43 & K37-R131 & 11.18 & S10-R131 & $\mathbf{1 3 . 8 0}$ \\
\hline K64-R131 & 7.86 & S28-R128 & 11,52 & K9-R131 & $\mathbf{1 3 . 9 3}$ \\
\hline S57-R128 & 8.25 & K37-A135 & 11.59 & S10-A135 & 14.21 \\
\hline K64-A135 & 8.27 & K27-R128 & 11.65 & K9-A135 & 14.34 \\
\hline S57-R131 & 8.67 & S28-R131 & 11.95 & A1-R128 & 14.41 \\
\hline R53-R128 & 8.82 & K27-R131 & 12.07 & A1-R131 & 14.84 \\
\hline S57-A135 & 9.08 & S28-A135 & 12.36 & A1-A135 & 15.25 \\
\hline
\end{tabular}

The bovine histone H3.1 sequence was retrieved from the uniprot database (ID: P68432). Based on the assumptions described in the methods section, potential cleavage sites in the histone $\mathrm{H} 3$ sequence were identified and the molecular weight of possible fragments after APC cleavage was calculated. Uncleaved, full length histone $\mathrm{H} 3$ (A1-A135) has a molecular weight (MW) of $15.25 \mathrm{kDa}$. Based on semi-quantitative Western blot analysis we estimated the MW of APC cleavage products $2 \mathrm{t} / \mathrm{m} 6$ (see figure 1 ) to be 14.55 , $13.77,13.00,12.32$ and $9.70 \mathrm{kD}$. The fragments that matched our predicted MW best are marked in dark grey and other possible fragments that deviated maximum $0.2 \mathrm{kDa}$ from our predicted MW were marked in light grey.

To further verify the identity of APC-cleavage sites we performed N-terminal sequencing to determine the first five $\mathrm{N}$-terminal amino-acids of the two end products of histone H3 cleavage (indicated as bands 5 and 6 in Figure 1). In both fragments 3 consecutive amino-acids could be determined with 100\% certainty (Figure 4). Both of these amino- 
acid sequences were only present at one position in the histone $\mathrm{H} 3$ sequence, so the cleavage sites could be exactly determined.

The location of these cleavage sites is at the R26-K27 and at the R52-R53 peptide bond. The resulting histone $\mathrm{H} 3$ fragments were based on the bovine histone $\mathrm{H} 3.1$ sequence retrieved from the uniprot database (ID: P68432) determined at $12.49 \mathrm{kDa}$ (K27-A135, indicated as band 5, Figure 1) and $9.66 \mathrm{kDa}$ (R53-A135, indicated as band 6) and were correctly identified by our algorhythm. Other predicted cleavage sites based on our algorhythm are R8/K9, R17, R128 and R131, resulting in the histone H3 fragments K18-R131 (13.04 kDa, indicated as band 4), S10-R131/K9-R131 (13.80/13.93 kDa, indicated as band 3) and A1-R128 (14.84 kDa, indicated as band 2).

A

Sequence bovine histone $\mathrm{H3}$ :

\begin{tabular}{|c|c|}
\hline Residue & Amino Acid \\
\hline 1 & $\mathrm{~K} /(\mathrm{G} / \mathrm{A} / \mathrm{R})$ \\
\hline 2 & $\mathrm{~S} /(\mathrm{P})$ \\
\hline 3 & $\mathrm{~A}$ \\
\hline 4 & $\mathrm{P}$ \\
\hline 5 & $\mathrm{~A}$ \\
\hline
\end{tabular}

ARTKQTARKS TGGKAPRKQL ATKAARKSAP ATGGVKKPHR YRPGTVALRE IRRYQKSTEL LIRKLPFQRL VREIAQDFKT DLRFQSSAVM ALQEACEAYL VGLFEDTNLC AIHAKRVTIM PKDIQLARRI RGERA

MW

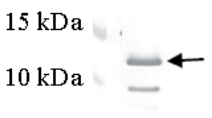

B

\begin{tabular}{|c|c|}
\hline Residue & Amino Acid \\
\hline 1 & $(\mathrm{~T} / \mathrm{G} / \mathrm{R} / \mathrm{K})$ \\
\hline 2 & $\mathrm{Y}$ \\
\hline 3 & $\mathrm{Q}$ \\
\hline 4 & $\mathrm{~K}$ \\
\hline 5 & $\mathrm{~S} / \mathrm{A})$ \\
\hline
\end{tabular}

Sequence bovine histone H3:

ARTKQTARKS TGGKAPRKQL ATKAARKSAP ATGGVKKPHR YRPGTVALRE IRRYQKSTEL LIRKLPFQRL VREIAQDFKT DLRFQSSAVM ALQEACEAYL VGLFEDTNLC AIHAKRVTIM PKDIQLARRI RGERA

\section{Figure 4: APC cleaves histone $\mathrm{H} 3$ at arginine (R)26 and R52}

Edman $\mathrm{N}$ terminal sequencing was used to determine the first five $\mathrm{N}$ terminal amino acids of the histone H3 fragments with estimated MW of $12.32 \mathrm{kDa}(\mathbf{A})$ and $9.70 \mathrm{kDa}(\mathbf{B})$. Sequence of bovine Histone H3.1 (Uniprot P68432) is shown with the first five $\mathrm{N}$ terminal amino acids of the fragments in bold and underlined. The sequences of both histone $\mathrm{H} 3$ fragments are highlighted in grey. 


\section{Discussion}

We have shown that APC-catalysed cleavage of histone $\mathrm{H} 3$ is accelerated in the presence of $\mathrm{CaCl}_{2}$ concentrations with an apparent optimal $\mathrm{CaCl}_{2}$ concentration at the near physiological concentration range of 1 to $2.5 \mathrm{mM}$. This optimum in proteolysis is reminiscent of that found for the cleavage of FVa by APC (30) which also shows an optimum at $\sim 2 \mathrm{mM}$. Calcium ions are in general important for pro- and anticoagulant processes and in case of APC for the maintenance of the overall structure and its phospholipid binding capacities (28).

We observed that phospholipids not only accelerate cleavage of histone H3 by APC, but surprisingly also reduced the cytotoxicity of histone $\mathrm{H} 3$ in the absence of APC. The latter activity may be explained by sequestration of histone $\mathrm{H} 3$ by phospholipid vesicles since it has been shown that histones can interact directly with membrane phospholipids and are able to transverse lipid bilayers of large unilamellar vesicles and multilamellar vesicles $(13,15)$.

In this study we used small unilamellar vesicles that are apparently able to interact with histones and thereby prevent the cytotoxic effects of histones, as evidenced from increased endothelial cell survival. The best protective effect was reached when the phospholipid vesicles did not only contain the neutral phospholipid PC, but also the net negative phospholipid PS and the neutral phospholipid PE. It has been described before that positively charged histones bind the anionic phospholipid PS with high avidity (31). Specific phospholipid-dependent enhancement of APC activity has likewise been reported before. Part of this stimulatory effect will presumably be due to the fact that both the substrate (histone H3) and the enzyme (APC) bind to the phospholipid surface, promoting their interaction and reaction (32). For FVa inactivation it was described that optimal APC-catalyzed proteolysis requires calcium and PS containing phospholipid vesicles (30, 32, 33), moreover Smirnov et al. (34) and Norstrøm et al. (35) showed that PE has an essential role for optimal expression of the anticoagulant activity of APC in both purified systems and plasma.

We observed that 5A-APC, a non-anticoagulant engineered APC variant, cleaves histone H3 with significantly higher efficiency than wt-APC. 5A-APC is a variant wherein five positively charged amino-acids (lysine 191-193 and arginine 229-230) in an exosite of APC that is known to be important for electrostatic interaction with FVa, were mutated to alanine (36). We hypothesize that since histones are highly positively charged proteins, neutralization of the positively charged exosite has resulted in enhanced interaction of 5A-APC with histone $\mathrm{H} 3$, overall resulting in a more efficient histone $\mathrm{H} 3$ cleavage.

We identified, via N-terminal sequencing R26 and R52 as cleavage sites of APC in histone $\mathrm{H} 3$, resulting in end products K27-A135 (12.49 kDa) and R53-A135 (9.66 kDa). Using bioinformatics we had correctly predicted these fragments. Other predicted cleavage sites based on our algorhythm were R8/K9, R17, R128 and R131, resulting in the histone H3 fragments K18-R131 (13.04 kDa), S10-R131/K9-R131 (13.80/13.93 kDa) 
and A1-R128 (14.84 kDa). Peptidylarginine deiminase 4 (PAD4) citrullinates histone $\mathrm{H} 3$ residues R2, R8, R17 and R26 during NETosis (37, 38, 39). The degree of histone citrullination depends on local PAD4 and calcium concentrations and on the structure, charge and flexibility of amino acids flanking the arginine residues (40). Citrullination can theoretically increase susceptibility to proteolytic degradation by unfolding proteins. On the other hand citrullination can decrease proteolysis by loss of the positive charge of arginine residues (40). Due to these conflicting effects it is unknown what the net effect of histone citrullination on its proteolysis by APC will be.

We here confirm the existence of an APC-cleavage site at R26 cleavage site, as was already predicted by $\mathrm{Xu}$ et al. (4) for the highly homologous mouse histone $\mathrm{H} 3$ and provide evidence for an additional cleavage site at R52. At present we can only speculate on the relative importance of these two sites. However, we have shown that the 12.49 $\mathrm{kDa}$ and the $9.66 \mathrm{kDa}$ histone $\mathrm{H} 3$ fragments were not cytotoxic to endothelial cells, so cleavage of histones by APC at both sites can have potential therapeutic efficacy in patients suffering from acute and chronic pathologic conditions that are characterized by increased histone and nucleosome levels in circulation. We hypothesize that 5A-APC is superior to wt-APC as therapeutic agent to treat patients suffering from sepsis and traumatic injury because 5A-APC cleaves histones more efficiently and, perhaps even more importantly, 5A-APC exhibits only minimal anticoagulant activity, a feature of great value in patients at risk for bleeding. 


\section{References}

1. Mosnier L, Gale A, Yegneswaran S, Griffin J (2004) Activated protein C variants with normal cytoprotective but reduced anticoagulant activity. Blood 104: 1740-1744.

2. Luger K, Mäder A, Richmond R, Sargent D, Richmond T (1997) Crystal structure of the nucleosome core particle at 2.8 A resolution. Nature 389: 251-260.

3. Carruthers L, Bednar J, Woodcock C, Hansen J (1998) Linker histones stabilize the intrinsic saltdependent folding of nucleosomal arrays: mechanistic ramifications for higher-order chromatin folding. Biochemistry 37: 14776-14787.

4. Xu J, Zhang X, Pelayo R, Monestier M, Ammollo C, et al. (2009) Extracellular histones are major mediators of death in sepsis. Nat Med 15: 1318-1321.

5. Zeerleder S, Stephan F, Emonts M, de Kleijn E, Esmon C, et al. (2012) Circulating nucleosomes and severity of illness in children suffering from meningococcal sepsis treated with protein C. Crit Care Med 40: 3224-3229.

6. Zeerleder S, Zwart B, Wuillemin W, Aarden L, Groeneveld A, et al. (2003) Elevated nucleosome levels in systemic inflammation and sepsis. Crit Care Med 31: 1947-1951.

7. Chen Q, Ye L, Jin Y, Zhang N, Lou T, et al. (2012) Circulating nucleosomes as a predictor of sepsis and organ dysfunction in critically ill patients. Int J Infect Dis 16: e558-564.

8. Kutcher M, Xu J, Vilardi R, Ho C, Esmon C, et al. (2012) Extracellular histone release in response to traumatic injury: implications for a compensatory role of activated protein C. J Trauma Acute Care Surg 73: 1389-1394.

9. Fuchs T, Brill A, Duerschmied D, Schatzberg D, Monestier M, et al. (2010) Extracellular DNA traps promote thrombosis. PNAS 107: 15880-15885.

10. Brinkmann V, Reichard U, Goosmann C, Fauler B, Uhlemann Y, et al. (2004) Neutrophil extracellular traps kill bacteria. Science 303: 1532-1535.

11. Hirsch J (1958) Bactericidal action of histone. J Exp Med 108: 925-944.

12. Chaput C, Zychlinsky A (2009) Sepsis: the dark side of histones. Nat Med 15: 1245-1246.

13. Kleine $\mathrm{T}$, Lewis $\mathrm{P}$, Lewis $\mathrm{S}$ (1997) Histone-induced damage of a mammalian epithelium: the role of protein and membrane structure. Am J Physiol 273: C1925-1936.

14. Hariton-Gazal E, Rosenbluh J, Graessmann A, Gilon C, Loyter A (2003) Direct translocation of histone molecules across cell membranes. J Cell Sci 116: 4577-4586.

15. Rosenbluh J, Hariton-Gazal E, Dagan A, Rottem S, Graessmann A, et al. (2005) Translocation of histone proteins across lipid bilayers and Mycoplasma membranes. J Mol Biol 345: 387-400.

16. Xu J, Zhang X, Monestier M, Esmon N, Esmon C (2011) Extracellular histones are mediators of death through TLR2 and TLR4 in mouse fatal liver injury. J Immunol 187: 2626-2631.

17. Kalafatis M, Rand M, Mann K (1994) The mechanism of inactivation of human factor V and human factor Va by activated protein C. J Biol Chem 269: 31869-31880.

18. Fay P, Smudzin T, Walker F (1991) Activated protein C-catalyzed inactivation of human factor VIII and factor VIIIa. Identification of cleavage sites and correlation of proteolysis with cofactor activity. J Biol Chem 266: 20139-20145.

19. Gale A, Tsavaler A, Griffin J (2002) Molecular characterization of an extended binding site for coagulation factor Va in the positive exosite of activated protein C. J Biol Chem 277: 28836-28840.

20. Manithody C, Fay P, Rezaie A (2003) Exosite-dependent regulation of factor VIIIa by activated protein C. Blood 101: 4802-4807. 
21. Sun YH, Shen L, Dahlbäck B (2003) Gla domain-mutated human protein C exhibiting enhanced anticoagulant activity and increased phospholipid binding. Blood 101: 2277-2284.

22. Friedrich U, Pötzsch B, Preissner K, Müller-Berghaus G, Ehrlich H (1994) Calcium-dependent activation of protein $\mathrm{C}$ by thrombin/thrombomudulin: role of negatively charged amino acids within the activation peptide of protein C. Thromb Haemost 72: 567-572.

23. Nicolaes G, Bock P, Segers K, Wildhagen K, Dahlbäck B, et al. (2010) Inhibition of Thrombin Formation by Active Site Mutated (S360A) Activated Protein C. J Biol Chem 285: 22890-22900.

24. Nicolaes G, Villoutreix B, Dahlbäck B (1999) Partial glycosylation of Asn2181 in human factor V as a cause of molecular and functional heterogeneity. Modulation of glycosylation efficiency by mutagenesis of the consensus sequence for N-linked glycosylation. Biochemistry 38: 13584-13591.

25. Kalafatis M, Rand M, Mann K (1994) The mechanism of inactivation of human factor $V$ and human factor Va by activated protein C. J Biol Chem 269: 31869-31880.

26. Tran S, Dahlbäck B (2010) Novel APC-cleavage sites in FVa provide insights into mechanisms of action of APC and its cofactor protein S. J Thromb Haemost 8: 129-136.

27. Fay P, Smudzin T, Walker F (1991) Activated protein C-catalyzed inactivation of human factor VIII and factor VIIIa. Identification of cleavage sites and correlation of proteolysis with cofactor activity. J Biol Chem 266: 20139-20145.

28. Wildhagen K, Lutgens E, Loubele S, ten Cate H, Nicolaes G (2011) The structure-function relationship of activated protein C. Lessons from natural and engineered mutations. Thromb Haemost 106: 10341045.

29. Fürnrohr B, Groer G, Sehnert B, Herrmann M, Voll R (2007) Interaction of histones with phospholipids-implications for the exposure of histones on apoptotic cells. Autoimmunity 40: 322326.

30. Bakker H, Tans G, Janssen-Claessen T, Thomassen M, Hemker H, et al. (1992) The effect of phospholipids, calcium ions and protein $\mathrm{S}$ on rate constants of human factor Va inactivation by activated human protein C. Eur J Biochem 208: 171-178.

31. Pereira L, Marco F, Boimorto R, Caturla A, Bustos A, et al. (1994) Histones interact with anionic phospholipids with high avidity; its relevance for the binding of histone-antihistone immune complexes. Clin Exp Immunol 97: 175-180.

32. Nicolaes G, Tans G, Thomassen M, Hemker H, Pabinger I, et al. (1995) Peptide bond cleavages and loss of functional activity during inactivation of factor Va and factor VaR506Q by activated protein C. J Biol Chem 270: 21158-21166.

33. Rosing J, Hoekema L, Nicolaes G, Thomassen M, Hemker H, et al. (1995) Effects of protein S and factor Xa on peptide bond cleavages during inactivation of factor Va and factor VaR506Q by activated protein C. J Biol Chem 270: 27852-27858.

34. Smirnov M, Esmon C (1994) Phosphatidylethanolamine incorporation into vesicles selectively enhances factor Va inactivation by activated protein C. J Biol Chem 269: 816-819.

35. Norstrøm E, Steen M, Tran S, Dahlbäck B (2003) Importance of protein S and phospholipid for activated protein C-mediated cleavages in factor Va. J Biol Chem 278: 24904-24911.

36. Mosnier LO, Yang XV, Griffin JH (2007) Activated protein C mutant with minimal anticoagulant activity, normal cytoprotective activity, and preservation of thrombin activable fibrinolysis inhibitordependent cytoprotective functions. J Biol Chem 282: 33022-33033.

37. Sharma P, Azebi S, England P, Christensen T, Møller-Larsen A, et al. (2012) Citrullination of histone H3 interferes with HP1-mediated transcriptional repression. PLoS Genet 8: e1002934.

38. Leshner M, Wang S, Lewis C, Zheng H, Chen X, et al. (2012) PAD4 mediated histone hypercitrullination induces heterochromatin decondensation and chromatin unfolding to form neutrophil extracellular trap-like structures. Front Immunol 3. 
39. Wang Y, Li M, Stadler S, Correll S, Li P, et al. (2009) Histone hypercitrullination mediates chromatin decondensation and neutrophil extracellular trap formation. J Cell Biol 184: 205-213.

40. Vossenaar E, Zendman A, van Venrooij W, Pruijn G (2003) PAD, a growing family of citrullinating enzymes: genes, features and involvement in disease. Bioessays 25: 1106-1118. 


\section{Chapter 6}

\section{Extracellular histone $\mathrm{H} 3$ levels are associated with sepsis- induced organ failure and serve as predictive biomarkers of mortality in ICU sepsis patients}

Karin C.A.A. Wildhagen ${ }^{1}$, Maryse Wiewel' ${ }^{2}$, Marcus J. Schultz ${ }^{3}$, Janneke Horn ${ }^{3}$, Chris P.M. Reutelingsperger ${ }^{1}$, Tom van der Poll ${ }^{2}$, Gerry A.F. Nicolaes ${ }^{1}$

1. Department of Biochemistry, Cardiovascular Research Institute Maastricht (CARIM), Maastricht University, Maastricht, The Netherlands

2. Center for Experimental and Molecular Medicine (CEMM), Academic Medical Center, University of Amsterdam, Amsterdam, The Netherlands

3. Department of Intensive Care, Academic Medical Center, University of Amsterdam, Amsterdam, The Netherlands 


\section{Abstract}

Sepsis is a leading cause of death worldwide with an estimated incidence of at least 19 million cases per year and a mortality rate of 20-30\%. Extracellular histones are cytotoxic compounds mediating death in murine sepsis and circulating nucleosome levels predict mortality in human inflammation and sepsis.

In this study we measured plasma histone H3 (H3) levels in 43 sepsis patients and determined correlation with disease severity, organ failure, mortality and coagulationand tissue homeostasis parameters.

Median $\mathrm{H} 3$ levels were significantly increased in non-survivors compared to survivors 3.15 (IQR: 69) $\mu \mathrm{g} / \mathrm{ml}$, versus 0.57 (1.74) $\mu \mathrm{g} / \mathrm{ml}, \mathrm{P}=0.038$ ). H3 levels are positively correlated with lactate dehydrogenase $(\mathrm{LDH})$ activity (Pearson's $\mathrm{r}=0.873, \mathrm{P}=0.000$ ), lactate levels $(\mathrm{r}=0.403, \mathrm{P}=0.011)$ and Sequential Organ Failure Assessment (SOFA) score $(\mathrm{r}=0.373, \mathrm{P}=0.023)$ and negatively correlated with antithrombin levels $(\mathrm{r}=-0.347$, $\mathrm{P}=0.025)$ and platelet counts (rho $=-0.377, \mathrm{P}=0.013$ ).

We conclude that circulating $\mathrm{H} 3$ levels correlate with sepsis-induced organ failure and serve as a predictive biomarker of mortality in sepsis. 


\section{Introduction}

Sepsis is a syndrome caused by a deregulated host response to infection. Extrapolated from incidence rates in the United States, worldwide there are currently at least 19 million cases of sepsis per year (1). Despite advances in supportive therapy for organ failure and the use of potent antibiotics, mortality rates are still $20-30 \%$ for sepsis patients and even higher for severe sepsis and septic shock patients (2).

In murine models it was shown that extracellular histones are major mediators of death in sepsis and that mainly histones H3 and H4 are cytotoxic (3). Histones are highly alkaline nuclear proteins that form nucleosomes and, thus, organize the structure of chromatin. A nucleosome comprises two copies of histones H2A, H2B, H3 and H4 and is surrounded by a superhelix of 146 bp DNA (4). Histones can be released into the extracellular compartment during apoptosis and necrosis (5), as well as during NETosis $(6,7)$ where they cause endothelial dysfunction and eventually organ failure $(3$, 8). Predominantly $\mathrm{H} 3$ and $\mathrm{H} 4$ exhibit cytotoxic activity but the molecular mechanism underlying their cytotoxicity is still not fully understood. Histones can interact directly with phospholipid bilayers of cellular membranes, promote channel formation and cause loss of membrane barrier function (9-11). Alternatively, H3 and $\mathrm{H} 4$ can activate Toll-like receptors (TLR) 2 and 4 resulting in MyD88 signaling, NF-kB activation and downstream cytokine production, leukocyte recruitment, microvascular leakage, inflammation, tissue injury and finally death in murine models (12-14).

Elevated levels of circulating nucleosomes have been reported in patients with systemic inflammation and sepsis $(5,15,16)$ and correlate with mortality in traumatic injury patients (17). In addition, extracellular histones have been implicated in the pathogenesis of sepsis, thrombosis, peritonitis, lung injury, autoimmune diseases and brain, liver and kidney disease (18).

In this study we determined circulating $\mathrm{H} 3$ concentrations in the plasma of a group of 43 intensive care unit (ICU) sepsis patients. We determined correlations between $\mathrm{H} 3$ levels and mortality, disease severity and organ failure and between $\mathrm{H} 3$ levels and a number of variables reflecting coagulation status and tissue homeostasis. 


\section{Materials \& Methods}

\section{Study design}

43 sepsis patients admitted to the Intensive Care Unit (ICU) of the Academic Medical Center (AMC) in Amsterdam, The Netherlands, were included between May $1^{\text {st }}$ and December $31^{\text {th }} 2013$. The inclusion criteria were: age $>18$ years and a positive score for sepsis according to the Bone criteria (19): clinical suspicion of infection and at least 2 SIRS criteria: 1) a body temperature greater than $38^{\circ} \mathrm{C}$ or less than $36{ }^{\circ} \mathrm{C}$; 2) a heart rate greater than 90 beats/minute; 3) tachypnea, manifested by a respiratory rate greater than 20 breaths/minute or hyperventilation, as indicated by a $\mathrm{PaCO}_{2}$ of less than $32 \mathrm{mmHg}$; 4) an alteration in the white blood cell count, such as a count greater than $12.000 / \mathrm{mm}^{3}$, a count less than $4.000 / \mathrm{mm}^{3}$, or the presence of more than $10 \%$ immature neutrophils. The exclusion criteria were: no informed consent, patients receiving $>48$ hours of antibiotic treatment for a suspected infection prior to ICU admission and previous participation in this study. The group of sepsis patients was subdivided into sepsis, severe sepsis and septic shock patients. Severe sepsis patients meet the aforementioned criteria and in addition present with sepsis-induced organ dysfunction, hypoperfusion abnormality (lactic acidosis, oliguria, acute alteration of mental status) or hypotension. Septic shock patients have severe sepsis with hypotension despite adequate fluid resuscitation.

Upon ICU admission routine physiological measurements were performed and Acute Physiology and Chronic Health Evaluation III (APACHE III, range 0-299) and adjusted Sequential Organ Failure Assessment (SOFA) scores (range 0-20) were calculated. For calculation of the adjusted SOFA score, the scores that assess cardiovascular, coagulation, liver, renal and respiration systems were included. The central nervous system score was excluded, as this score is unreliable in sedated ICU patients. Within 24 hours of ICU admission arterial blood from each patient was withdrawn in vacutainer tubes containing 3.2\% citrate (BD, Franklin Lakes, New Jersey) and immediately centrifuged for $15 \mathrm{~min}$ at $1500 \mathrm{~g}$ at $4{ }^{\circ} \mathrm{C}$. Plasma samples were stored at $-80{ }^{\circ} \mathrm{C}$ to perform supplemental analyses at later time points. All laboratory tests were performed in a blind manner, without knowledge of the patient status. The study was approved by the Medical Ethics Review Committee of the AMC Amsterdam (NL 34294.018.10) and all participants or their representatives signed informed consent before enrollment in the study.

\section{Semi-quantitative $\mathrm{H} 3$ determination}

Ten times diluted plasma samples were separated via SDS-PAGE gel electrophoresis (615\%) and transferred to PVDF membranes (Pall Corporation, Port Washington, NY) by semi-dry blotting. After blocking, the membranes were overnight incubated at $4{ }^{\circ} \mathrm{C}$ with 
a rabbit polyclonal $\mathrm{H} 3$ antibody directed against an epitope at the C-terminus (1:1000, sc-8654-R, Santa Cruz Biotechnology, Heidelberg, Germany), followed by incubation with a biotinylated goat anti-rabbit $\operatorname{IgG}$ antibody (1:1000, Vector Laboratories, Burlingame, CA) for 30 minutes at RT and finally with StreptABComplex/AP solution (1:3000, Dako, Glostrup, Denmark) for 30 minutes at RT. Protein bands were detected by BCIP/NBT (Sigma-Aldrich, St. Louis, Missouri) and density of all H3 bands was quantified by ImageJ software (x86, Wayne Rasband). The densities of $\mathrm{H} 3$ bands in patient plasma samples were compared with the density of known concentrations of purified H3 (Roche, Basel, Switzerland) that were used as a reference to calculate H3 concentrations in patient samples.

\section{Calibrated Automated Thrombinography (CAT)}

Thrombin generation in clotting plasma was monitored by calibrated automated thrombinography (CAT), where conversion of a low affinity fluorogenic substrate (Z-Gly-Gly-Arg-7-amido-4-methylcoumarin (Bachem, Bubendorf, Switzerland)) by thrombin was followed over time in a Fluoroskan Ascent microtiter plate reader (Thermo Labsystems, Helsinki, Finland) with 390-nm excitation and 460-nm emission (20). CAT thrombin generation curves and the endogenous thrombin potential (ETP), were calculated using Thrombinoscope software (Thrombinoscope B.V., Maastricht, The Netherlands). Thrombin formation was routinely initiated by the addition of 5 $\mathrm{pM}$ of recombinant tissue factor, $16 \mathrm{mM} \mathrm{CaCl}_{2}, 30 \mu \mathrm{M}$ phospholipid vesicles (20:60:20 DOPS:DOPC:DOPE (mol:mol:mol)) and $50 \mu \mathrm{g} / \mathrm{ml}$ corn trypsin inhibitor. Pooled normal human plasma (34 donors: 14 females, 20 males), which was collected in the same way as the sepsis plasma samples, was used for normalization of measured values of the sepsis patients.

Patients that were on anticoagulant medication (acenocoumarol, heparin) before ICU admittance $(n=6)$, patients that received heparin at ICU before blood sampling $(n=5)$ and patients that received both anticoagulant medication before ICU admittance and heparin at ICU before blood sampling $(\mathrm{n}=4)$ were excluded from thrombin generation measurements.

\section{Lactate debydrogenase (LDH) activity assay}

Plasma samples were 25 times diluted in buffer (25 mm HEPES buffer, $150 \mathrm{~mm} \mathrm{NaCl}$, $5 \mathrm{mg} / \mathrm{ml}$ bovine serum albumin, $\mathrm{pH}$ 7.7) and a LDH activity assay kit (Sigma-Aldrich, St. Louis, Missouri) was used to measure $\mathrm{LDH}$ activity. 


\section{Antithrombin determination}

For the quantitative determination of antithrombin in plasma samples of sepsis patients the ACTICHROME ${ }^{\circledR}$ Antithrombin chromogenic activity kit (American Diagnostica Inc., Stamford, CT) was used. As describe before (21), thrombin was added to a plasma dilution containing antithrombin in the presence of excess heparin. After an initial incubation, residual thrombin was determined with a thrombin-specific chromogenic substrate. The residual thrombin activity was inversely proportional to the antithrombin concentration. Pooled normal human plasma (34 donors: 14 females, 20 males), which was collected in the same way as the sepsis plasma samples, was used as an antithrombin reference.

\section{Prothrombin determination}

Prothrombin concentrations in plasma samples of sepsis patients were determined using Ecarin (Pentapharm, Basel, Switzerland), a metalloprotease isolated from the venom of the saw-scaled viper (Echis carinatus), that specifically activates prothrombin independent of calcium, phospholipids and FXa/FV. Plasma samples were diluted in buffer ( $25 \mathrm{~mm}$ HEPES buffer, $150 \mathrm{~mm} \mathrm{NaCl}, 5 \mathrm{mg} / \mathrm{ml}$ bovine serum albumin, $5 \mathrm{mM}$ $\mathrm{CaCl}_{2}, \mathrm{pH} 7.7$ ), Ecarin was added (final concentration $0.33 \mathrm{U} / \mathrm{ml}$ ) and samples were incubated for 15 minutes at $37^{\circ} \mathrm{C}$. Next, samples were diluted in ice cold stopbuffer (20 $\mathrm{mM}$ Tris, $150 \mathrm{mM} \mathrm{NaCl}, 20 \mathrm{mM}$ EDTA, pH 7.9) and amidolytic thrombin activity was measured with the chromogenic substrate S-2238 (Chromogenix, Milan, Italy). Pooled normal human plasma (34 donors: 14 females, 20 males), which was collected in the same way as the sepsis plasma samples, was used as a normal prothrombin reference.

\section{Statistical analyses}

Analysis of data was performed with IBM SPSS Statistics 20. Normality of continuous variables was tested with the Shapiro-Wilk test. For nominal variables, significance of differences between groups was tested with the Fisher's exact test. For ordinal variables the non-parametric Kruskall Wallis and Mann Whitney U test were used to test for significance of difference between groups. To test for correlations between variables Pearson's correlation coefficient ( $r$ ) and Spearman's non parametric correlation coefficient rho where calculated. Both coefficients gave comparable results, so only Pearson's correlation coefficients are shown in Table 4. In all cases P-values $<0.05$ were regarded as statistically significant. 


\section{Results}

\section{Study population}

Baseline characteristics of the 43 sepsis patients included in the study are shown in Table 1. The percentages of males and females were comparable and most patients (91\%) were of Caucasian origin. The average age of the patients was 60 years and their average BMI was $27.9 \mathrm{~kg} / \mathrm{m}^{2}$. The study population had mean APACHE III and SOFA scores of respectively 83.7 and 9.7 and an average mortality rate of $28 \%$. The majority of patients had a primary infection of the respiratory tract (42\%), abdomen $(26 \%)$, cardiovascular system $(9 \%)$ or urinary tract $(9 \%)$. Infection was caused in $21 \%$ of cases by gram negative bacteria, in $9 \%$ of cases by gram positive bacteria and in $16 \%$ of cases by both gram negative and gram positive bacteria. In $51 \%$ of cases, the infection causative microorganism was not identified.

In Table 2 patients with sepsis, severe sepsis and septic shock are compared. Hospitalization time at the ICU, mortality rate, APACHE III score and SOFA score gradually increased with disease severity and were significantly increased in septic shock patients (all $\mathrm{P}=0.000$ ). In Table 3 characteristics of survivors and non-survivors of sepsis are shown. Median APACHE III and SOFA scores were respectively 73 and 8 in survivors of sepsis and were significantly increased to resp. 116 and 13 in non-survivors of sepsis $(\mathrm{P}=0.003$ and $\mathrm{P}=0.000)$.

\section{H3 levels, coagulation status and tissue homeostasis}

The majority of sepsis patients had plasma H3 levels below $10 \mu \mathrm{g} / \mathrm{ml}$, while 6 patients had higher H3 levels of 22, 64, 90, 93, 115 and $126 \mu \mathrm{g} / \mathrm{ml}$. H3 levels were comparable in sepsis, severe sepsis and septic shock patients (Figure 1A, Table 2). Median H3 levels were significantly increased from $0.57 \mu \mathrm{g} / \mathrm{ml}$ in non-survivors of sepsis to $3.15 \mu \mathrm{g} / \mathrm{ml}$ in survivors of sepsis $(\mathrm{P}=0.038$, Figure 1B, Table 3$)$. 
Table 1: Baseline characteristics of the study population

\begin{tabular}{|l|l|}
\hline & Average (range) or n (\%) \\
\hline Male, n (\%) & $24(56 \%)$ \\
\hline Age (years) & $60(19-92)$ \\
\hline BMI (kg/m $\mathbf{2})$ & $27.9(19.2-50.8)$ \\
\hline Race (white/black/asian), n (\%) & $39 / 3 / 1(91 / 7 / 2 \%)$ \\
\hline Time at ICU (days) & $6.5(2-36)$ \\
\hline Mortality at ICU (yes/no) & $12 / 31(28 / 72 \%)$ \\
\hline Sepsis/severe sepsis/septic shock, $\mathbf{n} \mathbf{( \% )}$ & $15 / 14 / 14(35 / 33 / 33 \%)$ \\
\hline APACHE III score & $83.7(26-153)$ \\
\hline SOFA score & $9.7(3-17)$ \\
\hline Primary site of infection, $\mathbf{n}(\mathbf{\%}):$ & \\
- respiratory tract & $43(100 \%)$ \\
- intra-abdominal & $18(42 \%)$ \\
- cardiovascular & $11(26 \%)$ \\
- urinary tract & $4(9 \%)$ \\
- skin or soft tissue & $4(9 \%)$ \\
- central nervous system & $3(7 \%)$ \\
- unknown & $1(2 \%)$ \\
\hline Primary cause of infection, $\mathbf{n}(\%):$ & $2(5 \%)$ \\
- gram negative bacteria & $43(100 \%)$ \\
- gram positive bacteria & $9(21 \%)$ \\
- gram negative + positive bacteria & $4(9 \%)$ \\
- yeasts or fungi & $7(16 \%)$ \\
- unknown & $1(2 \%)$ \\
\hline
\end{tabular}

The general characteristics of septic patients included in this study are shown, together with disease severity scores and mortality of patients. For all patients the primary site and cause of infection are shown. Abbreviations: APACHE: Acute Physiology and Chronic Health Evaluation; BMI: body mass index; ICU: intensive care unit; SOFA: Sequential Organ Failure Assessment.

In septic shock patients and non-survivors of sepsis a hypocoagulable state was observed. Septic shock patients had significantly increased PT $(\mathrm{P}=0.000)$, reduced platelet count $(\mathrm{P}=0.002)$ and decreased levels of prothrombin $(\mathrm{P}=0.017)$ and antithrombin $(\mathrm{P}=0.002)$ compared to sepsis patients. Non-survivors presented with significantly increased CAT lag time $(\mathrm{P}=0.044)$, decreased platelet count $(\mathrm{P}=0.020)$ and decreased antithrombin levels $(\mathrm{P}=0.014)$. In these patients a trend towards decreased prothrombin levels $(\mathrm{P}=0.096)$ and elevated PT $(\mathrm{P}=0.060)$ was observed. 
Table 2: Characteristics and biochemical data of septic patients

\begin{tabular}{|c|c|c|c|c|c|}
\hline \multirow[b]{2}{*}{ Variables } & \multirow{2}{*}{$\begin{array}{l}\begin{array}{c}\text { Sepsis, } \\
\mathrm{n}=15\end{array} \\
(34.9 \%)\end{array}$} & \multicolumn{2}{|c|}{$\begin{array}{c}\text { Severe sepsis, } \mathrm{n}=14 \\
(32.6 \%)\end{array}$} & \multicolumn{2}{|c|}{$\begin{array}{c}\text { Septic shock, } n=14 \\
(32.6 \%)\end{array}$} \\
\hline & & $\begin{array}{l}\text { Median } \\
\text { (IQR) }\end{array}$ & P-value & $\begin{array}{l}\text { Median } \\
\text { (IQR) }\end{array}$ & P-value \\
\hline Male, n (\%) & $12(80 \%)$ & $8(57 \%)$ & 0.245 & $4(29 \%)$ & $0.009 * *$ \\
\hline Age (years) ${ }^{\#}$ & $58(25)$ & $60(18)$ & 0.940 & $64(23)$ & 1.000 \\
\hline BMI $\left(\mathrm{kg} / \mathrm{m}^{2}\right)^{\#}$ & $24.7(7.8)$ & $31.3(9.6)$ & 0.061 & $25.1(8.0)$ & 0.568 \\
\hline Time at ICU (days) & $3(1)$ & $4.5(4)$ & $0.000^{* * *}$ & $6(8)$ & $0.000^{* * *}$ \\
\hline Mortality at ICU, n (\%) & $\mathbf{0}(0 \%)$ & $3(21 \%)$ & 0.100 & $9(64 \%)$ & $0.000^{* * *}$ \\
\hline APACHE III score & $57(35)$ & $67(20)$ & 0.161 & $117(27)$ & $0.000^{* * *}$ \\
\hline SOFA score ${ }^{\#}$ & $5.5(3)$ & $8.5(6)$ & $0.013^{*}$ & $13(4)$ & $0.000^{* * *}$ \\
\hline Histone $\mathrm{H} 3(\mu \mathrm{g} / \mathrm{ml})$ & $0.53(1.61)$ & $\mathbf{0 . 6 9}(7.05)$ & 0.159 & $1.95(9.36)$ & 0.179 \\
\hline Lactate $(\mathrm{mmol} / \mathrm{l})$ & $2.5(4.0)$ & $1.7(1.1)$ & 0.116 & $7.1(7.6)$ & $0.001 * *$ \\
\hline LDH activity (nmole/min/ml) & $259(162)$ & $353(232)$ & 0.227 & $464(620)$ & 0.056 \\
\hline CAT: lag time (ratio) & $1.56(0.62)$ & $1.95(0.47)$ & 0.210 & $1.70(1.53)$ & 0.730 \\
\hline CAT: ETP (ratio) & $1.33(0.59)$ & $1.49(0.71)$ & 0.586 & $1.02(0.66)$ & 0.503 \\
\hline $\begin{array}{l}\text { CAT: peak height (ratio) } \\
\text { (normalized) }^{\#}\end{array}$ & $1.31(0.52)$ & $1.32(0.37)$ & 0.940 & $1.10(1.59)$ & 0.666 \\
\hline Platelet count $\left(\times 10^{9} / \mathrm{l}\right)$ & $164.0(73.0)$ & $206.5(162.8)$ & 0.906 & $101.5(96.0)$ & $0.002 * *$ \\
\hline Prothrombin (\%) & $76(31)$ & $60.5(38)$ & $0.043^{*}$ & $54(42)$ & $0.017 *$ \\
\hline Antithrombin (\%) & $87(37)$ & $86(14)$ & 0.692 & $61(34)$ & $0.002 * *$ \\
\hline APTT (s) & $38(16)$ & $36(28)$ & 0.904 & $47(25)$ & 0.281 \\
\hline $\mathrm{PT}(\mathrm{s})$ & $12.1(1.6)$ & $16.0(6.9)$ & $0.042^{*}$ & $20.7(36.4)$ & $0.000^{* * *}$ \\
\hline
\end{tabular}

Sepsis patients were divided in patients with sepsis, severe sepsis (sepsis with sepsis-induced organ dysfunction) and septic shock (severe sepsis with hypotension despite adequate fluid resuscitation). For all continuous variables median and interquartile range (IQR) are shown. Variables with a normal distribution of data within all 3 groups are marked with \#. Significance of differences between the sepsis group and both other groups was calculated with the Fisher's exact test, the Kruskall Wallis test and posthoc Mann Whitney test. ${ }^{*} \mathrm{P}<0.05,{ }^{*} * \mathrm{P}<0.01,{ }^{*} * \mathrm{P}<0.001$. Abbreviations: APACHE: Acute Physiology and Chronic Health Evaluation; APTT: activated partial thromboplastin time; BMI: body mass index; CAT: calibrated automated thrombinography; ETP: endogenous thrombin potential; ICU: intensive care unit; LDH: lactate dehydrogenase; PT: prothrombin time; ratio: values of patients are normalized against healthy controls; SOFA: Sequential Organ Failure Assessment. 
Table 3: Characteristics and biochemical data of septic patients

\begin{tabular}{|c|c|c|c|}
\hline \multirow[b]{2}{*}{ Variables } & \multirow{2}{*}{\begin{tabular}{|c} 
Survivors, $\mathrm{n}=31$ \\
$(72.1 \%)$
\end{tabular}} & \multicolumn{2}{|c|}{$\begin{array}{c}\text { Non-survivors, } \mathrm{n}=12 \\
(27.9 \%)\end{array}$} \\
\hline & & Median (IQR) & P-value \\
\hline Male, n (\%) & $18(58 \%)$ & $6(50 \%)$ & 0.238 \\
\hline Age (years) & $58(21)$ & $65.5(14)$ & 0.129 \\
\hline BMI $\left(\mathrm{kg} / \mathrm{m}^{2}\right)$ & $26.1(8.9)$ & $27.4(9.8)$ & 0.899 \\
\hline Time at ICU (days) & $4(3)$ & $4.5(7)$ & 0.162 \\
\hline APACHE III score $\#$ & $73(32)$ & $116(54)$ & $0.003^{* *}$ \\
\hline SOFA score s $^{\#}$ & $8(4)$ & $13(3)$ & $0.000^{* * *}$ \\
\hline Histone $\mathrm{H} 3(\mu \mathrm{g} / \mathrm{ml})$ & $0.57(1.74)$ & $3.15(69.00)$ & $0.038^{*}$ \\
\hline Lactate $(\mathrm{mmol} / \mathrm{l})$ & $2.1(4.0)$ & $8.5(12.6)$ & $0.001^{* *}$ \\
\hline $\mathrm{LDH}$ activity (nmole/min/ml) & $269.5(179.0)$ & $\mathbf{5 5 1 . 5}(1978.0)$ & $0.001 * *$ \\
\hline CAT: lag time (ratio) & $1.58(0.81)$ & $1.95(1.19)$ & $0.044 *$ \\
\hline CAT: ETP (ratio) & $1.34(0.52)$ & $1.31(0.86)$ & 0.989 \\
\hline CAT: peak height (ratio) & $1.32(0.78)$ & $1.16(0.96)$ & 0.857 \\
\hline Platelet count $\left(\times 10^{9} / 1\right)^{\#}$ & $164(90)$ & $107.5(107)$ & $0.020^{*}$ \\
\hline Prothrombin $(\%)$ & $67(31)$ & $54(42)$ & 0.096 \\
\hline Antithrombin $(\%)^{\#}$ & $83(30)$ & $65.5(27)$ & $0.014^{*}$ \\
\hline APTT $(\mathrm{s})$ & $39(24)$ & $35(81)$ & 0.832 \\
\hline $\mathrm{PT}(\mathrm{s})$ & $14.1(7.7)$ & $17.1(19.8)$ & 0.060 \\
\hline
\end{tabular}

Sepsis patients were divided in survivors and non-survivors. Interquartile ranges (IQR) are shown for all continuous variables. Variables with a normal distribution of data within both groups are marked with \#. Significance of differences between both groups was calculated with the Fisher's exact test and Mann Whitney test. $* \mathrm{P}<0.05$, $* * \mathrm{P}<0.01$, $* * * \mathrm{P}<0.001$. Abbreviations: APACHE: Acute Physiology and Chronic Health Evaluation; APTT: activated partial thromboplastin time; BMI: body mass index; CAT: calibrated automated thrombinography; ETP: endogenous thrombin potential; ICU: intensive care unit; LDH: lactate dehydrogenase; PT: prothrombin time; ratio: values of patients are normalized against healthy controls; SOFA: Sequential Organ Failure Assessment.

To assess tissue homeostasis, lactate and LDH activity levels were measured. In septic shock patients maximum lactate levels measured within the first day of ICU admission were significantly increased $(\mathrm{P}=0.001)$ and a trend towards increased LDH activity levels was observed $(\mathrm{P}=0.056)$. Both $\mathrm{LDH}$ activity $(\mathrm{P}=0.001)$ and lactate $(\mathrm{P}=0.001)$ levels in plasma were significantly elevated in non-survivors compared to survivors of sepsis. 
A

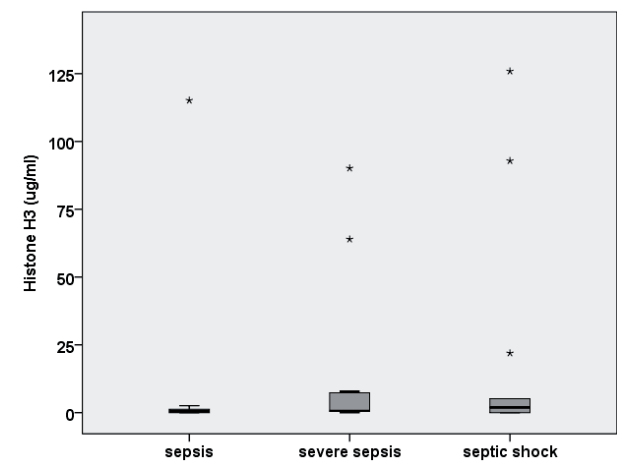

B

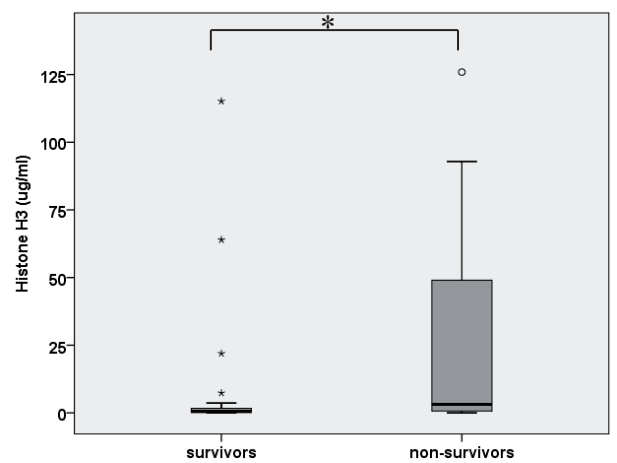

Figure 1: Histone $\mathrm{H} 3$ levels in sepsis patients

Boxplots with interquartile ranges are shown for histone $\mathrm{H} 3$ levels measured in (A) sepsis, severe sepsis and septic shock patients, (B) survivors and non-survivors of sepsis. Significance of differences is calculated with the Mann Whitney test, $* \mathrm{P}<0.05$.

\section{H3 levels correlate with LDH/lactate, platelet count, SOFA and antithrombin levels}

Table 4 shows significant correlations between H3 levels, disease severity scores and variables reflecting coagulation status and tissue homeostasis. We were most interested in correlations of $\mathrm{H} 3$ with other parameters, since little is known so far about possible associations between the presence of extracellular histones and other parameters. Significant correlations of H3 with LDH activity (Pearson's $r=0.873, \mathrm{P}=0.000$ ), lactate $(\mathrm{r}=0.403, \mathrm{P}=0.011)$, platelet count $(\mathrm{r}=-0.377, \mathrm{P}=0.013), \mathrm{SOFA}(\mathrm{r}=0.373, \mathrm{P}=0.023)$ and antithrombin levels $(\mathrm{r}=-0.347, \mathrm{P}=0.025)$ are shown in Figure 2 and highlighted in grey in Table 4. Additionally, there were correlations between disease severity scores, several coagulation parameters and lactate/LDH activity. Thus, increased levels of $\mathrm{H} 3$ in sepsis patients were associated with increased sepsis-related organ failure and with increased lactate/LDH activity and decreased antithrombin levels and platelet count. 

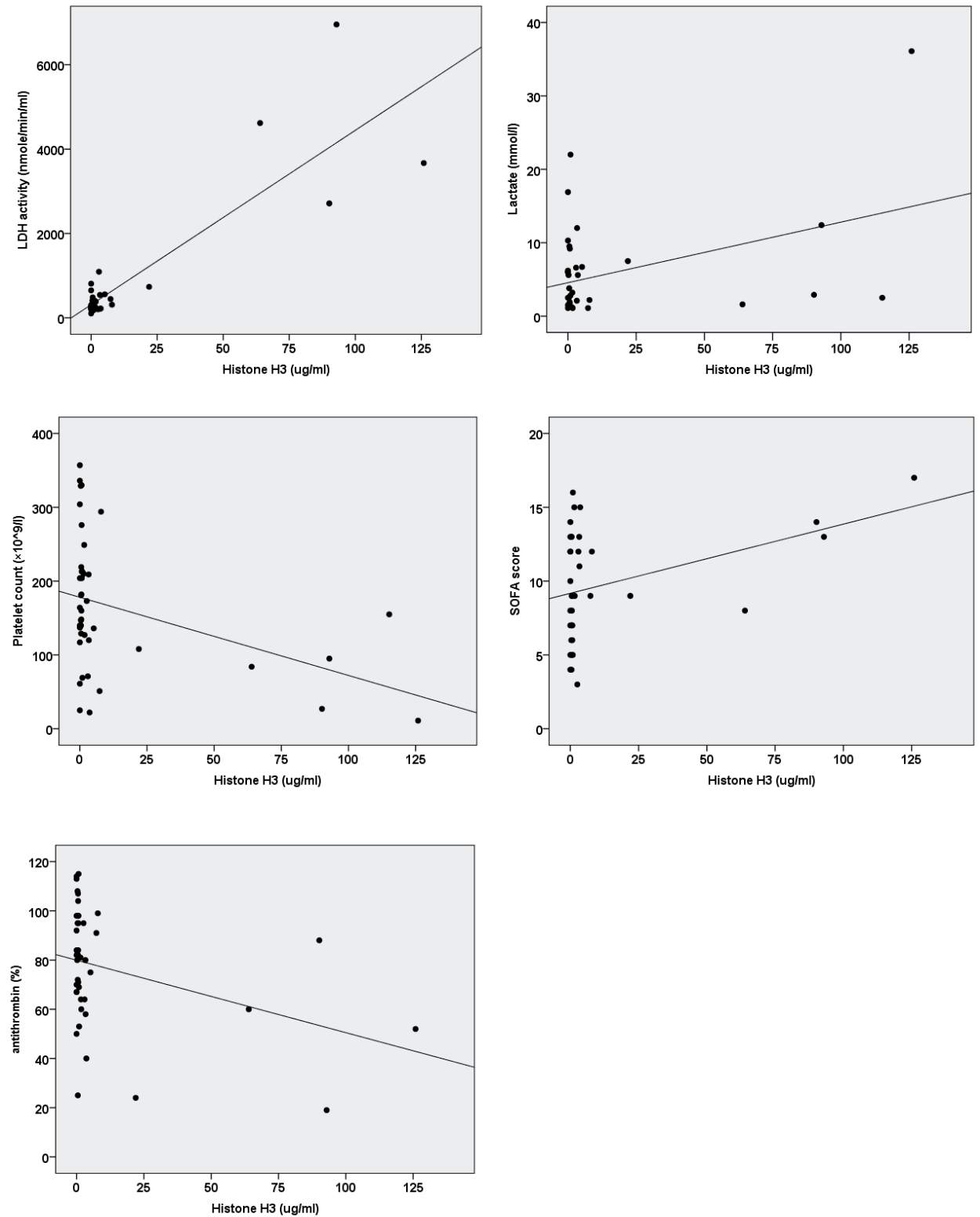

Figure 2: Correlations between histone $\mathrm{H} 3$ and other variables.

$\mathrm{XY}$ scatterplots and trendlines of significant correlations between histone H3 levels and other variables (see Table 4) are shown. 
Table 4: Significant correlations between pairs of variables measured in sepsis patients

\begin{tabular}{|l|l|l|l|l|}
\hline Variable 1 & Variable 2 & Pearson's r & P-value & N \\
\hline Histone H3 & LDH activity & 0.873 & 0.000 & 42 \\
\hline APACHE III score & SOFA score & 0.748 & 0.000 & 37 \\
\hline CAT: ETP & CAT: peak height & 0.738 & 0.000 & 25 \\
\hline Lactate & PT & 0.597 & 0.000 & 38 \\
\hline APACHE III score & Lactate & 0.567 & 0.000 & 39 \\
\hline SOFA score & Lactate & 0.547 & 0.001 & 34 \\
\hline SOFA score & Platelet count & -0.510 & 0.001 & 37 \\
\hline Lactate & Platelet count & -0.435 & 0.006 & 39 \\
\hline LDH activity & Antithrombin & -0.408 & 0.007 & 42 \\
\hline SOFA score & Antithrombin & -0.420 & 0.010 & 37 \\
\hline Histone H3 & Lactate & 0.403 & 0.011 & 39 \\
\hline Histone H3 & Platelet count & -0.377 & 0.013 & 43 \\
\hline LDH activity & Platelet count & -0.376 & 0.014 & 42 \\
\hline Antithrombin & Platelet count & 0.372 & 0.015 & 42 \\
\hline Antithrombin & Lactate & -0.382 & 0.018 & 38 \\
\hline APACHE III score & PT & 0.363 & 0.018 & 42 \\
\hline APACHE III score & Prothrombin & -0.357 & 0.020 & 42 \\
\hline Histone H3 & SOFA score & 0.373 & 0.023 & 37 \\
\hline Histone H3 & Antithrombin & -0.347 & 0.025 & 42 \\
\hline APACHE III score & Antithrombin & -0.344 & 0.026 & 42 \\
\hline LDH activity & Lactate & 0.358 & 0.027 & 38 \\
\hline SOFA score & PT & 0.364 & 0.029 & 36 \\
\hline APACHE III score & Platelet count & -0.324 & 0.034 & 43 \\
\hline
\end{tabular}

Pearson's correlation coefficient $(\mathrm{r})$ and P-values were calculated to test for significant correlations. A P-value < 0.05 was regarded as statistically significant. Abbreviations: APACHE: Acute Physiology and Chronic Health Evaluation; APTT: activated partial thromboplastin time; CAT: calibrated automated thrombinography; ETP: endogenous thrombin potential; LDH: lactate dehydrogenase; PT: prothrombin time; SOFA: Sequential Organ Failure Assessment. 


\section{Discussion}

In this study we determined associations between extracellular H3 levels, and disease severity and mortality in ICU sepsis patients. We also determined associations with biomarkers that reflect coagulation status and tissue homeostasis. We found extracellular H3 levels to correlate with mortality, sepsis-related organ failure, lactate/LDH activity, antithrombin levels and platelet count and we conclude that $\mathrm{H} 3$ levels may potentially be used as a novel marker to predict mortality in these patients.

In septic shock patients and non-survivors of sepsis, we observed a hypocoagulable state, characterized by decreased antithrombin and prothrombin levels, decreased platelet count, prolonged PT and CAT lag time, all pointing towards an upregulation of haemostatic processes and consumption of coagulation proteins. We confirm that non-survivors present with significantly increased CAT lag times and lactate/LDH levels and that septic shock patients have decreased platelet counts, as well as decreased prothrombin and antithrombin levels (22-28). Here we add the levels of circulating extracellular histones to these observations.

The hypocoagulable state in septic shock patients is preceded by systemic activation of the coagulation system and development of disseminated intravascular coagulation (DIC) is frequently observed. During DIC, small blood clots form inside blood vessels throughout the body, resulting in the consumption of platelets and coagulant and anticoagulant factors (29). At the same time, concentrations of anticoagulant proteins, like antithrombin, can be decreased due to impaired synthesis in the liver as a result of the acute phase response to inflammation and due to proteolytic degradation by elastases released by activated neutrophils (30).

In response to infection, activated neutrophils can form neutrophil extracellular traps (NETs) and can therefore, together with dying cells, become a source of extracellular histones in circulation. We observed a correlation between $\mathrm{H} 3$ levels and SOFA scores in ICU sepsis patients and significantly increased H3 levels in plasma of non-survivors compared to survivors of sepsis. Extracellular histones possess direct cytotoxic effects by formation of pores in cell membranes (9-11) and can activate NLRP3 inflammasome (31), leading to caspase-1-dependent pro-inflammatory cytokine secretion, controlling host responses to infection and injury (32). Furthermore circulating histones can induce TLR2- and 4-dependent MyD88 signaling in immune cells and platelets, resulting in activation of NF-KB and MAPK target genes and downstream cytokine production, leukocyte recruitment, endothelial cell death, microvascular leakage, inflammation, thrombin generation, platelet aggregation and clotting, and as a consequence cause tissue injury and death in the context of sepsis and DIC (3,12-14,33-35).

Formation of (micro)thrombi and fibrin deposition in blood vessels can lead to hypoxia and necrosis of organ tissue and plays a pathologic role in the development of organ failure and subsequent mortality in sepsis (29). Due to hypoxia, organs have to increase their anaerobic glycolysis, resulting in increased lactate production. Other causes for 
increased lactate/LDH levels can be increased aerobic glycolysis and a decreased clearance of lactate by the liver $(36,37)$. Previous research $(22,27,28)$ has shown that increased lactate levels or the failure to clear lactate or LDH within the first 24-48 hours after ICU admittance are strong predictors of mortality in sepsis patients, in agreement with the significantly increased lactate/LDH activity levels observed in non-survivors $(\mathrm{P}=0.001)$, compared to survivors of sepsis in our study.

The development and progression of sepsis is a multi-factorial process. We show that extracellular histone levels, lactate/LDH activity and coagulation parameters in sepsis patients all reflect ongoing systemic inflammation, coagulation and organ failure and the extent of these can be used to estimate organ failure and predict outcome in sepsis patients. While therapy aimed at improvement of organ function, and restoration of the normal haemostatic balance are already being used, recent development from our laboratory and those of others $(3,6,12-14,34,35,38,39)$ have indicated that also the targeting of extracellular histones may provide cytoprotection to patients whose organ functions are at risk.

\section{Acknowledgement}

The support by a grant of the Cardiovascular Research Institute Maastricht (CARIM, to G.N.) is greatly acknowledged. We want to thank Ton Anbergen (Department of Methodology \& Statistics, Maastricht University, The Netherlands) for assistance with the statistical analyses performed. 


\section{References}

1. Adhikari N, Fowler R, Bhagwanjee S, Rubenfeld G (2010) Critical care and the global burden of critical illness in adults. Lancet 376: 1339-1346.

2. Angus D, van der Poll T (2013) Severe sepsis and septic shockvere sepsis and septic shock. N Engl J Med 369: 840-851.

3. Xu J, Zhang X, Pelayo R, Monestier M, Ammollo C, et al. (2009) Extracellular histones are major mediators of death in sepsis. Nat Med 15: 1318-1321.

4. Luger K, Mäder A, Richmond R, Sargent D, Richmond T (1997) Crystal structure of the nucleosome core particle at 2.8 A resolution. Nature 389: 251-260.

5. Zeerleder S, Zwart B, Wuillemin W, Aarden L, Groeneveld A, et al. (2003) Elevated nucleosome levels in systemic inflammation and sepsis. Crit Care Med 31: 1947-1951.

6. Fuchs T, Brill A, Duerschmied D, Schatzberg D, Monestier M, et al. (2010) Extracellular DNA traps promote thrombosis. PNAS 107: 15880-15885.

7. Brinkmann V, Reichard U, Goosmann C, Fauler B, Uhlemann Y, et al. (2004) Neutrophil extracellular traps kill bacteria. Science 303: 1532-1535.

8. Chaput C, Zychlinsky A (2009) Sepsis: the dark side of histones. Nat Med 15: 1245-1246.

9. Kleine T, Lewis P, Lewis S (1997) Histone-induced damage of a mammalian epithelium: the role of protein and membrane structure. Am J Physiol 273: C1925-1936.

10. Hariton-Gazal E, Rosenbluh J, Graessmann A, Gilon C, Loyter A (2003) Direct translocation of histone molecules across cell membranes. J Cell Sci 116: 4577-4586.

11. Rosenbluh J, Hariton-Gazal E, Dagan A, Rottem S, Graessmann A, et al. (2005) Translocation of histone proteins across lipid bilayers and Mycoplasma membranes. J Mol Biol 345: 387-400.

12. Xu J, Zhang X, Monestier M, Esmon N, Esmon C (2011) Extracellular histones are mediators of death through TLR2 and TLR4 in mouse fatal liver injury. J Immunol 187: 2626-2631.

13. Allam R, Scherbaum C, Darisipudi M, Mulay S, Hagele H, et al. (2012) Histones from dying renal cells aggravate kidney injury via TLR2 and TLR4. J Am Soc Nephrol 23: 1375-1388.

14. Semeraro F, Ammollo C, Morrissey J, Dale G, Friese P, et al. (2011) Extracellular histones promote thrombin generation through platelet-dependent mechanisms: involvement of platelet TLR2 and TLR4. Blood 118: 1952-1961.

15. Zeerleder S, Stephan F, Emonts M, de Kleijn E, Esmon C, et al. (2012) Circulating nucleosomes and severity of illness in children suffering from meningococcal sepsis treated with protein C. Crit Care Med 40: 3224-3229.

16. Chen Q, Ye L, Jin Y, Zhang N, Lou T, et al. (2012) Circulating nucleosomes as a predictor of sepsis and organ dysfunction in critically ill patients. Int J Infect Dis 16: e558-564.

17. Kutcher M, Xu J, Vilardi R, Ho C, Esmon C, et al. (2012) Extracellular histone release in response to traumatic injury: implications for a compensatory role of activated protein C. J Trauma Acute Care Surg 73: 1389-1394.

18. Allam R, Kumar S, Darisipudi M, Anders H (2014) Extracellular histones in tissue injury and inflammation. J Mol Med (Berl) 92: 465-472.

19. Bone R, Balk R, Cerra F, Dellinger R, Fein A, et al. (1992) Definitions for sepsis and organ failure and guidelines for the use of innovative therapies in sepsis. The ACCP/SCCM Consensus Conference Committee. American College of Chest Physicians/Society of Critical Care Medicine. Chest 101: 1644-1655. 
20. Hemker H, Giesen P, Dieri RA, Regnault V, Smedt Ed (2002) The calibrated automated thrombogram (CAT): a universal routine test for hyper- and hypocoagulability. Pathophysiol Haemost Thromb 32: 249-253.

21. Odegard O, Lie M, Abildgaard U (1975) Heparin cofactor activity measured with an amidolytic method. Thromb Res 6: 287-294.

22. Zein J, Lee G, Tawk M, Dabaja M, Kinasewitz G (2004) Prognostic Significance of Elevated Serum Lactate Dehydrogenase (LDH) in Patients with Severe Sepsis. Chest 126: 873S.

23. Van Dreden P, Woodhams B, Rousseau A, Dreyfus J, Vasse M (2013) Contribution of procoagulant phospholipids, thrombomodulin activity and thrombin generation assays as prognostic factors in intensive care patients with septic and non-septic organ failure. Clin Chem Lab Med 51: 387-396.

24. Collins P, Macchiavello L, Lewis S, Macartney N, Saayman A, et al. (2006) Global tests of haemostasis in critically ill patients with severe sepsis syndrome compared to controls. Br J Haematol 135: 220-227.

25. Petros S, Kliem P, Siegemund T, Siegemund R (2012) Thrombin generation in severe sepsis. Thromb Res 129: 797-800.

26. Massion P, Peters P, Ledoux D, Zimermann V, Canivet J, et al. (2012) Persistent hypocoagulability in patients with septic shock predicts greater hospital mortality: impact of impaired thrombin generation. Intensive Care Med 38: 1326-1335.

27. Marty P, Roquilly A, Vallée F, Luzi A, Ferré F, et al. (2013) Lactate clearance for death prediction in severe sepsis or septic shock patients during the first 24 hours in Intensive Care Unit: an observational study. Ann Intensive Care 3.

28. Londoño J, León A, Rodríguez F, Barrera L, de la Rosa G, et al. (2013) Serum lactate in the emergency department as a prognostic factor in patients with sepsis without hypotension. Med Clin (Barc) 141: 246-251.

29. Levi M, de Jonge E, van der Poll T (2003) Sepsis and disseminated intravascular coagulation. J Thromb Thrombolysis 16: 43-47.

30. Levi M, Van der Poll T (2005) Two-way interactions between inflammation and coagulation. Trends Cardiovasc Med 15: 254-259.

31. Allam R, Darisipudi M, Tschopp J, Anders H (2013) Histones trigger sterile inflammation by activating the NLRP3 inflammasome. Eur J Immunol 43: 3336-3342.

32. Schroder K, Tschopp J (2010) The inflammasomes. Cell 140: 821-832.

33. Carestia A, Rivadeneyra L, Romaniuk M, Fondevila C, Negrotto S, et al. (2013) Functional responses and molecular mechanisms involved in histone-mediated platelet activation. Thromb Haemost 110: 1035-1045.

34. Fuchs T, Bhandari A, Wagner D (2011) Histones induce rapid and profound thrombocytopenia in mice. Blood 118: 3708-3714.

35. Wildhagen K, García de Frutos P, Reutelingsperger C, Schrijver R, Aresté C, et al. (2014) Nonanticoagulant heparin prevents histone-mediated cytotoxicity in vitro and improves survival in sepsis. Blood 123: 1098-1101.

36. Phypers B, Pierce J (2006) Lactate physiology in health and disease. Anaesthesia, Critical Care \& Pain 6: $128-132$.

37. Gibot S (2012) On the origins of lactate during sepsis. Crit Care 16: 151.

38. Saffarzadeh M, Juenemann C, Queisser M, Lochnit G, Barreto G, et al. (2012) Neutrophil extracellular traps directly induce epithelial and endothelial cell death: a predominant role of histones. PLoS One 7: e32366.

39. Ammollo C, Semeraro F, Xu J, Esmon N, Esmon C (2011) Extracellular histones increase plasma thrombin generation by impairing thrombomodulin-dependent protein $\mathrm{C}$ activation. J Thromb Haemost 9: 1795-1803. 



\section{Chapter 7}

\section{Nonanticoagulant heparin prevents histone-mediated cytotoxicity in vitro and improves survival in sepsis}

Karin C. A. A. Wildhagen ${ }^{1 *}$, Pablo García de Frutos ${ }^{2 *}$, Chris P. Reutelingsperger ${ }^{1}$, Roy Schrijver ${ }^{1}$, Cristina Aresté ${ }^{2}$, Almudena Ortega-Gómez ${ }^{3}$, Niko M. Deckers ${ }^{1}$, H. Coenraad Hemker ${ }^{4}$, Oliver Soehnlein ${ }^{3,5,6}$ and Gerry A. F. Nicolaes ${ }^{1}$

*These authors contributed equally to this work.

1. Department of Biochemistry, Cardiovascular Research Institute Maastricht, Maastricht University, Maastricht, The Netherlands

2. Department of Cell Death and Proliferation, Institute of Biomedical Research of Barcelona, Barcelona, Spain

3. Institute for Cardiovascular Prevention, Ludwig-Maximilians-University Munich, Munich, Germany

4. Synapse BV, Cardiovascular Research Institute Maastricht, Maastricht University, Maastricht, The Netherlands

5. Department of Pathology, Academic Medical Center of the University of Amsterdam, Amsterdam, The Netherlands

6. German Centre for Cardiovascular Research, Partner Site Munich Heart Alliance, Munich, Germany 


\section{Abstract}

Extracellular histones are considered to be major mediators of death in sepsis. Although sepsis is a condition that may benefit from low-dose heparin administration, medical doctors need to take into consideration the potential bleeding risk in sepsis patients who are already at increased risk of bleeding due to a consumption coagulopathy. Here, we show that mechanisms that are independent of the anticoagulant properties of heparin may contribute to the observed beneficial effects of heparin in the treatment of sepsis patients. We show that nonanticoagulant heparin, purified from clinical grade heparin, binds histones and prevents histone-mediated cytotoxicity in vitro and reduces mortality from sterile inflammation and sepsis in mouse models without increasing the risk of bleeding. Our results demonstrate that administration of nonanticoagulant heparin is a novel and promising approach that may be further developed to treat patients suffering from sepsis. 


\section{Introduction}

Sepsis and septic shock are serious clinical problems with high mortality rates for which no adequate treatment currently exists (1). Neutrophils respond to infection with the formation of neutrophil extracellular traps (NETs) $(2,3)$ intricate networks containing DNA as the major structural component and proteins like histones and neutrophil elastase, which have antimicrobial properties. Extracellular histones, however, also exhibit cytotoxic activity toward host cells, including the endothelium $(4,5)$ Histone release can thus trigger a feedback cascade, resulting in more cell death and additional release of histones (6). Consequently, extracellular histones are considered interesting therapeutic targets for sepsis treatment (4).

Histones are positively charged, and NET-mediated cytotoxicity can be reduced with polysialic acid, a negatively charged polymer (5). We hypothesized that heparin, a negatively charged polysaccharide, blocks histone cytotoxicity and reduces mortality from sterile inflammation and sepsis. Low dose unfractionated heparin (UFH) has been tested in a clinical trial as a complementary treatment of sepsis (7). The study rationale linked infection, inflammation, and coagulation in sepsis and sought to inhibit the coagulation part with low doses of heparin so as not to increase the risk of bleeding in a patient who is already at risk due to sepsis-associated consumption coagulopathy (7, 8). Nevertheless, although this study failed to demonstrate a significant benefit on 28day mortality rate, we hypothesize that the minor beneficial effects of heparin observed might be attributed to a mechanism independent of the anticoagulant properties of heparin. We reasoned that removing the anticoagulant fraction from UFH would yield an antithrombin affinity-depleted heparin (AADH) that neutralizes histone-mediated cytotoxicity and effectively treats sepsis without increasing risk of bleeding. Heparins have the highest negative charge density of any known biological molecule (9) and have a strong affinity for histones $(10,11)$. It is, however, not known whether binding of heparin to histones also protects against the cytotoxic effect of histones on endothelial cells and/or in vivo. Here, we investigated the effects of UFH and AADH on histone cytotoxicity. We present data showing that complex formation of histones with heparin reduces their cytotoxicity in an in vitro cell-based cytotoxicity assay. Furthermore, by use of in vivo mouse models of sterile inflammation, a cecal ligation and puncture (CLP) model for sepsis and bacterial lipopolysaccharide (LPS) challenge, we show that AADH increases the survival rate in all 3 animal models.

\section{Study design}

AADH was produced by fractionation of UFH via affinity chromatography using an antithrombin-column, and its anticoagulant properties were quantitated via calibrated automated thrombography (CAT). The cytotoxic effects of purified histones, in the 
presence of UFH and AADH, were tested in vitro via a flow cytometry-based histone cytotoxicity assay employing EA.hy926 cells. Direct binding interaction between histones and $\mathrm{AADH}$ was determined using surface plasmon resonance and confocal laser scanning fluorescence microscopy. In vivo, the activities of UFH and AADH were tested in C57B16/J mice through a concanavalin A (ConA) challenge model for fatal sterile inflammation, through a CLP model and an LPS challenge model. Survival was used as the primary endpoint in these assays; liver viability assessment was performed to correlate disease severity to organ damage in the animals tested (CLP) and neutrophil lung infiltration, tissue leakage, and bronchoalveolar lavage cytokine levels were determined in the LPS model. Anticoagulant properties of administered UFH and AADH were assessed via CAT and tail bleeding time measurements. The experiments were approved by the animal care committees of the Spanish National Research Council and of the Ludwig-Maximilians-University Munich.

\section{Materials and Methods}

\section{Collection and handling of mouse plasma samples}

After intraperitoneal (i.p.) injection with urethane, the abdominal cavity of C57BL6/J mice was opened and cardiac puncture was performed. Blood was drawn into a syringe containing acid-citrate-dextrose (ACD) buffer (9:1 volume). Plasma was prepared by centrifugation, twice at $800 \mathrm{xg}$ for 10 minutes at RT after which the supernatant plasma was collected. As a reference plasma samples of healthy untreated C57BL6/J mice were pooled: control plasma pool $(\mathrm{n}=6)$, AADH3 $(\mathrm{n}=6)$, SAL3 $(\mathrm{n}=5)$, AADH6 $(\mathrm{n}=6)$, SAL6 $(\mathrm{n}=6)$.

\section{Production of $A A D H$}

Heparin sodium salt (2 mg per run, Sigma-Aldrich, St. Louis, MO, USA) was dissolved in running buffer $(140 \mathrm{mM} \mathrm{NaCl}, 20 \mathrm{mM}$ Tris, $\mathrm{pH}$ 7.4) and loaded onto a $5 \mathrm{ml}$ HiTrap NHS-activated HP column (GE Healthcare, Little Chalfont, UK) to which $12 \mathrm{mg}$ of bovine antithrombin had been covalently immobilized. Antithrombin affinity depleted heparin $(\mathrm{AADH})$ did not bind to the column and was found in the flow through. The AADH was then concentrated and switched to $140 \mathrm{mM} \mathrm{NaCl}, 20 \mathrm{mM}$ Tris, $\mathrm{pH} 7.4$ buffer by use of a PD-10 column (GE Healthcare, Little Chalfont, UK) and filtered over a $0.2 \mu \mathrm{M}$ filter (Whatman, Maidstone, UK). We monitored the purification by thrombin generation assay, described below, and we have selected those fractions that showed no significant effect on aPTT or anti-FXa levels. 


\section{Thrombin generation}

Thrombin generation was measured in triplicate via calibrated automated thrombography (CAT) essentially as described before (12). Thrombin generation in mouse plasma was performed as previously described (13) in the presence of varying concentrations of $\mathrm{UFH}$ and $\mathrm{AADH}$.

\section{Cell culture}

The permanent human endothelial cell line EA.hy926 was kindly provided by Dr. Dariusz Lezczynski (STUK - Radiation and Nuclear Safety Authority, Helsinki, Finland). EA.hy926 were cultured as described (14).

\section{Histone cytotoxicity assay}

EA.hy926 (1 x $10^{5}$ cells/well) were grown to confluence. Cells were washed twice with PBS and next incubated for 1 hour at $37^{\circ} \mathrm{C}$ in serum-free DMEM containing $40 \mu \mathrm{g} /$ $\mathrm{ml}$ histone H3 (Roche Applied Science, Penzberg, Germany; $100 \mu \mathrm{g} / \mathrm{ml}$ histone mix, Sigma-Aldrich, St. Louis, MO, USA was equally active) in combination with varying concentrations of UFH (LEO Pharma BV, Amsterdam, The Netherlands) or AADH. After 1 hour incubation the cells were washed and detached using $0.05 \%$ trypsin-EDTA. The cells were diluted to $1 * 10^{6}$ cells $/ \mathrm{ml}$ in $10 \mathrm{mM}$ Hepes, $150 \mathrm{mM} \mathrm{NaCl}, 5 \mathrm{mM} \mathrm{KCl}$, $1 \mathrm{mM} \mathrm{MgCl}$ and $2.5 \mathrm{mM} \mathrm{CaCl}_{2} \mathrm{pH} 7.4$ buffer containing $0.25 \mu \mathrm{g} / \mathrm{ml}$ Annexin A5FITC (PharmaTarget BV, Maastricht, The Netherlands), $2.5 \mu \mathrm{g} / \mathrm{ml}$ propidium iodide (Invitrogen Life Technologies, Carlsbad, CA, USA) and incubated for 10 minutes in the dark on ice. Cell viability was measured by flow cytometry on an Epics XL MCL, Beckman coulter flow cytometer, using FL1 PMT at 505-545 nm for FITC and FL3 PMT at 605-635 nm for propidium iodide.

\section{Western Blotting and precipitation}

Samples were denatured in standard gel sample buffer containing $1.25 \%$ mercaptoethanol at $95^{\circ} \mathrm{C}$ for 10 minutes, separated via electrophoresis in $15 \%$ SDS-polyacrylamide gels and transferred to PVDF membranes (Pall Life Sciences, Pensacola, FL, USA) by semi-dry blotting. After blocking the membranes were incubated overnight at $4^{\circ} \mathrm{C}$ with primary antibody for histone H3 (1:1000, Santa Cruz Biotechnology, Santa Cruz, CA, USA), followed by incubation with biotin-conjugated secondary antibody (1:1000, Vector Laboratories, Burlingame, CA, USA) for 30 minutes at RT and finally incubated with StreptABComplex/AP solution (1:3000, Dako Cytonation, Glostrup, Denmark) for 30 minutes at RT. Protein bands were detected by BCIP/NBT (Sigma-Aldrich, Steinheim, Germany) and histone H3 density was quantified by ImageJ software (x86, 
Wayne Rasband, National Institutes of Health, USA). For precipitation of the AADHhistone complex, $100 \mu \mathrm{g}$ histone $\mathrm{H} 3$ was mixed with $57 \mu \mathrm{g}$ AADH and incubated at $4^{\circ} \mathrm{C}$ for 30 minutes, next the reaction volume was centrifuged for $5 \mathrm{~min}$ at 20,000g and the pellet and supernatant were separately collected.

\section{Surface Plasmon resonance}

Histone $\mathrm{H} 3$ was coupled to a CM5 sensor chip using HBS-EP (10mM HEPES, pH 7.4, $150 \mathrm{mM} \mathrm{NaCl}, 3 \mathrm{mM}$ EDTA, $0.005 \%$ Tween-20) buffer at $25^{\circ} \mathrm{C}$. Histone $\mathrm{H} 3$ was diluted in $10 \mathrm{mM}$ Sodium-acetate buffer $\mathrm{pH} 5.0$ to a concentration of $50 \mu \mathrm{g} / \mathrm{ml}$ and immobilized to the chip with $\sim 4400$ response units.

SPR binding experiments were performed at $25^{\circ} \mathrm{C}$ at a flow rate of $50 \mu \mathrm{l} / \mathrm{min}$ and using HBSEP as running buffer. A single-cycle kinetics experiment was done with 5 different concentrations of $\operatorname{AADH}(100,50,10,5$ and $1 \mathrm{nM})$, an association time of $300 \mathrm{sec}$ and a final dissociation time of $1200 \mathrm{sec}$.

\section{Cytokine measurement}

Cytokine levels of IL-1 $\alpha$, IL-1 $\beta$, IL-2, IL-4, IL-6, IL-10, IL-12p70, IFN $\gamma$ and TNF were determined in mouse plasma samples by mouse cytokine IR array (9-plex) (Quansys biosciences, Logan, UT, USA).

\section{Concanavalin A (ConA) sterile inflammation model}

Eight to twelve weeks old C57B16/J mice were injected i.p. with a single dose of $570 \mu \mathrm{g}$ AADH or buffer and after 60 minutes received an intravenous injection of $30 \mathrm{mg} / \mathrm{kg}$ ConA, for the following 24 hours the condition of mice was continuously monitored, while survival as final parameter was registered.

\section{Cecal ligation and puncture (CLP), prophylactic regimen}

Mice were anesthetized with an i.p. injection of ketamine (125 mg/kg body weight (b.w.); Sanofi-Cefa GmbH Düsseldorf, Germany) and xylazine (12.5 mg/kg b.w.; Phoenix Scientific, Encinitas, CA, USA). After positioning on a heating plate, longitudinal skin midline incision was performed and abdominal cavity was opened in the midline. After identifying the cecum, cecum was ligated and punctured with a $22 \mathrm{G}$ needle. Fascia, abdominal musculature and skin were closed by applying simple running sutures. Mice were treated with $570 \mu \mathrm{g}$ AADH or buffer by i.p. injection 12 hours before CLP, right after CLP, and 12 hours after CLP. 


\section{Cecal ligation and puncture (CLP), treatment regimen}

Mice were anesthetized and treated as described for the prophylactic regimen above. Treatment was performed once with $570 \mu \mathrm{g}$ AADH or buffer by i.p. injection 4 hours post CLP. Group size is: sham: $n=15$, CLP group, no treatment n=15; CLP group + AADH treatment $\mathrm{n}=10$.

\section{Isolation and activation of neutrophils}

Human neutrophils were isolated from a healthy donor using density gradient separation with histopaque-1077. Cells were resuspended in phenol-red free RPMI 1640 medium $\left(1.75 \times 10^{6} \mathrm{cell} / \mathrm{s} / \mathrm{ml}\right)$ and seeded onto glass coverslips in 12 well tissue culture plates. To induce NETosis cells were stimulated with $50 \mathrm{nM}$ PMA for 2 hours at $37^{\circ} \mathrm{C}$.

\section{Scanning electron microscopy (SEM)}

After incubation with PMA, neutrophils on glass coverslips were fixed overnight with $2.5 \%$ glutaraldehyde at $4^{\circ} \mathrm{C}$. The specimens were postfixed using $1 \%$ osmiumtetroxide and dehydrated in graded ethanol series. For SEM, the specimens were dehydrated, critical point dried, mounted with silver paint on specimen tubs, and coated with gold. The surface of the membrane was examined in a SEM (Philips CM100 electron microscope) at $10 \mathrm{kV}$.

\section{Labeling of AADH with fluorescein (FITC)}

$\mathrm{AADH}(70 \mu \mathrm{M})$ was incubated with $0.28 \mathrm{M}$ 5-(((2(carbohydrazino)methyl)thio) acetyl) aminofluorescein (Invitrogen Life Technologies, Carlsbad, CA, USA) and 0.9 $\mathrm{M} \mathrm{NaCNBH}$ in acetate buffer in the dark for $4 \mathrm{~h}$ at $37^{\circ} \mathrm{C}$ followed by 2 days at RT. Afterwards the AADH-FITC was dialyzed in a slide-A-lyzer dialysis cassette with a MWCO of $2 \mathrm{kDa}$ (Thermo scientific, Rockford, IL, USA) at least four times against buffer (140 mM NaCl, $20 \mathrm{mM}$ Tris, $\mathrm{pH}: 7.4)$ to get rid of unbound label.

\section{Fluorescence microscopy}

After incubation with PMA, cells on glass coverslips were washed with PBS, fixed with 4\% PFA for $15 \mathrm{~min}$ at RT and incubated at RT for respectively 30 min with AADHFITC, for $2.5 \mathrm{~h}$ with rabbit monoclonal anti-histone H2B clone EP957Y (Millipore, Billerica, MA, USA), for $1 \mathrm{~h}$ with biotinylated Goat Anti-Rabbit IgG Antibody (Vector laboratories, Burlingame, CA, USA), for 15 min with Alexa Fluor ${ }^{\circledR} 680$ streptavidin and for 15 min with propidium iodide (both Invitrogen Life Technologies, Carlsbad, 
CA, USA). Finally the glass slides were imaged with a Leica TCS SPE confocal laser scanning fluorescence microscope (Leica DMRBE, Mannheim, Germany).

\section{Tail bleeding time}

Tail bleeding time measurements were performed as described earlier (15), with some modifications. Mice were anesthetized with an i.p. injection of ketamine $(100 \mathrm{mg} / \mathrm{kg})$ and xylazine $(20 \mathrm{mg} / \mathrm{kg})$. Mice were injected with either saline (control), $114 \mu \mathrm{g} \mathrm{UFH}$ or $570 \mu \mathrm{g} \mathrm{AADH}$. After 1 hour or 3 hours, the tip of the tail at $5 \mathrm{~mm}$ diameter was cut and immediately immersed in PBS pH 7.4 pre-warmed at $37^{\circ} \mathrm{C}$. Both the mice and prewarmed tubes with PBS were placed into an incubator at $37^{\circ} \mathrm{C}$ to maintain the mice at that temperature during the measurements. The total bleeding time was defined as the addition of bleeding periods during monitoring. Monitoring of the bleeding times was stopped at 10 minutes by cauterizing the tail to prevent excessive loss of blood.

\section{Liver viability assessment}

After CLP, as described above for the prophylactic regimen, animals were sacrificed 18 hours post ligation/puncture. The liver was harvested and sections prepared for TUNEL staining, allowing quantitation of alive/death cells in the organ.

\section{Acute lung injury model}

C57Bl/6 mice were treated with LPS $20 \mathrm{mg} / \mathrm{kg}$ i.p. and $570 \mu \mathrm{g}$ of AADH 1 hour after the LPS challenge. After 6 hours mice were euthanized for sample harvesting. 30 min before euthanasia, $5 \mu$ FITC-Ly-6G (Gr1) (eBioscience) and $100 \mu$ l Fluorescein isothiocyanate-Dextran (30 mg/ml FITC-Dextran; $70 \mathrm{kDa}$, Sigma-Aldrich) were applied by tail vein injection to label intravasal neutrophils and to assess plasma leakage, respectively. The trachea was dissected and cannulated (Portex FineBore Polythene Tubing, $0.28 \mathrm{~mm}$ inner diameter (ID)/0.61 mm outer diameter (OD), Smiths Medical International, Keene, NH). 5x $0.5 \mathrm{ml}$ PBS was injected and withdrawn. Thereafter, the ribcage was opened by a midline incision and the pulmonary vasculature was rinsed with $15 \mathrm{ml}$ ice-cold PBS with $0.5 \mathrm{mM}$ EDTA after cutting the inferior cava vein to facilitate exsanguination. The lungs were removed, minced and digested with liberase $(1: 20 ; 25$ mg Liberase RI/ml aqua, Roche Mannheim Germany). Digested lungs were passed through a cell strainer ( $70 \mu \mathrm{m}$; Miltenyi Biotec $\mathrm{GmbH}$, Bergisch Gladbach, Germany) and the resulting single cell suspension was centrifuged for $5 \mathrm{~min}$ at $300 \mathrm{~g}$. The pellets were resuspended in $1 \mathrm{ml}$ Hank's balanced salt solution with $0.3 \mathrm{mmol} / 1$ EDTA and $0.1 \%$ BSA. The bronchoalveolar lavage (BAL) fluid was centrifuged for 5 min at $300 \mathrm{~g}$. 


\section{Flow cytometry.}

Cell pellets were labeled with PerCP-Cy5.5 anti-mouse Ly-6G, PE anti-mouse CD115, APC-Cy7 anti-mouse CD45 and APC anti-Mouse F4/80 (all eBioscience). Neutrophils were identified by their typical appearance in the forward scatter-side scatter and as CD45 + CD115 - and PerCP- Gr1 + cells. Within the lung, FITC-Gr1 antibody was used to distinguish between interstitial neutrophils (CD45 +, CD115 -, PerCP- Gr1 +, FITC-Gr1 -) and intravasal neutrophils (CD45 +, CD115 -, PerCP- Gr1 +, FITC-Gr1 + ). All studies were performed on a FACS Canto II (Becton Dickinson, San Jose, CA) and data were analyzed using FlowJo software (Tree Star, Ashland, OR).

\section{Lung Permeability}

FITC-Dextran (70 kDa, Sigma-Aldrich) was used to assess vascular leakage. $100 \mu \mathrm{l}$ FITC-Dextran $(30 \mathrm{mg} / \mathrm{ml})$ were administered by tail vein injection $30 \mathrm{~min}$ prior to euthanasia and dye extravasation was used to assess change in vascular permeability. The fluorescence of $100 \mu \mathrm{l} \mathrm{BAL}$ supernatant (FluoBAL) and of $50 \mu \mathrm{l}$ serum (FluoSerum) was measured and clearance volume was expressed in microliter VPerm $=[$ (FluoBAL $/$ $100 \mu \mathrm{l}) * \mathrm{BAL}$ volume] / (FluoSerum / $50 \mu \mathrm{l})$.

\section{Lung histology}

After completion of the experiment, one part of the right lung was fixed in formalin, embedded in paraffin wax and stained with Mayer's haematoxylin and eosin for histological examination. We scored histological sections based on a scheme outlined in the table below which is compliance with recommendations from the American Thoracic Society outlining standardized guidelines for models of ALI in animals (16).

\begin{tabular}{lccc}
\hline & \multicolumn{3}{c}{ Score per field } \\
\cline { 2 - 4 } parameter & 0 & 1 & 2 \\
\hline A. alveolar neutrophils & none & $1-5$ & $>5$ \\
& & & \\
\hline B. interstitial neutrophils & none & $1-5$ & $>5$ \\
\hline $\begin{array}{l}\text { C. hyaline membranes } \\
\text { D. proteinaceous debris filling the }\end{array}$ & none & 1 & $>1$ \\
airspaces & & 1 & $>1$ \\
\hline \begin{tabular}{l} 
E. alveolar septal thickening \\
\hline
\end{tabular} & & & \\
\hline
\end{tabular}

Score $=[(20 x A)+(14 x B)+(7 x C)+(7 x D)+(2 x E)] /($ number of fields $x 100)$ 


\section{Protein concentration and cytokine measurements of the $B A L$}

The protein content of the BAL supernatants was assessed using the Bio-Rad Protein Assay based on the method of Bradford (Bio-Rad Laboratories Germany). Measurement of absorbance at $595 \mathrm{~nm}$ was performed with a microplate reader (infinite 200, Tecan Group Switzerland). TNF and IL6 in the BAL were assessed using a commercially available ELISA (R\&D Systems).

\section{Statistical Analyses}

Results are expressed as mean \pm SD. 1-Way ANOVA followed by Newman-Keuls post tests were used as appropriate to determine significance between groups or Dunnett post test in case of comparison to the control value. A P-value less than 0.05 was regarded as statistically significant, as indicated by *. Comparison of survival curves was done through calculation using the Log-rank (Mantel-Cox) and Gehan-BreslowWilcoxon test to calculate significance using GraphPad Prism 5.0 software (GraphPad Software, Inc.). Significance is indicated by **.

\section{Results and discussion}

Antithrombin affinity chromatography yielded a fraction of UFH, AADH, with strongly decreased anticoagulant activity (Figure 1A) as determined by CAT (12), which shows that AADH has retained $0.2 \%$ to $0.5 \%$ of the anticoagulant activity of UFH. Both UFH and AADH inhibited dose-dependently the cytotoxic activity of purified histones in cell culture (Figure 1C-D). To analyze whether AADH binds to histones, direct binding of $\mathrm{AADH}$ to histones was verified by surface plasmon resonance (Figure 1B). Binding affinity between histone $\mathrm{H} 3$ and $\mathrm{AADH}$ was characterized by an apparent dissociation constant of $86 \mathrm{nM}$. We further demonstrated in vitro, employing isolated human neutrophils, that addition of AADH to neutrophils that have produced NETs (5) (Figure 2A) results in colocalization of AADH with extracellular DNA fibers and histones (Figure 2B-C). Thus, both UFH and AADH possess cytoprotective properties, conferred by complexation of histones, because we verified that heparin-histone complexes are devoid of cytotoxic properties (Figure 3A).

To further test our hypothesis on the cytoprotective activities of AADH and translate our in vitro findings into an in vivo system, we next evaluated the effects of $\mathrm{AADH}$ on the overall mortality rate in a mouse model of fatal liver injury using ConA-triggered activation of $\mathrm{T}$ cells to mimic sterile inflammation (Figure $3 \mathrm{~B}$ ). It has been described that extracellular histones are the major mediators of death in this model (17). We measured plasma levels of histone $\mathrm{H} 3$ by semiquantitative western blotting (17) and showed that ConA injection increased circulating histone $\mathrm{H} 3$ levels in all animals (Figure 3C). In treated animals, appearance of histones was delayed and from our in vitro studies and 

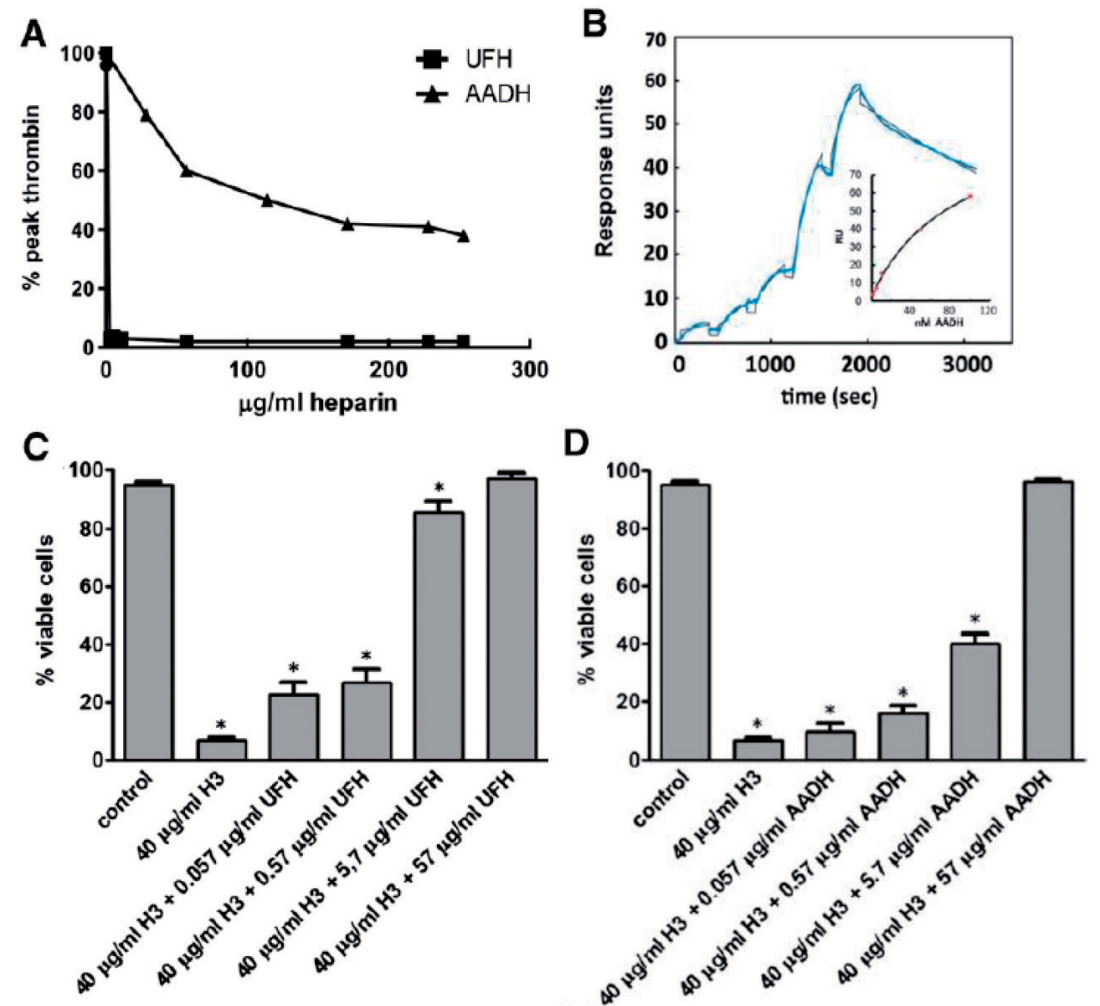

Figure 1. In vitro heparin characterization

A) In vitro effects of varying amounts of $\mathrm{UFH}$ and $\mathrm{AADH}$ on thrombin generation in normal pooled mouse plasma as measured by CAT (13). B) Surface plasmon resonance-analysis of AADH binding to immobilized histone $\mathrm{H} 3$ with collected data points in blue and data fit in black. Saturation of binding was verified by analysis of the binding data (insert). Variable amounts of UFH (C) or AADH (D) and histone H3 (H3) were added to EA.hy926 cells and cell viability was quantitated with flow cytometry. Each bar represents the average $6 \mathrm{SD}$ of at least 5 independent experiments. Statistical significance $(\mathrm{P}<0.05)$ was tested using one-way analysis of variance with Dunnett post hoc test. ${ }^{*}$ Indicates significant difference compared with control samples.

reported in vivo cytotoxicity of extracellular histones, we conclude that histones detected in the treated group are at least partly complexed to AADH. Injection of ConA caused significant differences in 24-hour survival between the AADH treated and control groups $(\mathrm{P}<0.0005)$ (Figure 3B), with only a moderate effect of AADH on thrombin formation (supplemental Figure 3D). We verified that the moderate anticoagulant effect seen is independent of any antithrombin-mediated activities of AADH and can be attributed to heparin cofactor II stimulation, the plasma concentration of which does not allow complete inhibition of thrombin formation (18). Together, our in vitro and in vivo results strongly indicate that $\mathrm{AADH}$ treatment results in complexation and 
inactivation of circulating histones with a concomitant improvement of survival, in agreement with the observation that $\mathrm{AADH}$ abolished cytotoxic effects of histones on endothelial cells by complexation (Figure 3A).
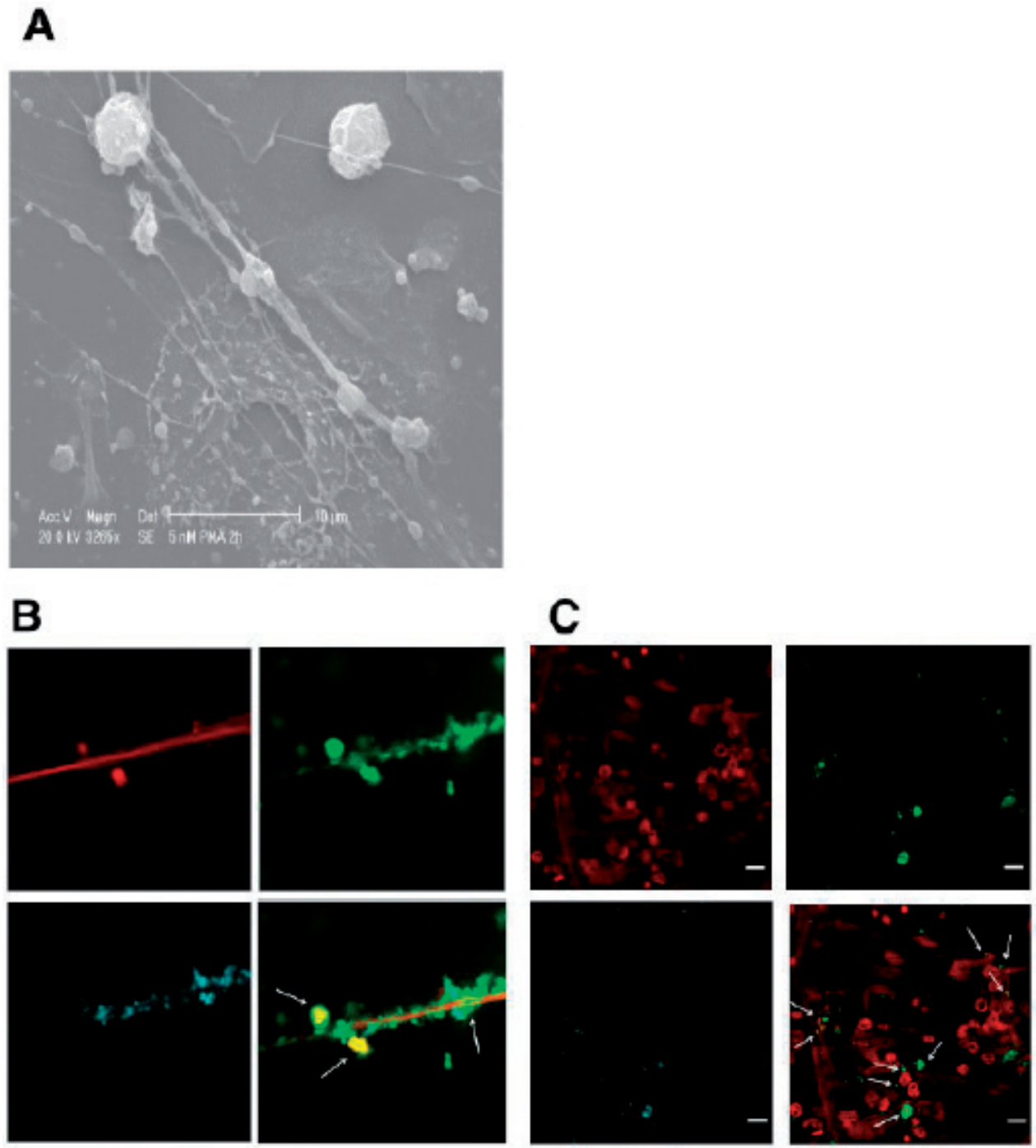

\section{Figure 2. In vitro visualization of NETs}

A) Scanning electron micrograph of phorbol myristate acetate (PMA) stimulated human neutrophils. B-C) Fluorimaging analysis of PMA-stimulated human neutrophils stained with propidium iodide (red), FITClabeled AADH (green), or anti-histone $\mathrm{H} 2 \mathrm{~B}$ antibody (blue). B) A detail showing colocalization (yellow, arrow) of histones, DNA, and AADH on extracellular DNA fibers. C) Representative presentation of a larger field with NETs, where colocalization (yellow) was observed for histones, DNA, and AADH. Arrows indicate several of the colocalization sites. Bar represents $20 \mathrm{~mm}$ for histology. 

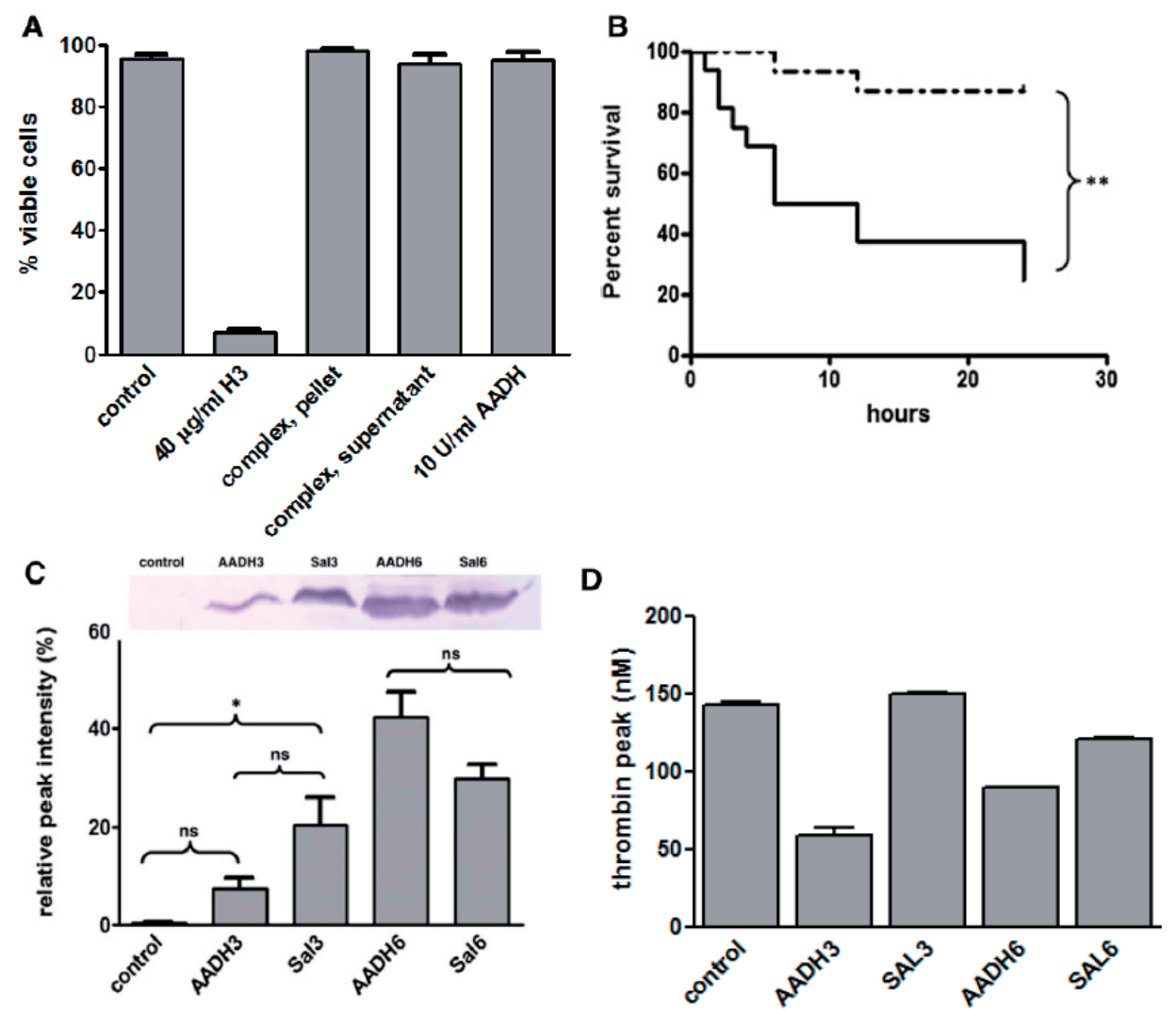

Figure 3.

A) Histones, complexed histones, supernatant of the precipitation reaction and only AADH are added to EA.hy926 cells and cell viability is quantitated with flow cytometry. As a control, cells to which control buffer was added were taken. B) Twenty-four-hour survival in mice challenged through intravenous injection of $30 \mathrm{mg} / \mathrm{kg}$ ConA following intraperitoneal injection of $570 \mu \mathrm{g}$ AADH ( $\mathrm{n}=15$, dashed line) or saline ( $\mathrm{n}=16$, solid line). Found differences are significant $(\mathrm{P}=0.0005$, with hazard ratio $=8.384$ and $95 \%$ confidence interval (CI) of ratio 2.371-21.79). C) Plasma of saline- or AADH-treated mice was collected 3 or 6 hours after ConA challenge and pooled $(n=6)$. Histone H3 content was characterized by Western blotting. For comparison, a pooled mouse plasma sample from mice not challenged with ConA is shown. Relative densities as determined by densitometry using ImageJ are indicated. D) From the same samples as in Figure 3C, thrombin generation was measured by CAT as described (13).

In contrast to UFH, AADH treatment of unchallenged mice with a dose 5 times that of UFH caused only moderate anticoagulation of blood plasma (Figure 4A) and no significant prolongation of tail bleeding time (Figure 4B). Furthermore, AADH treatment had no significant effects on plasma levels of the cytokines interleukin-6, interleukin-10, and tumor necrosis factor at 3 and 6 hours after ConA challenge (data not shown). 
To further investigate the potential therapeutic value of $\mathrm{AADH}$, we injected AADH in a mouse CLP model known to mimic human sepsis (19) and an LPS injection model that mimics TLR-mediated septic shock (19). As observed in the ConA model, AADH is able to significantly increase survival both when given prior to cecal puncture as well as when given 4 hours after puncture (Figure 4C) and when given 1 hour after LPS challenge (Figure 5A). Protection of organs is likely to contribute to overall survival as was concluded from a liver viability assessment after CLP (Figure 4D) and lung tissue histological analysis (Figure 5B-C). Administration of AADH appears to reduce neutrophil influx, protects against intrapulmonary protein leakage and capillary-alveolar leakage, and is accompanied by reduction of cytokine release (Figure 5D-G).

A

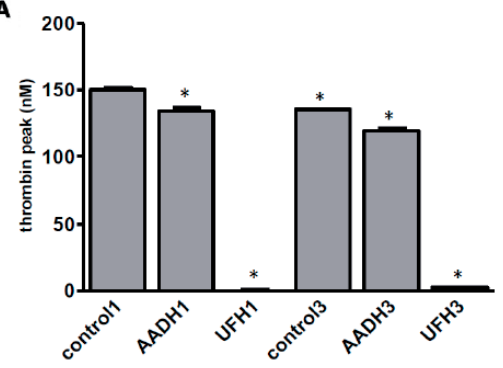

C

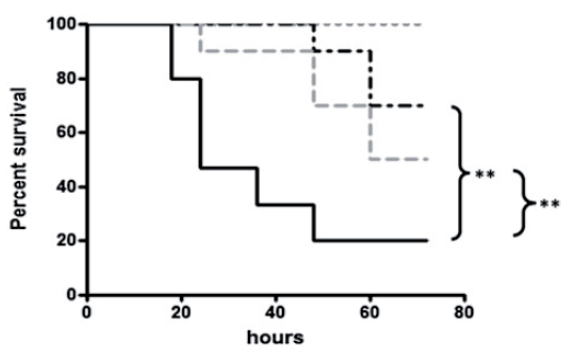

B
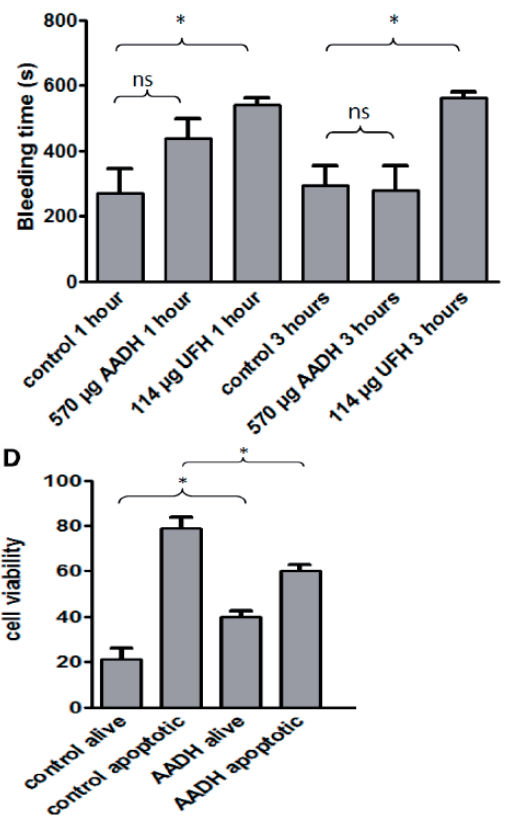

Figure 4.

A) Blood of saline, $570 \mu \mathrm{g}$ AADH or $114 \mu \mathrm{g}$ UFH-treated control mice (an equivalent of $20 \mathrm{U}$ UFH) was collected 1 or 3 hours after injection and equal plasma volumes were pooled $(n=6)$. Thrombin generation in platelet-poor plasma was measured by CAT as described. B) Tail bleeding times in mice as measured 1 and 3 hours after injection of respectively $114 \mu \mathrm{g}$ UFH or $570 \mu \mathrm{g}$ AADH. C) Seventy-two-hour survival in CLP-challenged C57Bl/ 6 mice treated by intraperitoneal injection with $570 \mu \mathrm{g}$ AADH 12 hours before, directly after, and 12 hours after CLP (prophylaxis, $n=10$, dashed black line) or only 4 hours after CLP $(\mathrm{n}=10$, dashed gray line). Saline-treated, CLP-challenged mice are shown as a solid black line $(\mathrm{n}=15)$, whereas survival of sham-treated animals is shown as a dotted gray line $(\mathrm{n}=15)$. Differences between AADH-treated and nontreated groups are significant (for both the prophylactic and treatment regimes, with $\mathrm{P}=0.0031$ and $\mathrm{P}=0.0275$, respectively, and hazard ratios $=5.471$ and $3.345,95 \% \mathrm{CI}$ of ratio 1.774 16.87 and 1.143-9.787, respectively. D) Quantitative analysis of liver viability as detailed in the methods section with timing and doses as described for the prophylactic regimen. Shown are the percentages of alive / apoptotic cells from liver sections for untreated $(n=8)$ or treated mice $(n=8)$. 

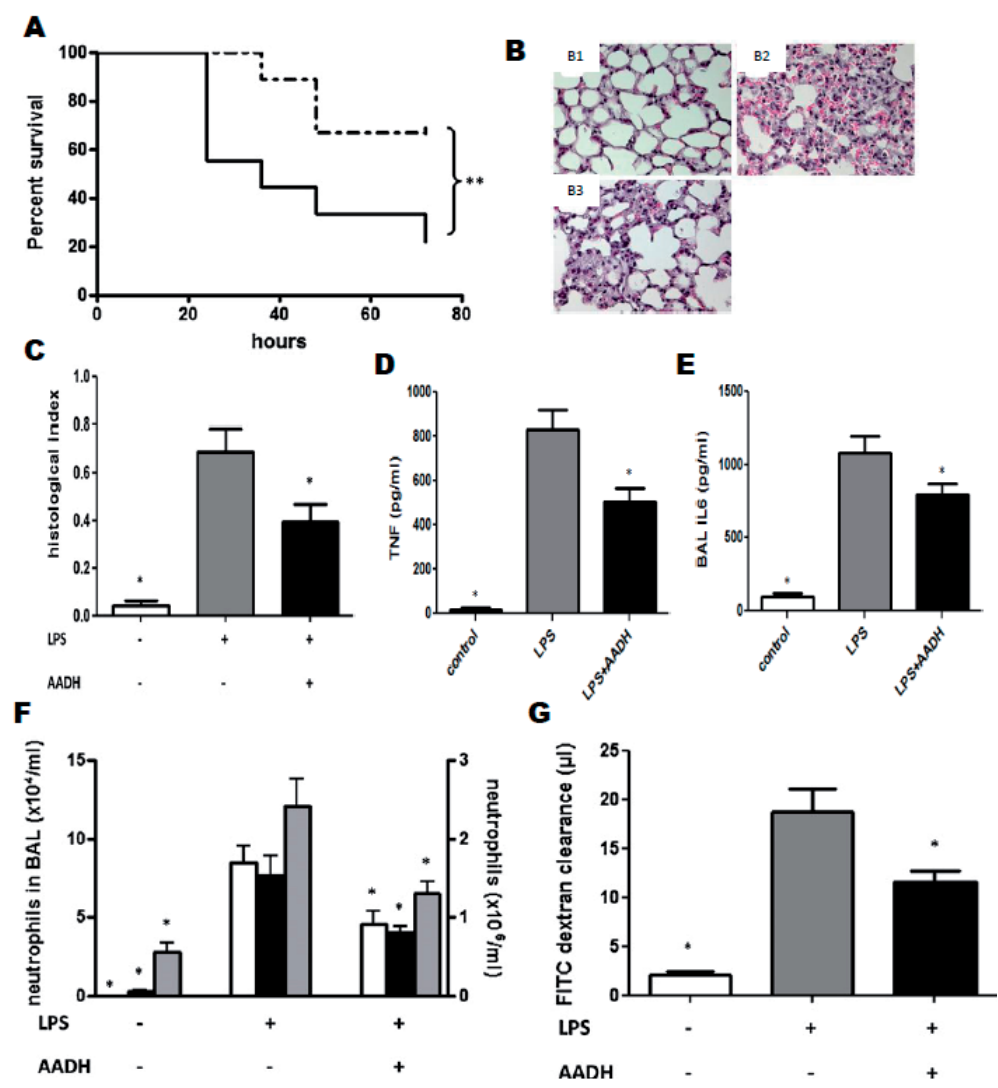

Figure 5. Effect of AADH in a mouse model of LPS-induced sepsis

C57Bl/ 6 mice were treated with LPS $20 \mathrm{mg} / \mathrm{kg}$ i.p. and $570 \mathrm{mg}$ AADH after 1 hour of the LPS challenge. Mice were euthanized 8 hours after LPS challenge. (A) Survival in mice receiving LPS and vehicle (solid line) and mice receiving LPS plus AADH (dashed line) ( $\mathrm{n}=9 /$ group). The difference between groups is significant, with $\mathrm{P}=0.0375$ and hazard ratio $=5.4328$ and $95 \% \mathrm{CI}$ of ratio 1.088-17.22. (B) Representative histological hematoxylin and eosin-stained sections of lungs from mice receiving saline (B1), LPS + vehicle control (B2), or LPS + AADH (B3). (C) Quantification of histological analysis, $\mathrm{n}=4$ per bar. Concentration of TNF (D) and IL6 (E) in the BAL as assessed by ELISA. (F) Quantification of neutrophils in the bronchoalveolar lavage (BAL) (white bars, left y-axis) of adherent, intravascular (black bars, right y-axis), and interstitial (gray bars, right y-axis) neutrophils. Discrimination between neutrophils in either location was made based on an antibody to Ly6G injected 5 minutes prior to euthanasia ( $\mathrm{n}=6-8$ for each bar). $(\mathrm{G})$ Assessment of lung plasma leakage based on the exudation of the plasma tracer FITC dextran ( $\mathrm{n}=6-8)$. Statistical significance was tested using one-way analysis of variance with Dunnett post hoc test. *Indicates significant difference $(\mathrm{P}<0.05)$ compared with the LPS-challenged group. 
We conclude that AADH, a low anticoagulant fraction of UFH, has therapeutic potential to treat sepsis and other hyperinflammatory conditions in which excessive neutrophil activation with release of histones is part of the host response. Given the huge clinical experience with UFH and the anticipated safe profile of AADH, we expect low thresholds for commencing clinical studies in the near future.

\section{Acknowledgments}

This work was supported by the Netherlands Organization for Scientific Research (medium investment grant to G.A.F.N.) and the Cardiovascular Research Institute Maastricht (to G.A.F.N.). O.S. is supported by the Netherlands Organization for Scientific Research (VIDI project 91712303) and the Deutsche Forschungsgemeinschaft (SO876/3-1, SO876/6-1, FOR809, SFB914-B08). 


\section{References}

1. Wiersinga WJ. Current insights in sepsis: from pathogenesis to new treatment targets. Curr Opin Crit Care. 2011;17(5):480-486.

2. Brinkmann V, Reichard U, Goosmann C, et al. Neutrophil extracellular traps kill bacteria. Science. 2004;303(5663):1532-1535.

3. Clark SR, Ma AC, Tavener SA, et al. Platelet TLR4 activates neutrophil extracellular traps to ensnare bacteria in septic blood. Nat Med. 2007;13(4):463-469.

4. Xu J, Zhang X, Pelayo R, et al. Extracellular histones are major mediators of death in sepsis. Nat Med. 2009;15(11):1318-1321.

5. Saffarzadeh M, Juenemann C, Queisser MA, et al. Neutrophil extracellular traps directly induce epithelial and endothelial cell death: a predominant role of histones. PLoS ONE. 2012;7(2):e32366.

6. Chaput C, Zychlinsky A. Sepsis: the dark side of histones. Nat Med. 2009;15(11):1245-1246.

7. Jaimes F, De La Rosa G, Morales C, et al. Unfractioned heparin for treatment of sepsis: a randomized clinical trial (The HETRASE Study). Crit Care Med. 2009;37(4):1185-1196.

8. Dhainaut JF, Shorr AF, Macias WL, et al. Dynamic evolution of coagulopathy in the first day of severe sepsis: relationship with mortality and organ failure. Crit Care Med. 2005;33(2):341-348.

9. Cox MM, Nelson DL. Lehninger, Principles of Biochemistry. 5th ed. W.H. Freeman \& Company; 2008.

10. Kent PW, Hichens M, Ward PF. Displacement fractionation of deoxyribonucleoproteins by heparin and dextran sulphate. Biochem J. 1958;68(4):568-572.

11. Demidenko O, Tsvetkova S. Action of heparin on protein fractions of isolated nuclei and on their DNA content. Histochemistry. 1978;57(3): 265-271.

12. Hemker HC, Giesen P, Al Dieri R, et al. Calibrated automated thrombin generation measurement in clotting plasma. Pathophysiol Haemost Thromb. 2003;33(1):4-15.

13. Tchaikovski SN, VAN Vlijmen BJ, Rosing J, Tans G. Development of a calibrated automated thrombography based thrombin generation test in mouse plasma. J Thromb Haemost. 2007;5(10):20792086.

14. Pluder F, Barjaktarovic Z, Azimzadeh O, et al. Low-dose irradiation causes rapid alterations to the proteome of the human endothelial cell line EA.hy926. Radiat Environ Biophys. 2011;50(1):155-166.

15. Akbar H, Shang X, Perveen R, et al. Gene targeting implicates Cdc42 GTPase in GPVI and non-GPVI mediated platelet filopodia formation, secretion and aggregation. PLoS ONE. 2011;6(7):e22117.

16. Matute-Bello G, Downey G, et al; Acute Lung Injury in Animals Study Group. An official American Thoracic Society workshop report:features and measurements of experimental acute lung injury in animals. Am J Respir Cell Mol Biol. 2011;44(5):725-738.

17. Xu J, Zhang X, Monestier M, Esmon NL, Esmon CT. Extracellular histones are mediators of death through TLR2 and TLR4 in mouse fatal liver injury. J Immunol. 2011;187(5):2626-2631.

18. Briginshaw GF, Shanberge JN. Identification of two distinct heparin cofactors in human plasma. II. Inhibition of thrombin and activated factor X. Thromb Res. 1974;4(3):463-477.

19. Grommes J, Alard JE, Drechsler M, et al. Disruption of platelet-derived chemokine heteromers prevents neutrophil extravasation in acute lung injury. Am J Respir Crit Care Med. 2012;185(6):628636. 



\section{Chapter 8}

Summary and General Discussion 
Chapter 1 introduces the subject of this thesis. An overview of the extrinsic and intrinsic coagulation pathways is given, followed by a detailed description of the anticoagulants activated protein C (APC) and heparin. Next to their anticoagulant functions, these biomolecules possess cytoprotective activities via anti-inflammatory, anti-apoptotic, endothelial barrier stabilizing and histone neutralizing properties. To improve the in vivo efficacy of APC and heparin, engineered variants with strongly diminished anticoagulant properties were designed. The cytoprotective effects of these variants were tested in murine disease models of atherosclerosis, myocardial ischemia/reperfusion injury and sepsis.

\section{Structure-function relationship of APC}

APC is an anticoagulant serine protease that controls thrombin formation via proteolysis of active coagulation factors V (FVa) and VIII (FVIIIa). Proteolysis of FVa and FVIIIa is most efficient in the presence of phospholipids and cofactors protein $\mathrm{S}$ and FV (Figure 1A, chapter 2). The protective effects of APC administration in animal models of sepsis, stroke, myocardial infarction and lung injury can however not be explained by the anticoagulant effects of APC, but rather by its cytoprotective effects that are dependent on the presence of the protease activated receptor 1 (PAR-1) and the endothelial protein $\mathrm{C}$ receptor (EPCR). Recently, it was shown that cell-receptors like Tie2, S1P1, CD11b/CD18, ApoER2 (LRP8) and epidermal growth factor (EGF) receptor are also involved in APC signaling. APC can protect cells by its endothelial barrier stabilizing, anti-inflammatory and anti-apoptotic functions. These cytoprotective effects and the molecular mechanisms identified so far are summarized in Figure 1B-D of chapter 2.

Chapter 2 presents an extensive overview of the current state of the art on the structure of APC, its dual anticoagulant and cytoprotective functions and the effects of natural and engineered mutations of APC on its functions. Structural bioinformatics analyses allowed the identification of the mosaic domain structure of APC, containing an $\mathrm{N}$-terminal $\gamma$-carboxyglutamic acid (Gla)-domain, two EGF-like domains, a linking peptide and a C-terminal trypsin-like serine protease domain which harbors the active site triad composed of the residues D257, H211 and S360. Studies describing the effects of both natural and engineered APC mutations on its function were analyzed and used to map the various APC functions and interactions onto its 3D structure (Figure 6, chapter 2). The active site triad in the serine protease domain is indispensable for proteolysis of FVa and FVIIIa. Additionally, positively charged exosites on the APC surface, including the 191-loop (residues 190-193), the $\mathrm{Ca}^{2+}$-binding loop (residues 225-235) and the autolysis loop (residues 302-317) are important for interaction with FVa cleavage sites. Furthermore, interactions of APC with phospholipids, EPCR and protein $\mathrm{S}$ are essential for the anticoagulant function of APC. For binding of APC to phospholipid membranes and EPCR, the $\mathrm{Ca}^{2+}$-dependent conformation of the 
Gla-domain as well as several individual residues in the Gla-domain are essential. This information has provided a rational basis for protein engineering of APC variants with improved membrane and EPCR binding capacities that show increased anticoagulation and are therefore more effective for the pharmacological use in thrombosis models (13). For interaction with its cofactor protein $\mathrm{S}$. the autolysis loop of APC and residues in the Gla-domain (residue L38), EGF domain (residue D71) and linking peptide (residue E149) are important.

Mutagenesis studies have indicated that the more recently discovered cytoprotective functions of APC can be linked to residue E149 located in the EGF domain and residues E330, E333 and S360 located in the serine protease domain. The molecular mechanism which reconciles the influence of E149 on cytoprotection is not known yet. E330 and E333 are essential for interaction with PAR-1 and S360 is part of the catalytic triad and therefore needed for proteolysis of PAR-1. However, we have shown in this thesis that even in the absence of residue S360, APC can provide cytoprotection in an in vivo model of acute myocardial I/ $\mathrm{R}$ damage.

The effects of a number of APC variants, which have been characterized for their anticoagulant properties, have not yet been investigated in the context of their cytoprotective properties, so potentially more residues involved in cytoprotection will be identified in the future. This will aid in the engineering of an APC variant that is devoid of anticoagulant function, but has increased cytoprotective properties and can therefore provide optimal cell-protection in a number of relevant clinical conditions without causing bleeding complications.

\section{Identification of novel small molecule inhibitors of APC}

Several in vitro and in vivo observations suggest that APC resistance and a decreased activity of APC are associated with a relative shift in the haemostatic balance towards a more procoagulant state. Hemophilia A patients who have co-inherited the FV Leiden mutation, resulting in APC resistance, have a delayed onset of symptoms after birth and a decreased bleeding frequency and factor concentrate utilization $(4,5)$. We show that $e x$ vivo FVa activity in human plasma is stable when its proteolytic downregulator APC is inhibited, which may lead to increased in vitro thrombin generation. So, possibly therapy based on the inhibition of APC has potential to prolong the in vivo lifetime of FVa and give rise to increased thrombin generation in individuals with bleeding tendencies, such as hemophilia A patients. In chapter 3 we used a validated in silico structurebased virtual screening approach to rationally discover small drug-like molecules that inhibit the interaction of a predominant exosite on the surface of the serine protease domain of APC with its substrate FVa. By targeting this exosite and leaving the catalytic site unaffected, we hypothesize that the compounds will only affect the anticoagulant properties of APC and will not influence its cytoprotective functions. After in silico screening and prioritizing, the predicted best 624 compounds were selected and 
purchased for in vitro assessment of their properties in a functional assay that quantitates their capacity to inhibit the inactivation of FVa by APC. At a concentration of $100 \mu \mathrm{M}$, the selected small molecules did not show complete inhibition of FVa inactivation, which most likely reflects the relatively extended surface that is involved in the interaction between APC and FVa. The top 20 functionally active compounds were further tested for their direct binding ability to APC via surface plasmon resonance (SPR). Kd values of the best 15 compounds were in the range of $10^{-3}-10^{-5} \mathrm{M}$. To check whether the compounds were directed at the targeted exosite, two different APC variants containing mutations in the targeted exosite were constructed and binding of the compounds to these APC variants was investigated. Five compounds showed a $\sim 100-500$-fold decreased affinity for the APC variants compared to wt-APC. This loss of affinity can be explained by the reduction of molecular interactions that could be formed between the mutated exosites and the compounds. Four compounds showed 10-fold increased affinity for at least one of the APC variants. Most likely this is explained by the fact that these compounds become (positively) charged upon solvation and therefore bind better when the big aromatic ring containing tyrosine residue or the positively charged lysine/ arginine residues present in wt-APC are replaced by alanine residues.

Finally we studied potential preference of the compounds for APC-catalyzed cleavage at either R506 or R306, since it was described that the targeted site is particularly important for proteolysis at R506 $(6,7)$. 60\% of compounds had no preference for either of the cleavage sites, while $20 \%$ of compounds showed preferred cleavage at either R306 or R506. These findings are in line with earlier studies concluding that the APC exosite involved in interaction with FVa is large and that the epitopes important for cleavage at R306 or R506 do not fully overlap (6-10). Of note, the compounds specific for R506 scored on average better in the functional FVa inactivation assay than the compounds specific for R306. This appears in contradiction to an earlier study that described that R306 is the most important APC-cleavage site for FVa inactivation in vitro (11), but may merely illustrate the fact that the functional assay used is more sensitive to functional changes due to cleavage at R506 than to cleavage at R306.

Interestingly, there was little correlation between the functional activity of the best 20 compounds and their direct binding affinity to APC. This implies that tight binding per $s e$ is not sufficient to prevent the interaction between APC and FVa, but that the exact position where the compound binds on the relatively large exosite surface plays an important role. Thus, when the goal of a virtual ligand screening campaign is to identify compounds that interfere with the function of a protein, it is recommended to include a functional screening assay at an early stage of the discovery pipeline and not only to measure binding affinity. In our case the functional assay measurements in the absence of APC yielded additional information on the potential binding of compounds to offtargets like the homologous serine proteases FXa and thrombin and therefore we could exclude non-specific compounds from further study already after an initial screening. 
Previous studies (11-14) have already identified peptidic and peptide-like compounds, RNA aptamers and ssDNA aptamers that inhibit the proteolytic activity of APC with reasonably good specificity. The disadvantage of those types of inhibitors is that they inhibit the amidolytic activity of APC and therefore possibly affect the cytoprotective functions of APC. A further drawback of the peptide-like compound (13) is that it contains a hydrophilic benzamidine moiety that possibly can raise hepatotoxicity. The inhibitors identified by others show higher affinity for APC than the small molecules we identified, but the big advantage of the use of low molecular weight compounds is that they have better in vivo bioavailability and metabolic stability and have the potential to evolve into orally administered molecules (15).

This study shows that small molecules identified via in silico virtual ligand screening and selected through application of in vitro functional and direct binding assays, can inhibit protein-protein interaction between APC and FVa. The identified small molecules should be further optimized and developed to increase their affinity and functional activity. Subsequently they can potentially be used to increase in vivo thrombin generation by specific inhibition of the anticoagulant functions of APC in individuals with bleeding tendencies, like hemophilia A patients.

\section{Effects of exogenous APC in murine myocardial I/R injury and atherosclerosis}

We hypothesized that the anti-inflammatory properties of APC (summarized in Figure $1 \mathrm{~B}$, chapter 2) can influence the development of acute and chronic inflammatory diseases. In chapter 4 we investigated the effects of exogenous human (h) wt- and hS360A-(A)PC administration in murine models of acute myocardial I/R injury and the chronic inflammatory disease atherosclerosis.

First, the effects of exogenous hAPC administration on infarct area and IL-6 levels were examined in a murine myocardial I/R model. Mice received $0.4 \mathrm{mg} / \mathrm{kg}$ hwtPC, hS360A-PC, hwt-APC, hS360A-APC or placebo (saline) both 15 minutes after induction of ischemia and 5 minutes after induction of reperfusion. We show that hwt, hPC, hS360A-PC, hwt-APC and hS360A-APC all significantly reduced the myocardial infarct area. This finding can be explained by the fact that binding of either wt-(A)PC or S360A-(A)PC to EPCR, leads to dissociation of EPCR from caveolin-1 in lipid rafts. In this condition, PAR-1 cleavage by either thrombin or APC results in anti-inflammatory PAR-1 signaling via $\beta$-arrestin- 2 and Gi/o G-proteins and subsequent activation of Rac1 and inhibition of NFKB signaling (16-19). Next to the aforementioned mechanism, (A) PC can interact via its Gla-domain with phospholipids incorporated in LDL particles, resulting in decreased ROS formation and lipid peroxidation. Additionally, the protective effect of zymogen PC can potentially involve in vivo activation to APC. The advantage of S360A-(A)PC administration over wt-(A)PC administration in a clinical therapeutic context is that this variant will not induce bleeding complications and will have a longer in vivo half life, because it cannot be inhibited by circulating serpins (20). 
S360A-(A)PC has some residual anticoagulant activity, but we believe that this did not contribute to the reduced myocardial infarct area observed in S360A-(A)PC treated mice. In vivo thrombin inhibition by heparin or dansyl glutamyl-glycyl-arginyl chloromethyl ketone-treated activated factor X (DEGR-FXa) failed to reduce I/R injury $(21,22)$. Wang and coworkers (23) showed in a murine focal ischemic stroke model that the cytoprotective-selective 5A-mutant (that has much reduced anticoagulant properties) decreased infarct volume and improved neurological outcome, while an anticoagulantselective E149A-APC variant worsened neurological outcome, increased infarct volume and increased bleeding risk.

Contradictory to the results of Loubele and coworkers (22) we did not observe an effect of $\mathrm{h}(\mathrm{A}) \mathrm{PC}$ administration on the lowering of IL-6 levels in heart homogenates after acute myocardial I/R injury. This can partially be explained by the 5 -fold lower IL- 6 levels that we observed in placebo treated mice, leaving less room for a further decrease by $\mathrm{h}(\mathrm{A}) \mathrm{PC}$ treatment. The fact that we used h(A)PC and the study by Loubele et al. (22) murine (m)APC may also have contributed to the observed discrepancy. Both hAPC and $\mathrm{mAPC}$ have equal in vitro proteolytic activities, but $\mathrm{mAPC}$ has a higher affinity for mPAR-1 and is more potent in murine stroke models than hAPC, probably due to its different sequence and post-translational modifications $(24,25,26)$.

Having observed that both hwt- and hS360A-(A)PC provide protection in the acute inflammatory myocardial I/R model, we subsequently investigated the effect of 8 weeks twice weekly administration of $0.2 \mathrm{mg} / \mathrm{kg}$ hwt-PC, hS360A-PC, hwt-APC, hS360A$\mathrm{APC}$ or placebo (saline) in a murine $\mathrm{ApoE}^{-/-}$model of the chronic inflammatory disease atherosclerosis where we studied plaque development. Surprisingly, in none of the treatment groups the development of atherosclerotic plaques was significantly altered by hwt- or hS360A-(A)PC administration. In both hS360A-APC and hwt-PC treated mice, the collagen content of the plaques was significantly decreased. Moreover, in hS360A-APC treated mice, increased infiltration of leukocytes and T-lymphocytes into the plaques was observed. None of the treatments changed the innate and adaptive immune system as assessed by analysis of immune cells isolated from blood and spleen. Mice that had received hS360A-PC and hwt-APC had significantly decreased plasma IL-6 levels, which were in hS360A-PC treated mice accompanied by increased MCP-1 levels. Mice in all treatment groups had slightly but significantly increased cholesterol levels.

The inability of $\mathrm{h}(\mathrm{A}) \mathrm{PC}$ to influence plaque development can possibly be explained from its limited bioavailability. Previous research $(27,28)$ demonstrated that after i.p. administration of APC, its concentration in circulation was increased above baseline for 3 hours with a maximum reached after 20 min. Apparently the transient rises in concentration that were achieved twice weekly in our study, were not sufficient to initiate protective anti-inflammatory signaling that lasted long enough to provide long term protection against atherosclerosis during the time span of our study. To increase 
the efficacy of APC in future chronic disease studies it is recommendable to use a slowrelease device to administer APC, but this will require thermal stability of APC over time at body temperature. When this is not feasible, intravenous and more frequent APC injections will be the best alternative to improve the in vivo bioavailability and potency of APC.

In conclusion, we showed that both hS360A- and hwt-(A)PC reduce acute myocardial $\mathrm{I} / \mathrm{R}$ injury and have the potential to influence development of chronic inflammation as occurring during atherosclerosis.

\section{Effect of APC cleavage of histones on their cytotoxic properties}

It was recently discovered that APC has, next to its anticoagulant and cytoprotective effects, the ability to cleave extracellular histones (29). In chapter 5 we first explored the effect of calcium and phospholipids, known effectors of in vitro APC activity, on the proteolytic activity of wt- and 5A-APC towards histones and we next investigated the cytotoxicity of APC-cleaved histones towards endothelial cells. 5A-APC has, due to mutation of five positively charged amino-acids, located in an APC exosite important for electrostatic interaction with $\mathrm{FVa}$, a reported minimal anticoagulant activity but normal cytoprotective activity (30).

During a time-course of APC-catalyzed histone inactivation, proteolysis of histone $\mathrm{H} 3$ by either wt-APC or 5A-APC yields different cleavage products over time. Finally, end products of $\sim 12.3 \mathrm{kDa}$ and $\sim 9.7 \mathrm{kDa}$ are observed. The molecular weight (MW) of full length histone $\mathrm{H} 3$ is $15.3 \mathrm{kDa}$, so next to the observed end products two smaller fragments of $\sim 3 \mathrm{kDa}$ and $\sim 5.6 \mathrm{kDa}$ must have arisen simultaneously. The antibody used for Western blotting is raised against an epitope at the C-terminus of histone $\mathrm{H} 3$, so therefore these $\mathrm{N}$-terminal cleavage products are not visible on Western Blot. 5A-APC cleaves histone $\mathrm{H} 3$ with significantly higher efficiency than wt-APC. Neutralization of the positive charge in the exosite important for FVa binding probably increased the interaction with the highly positively charged histones and thereby resulted in more efficient binding and proteolysis.

Calcium ions are important for maintenance of the $\mathrm{Ca}^{2+}$-dependent conformation of APC and for interaction of APC with phospholipids. Therefore, the presence of calcium is indispensible for proper anticoagulant functioning of APC (31). We observed that optimal proteolysis of histone $\mathrm{H} 3$ occurs at $\mathrm{Ca}^{2+}$ concentrations of 1 and $2.5 \mathrm{mM}$. This is in line with the optimum of $\sim 2 \mathrm{mM}$ found for proteolysis of FVa by APC (32). These concentrations are very similar to the physiological range in plasma for total calcium of 2.10-2.55 mM and for ionized calcium of 1.15-1.30 mM (33).

Disruption of cell walls is a mechanism used by histones to facilitate killing of parasites and bacteria (34-36). Histones are capable of binding to phospholipid membranes of eukaryotic cells, they can transverse multilamellar and large unilamellar vesicle lipid bilayers and induce channel formation in plasma membranes $(37,38)$. 
The ability of histones to form channels is enhanced by the presence of negatively charged phospholipids, possibly because increased electrostatic interactions result in enhanced histone binding or increased stability of the bound histones to the membrane (38). In the present study we used small purified synthetic unilamellar phospholipid vesicles and have shown that these vesicles interacted with histones and reduced their cytotoxicity towards endothelial cells. In the presence of APC, phospholipids enhance the cleavage rate of histones by APC. Both in the absence and presence of APC, maximum protective effects were reached when vesicles contained PS and PE, next to the neutral phospholipid PC. Histones have a positive electrical charge and it is shown that they bind with high avidity to the anionic phospholipid PS (39). It has prior been demonstrated that vesicles containing a mix of PS and PC were optimal for proteolysis of FVa by APC $(32,40,41)$. However, two research groups $(42,43)$ showed that PE has added value for optimal anticoagulant activity of APC.

Next, we have investigated the effect of histone H3 cleavage on histone-mediated cytotoxicity and found that the cleavage products of $\sim 12.3 \mathrm{kDa}$ and $\sim 9.7 \mathrm{kDa}$ are not cytotoxic towards endothelial cells. Thus, histone cleavage by APC annihilates its detrimental effects towards cells. Finally we developed an algorhythm to predict at which positions APC can proteolyse its substrates and applied this to histone H3. For the prediction of possible cleavage sites in histone $\mathrm{H} 3$, information about cleavage sites of APC in different substrates and the expected MW of the histone $\mathrm{H} 3$ fragments estimated by Western blotting were implemented. To verify the results of the algorhythm we determined two cleavage sites of APC via $\mathrm{N}$ terminal sequencing. We found that the cleavage products of $\sim 12.3 \mathrm{kDa}$ and $\sim 9.7 \mathrm{kDa}$ originated from cleavages C-terminal of R26 and C-terminal of R52. These cleavage sites were correctly identified by our algorhythm, that further predicted R8/K9, R18, R128 and R131 as potential cleavage sites matching the MW of the other histone cleavage fragments. The R26 cleavage site had prior to our study already been identified in murine histone H3 (29), but the R52 cleavage site has been newly discovered by our research group.

The end products of histone $\mathrm{H} 3$ cleavage by APC did not possess cytotoxic properties towards endothelial cells. This explains why APC can potentially be used as a therapy for pathologic conditions accompanied by increased extracellular histone and nucleosome levels and supports earlier studies $(29,44)$ that have shown a survival benefit and histone $\mathrm{H} 3$ cleavage in APC treated baboons that had been subjected to a septic trigger. We hypothesize that $5 \mathrm{~A}$-APC administration will likewise be beneficial for this purpose, because this variant proteolyses histones more efficiently than wt-APC, is safe for administration in patients with a bleeding risk and its dose in other patients can safely be raised to increase the therapeutic potential even further. 


\section{Extracellular histones are associated with mortality and organ failure in sepsis}

In patients with systemic inflammation and sepsis, increased concentrations of circulatory nucleosomes were found $(45,46,47)$ and it was shown that traumatic injury patients had increased levels of circulating histones (48). In chapter 6 we measured extracellular histone $\mathrm{H} 3$ levels in plasma samples of 43 sepsis patients withdrawn within 24 hours after ICU admittance. We explored possible correlations of these plasma histone levels with disease severity, mortality and coagulation- and tissue-homeostasis parameters.

We found that 6 sepsis patients presented with high plasma histone $\mathrm{H} 3$ levels ranging from $22-126 \mu \mathrm{g} / \mathrm{ml}$, while all other patients had histone H3 levels below $10 \mu \mathrm{g} / \mathrm{ml}$. Extracellular histone H3 levels were comparable in sepsis, severe sepsis and septic shock patients. In non-survivors the concentration of circulating histones in plasma was significantly higher than in survivors of sepsis ( $\mathrm{P}=0.038$, Figure 1, Chapter 6). Extracellular histone $\mathrm{H} 3$ levels showed a significant positive correlation with sepsisinduced organ failure as assessed by the SOFA score $(\mathrm{P}=0.023)$, lactate dehydrogenase $(\mathrm{LDH})$ activity $(\mathrm{P}=0.000)$ and lactate levels $(\mathrm{P}=0.011)$. Significant negative correlations were observed between histone $\mathrm{H} 3$ levels and both platelet count $(\mathrm{P}=0.013)$ and antithrombin levels $(\mathrm{P}=0.025)$.

Extracellular histones can induce cell death via three different mechanisms. Firstly, they can directly induce cell death by interaction with and transversion over phospholipid membranes and formation of membrane pores $(37,38)$. Secondly, extracellular histones can induce MyD88 signaling via TLR2 and TLR4. This results in up-regulation of NF-KB and MAPK target gene expression and enhancement of cytokine production, leukocyte recruitment, inflammation, endothelial cell death and vascular permeability. Furthermore, extracellular histones can affect platelet function in a TLR2- and TLR4dependent way, resulting in activation of ERK, P38, AKT and NF-KB signaling pathways. Activation of platelets increases thrombin generation and induces platelet aggregation and clotting, resulting in rapid and profound thrombocytopenia (49-51). Finally, circulating histones can induce intracellular oxidative stress and consequently activate the NLRP3 inflammasome, a cytosolic platform that controls host responses to infection and injury, via caspase-1-dependent IL-1 $\beta$ and IL-18 release (52, 53). Together these mechanisms contribute to tissue injury and death in the context of disseminated intravascular coagulation (DIC) and sepsis (29, 54-56).

In both septic shock patients and non-survivors of sepsis a hypocoagulable state, characterized by increased PT and CAT lag times, decreased prothrombin/antithrombin levels and reduced platelet count, was observed. Parameters reflecting tissue homeostasis (LDH activity and lactate) were increased in non-survivors and septic shock patients. The presence of a hypocoagulable state in most severely ill patients is in agreement with results of thrombin generation experiments in sepsis patients in literature (5760). The observed reduction in platelet count and coagulation factors and prolonged 
clotting times in septic shock patients confirm previous observations $(57,60)$. Before development of a hypocoagulable state, the coagulation system of sepsis patients is typically systemically over-activated and DIC frequently occurs, causing consumption of platelets and pro- and anticoagulant factors (61). At the same time, concentrations of anticoagulant factors can drop due to decreased hepatic synthesis during the acute phase response and due to degradation by elastases released from activated neutrophils (62). Activated neutrophils can trigger NET formation and thereby form, in addition to dying cells, a source of extracellular histones. Increased lactate and LDH activity in sepsis patients can possibly originate from hypoxia and subsequent necrosis in organs due to deposition of fibrin and (micro)thrombi in afferent blood vessels (61). Alternatively a combination of increased aerobic glycolysis and decreased clearance of lactate by the liver during the acute phase response can cause elevation of $\mathrm{LDH}$ activity and lactate levels $(63,64)$. Increased lactate levels or inability of the body to clear lactate/LDH were identified as strong predictors of mortality in sepsis patients during the first 24-48 hours after ICU admittance (65-67), in agreement with the significantly increased LDH activity levels that we observed in non-survivors $(\mathrm{P}=0.001)$, compared to survivors of sepsis.

The development and progression of sepsis is a multi-factorial process. We proof that there is a strong correlation between extracellular histone levels and parameters reflecting coagulation- and tissue-homeostasis. All reflect ongoing systemic inflammation, coagulation, and organ failure and these parameters can potentially be used as biomarkers for prediction of disease severity and mortality in sepsis patients.

\section{Effect of non-anticoagulant heparin on histone-mediated cytotoxicity}

We hypothesized that heparin, due to its negative charge, could be a good candidate to bind to positively charged histones and neutralize their detrimental effects. The drawback of heparin for in vivo use is its associated risk for bleeding complications. Therefore we developed a non-anticoagulant antithrombin affinity-depleted heparin $(\mathrm{AADH})$ and investigated its in vitro and in vivo properties in chapter 7.

We found that $\mathrm{AADH}$ possessed only $0.2 \%$ to $0.5 \%$ of the anticoagulant activity of UFH in a thrombin generation assay, but inhibited the cytotoxicity of purified histones towards endothelial cells dose-dependently and to the same extent as UFH. We have shown via surface plasmon resonance that $\mathrm{AADH}$ binds to single histones with an affinity characterized by a dissociation constant of $86 \mathrm{nM}$ and that AADH co-localizes with NET-associated extracellular histones.

Next, we investigated the cytoprotective properties of $\mathrm{AADH}$ in three different in vivo murine models: a model of ConA-induced sterile inflammation wherein histones are the major mediators of death (54), in a CLP model that mimics human sepsis (68) and in a LPS model mimicking TLR-mediated septic shock (68). We confirmed that ConA-injection increased plasma levels of extracellular histones in mice. Concomitant 
injection of AADH delayed the appearance of histones in plasma. Surprisingly, six hours after ConA-challenge histone levels in both the AADH-treated and control group were comparable, while the treated group showed a clear survival benefit (Figure 3B, chapter 7). To explain this apparent paradoxal appearance of extracellular histones, we hypothesize that the histones detected in the AADH-treated group were at least partly complexed with AADH. Via Western blotting of mouse plasma samples under non-denaturating conditions we found indications that this might indeed be the most plausible explanation. We showed that complexed histones do not possess cytotoxicity towards endothelial cells in vitro. AADH treatment significantly increased survival of mice in all three murine sepsis models compared to mice in the control groups. Even when AADH was administered 4 hours after induction of sepsis, mimicking a treatment regimen, survival was significantly improved. The beneficial effects of $\mathrm{AADH}$ on survival can be at least partly attributed to protection of organs since AADH administration reduced acute lung injury, decreased neutrophil influx and cytokine production in the lungs and protected against intrapulmonary protein leakage and capillary-alveolar leakage. Additionally, the viability of liver cells was significantly increased by AADH administration.

AADH administration had only moderately and short termed effects on thrombin generation and tail bleeding times that rapidly normalized after 3-6 hours (Figure 3D, Figure 4A-B, chapter 7). This residual anticoagulant effect of AADH can most likely be attributed to a stimulation of heparin cofactor II. The plasma concentration of this anticoagulant cofactor of $\sim 1 \mu \mathrm{M}(69,70)$ does however not allow complete inhibition of thrombin generation (given a prothrombin plasma concentration of $\sim 1.5 \mu \mathrm{M}$ (7173). The mice in our experiments were carefully checked for signs of bleedings, but in none of the AADH treated mice bleeding complications were observed.

In conclusion, we propose that AADH can be beneficial for the treatment of sepsis and other hyperinflammatory conditions that are characterized by excessive NET formation and extracellular histone levels. Based on extensive clinical experience available on the use of UFH and the observation that AADH did not induce bleedings in mice, we expect that AADH can be safely used in future clinical studies.

\section{Overall conclusions and future perspectives}

This thesis demonstrates that by careful study of their structure-function, the anticoagulant properties of the biomolecules APC and heparin can be manipulated, without affecting their anti-inflammatory, anti-apoptotic, endothelial barrier stabilizing and histone-neutralizing properties. Employing the 3D structure of APC, an exosite at the APC surface that is exclusively important for the anticoagulant function of APC can be targeted through a combination of computational and biochemical methods, by small molecules to increase in vivo thrombin generation for the potential benefit of individuals with bleeding tendencies, such as in hemophilia A patients. Administration 
of exogenous APC and heparin biomolecules that have greatly diminished anticoagulant properties, effectively reduced infarct size in a murine acute myocardial I/R injury model and protected organs and increased survival in three different murine models mimicking human sepsis. The great advantage of biomolecules that possess reduced anticoagulant properties is that they can safely be administered in patients who are already at risk for bleeding and that the dose can safely be raised in other patients to increase the therapeutic potential even further.

It is very conceivable that APC and heparin molecules with reduced anticoagulant properties will be further optimized in the near future to increase their cytoprotective properties. Since these molecules can provide cell-protection without causing bleeding complications the prospect of starting clinical studies exploring the efficacy of optimized molecules in patients suffering from hyper-inflammatory diseases such as sepsis is a realistic and attractive opportunity to translate the current state-of-the-art on APC and heparin into novel life-saving pharmacotherapeutic products. 


\section{References}

1. Sun YH, Shen L, Dahlbäck B (2003) Gla domain-mutated human protein C exhibiting enhanced anticoagulant activity and increased phospholipid binding. Blood 101: 2277-2284.

2. Sun Y, Tran S, Norstrøm E, Dahlbäck B (2004) Enhanced rate of cleavage at Arg-306 and Arg-506 in coagulation factor Va by gla domain-mutated human-activated protein C. J Biol Chem 279: 4752847535.

3. Preston R, Villegas-Mendez A, Sun Y, Hermida J, Simioni P, et al. (2005) Selective modulation of protein C affinity for EPCR and phospholipids by Gla domain mutation. FEBS J 272: 97-108.

4. Lee D, Walker I, Teitel J, Poon M, Ritchie B, et al. (2000) Effect of the factor V Leiden mutation on the clinical expression of severe hemophilia A. Thromb Haemost 83: 387-391.

5. Nowak-Göttl U, Escuriola C, Kurnik K, Schobess R, Horneff S, et al. (2003) Haemophilia and thrombophilia. What do we learn about combined inheritance of both genetic variations? Hamostaseologie 23: 36-40.

6. Gale A, Tsavaler A, Griffin J (2002) Molecular characterization of an extended binding site for coagulation factor Va in the positive exosite of activated protein C. J Biol Chem 277: 28836-28840.

7. Friedrich U, Nicolaes G, Villoutreix B, Dahlbäck B (2001) Secondary substrate-binding exosite in the serine protease domain of activated protein $\mathrm{C}$ important for cleavage at Arg-506 but not at Arg-306 in factor Va. J Biol Chem 276: 2305-2308.

8. Nicolaes G, Bock P, Segers K, Wildhagen K, Dahlbäck B, et al. (2010) Inhibition of Thrombin Formation by Active Site Mutated (S360A) Activated Protein C. J Biol Chem 285: 22890-22900.

9. Segers K, Dahlbäck B, Rosing J, Nicolaes G (2008) Identification of surface epitopes of human coagulation factor Va that are important for interaction with activated protein $\mathrm{C}$ and heparin. J Biol Chem 283: 22573-22581.

10. Nicolaes G, Sørensen K, Friedrich U, Tans G, Rosing J, et al. (2004) Altered inactivation pathway of factor Va by activated protein $C$ in the presence of heparin. Eur J Biochem 13: 2724-2736.

11. Gal S, Amontov S, Urvil P, Vishnuvardhan D, Nishikawa F, et al. (1998) Selection of a RNA aptamer that binds to human activated protein $\mathrm{C}$ and inhibits its protease function. Eur J Biochem 252: 553562.

12. Butenas S, Orfeo T, Kalafatis M, Mann KG (2006) Peptidomimetic inhibitors for activated protein C: implications for hemophilia management. J Thromb Haemost 4: 2411-2416.

13. De Nanteuil G, Gloanec P, Béguin S, Giesen PLA, Hemker HC, et al. (2006) Low molecular weight activated protein $\mathrm{C}$ inhibitors as a potential treatment for hemophilic disorders. J Med Chem 49: 50475050.

14. Müller J, Isermann B, Dücker C, Salehi M, Meyer M, et al. (2009) An exosite-specific ssDNA aptamer inhibits the anticoagulant functions of activated protein $\mathrm{C}$ and enhances inhibition by protein $\mathrm{C}$ inhibitor. Chem Biol 16: 442-451.

15. Scognamiglio P, Di Natale C, Perretta G, Marasco D (2013) From peptides to small molecules: an intriguing but intricated way to new drugs. Curr Med Chem 20: 3803-3817.

16. Bae J, Rezaie A (2008) Protease activated receptor 1 (PAR-1) activation by thrombin is protective in human pulmonary artery endothelial cells if endothelial protein $\mathrm{C}$ receptor is occupied by its natural ligand. Thromb Haemost 100: 101-109.

17. Mosnier L, Sinha R, Burnier L, Bouwens E, Griffin J (2012) Biased agonism of protease-activated receptor 1 by activated protein $C$ caused by noncanonical cleavage at Arg46. Blood 120: 5237-5246.

18. Bae J, Yang L, Manithody C (2007) The ligand occupancy of endothelial protein C receptor switches the PAR-1-dependent signaling specificity of thrombin from a permeability-enhancing to a barrierprotective response in endothelial cells. Blood 110: 3909-3916. 
19. Bae JS, Rezaie AR (2009) Thrombin inhibits nuclear factor $\mathrm{kB}$ and RhoA pathways in cytokinestimulated vascular endothelial cells when EPCR is occupied by protein C. Thromb Haemost 101: 513-520.

20. Gale AJ, Sun X, Heeb MJ, Griffin JH (1997) Nonenzymatic anticoagulant activity of the mutant serine protease Ser360Ala-activated protein C mediated by factor Va. Protein science 6: 132-140.

21. Hirose K, Okajima K, Taoka Y, Uchiba M, Tagami H, et al. (2000) Activated protein C reduces the ischemia/reperfusion-induced spinal cord injury in rats by inhibiting neutrophil activation. Ann Surg 232: $272-280$.

22. Loubele ST, Spek CA, Leenders P, van Oerle R, Aberson HL, et al. (2009) Activated protein C protects against myocardial ischemia/reperfusion injury via inhibition of apoptosis and inflammation. Arterioscler Thromb Vasc Biol 29: 1087-1092.

23. Wang Y, Sinha R, Mosnier L, Griffin J, Zlokovic B (2013) Neurotoxicity of the anticoagulant-selective E149A-activated protein C variant after focal ischemic stroke in mice. Blood Cells Mol Dis 51: 104108.

24. Guo H, Wang Y, Singh I, Liu D, Fernandez JA, et al. (2009) Species-dependent neuroprotection by activated protein $C$ mutants with reduced anticoagulant activity. J Neurochem 109: 116-124.

25. Krisinger M, Guo LJ, Salvagno G, Guidi GC, Lippi G, et al. (2009) Mouse recombinant protein C variants with enhanced membrane affinity and hyper-anticoagulant activity in mouse plasma. FEBS J 276: 6586-6602.

26. Guo H, Liu D, Gelbard H, Cheng T, Insalaco R, et al. (2004) Activated protein C prevents neuronal apoptosis via protease activated receptors 1 and 3. Neuron 41: 563-572.

27. Zhong Z, Ilieva H, Hallagan L, Bell R, Singh I, et al. (2009) Activated protein C therapy slows ALS-like disease in mice by transcriptionally inhibiting SOD1 in motor neurons and microglia cells. J Clin Invest 119: 3437-3449.

28. Thiyagarajan M, Fernández J, Lane S, Griffin J, Zlokovic B (2008) Activated protein C promotes neovascularization and neurogenesis in postischemic brain via protease-activated receptor $1 . \mathrm{J}$ Neurosci 28: 12788-12797.

29. Xu J, Zhang X, Pelayo R, Monestier M, Ammollo C, et al. (2009) Extracellular histones are major mediators of death in sepsis. Nat Med 15: 1318-1321.

30. Mosnier LO, Yang XV, Griffin JH (2007) Activated protein C mutant with minimal anticoagulant activity, normal cytoprotective activity, and preservation of thrombin activable fibrinolysis inhibitordependent cytoprotective functions. J Biol Chem 282: 33022-33033.

31. Wildhagen K, Lutgens E, Loubele S, ten Cate H, Nicolaes G (2011) The structure-function relationship of activated protein C. Lessons from natural and engineered mutations. Thromb Haemost 106: 10341045.

32. Bakker H, Tans G, Janssen-Claessen T, Thomassen M, Hemker H, et al. (1992) The effect of phospholipids, calcium ions and protein $\mathrm{S}$ on rate constants of human factor Va inactivation by activated human protein C. Eur J Biochem 208: 171-178.

33. Baker S, Worthley L (2002) The Essentials of Calcium, Magnesium and Phosphate Metabolism: Part I. Physiology Crit Care Resusc 4: 301-306.

34. Wang Y, Chen Y, Xin L, Beverley S, Carlsen E, et al. (2011) Differential microbicidal effects of human histone proteins $\mathrm{H} 2 \mathrm{~A}$ and $\mathrm{H} 2 \mathrm{~B}$ on Leishmania promastigotes and amastigotes. Infect Immun 79: $1124-1133$.

35. Hirsch J (1958) Bactericidal action of histone. J Exp Med 108: 925-944.

36. Zhdan-Pushkina S, Dronova N (1976) Differential sensitivity of bacteria to histones. Mikrobiologiia 45: 60-66. 
37. Rosenbluh J, Hariton-Gazal E, Dagan A, Rottem S, Graessmann A, et al. (2005) Translocation of histone proteins across lipid bilayers and Mycoplasma membranes. J Mol Biol 345: 387-400.

38. Kleine T, Lewis P, Lewis S (1997) Histone-induced damage of a mammalian epithelium: the role of protein and membrane structure. Am J Physiol 273: C1925-1936.

39. Pereira L, Marco F, Boimorto R, Caturla A, Bustos A, et al. (1994) Histones interact with anionic phospholipids with high avidity; its relevance for the binding of histone-antihistone immune complexes. Clin Exp Immunol 97: 175-180.

40. Rosing J, Hoekema L, Nicolaes G, Thomassen M, Hemker H, et al. (1995) Effects of protein S and factor Xa on peptide bond cleavages during inactivation of factor Va and factor VaR506Q by activated protein C. J Biol Chem 270: 27852-27858.

41. Nicolaes G, Tans G, Thomassen M, Hemker H, Pabinger I, et al. (1995) Peptide bond cleavages and loss of functional activity during inactivation of factor Va and factor VaR506Q by activated protein C. J Biol Chem 270: 21158-21166.

42. Smirnov M, Esmon C (1994) Phosphatidylethanolamine incorporation into vesicles selectively enhances factor Va inactivation by activated protein C. J Biol Chem 269: 816-819.

43. Norstrøm E, Steen M, Tran S, Dahlbäck B (2003) Importance of protein S and phospholipid for activated protein C-mediated cleavages in factor Va. J Biol Chem 278: 24904-24911.

44. Taylor Fj, Chang A, Esmon C, D'Angelo A, Vigano-D'Angelo S, et al. (1987) Protein C prevents the coagulopathic and lethal effects of Escherichia coli infusion in the baboon. J Clin Invest 79: 918-925.

45. Zeerleder S, Stephan F, Emonts M, de Kleijn E, Esmon C, et al. (2012) Circulating nucleosomes and severity of illness in children suffering from meningococcal sepsis treated with protein C. Crit Care Med 40: 3224-3229.

46. Zeerleder S, Zwart B, Wuillemin W, Aarden L, Groeneveld A, et al. (2003) Elevated nucleosome levels in systemic inflammation and sepsis. Crit Care Med 31: 1947-1951.

47. Chen Q, Ye L, Jin Y, Zhang N, Lou T, et al. (2012) Circulating nucleosomes as a predictor of sepsis and organ dysfunction in critically ill patients. Int J Infect Dis 16: e558-564.

48. Kutcher M, Xu J, Vilardi R, Ho C, Esmon C, et al. (2012) Extracellular histone release in response to traumatic injury: implications for a compensatory role of activated protein C. J Trauma Acute Care Surg 73: 1389-1394.

49. Fuchs T, Bhandari A, Wagner D (2011) Histones induce rapid and profound thrombocytopenia in mice. Blood 118: 3708-3714.

50. Semeraro F, Ammollo C, Morrissey J, Dale G, Friese P, et al. (2011) Extracellular histones promote thrombin generation through platelet-dependent mechanisms: involvement of platelet TLR2 and TLR4. Blood 118: 1952-1961.

51. Carestia A, Rivadeneyra L, Romaniuk M, Fondevila C, Negrotto S, et al. (2013) Functional responses and molecular mechanisms involved in histone-mediated platelet activation. Thromb Haemost 110: 1035-1045.

52. Allam R, Darisipudi M, Tschopp J, Anders H (2013) Histones trigger sterile inflammation by activating the NLRP3 inflammasome. Eur J Immunol 43: 3336-3342.

53. Schroder K, Tschopp J (2010) The inflammasomes. Cell 140: 821-832.

54. Xu J, Zhang X, Monestier M, Esmon N, Esmon C (2011) Extracellular histones are mediators of death through TLR2 and TLR4 in mouse fatal liver injury. J Immunol 187: 2626-2631.

55. Allam R, Scherbaum C, Darisipudi M, Mulay S, Hagele H, et al. (2012) Histones from dying renal cells aggravate kidney injury via TLR2 and TLR4. J Am Soc Nephrol 23: 1375-1388.

56. Wildhagen K, García de Frutos P, Reutelingsperger C, Schrijver R, Aresté C, et al. (2014) Nonanticoagulant heparin prevents histone-mediated cytotoxicity in vitro and improves survival in sepsis. Blood 123: 1098-1101. 
57. Van Dreden P, Woodhams B, Rousseau A, Dreyfus J, Vasse M (2013) Contribution of procoagulant phospholipids, thrombomodulin activity and thrombin generation assays as prognostic factors in intensive care patients with septic and non-septic organ failure. Clin Chem Lab Med 51: 387-396.

58. Collins P, Macchiavello L, Lewis S, Macartney N, Saayman A, et al. (2006) Global tests of haemostasis in critically ill patients with severe sepsis syndrome compared to controls. Br J Haematol 135: 220-227.

59. Petros S, Kliem P, Siegemund T, Siegemund R (2012) Thrombin generation in severe sepsis. Thromb Res 129: 797-800.

60. Massion P, Peters P, Ledoux D, Zimermann V, Canivet J, et al. (2012) Persistent hypocoagulability in patients with septic shock predicts greater hospital mortality: impact of impaired thrombin generation. Intensive Care Med 38: 1326-1335.

61. Levi M, de Jonge E, van der Poll T (2003) Sepsis and disseminated intravascular coagulation. J Thromb Thrombolysis 16: 43-47.

62. Levi M, Van der Poll T (2005) Two-way interactions between inflammation and coagulation. Trends Cardiovasc Med 15: 254-259.

63. Phypers B, Pierce J (2006) Lactate physiology in health and disease. Anaesthesia, Critical Care \& Pain 6: $128-132$.

64. Gibot S (2012) On the origins of lactate during sepsis. Crit Care 16: 151.

65. Zein J, Lee G, Tawk M, Dabaja M, Kinasewitz G (2004) Prognostic Significance of Elevated Serum Lactate Dehydrogenase (LDH) in Patients with Severe Sepsis. Chest 126: 873S.

66. Londoño J, León A, Rodríguez F, Barrera L, de la Rosa G, et al. (2013) Serum lactate in the emergency department as a prognostic factor in patients with sepsis without hypotension. Med Clin (Barc) 141: 246-251.

67. Marty P, Roquilly A, Vallée F, Luzi A, Ferré F, et al. (2013) Lactate clearance for death prediction in severe sepsis or septic shock patients during the first 24 hours in Intensive Care Unit: an observational study. Ann Intensive Care 3.

68. Grommes J, Alard J, Drechsler M, Wantha S, Mörgelin M, et al. (2012) Disruption of platelet-derived chemokine heteromers prevents neutrophil extravasation in acute lung injury. Am J Respir Crit Care Med 185: 628-636.

69. Sie P, Dupouy D, Pichon J, Boneu B (1985) Constitutional heparin co-factor II deficiency associated with recurrent thrombosis. Lancet 2: 414-416.

70. Tollefsen D, Pestka C (1985) Heparin cofactor II activity in patients with disseminated intravascular coagulation and hepatic failure. Blood 66: 769-774.

71. Mann K (1976) Prothrombin. Methods Enzymol 45: 123-156.

72. Koldas M, Uras F (2001) Avidin-biotin ELISA for measurement of prothrombin in human plasma. Thromb Res 102: 221-227.

73. Shapiro S, Martinez J (1969) Human prothrombin metabolism in normal man and in hypocoagulable subjects. J Clin Invest 48: 1292-1298. 
Nederlandse Samenvatting 
In hoofdstuk 1 wordt het onderwerp van dit proefschrift geïntroduceerd. De extrinsieke en intrinsieke bloedstolling en de verschillende functies van geactiveerd proteïne $C$ (APC) en heparine worden beschreven. Beide biomoleculen bezitten naast hun antistollende eigenschappen ook celbeschermende functies en zijn in staat om de schadelijke effecten van histonen te neutraliseren. In vivo toediening van zowel APC als heparine kan echter bloedingscomplicaties veroorzaken. In hoofdstuk 1 zijn daarom varianten van APC en heparine beschreven die sterk verminderde antistollende eigenschappen hebben. De celbeschermende eigenschappen zijn intact gelaten en onderzocht in atherosclerose, hartinfarct en sepsis muismodellen.

De relatie tussen de structuur en functie van APC wordt besproken in hoofdstuk 2. APC bestaat uit verschillende domeinen: een $\gamma$-carboxyglutamaat (Gla)-domein, twee epidermale groei factor (EGF) domeinen, een verbindingspeptide en een trypsinegelijkend serine protease domein. Onderzoek naar zowel natuurlijk voorkomende APC varianten, als naar varianten waarin door onderzoekers bewust mutaties aangebracht zijn, heeft veel informatie opgeleverd over de relatie tussen de structuur en de functie van APC. Hierdoor is het mogelijk geworden om de verschillende functies van APC te koppelen aan specifieke gebieden in de 3D structuur. Niet alle beschreven APC varianten zijn tot op heden onderzocht op hun celbeschermende effecten, noch zijn alle mogelijke varianten gemaakt, dus mogelijk worden in de toekomst nog meer aminozuren geïdentificeerd die belangrijk zijn voor de celbeschermende functie van APC. Dit zal helpen bij het ontwerpen van nieuwe APC mutanten die niet antistollend en in verhoogde mate celbeschermend zijn en daardoor een optimale celbescherming bieden in een aantal klinisch relevante condities, zonder dat ze een verhoogd risico op bloedingscomplicaties geven.

Verschillende in vitro en in vivo waarnemingen suggereren dat resistentie tegen APC of een verminderde activiteit van APC geassocieerd zijn met een verschuiving van de hemostatische balans naar een meer procoagulante staat, doordat FVa in plasma minder geremd wordt waardoor meer trombine gevormd kan worden. Remming van APC zou dus hypothetisch bij kunnen dragen aan het verminderen van de bloedingsneiging in hemofilie A patiënten. In hoofdstuk $\mathbf{3}$ is een gevalideerde in silico structuur-gedreven virtuele screenings methode gebruikt voor de selectie van kleine moleculen die specifiek de interactie tussen APC en FVa remmen, zonder dat ze de celbeschermende effecten van APC beïnvloeden. Na in silico selectie zijn 624 kleine moleculen in een functionele test onderzocht op hun vermogen om de inactivatie van FVa door APC te kunnen remmen. Van de 20 beste compounds is vervolgens de bindingsaffiniteit en -specificiteit bepaald. Verdere optimalisatie en ontwikkeling van de beste kleine moleculen is nodig om hun affiniteit en functionele activiteit te verbeteren, zodat ze effectief gebruikt 
kunnen worden om in vivo trombine generatie te verhogen in individuen met een bloedingsneiging, zoals hemofilie A patiënten.

In hoofdstuk 4 zijn de celbeschermende effecten van wild type (wt)-APC en de verminderd antistollende S360A-APC variant onderzocht in een acuut hartinfarct model en een chronisch atherosclerose model, waarbij zowel het enzym als het zymogeen van deze twee APC varianten is getest. Zowel wt-PC, S360A-PC, wt-APC en S360AAPC reduceerden de grootte van het geïnfarcteerde gebied in het hart in gelijke mate, maar hadden geen effect op IL-6 concentraties in het hart. De S360A-varianten zijn niet in staat om FVa, FVIIIa en PAR-1 proteolytisch te splitsen, dus mechanismen onafhankelijk van de actieve site moeten een rol hebben gespeeld bij het gevonden beschermende effect. Mogelijk hebben de varianten een dissociatie tussen EPCR en caveolin-1 geïnitieerd, waarna proteolyse van PAR-1 door zowel trombine als APC voor anti-inflammatoire signalering zorgt. Daarnaast kunnen alle varianten via hun Gladomein een interactie aangegaan zijn met fosfolipiden in LDL deeltjes en daardoor de productie van reactieve zuurstofradicalen en de oxidatie van vetten verminderd hebben. Het uitblijven van een effect van APC op IL-6 concentraties kan verklaard worden door de lage concentratie IL-6 in onze placebo groep of doordat wij humaan APC toegediend hebben in plaats van muizen APC. Het is bekend dat muizen APC een iets hogere affiniteit heeft voor de muizen PAR-1 receptor dan humaan APC. Wij zijn van mening dat voor toediening in een klinische setting, 360A-(A)PC geschikter is dan wt(A)PC, omdat de in vivo halfwaardetijd van 360A-(A)PC langer is en deze variant de kans op bloedingscomplicaties reduceert.

Vervolgens is het effect van dezelfde (A)PC varianten op het ontstaan van atherosclerose onderzocht. Helaas werd in geen van de behandelgroepen een effect op de grootte van atherosclerotische plaques gevonden. Sommige varianten hadden wel effect op de samenstelling van de atherosclerotische plaques en zowel S360A-PC en wt-APC zorgden voor verlaagde IL-6 plasma concentraties. Waarschijnlijk was de biobeschikbaarheid van (A)PC te gelimiteerd om het chronische proces van plaque ontwikkeling te kunnen beïnvloeden. Wij geloven dat de varianten in potentie ook het ontstaan van atherosclerose kunnen beïnvloeden als voor een hogere biobeschikbaarheid van APC gezorgd kan worden.

Recentelijk is ontdekt dat APC de capaciteit heeft om extracellulaire histonen te knippen en daarmee onschadelijk te maken. In hoofdstuk $\mathbf{5}$ is het effect van calcium en fosfolipiden op de proteolytische inactivatie van histonen door zowel wt-als 5A-APC onderzocht. 5A-APC heeft een normale celbeschermende- maar minimale antistollende activiteit. 5A-APC knipt histon H3 significant sneller dan wt-APC en beschermt hierdoor endotheelcellen beter tegen de cytotoxische effecten van histonen. De verklaring hiervoor is waarschijnlijk dat de affiniteit van deze variant voor sterk positief geladen 
histonen verhoogd is, doordat 5 positief geladen aminozuren door neutrale aminozuren vervangen zijn. Optimale proteolyse van histon $\mathrm{H} 3$ vindt plaats in aanwezigheid van fysiologische calcium concentraties en van fosfolipiden vesikels die zowel PS, PE en PC bevatten. Wt-APC en 5A-APC knippen histon H3 beiden op dezelfde posities. Door middel van Edman sequencing is vastgesteld dat de belangrijkste knipplaatsen C-terminaal van aminozuren R26 en R52 liggen. De resulterende eindproducten die ontstaan door deze proteolyse zijn niet meer cytotoxisch. APC zou dus in de toekomst mogelijk ingezet kunnen worden bij de behandeling van pathologische condities waarbij verhoogde concentraties extracellulaire histonen voorkomen, zoals bijvoorbeeld sepsis en trauma. 5A-APC heeft de voorkeur boven wt-APC omdat deze variant histonen efficiënter proteolyseert en een verminderd bloedingsrisico geeft in vergelijking met wt-APC.

In patiënten met systemische ontsteking en sepsis zijn verhoogde concentraties extracellulaire histonen gevonden, die direct celdood kunnen induceren door schade toe te brengen aan celmembranen en indirect celdood kunnen veroorzaken via activatie van TLR2- en TLR4-signalering en NLRP3 inflammasomen. Deze celdood zorgt voor verdere vrijkomst van histonen, waardoor een vicieuze cirkel ontstaat. Histonen in de circulatie kunnen daarnaast afkomstig zijn van NETs die door neutrofielen gemaakt worden om infecties te bestrijden. In hoofdstuk 6 zijn in plasma samples van 43 sepsis patiënten histon $\mathrm{H} 3$ concentraties gemeten en mogelijke correlaties met de ernst van de ziekte, mortaliteit en weefselschade- en stollingsparameters onderzocht. Niet-overlevenden bleken significant hogere concentraties histon $\mathrm{H} 3$ in hun plasma te hebben dan patiënten die de ziekte overleefden. Extracellulaire histon $\mathrm{H} 3$ concentraties correleerden significant positief met de mate van sepsis-geïnduceerd orgaanfalen en weefselschadeparameters (lactaat dehydrogenase activiteit en lactaat concentratie). Significant negatieve correlaties waren aanwezig tussen histon $\mathrm{H} 3$ concentraties en de hoeveelheid bloedplaatjes en de antitrombine concentratie. De ontwikkeling en progressie van sepsis is een multi-factorieel proces. We hebben laten zien dat er correlaties zijn tussen de hoeveelheid extracellulaire histonen en weefselschade- en stollingsparameters. Deze reflecteren allemaal de ernst van de systemische ontsteking, stollingsproblemen en orgaanfalen en kunnen dus mogelijk gebruikt worden als biomarkers om de ernst van de ziekte en mortaliteit te voorspellen.

Heparine is door zijn sterke negatieve lading een goed molecuul om door middel van de binding aan positief geladen histonen, de schadelijke effecten van deze laatsten te neutraliseren. Het nadeel van heparine is echter dat het als complicatie bloedingen kan veroorzaken en daarom niet veilig toe te dienen is aan sepsis patiënten die vaak al bloedstollingsproblemen hebben. We hebben daarom in hoofdstuk 7 een nietantistollend heparine molecuul ontwikkeld en onderzocht: antitrombine affiniteit- 
gedepleteerd heparine (AADH). In vitro heeft AADH hetzelfde beschermende effect op histon-geïnduceerde celdood als UFH. We hebben aangetoond dat AADH een bindingsaffiniteit van $86 \mathrm{nM}$ heeft voor histonen en co-lokaliseert met histonen die in NETs geïncorporeerd zijn. Vervolgens zijn de effecten van AADH in 3 verschillende in vivo muismodellen onderzocht. In het ConA-geïnduceerde steriele ontstekingsmodel model hebben we gezien dat toediening van AADH op korte termijn de vrijkomst van histonen remt. Op de langere termijn was er geen onderscheid tussen de concentraties extracellulaire histonen in de controle- en AADH-behandelde groep, maar waren de overlevingskansen in de AADH-behandelde groep door complexatie van de histonen toch duidelijk verhoogd. Ook in het CLP sepsis model en in het LPS septische shock model zorgde behandeling met AADH voor significant lagere mortaliteit. Zelfs als $\mathrm{AADH}$ in een behandel-regime pas 4 uur na de inductie van sepsis toegediend werd, was de mortaliteit significant verlaagd. Het beschermende effect van AADH is op zijn minst deels toe te schrijven aan een verminderde incidentie van orgaanfalen. Behandeling met $\mathrm{AADH}$ vermindert de kans op acute longonsteking en zorgt voor een toename van de celviabiliteit in de lever.

$\mathrm{AADH}$ heeft alleen op korte termijn licht antistollende effecten die toe te schrijven zijn aan stimulatie van heparine cofactor II. Alle muizen zijn uitgebreid gecontroleerd op tekenen van bloedingen, maar in geen enkel geval werden bloedingscomplicaties gevonden. AADH kan dus veilig in vivo toegediend worden en een toegevoegde waarde hebben bij de behandeling van sepsis en andere ziekten waarin verhoogde concentraties extracellulaire histonen voorkomen. Vanwege het feit dat er al veel klinische ervaring is met de toediening van UFH en dat AADH geen bloedingen veroorzaakt, verwachten wij dat het in de toekomst veilig getest kan worden in klinische studies.

In hoofdstuk 8 worden de voornaamste bevindingen van dit proefschrift besproken. Dit proefschrift laat zien dat gedegen kennis van de structuur-functie relatie het mogelijk maakt om biomoleculen zodanig te manipuleren dat hun ongewenste antistollende effecten sterk beperkt worden, terwijl hun gewenste celbeschermende effecten behouden blijven of zelfs versterkt worden. Structuur-functie informatie maakt het ook mogelijk om met kleine moleculen selectief bepaalde functies van biomoleculen te remmen, zonder andere functies te verstoren.

APC en heparine varianten met sterk verminderde antistollende eigenschappen waren in staat infarctgroottes te verkleinen in een acuut hartinfarct model en te zorgen voor verminderde orgaanschade en een grotere overlevingskans in 3 verschillende ontstekingsmodellen. Het grote voordeel van deze varianten ten opzichte van de originele biomoleculen is dat de toegediende dosis, door hun verminderde risico op bloedingen, verhoogd kan worden, waardoor maximale doeltreffendheid bereikt kan worden. Hopelijk is het in de toekomst mogelijk deze varianten te testen in klinische studies en kunnen deze varianten bijdragen aan een verbetering van de huidige 
behandeling voor sepsis en andere klinische condities waarin verhoogde concentraties extracellulaire histonen voorkomen. 
Valorisation 


\section{Structure function relationship of activated protein C}

In Chapter 2 the structure-function relationship of activated protein C (APC) is described. APC is an anticoagulant protein that is essential for maintenance of a haemostatic balance. We have studied the influence of natural and engineered mutations in the PC gene on the function of APC. The most important functions of APC comprise its anticoagulant and its cytoprotective properties. Chapter 2 provides an overview of the effects of several mutations on the different functions of APC and links certain parts of the APC structure to specific functions. This information is interesting for individuals who have mutations in their PC gene and, as a result of a lower functional activity of (A)PC, are at an increased risk to develop venous thrombosis. Information about the structure-function relationship of PC provides both clinicians and patients additional insights in the mechanism(s) responsible for the pro-thrombotic tendency. When the mutation that causes a disease is known and it may become clear which particular function of PC is affected by a certain mutation, a better insight in the pathogenesis can be provided. Potentially a deepened understanding of the molecular mechanism may provide new insights for design of novel methods for prevention of venous thrombosis in this group of patients. Therefore we have published a Dutch summary of this review in the magazine of the Federation of Dutch Thrombosis Services (FNT). One of the aims of the FNT is to improve the quality of anticoagulant treatment for thrombosis patients. By providing their members with information about relevant scientific research, they keep the scientific knowledge of the medical doctors working at the thrombosis services up-to-date.

Worldwide, several research groups are working on the development of engineered (A)PC variants that are specifically tailored for application in certain treatments, such as in anticoagulation or anti-inflammatory treatments. Detailed information about the structure-function relation relationship of (A)PC is indispensable for the engineering of (A)PC variants without unwanted side effects and that provide optimal in vivo efficacy.

\section{Small molecule inhibitors of Activated Protein C}

APC is an attractive target for inhibition to provide support of in vivo thrombin generation in individuals where thrombin formation is compromised and the haemostatic balance is tipped towards bleeding, such as in hemophilia A patients. Hemophilia A is an X chromosome-linked bleeding disorder with an estimated incidence of 1 in 5000 male life births [1] that is caused by mutation of the FVIII gene. Hemophilia A is usually treated by intravenous infusions of FVIII concentrates. In spite of these infusions and advanced medical care, individuals with severe hemophilia regularly develop bleedings and musculoskeletal complications. Up to $30 \%$ of severe hemophilia A patients develop neutralizing antibodies to infused FVIII concentrates, making the infusions no longer useful for coagulation [2]. 
We discovered several small molecule inhibitors that are able to inhibit the interaction of APC with its natural substrate FVa with weak to moderate binding affinities. These molecules could function as starting molecules for further development into orally available small molecules that could eventually become of clinical interest for patients with hemophilia A.

\section{Activated protein C mutants}

According to the WHO [3], cardiovascular diseases are the number one cause of death worldwide. Yearly, 30\% of all deaths are related to cardiovascular diseases. Among cardiovascular diseases, mortality from ischemic heart disease is most frequent, with worldwide 7.4 million deaths per year. These numbers underline that improvement of treatment of cardiovascular diseases could have a great impact on society.

Activated protein C (APC) possesses cytoprotective effects that comprise in vitro antiinflammatory, anti-apoptoic and endothelial barrier stabilizing effects on different cell types $[4,5]$. Recently Xu et al. [6] discovered that APC can cleave histones in vitro and in vivo, providing an additional cytoprotective mechanism of action. APC provides protection in mouse models of ischemic/embolic stroke, myocardial infarction, sepsis and lung injury [7-12]. The disadvantage of in vivo administration of APC is that it is accompanied with an increased bleeding risk. Therefore we investigated the cytoprotective properties of engineered APC variants with limited anticoagulant properties, such as S360A-APC and 5A-APC.

In chapter 4, we investigated the effect of S360A-APC administration in a myocardial ischemia/reperfusion mouse model. This study showed that administration of equal doses of S360A-APC or wt-APC, resulted both in a two times reduced infarcted area in the heart. We hypothesize that S360A-APC can potentially reduce the infarcted area of the heart even further than wt-APC, because its dose of administration can be higher than that of wt-APC due to its reduced anticoagulant activity. We conclude that S360A has the potential to improve the treatment of ischemic heart disease in the future, but more data need to be generated before such improvements can indeed be verified.

In chapter 5 , we compared the in vitro proteolytic capacities of the low-anticoagulant 5A-APC variant towards histones with those of wt-APC and found that 5A-APC proteolyzes histones more efficiently than wt-APC. From this observation we conclude that 5A-APC can potentially be used to treat sepsis and other diseases that are accompanied with cell death and/or NET formation in the future.

\section{Antithrombin affinity-depleted heparin}

Sepsis is a severe complication to infections, with an estimated worldwide incidence rate of at least 19 million cases per year [13]. The incidence of sepsis increases with age [14], and due to increasing life expectancies, the incidence rate of sepsis is expected to 
increase even further in the near future [15]. In order to tackle an infection, the human immune system initiates several different strategies, one of these is the production of neutrophil extracellular traps (NETs). These NETs do unfortunately not only kill bacteria, but are in addition cytotoxic to the bodies' own cells, resulting in the release of additional histones into the blood stream. Extracellular histones damage the cell walls of internal organs, resulting in a combination of thrombosis, internal bleedings and inflammatory reactions. When one or more organs are failing due to this combination of complications, this can evolve into a life threatening condition that is very difficult to treat. Despite the use of potent antibiotics and advances made in supportive therapy for failing organs, the mortality rates of severe sepsis and septic shock are still around $30 \%[16]$.

Heparin has a strong affinity for positively charged histones $[17,18]$ due to its extremely high negative charge density ratio [19]. Therefore we hypothesized that heparin could possibly neutralize in vivo histone-induced cytotoxicity. In a clinital trial low dose unfractionated heparin (UFH) has been administered as a complementary treatment of sepsis [20]. The authors of this study linked the presence of infection, inflammation and coagulation in sepsis and aimed to inhibit the coagulation part with low doses of heparin so as not to increase the bleeding risk in these patients who are already at risk due to sepsis-associated consumption coagulopathy [20,21]. Although this study failed to demonstrate a significant benefit on 28-day mortality rate, we believe that the minor beneficial effects of heparin observed might be attributed to its histoneneutralizing capacity and were independent of its anticoagulant properties. To test this hypothesis we used affinity chromatography to eliminate the anticoagulant properties of unfractionated heparin (UFH), without interfering with its capacity to bind and neutralize histones. The resulting product, antithrombin affinity-depleted heparin (AADH), has only an estimated $0.2 \%$ to $0.5 \%$ of the anticoagulant activity of UFH and accordingly $\mathrm{AADH}$ has the advantage that the administered concentration can be safely increased to enhance the in vivo capacity to bind and eliminate histones. We tested the in vivo cytoprotective capacity of $\mathrm{AADH}$ in three different sepsis mouse models and found that AADH significantly limited organ failure, the most dangerous complication of bacterial infections, and thereby increased survival in all models.

Maastricht University has filed a patent application based on the key results of our research that claims the use of pentasaccharide-depleted heparin in the treatment or prevention of sepsis, SIRS, severe sepsis or septic shock (European Patent Office (EP2731616 A1), Patent Cooperation Treaty or PCT (WO2013007771 A1), United States Patent and Trademark Office (US20140162978 A1) and the Canadian Intellectual Property Office (CA2842060 A1)). The patent application will help to develop AADH into a clinical product and to monetize the invention. 


\section{References}

1. Mannucci, P. and E. Tuddenham, The hemophilias--from royal genes to gene therapy. N Engl J Med, 2001. 344(23): p. 1773-9.

2. Srivastava, A., et al., Guidelines for the management of hemophilia. Haemophilia, 2013. 19(1): p. e1-e47.

3. Organization, W.H. The top 10 causes of death, Fact sheet $N^{\circ} 310.2012$; Available from: http://www.who. int/mediacentre/factsheets/fs310/en/.

4. Bae, J., L. Yang, and A. Rezaie, Receptors of the protein $C$ activation and activated protein $C$ signaling pathways are colocalized in lipid rafts of endothelial cells. Proc Natl Acad Sci USA, 2007. 104: p. 2867-72.

5. Mosnier, L.O., B.V. Zlokovic, and J.H. Griffin, The cytoprotective protein C pathway. Blood, 2007. 109: p. 3161-72.

6. $\mathrm{Xu}, \mathrm{J}$, et al., Extracellular histones are major mediators of death in sepsis. Nat Med, 2009. 15(11): p. 1318-21.

7. Murakami, K., et al., Activated protein $C$ attenuates endotoxin-induced pulmonary vascular injury by inbibiting activated leukocytes in rats. Blood, 1996. 87: p. 642-7.

8. Zlokovic, B., et al., Functional recovery after embolic stroke in rodents by activated protein C. Ann Neurol, 2005. 58: p. 474-7.

9. Loubele, S.T., et al., Activated protein $C$ protects against myocardial ischemia/ reperfusion injury via inbibition of apoptosis and inflammation. Arterioscler Thromb Vasc Biol, 2009. 29: p. 1087-92.

10. Fernandez, J.A., et al., Recombinant murine activated protein $C$ is neuroprotective in a murine ischemic stroke model. Blood Cells Mol Diseases, 2003. 30: p. 271-6.

11. Taylor, F.j., et al., Protein C prevents the coagulopathic and lethal effects of Escherichia coli infusion in the baboon. J Clin Invest, 1987. 79: p. 918-25.

12. Shibata, M., et al., Anti-inflammatory, antithrombotic and neuroprotective effects of activated protein $C$ in a murine model of focal ischemic stroke. Circulation, 2001. 103: p. 1799-1805.

13. Adhikari, N., et al., Critical care and the global burden of critical illness in adults. Lancet, 2010. 376(9749): p. 1339-46.

14. Martin, G., D. Mannino, and M. Moss, The effect of age on the development and outcome of adult sepsis. Crit Care Med, 2006. 34(1): p. 15-21.

15. Angus, D., et al., Epidemiology of severe sepsis in the United States: analysis of incidence, outcome, and associated costs of care. Crit Care Med, 2001. 29: p. 1303-1310.

16. Angus, D. and T. van der Poll, Severe sepsis and septic shockvere sepsis and septic shock. N Engl J Med, 2013. 369(9): p. 840-51.

17. Kent, P., M. Hichens, and P. Ward, Displacement fractionation of deoxyribonucleoproteins by beparin and dextran sulphate. Biochem J, 1958. 68(4): p. 568-72.

18. Demidenko, O. and S. Tsvetkova, Action of heparin on protein fractions of isolated nuclei and on their DNA content. . Histochemistry, 1978. 57(3): p. 265-71.

19. Cox, M. and D. Nelson, Lehninger, Principles of Biochemistry. 2008, W.H. Freeman \& Company. p. 1158.

20. Jaimes, F., et al., Unfractioned heparin for treatment of sepsis: A randomized clinical trial (The HETRASE Study). Crit Care Med, 2009. 37(4): p. 1185-96.

21. Dhainaut, J., et al., Dynamic evolution of coagulopatby in the first day of severe sepsis: relationship with mortality and organ failure. Crit Care Med, 2005. 33(2): p. 341-8. 

Dankwoord 
Dit proefschrift was nooit tot stand gekomen zonder de hulp van vele mensen zowel binnen als buiten de Universiteit Maastricht. Ik kijk met veel plezier terug op de afgelopen jaren dat ik bij de afdeling Biochemie gewerkt heb en zal de gezelligheid en de borrels in de toekomst zeker gaan missen. Ik wil iedereen die betrokken is geweest bij mijn promotie-traject hartelijk bedanken, ook degenenen die hieronder niet bij naam genoemd worden.

Dr. Nicolaes, beste Gerry, ik ben blij dat jij me de kans geboden hebt om na mijn masterstage als $\mathrm{PhD}$-student aan de slag te gaan. Je deur stond te allen tijde voor me open voor vragen en voor discussie van resultaten van experimenten. Als ik hulp nodig had bij teksten voor papers of bij presentaties en posters die ik voorbereidde, dan maakte je daar altijd zo snel mogelijk tijd voor en voorzag me van uitgebreide feedback. Dat heeft in grote mate bijgedragen aan mijn ontwikkeling als wetenschapper en de voltooiing van dit proefschrift. Buiten het werk heb ik goede herinneringen aan de Sinterklaasavonden die je samen met je vrouw Silvie ieder jaar organiseerde bij jou thuis.

Prof. dr. Hackeng, beste Tilman, ik wil je bedanken voor alle suggesties en adviezen die je hebt gegeven tijdens de werkbesprekingen op donderdagochtend en daarbuiten. Hierdoor heb ik vanuit een andere invalshoek naar mijn onderzoek leren kijken en dit heeft me erg geholpen bij het tot stand komen van mijn proefschrift. Als vakgroepvoorzitter heb je een belangrijke bijdrage geleverd aan de ontwikkeling van de afdeling Biochemie. Door je optimistische houding was het altijd fijn om met je te praten. Ik heb goede herinneringen aan de openingen van het academisch jaar die bij jou thuis in de tuin gehouden werden en aan de vele belevenissen die we in India gezamenlijk meegemaakt hebben.

Prof. dr. Reutelingsperger, beste Chris, dankzij jou heb ik een hoop bijgeleerd over verschillende manieren van celdood (apoptose, necrose, necroptose) en de methoden die gebruikt kunnen worden om deze te onderzoeken. Je betrokkenheid bij het AADH project heeft in mijn ogen in belangrijke mate bijgedragen aan het succes van dit project. Ik wil je bedanken voor de tijd die je geïnvesteerd hebt in het meedenken over de opzet van mijn proefschrift en in het geven van constructieve feedback.

De leden van de beoordelingscommissie: Prof. dr. E.A.L. Biessen, Prof. dr. F.C.S. Ramaekers, Prof. dr. J.J. Voorberg en Prof. dr. G. Vriend, wil ik hartelijk bedanken voor de tijd die zij hebben besteed aan het lezen en beoordelen van mijn proefschrift.

Tijdens mijn PhD-periode heb ik de kans gehad om samen te werken met verschillende mensen van afdelingen in het azM en universiteiten in binnen- en buitenland. Prof. dr. Lutgens, beste Esther, van jou heb ik een hoop geleerd over atherosclerose en het 
immuunsysteem. Zowel op wetenschappelijk als persoonlijk vlak heb ik veel gehad aan jouw adviezen. Jouw kennis was onmisbaar voor het opzetten van mijn muisexperiment naar het effect van APC op atherosclerose. Voor alle hulp bij de muisexperimenten, FACS analyse en kleuring/analyse van coupes wil ik Linda, Tom, David, Jeroen, Erwin, Jack, Anique en Judith van de afdeling Vasculaire Pathologie van het azM in Maastricht van harte bedanken. Daarnaast wil ik Prof. dr. van der Poll, Prof. dr. Schultz, Dr. Horn en Maryse van het AMC in Amsterdam bedanken voor het verzamelen en beschikbaar stellen van plasma samples van sepsis patiënten en voor hulp bij het analyseren van data en het schrijven van het manuscript. I would like to thank Dr. Villoutreix and Dr. Sperandio from the University of Paris Diderot for their contribution to the in silico virtual ligand screening of small molecule inhibitors of activated protein C. For their contribution to chapter 7 of my thesis I would like to thank Dr. García de Frutos and Dr. Aresté from the Institute of Biomedical Research of Barcelona, Prof. dr. Söhnlein (AMC Amsterdam and Ludwig-Maximilians-University Munich) and Dr. OrtegaGómez (Ludwig-Maximilians-University Munich).

Prof. dr. ten Cate, beste Hugo, ik wil jou en Sarah bedanken voor de tijd die jullie gestoken hebben in ons gezamelijke onderzoek naar het effect van APC varianten op ischemie/reperfusie schade en voor jullie bijdrage aan mijn review artikel. Prof. dr. Hemker, bedankt voor het goede idee om een niet-antistollende heparine variant te gebruiken in ons onderzoek en voor de hulp bij het schrijven van het manuscript en de analyse van muizen trombine generatie data. Dr. Wagenvoord, bedankt voor de hulp bij de productie van AADH. Niko, bedankt voor de lange dagen die je gemaakt hebt om neutrofielen te isoleren, deze te stimuleren tot NET vorming en deze NETs te visualiseren met elektronen- en fluorescentie miscroscopie. Deze experimenten waren cruciaal voor de acceptatie van mijn manuscript.

Ik wil al mijn directe collega's bedanken voor alle gezellige momenten en hulp in de afgelopen jaren. Dr. Du, I enjoyed being your roommate and your friend. You were always very friendly and I could always talk to you about what I had on my mind. It was always fun to play Mahjong and badminton together with you and other friends. It's a pity that you will be back in China at the $25^{\text {th }}$ of November, but I am sure we will meet again in the future.

Mijn 2 paranimfen: Farida en Francesca, wil ik ook graag bedanken. Zowel op het werk als daarbuiten heb ik veel goede gesprekken met jullie gehad. Jullie waren altijd geïnteresseerd in hoe het met me ging, ook nadat ik naar Mannheim verhuisd was. Ik heb goede herinneringen aan de wandelingen die we samen gemaakt hebben. Francesca, jij was het laatste jaar mijn vaste badmintonpartner op donderdagavond en liet me kennis maken met de beste Italiaanse restaurants in Maastricht. Bedankt voor de keren dat ik je 
laptop mocht lenen voor sollicitatiegesprekken via Skype. Farida, jij hield net als ik van lekker eten en drinken. Ik vond het altijd gezellig met jou op borrels en feestjes en heb goede herinneringen aan onze vakantie in India.

Roy, heel erg bedankt voor alle APC varianten die je voor mij gemaakt hebt. In het lab was je van onschatbare waarde. Er waren geen vragen die je niet kon beantwoorden, geen problemen die je niet kon verhelpen en dankzij jou was het lab altijd ordelijk en schoon. Ik heb het altijd gezellig gevonden om jou als kamergenoot te hebben. Je was altijd in voor een praatje en voor een biertje op vrijdagmiddag. Barbara, je was altijd gedreven, hardwerkend en erg ambitieus. Dankzij jou heb ik zeker ook wat van die instelling overgenomen. Je was een goede reisgids en ik vond het erg leuk dat je mij en andere colleaga's kennis hebt laten maken met je moederland Polen. Bedankt voor je gastvrijheid toen ik een slaapplaats nodig had met Jiang's promotie. Kanin, thank you for the time you spent on making models to study the interaction of wt- and 5A-APC with histones and for the discussions we had about citrullination of histones and other scientific topics.

Simone, bedankt voor alle hulp die je me gegeven hebt in het lab en voor alle kennis die je overgedragen hebt. Ik weet nog goed dat we samen vele uren doorgebracht hebben bij pathologie voor het fotograferen, kleuren en analyseren van coupes. Stella, bij jou kon ik altijd terecht met vragen over trombine-generatie, kinetische assays en allerlei andere lab-gerelateerde dingen. Je was altijd bereid me te helpen en advies te geven. Bedankt daarvoor. Je was erg belangrijk voor de sfeer en gezelligheid binnen de afdeling en stimuleerde mensen deel te nemen aan activiteiten zoals de Kerstmannenloop, Duckrace en Singles Party op Valentijnsdag.

Ik wil graag de secretaresses Trees, Lidewij en Elsa bedanken voor al hun hulp bij het inschrijven voor congressen, regelen van treinkaartjes en bij allerlei zaken die geregeld moesten worden voor mijn promotie. Voor hun waardevolle wetenschappelijke bijdrage aan de werkbesprekingen op donderdagochtend wil ik graag Jan, Guido, Betta en Rory bedanken. Ik heb veel geleerd van jullie vragen, tips en opmerkingen. Deze zijn belangrijk geweest voor mijn wetenschappelijke vorming.

Al mijn andere directe collega's: Marie, Alexandra, Linda, Kim, Pieter, Stijn, Ingrid, Tanja, Dennis, Liesbeth, Hans, Kristien, Sameera, Connie, Olivier, Mahesh, Svetlana en overige collega's van de afdeling Biochemie en Synapse wil ik graag bedanken voor alle hulp, nuttige discussies en leuke momenten.

Tot slot wil ik graag mijn familie en vrienden bedanken voor hun steun, betrokkenheid en interesse de afgelopen jaren. Zonder jullie was dit proefschrift er nooit gekomen. 
Mama en papa, bedankt dat jullie altijd voor me klaar stonden, me altijd de vrijheid hebben gegeven om mijn eigen keuzes te maken en me altijd gesteund hebben. Judith, Tamas en Lorainn; Rick en Suzanne, bedankt dat ik altijd bij jullie terecht kon met mijn verhalen en voor de gezelligheid tijdens feestdagen. Marly, je was de afgelopen jaren mijn beste vriendin. We hebben samen veel leuke dingen meegemaakt en je hebt me altijd gesteund op de momenten dat ik het even niet meer zag zitten tijdens mijn promotietraject. Ik ben blij dat we nog steeds goed contact hebben sinds ik in Mannheim woon en hoop dat we nog lang vriendinnen zullen blijven. Tot slot wil ik alle dames van mijn voetbalteam bedanken. Hoewel ik al een jaar geleden gestopt ben, was voetbal een belangrijke uitlaatklep tijdens het grootste deel van mijn promotieperiode en kijk ik met veel plezier terug op alle mooie momenten die we samen meegemaakt hebben. 

Curriculum Vitae

List of publications 


\section{Curriculum Vitae}

Karin Wildhagen was born on February $3^{\text {rd }} 1985$ in Goirle, The Netherlands. She completed her secondary education at the Mill-Hill College in Goirle in 2003. Afterwards she started with a bachelor General Health Sciences at the Maastricht University. She succesfully participated in the Honours Programme and obtained her Bachelor degree with Distinction in 2007. In the same year she started with the research master Cardiovascular Biology and Medicine, organized by the Cardiovascular Research Institute Maastricht (CARIM), at the Maastricht University. During her master study she performed her junior internship at the department of Immunology and her senior internship at the department of Biochemistry. Both departments were part of CARIM at Maastricht University. In 2009, after obtaining her master degree, she started her $\mathrm{PhD}$ research at the department of Biochemistry (CARIM, Maastricht University) under supervision of Dr. G.A.F. Nicolaes, Prof. dr. T.M. Hackeng and Prof. dr. C.P.M. Reutelingsperger. During her $\mathrm{PhD}$ project, she has studied the cytoprotective functions of the anticoagulant biomolecules activated protein $\mathrm{C}$ (APC) and heparin in murine disease models of sepsis, atherosclerosis and myocardial ischemia/reperfusion injury. She presented her research at congresses of the International Society on Thrombosis and Haemostasis (ISTH) in Amsterdam and of the Dutch Association for Thrombosis and Haemostasis (NVTH) in Koudekerke. From June 15th 2014 onwards she is working as Application Specialist at the Customer Support Center of Roche Diagnostics in Mannheim, Germany. 


\section{List of publications}

\section{Papers}

- Nicolaes GAF, Bock PE, Segers K, Wildhagen KCAA, Dahlbäck B, Rosing J. Inhibition of thrombin formation by active site mutated (S360A) activated protein C. J Biol Chem. 2010;285(30):22890-900.

- Wildhagen KCAA, Lutgens E, Loubele STGB, ten Cate H, Nicolaes GAF. The structure-function relationship of activated protein C. Lessons from natural and engineered mutations. Thromb Haemost. 2011;106(6):1034-45.

- Wildhagen KCAA*, García de Frutos P*, Reutelingsperger CPM, Schrijver R, Aresté C, OrtegaGómez A, Deckers NM, Hemker HC, Soehnlein O, Nicolaes GAF. Non-anticoagulant heparin prevents histone-mediated cytotoxicity in vitro and improves survival in sepsis. Blood 2014;123(7):1098101.

- Sperandio O*, Wildhagen KCAA*, Schrijver R, Wielders S, Villoutreix BO, Nicolaes GAF. Identification of novel small molecule inhibitors of activated protein C. Thromb Res 2014;133(6):110514.

- Wildhagen KCAA, Schrijver R, Beckers L, ten Cate H, Reutelingsperger CPM, Lutgens E, Nicolaes GAF. Effects of exogenous recombinant APC in mouse models of ischemia reperfusion injury and of atherosclerose. PLoS One 2014;9(7):e101446.

- Wildhagen KCAA, Du J, Schrijver R, Reutelingsperger CPM, Nicolaes GAF. Proteolysis of extracellular histones by activated protein C. In preparation.

- Wildhagen KCAA, Wiewel M, Schultz MJ, Horn J, Reutelingsperger CPM, van der Poll T, Nicolaes GAF. Extracellular histone $\mathrm{H} 3$ levels are associated with sepsis-induced organ failure and serve as predictive biomarkers of mortality in ICU sepsis patients. In preparation.

* These authors contributed equally to this work 


\section{Oral presentations}

- Wildhagen KCAA, García de Frutos P, Reutelingsperger CPM, Schrijver R, Aresté C, Ortega-Gómez A, Deckers NM, Hemker HC, Soehnlein O, Nicolaes GAF. Protection against sepsis and ConAinduced acute hepatitis by a non-anticoagulant heparin analogue. Symposium Nederlandse Vereniging voor Trombose en Hemostate (NVTH), Koudekerke, The Netherlands, 2013.

- Wildhagen KCAA, Sperandio O, Schrijver R, Wielders S, Villoutreix BO, Nicolaes GAF. Discovery and characterisation of small molecule inhibitors of human activated protein C. Symposium Nederlandse Vereniging voor Trombose en Hemostate (NVTH), Koudekerke, The Netherlands, 2014.

\section{Poster presentations}

- Wildhagen KCAA, García de Frutos P, Reutelingsperger CPM, Schrijver R, Aresté C, Ortega-Gómez A, Deckers NM, Hemker HC, Soehnlein O, Nicolaes GAF. Non-anticoagulant heparin analogue protects against ConA-induced sterile inflammation and sepsis. XXIV Congress of the International Society on Thrombosis and Haemostasis (ISTH), Amsterdam, The Netherlands, 2013.

- Wildhagen KCAA, van Bilsen M, Lutgens E, ten Cate H, Nicolaes GAF. Unraveling the cytoprotective effects of activated protein C in ischemic injury. PhD-training Course Vascular Biology, Dutch Heart Foundation, Arnhem, The Netherlands 2010. 